\title{
AN EXPERIMENTAL INVESTIGATION OF A GOLDSCHMIED PROPULSOR
}

\author{
A Thesis \\ presented to \\ the Faculty of California Polytechnic State University \\ San Luis Obispo \\ In Partial Fulfillment \\ Of the Requirements for the Degree \\ Master of Science in Aerospace Engineering
}

By

Joshua Roepke

August 2012 


\section{Copyright $\odot 2012$}

By

Joshua Roepke

ALL RIGHTS RESERVED 


\section{COMMITTEE MEMBERSHIP}

TITLE

AUTHOR

DATE SUBMITTED

COMMITTEE CHAIR

COMMITTEE MEMBER

COMMITTEE MEMBER

COMMITTEE MEMBER
An Experimental Investigation of a Goldschmied Propulsor

Joshua Roepke

August, 2012

Dr. Tina Jameson

Assistant Professor, Aerospace Engineering Department

Dr. Russell Westphal

Professor, Mechanical Engineering Department

Dr. Rob McDonald

Associate Professor, Aerospace Engineering Department

Dr. David Marshall

Associate Professor, Aerospace Engineering Department 


\author{
ABSTRACT \\ An Experimental Investigation of a Goldshmied Propulsor \\ Joshua Roepke
}

A wind tunnel investigation of an axisymmetric bluff body, known as a Goldschmied propulsor, was completed. This model conceptually combines boundary layer control and boundary layer ingestion into a single complementary system that is intended to use energy to reduce the axial force on the body by eliminating separation and increasing the pressure recovery aft of the body's maximum thickness. The goal of the current project was to design, fabricate, and fully document the performance of a wind tunnel model incorporating the Goldschmied propulsor concept and complete an examination of its aerodynamic performance. The investigation took place at California Polytechnic State University, San Luis Obispo in the Aerospace Engineering Department's subsonic $3 \mathrm{ft}$ by $4 \mathrm{ft}$ wind tunnel. The model is 38.5 inches in length and 13.5 inches in diameter with a discrete suction slot at $85 \%$ of the body length and an embedded propulsor that provides the suction flow, expelling it out of the model's aft end. The experiment included measurements of surface pressure, total axial force, suction mass flow rate, fan thrust, fan torque, fan speed, and input fan power.

The size of the suction slot and amount of input fan power were the main test variables in the 54 data point test matrix that was completed at a length Reynolds number of 1.34 million and a tunnel speed of $66 \mathrm{ft} / \mathrm{s}(20 \mathrm{~m} / \mathrm{s})$. The model was able to achieve fully attached flow on the aftbody with as little as $100 \mathrm{~W}$ of input power and a net positive (forward) axial force coefficient of 0.12 with as little as $200 \mathrm{~W}$ of input power. The model was also able to achieve a peak axial pressure force coefficient of 0.005 in the forward direction with an input power of $500 \mathrm{~W}$ and a slot gap of $1.6 \%$ of the body length. A slightly lower axial pressure force coefficient of 0.0045 was achieved with only $200 \mathrm{~W}$ of input power and a slot gap of $0.7 \%$ of the body length. The peak axial pressure force for most tested slot gaps occurred at about 200W of input power, and a slot gap of $0.7 \%$ of the body length resulted in the best overall performance for most input power settings. Two different suction slot configurations, a simple gap and a cusp, were tested, and no significant performance differences were seen between them. The pressure coefficient data showed similar trends as test data from 1956 of a similar model at higher Reynolds number, but it did not show complete agreement. Despite these positive aspects of the investigation, a simple power based comparison between the collected data and a conventional non-integrated propulsor does not show a performance improvement for the Goldschmied propulsor. 


\section{DEDICATION}

This thesis is dedicated to 2 people that have really been immense influences on me, and who I miss daily. Without both, I would not be the person I am today. First, this is dedicated to my best friend and brother, Aaron Roepke whose unbridled enthusiasm and love of life still inspires me after these many challenging years without him. Secondly, my grandfather, Eldon Roepke, whose simple brilliance, unparalleled work ethic, and, above all, biblical strength are things I strive to replicate in my life. 


\section{ACKNOWLEDGEMENTS}

I would like to acknowledge and thank so many people that went beyond their normal duties to help with this project.

I would like to thank Mark Moore, Bill Fredericks, and Alaa Emiliqui from NASA Langley Research Center for the support and funding for this project.

I would like to sincerely thank Dr. Tina Jameson for taking me on as a graduate student, even though her expertise is not in what I wanted to pursue. I am sad to see her leave Cal Poly and am proud to say she was my adviser. I wish her good luck in her future endeavors.

I would like to thank Greg Altmann for his countless hours of work in the tunnel, literally countless. Without Greg's hard work, the Cal Poly Aero Wind Tunnel would not be what it is today and everyone would still have "issues" with their data. More importantly, I would like to specifically thank Greg for challenging me throughout the process of renewing the wind tunnel. I think I learned more in those few months with Greg than I did in the rest of my graduate school experience.

I would like to thank Nicole Thomason, who worked on this project along side me. I would like to thank her for her hard work and strong commitment to the project, and for always reminding me of the bigger picture. She was constantly keeping me from being bogged down in things that were not very important. I do not know where the project would have gone without her.

I would like to thank Dr. Rob McDonald for always doing whatever he could within his power to help me. Whether it was design advice, supplies from other resources on campus, or coming in on the weekend to help with calibrations and run the tests, he was an immense help.

I would like to give a very large thank-you to Dr. Russell Westphal for always being willing to share his expert advice, lending a voice of reason to my project, and letting me borrow his really cool equipment. It was very refreshing to have someone that would tell me based on real world experience that what I wanted to do would not work quite as I planned or that it was more difficult than I expect, and, most importantly, give me the reasons why. His advice shaped both the wind tunnel inlet redesign and many aspects of the project at hand. 
I would also like to thank those that helped and contributed in their own way in one form or another. Joel Belben was a great partner when I needed to reverse engineer something in the lab. Cody Thompson helped manufacture some of the model pieces and gave me access to his tools. Dr. Jin Tso always reminded me how "so simple" everything is. John Gorski helped machine some parts of the model. The FSAE club on campus let me take up space in their room in the hangar and use their composite supplies. Dr. Marshall helped me to realize that singlehandedly doing the wind tunnel test and CFD was a bad idea. These small contributions really meant a lot and I would not have been able to get this project done without them.

Last, yet most important, I would like to thank my wife, Veronica, for putting up with the long hours at my desk, weekends away at school, and my general sleep deprived demeanor over the course of this long project. 


\section{TABLE OF CONTENTS}

PAGE

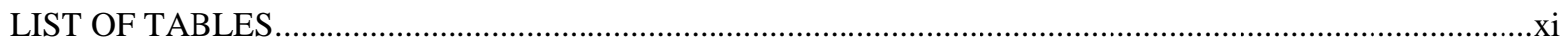

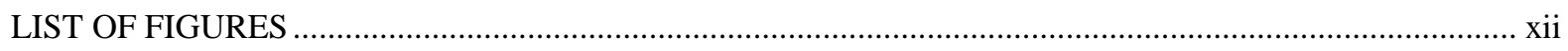

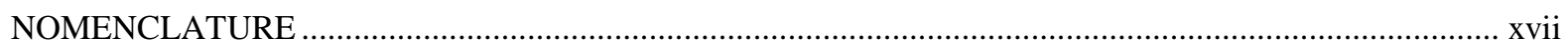

\section{CHAPTER}

$1 \quad$ INTRODUCTION

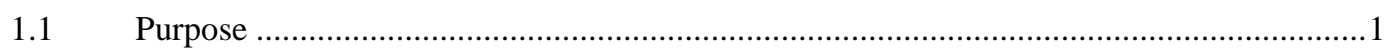

2 BACKGROUND

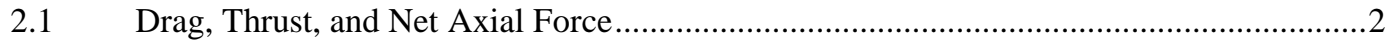

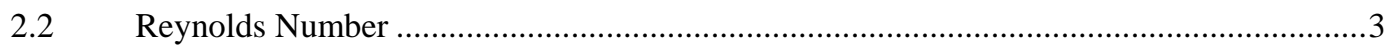

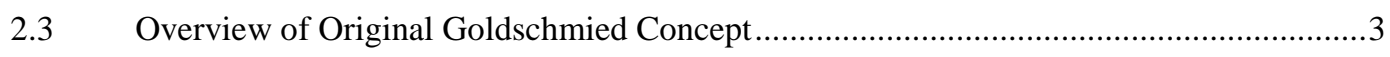

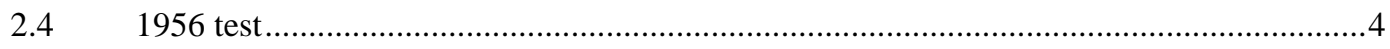

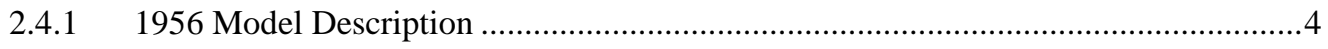

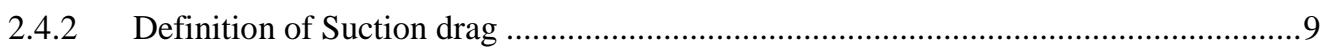

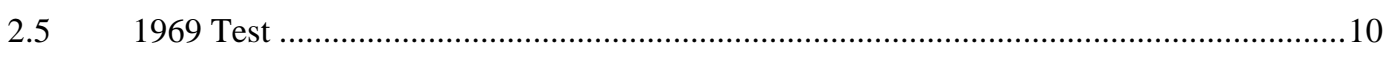

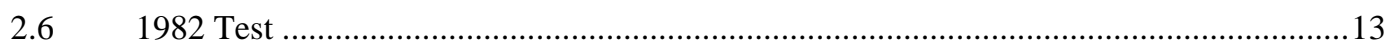

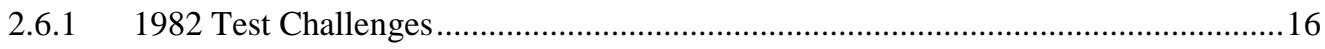

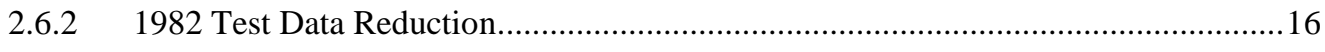

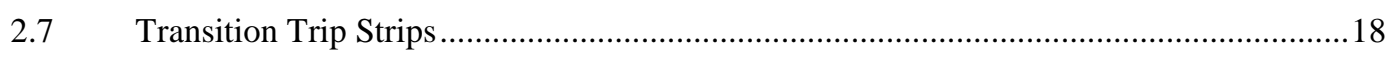

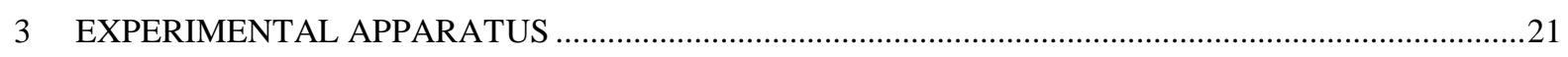

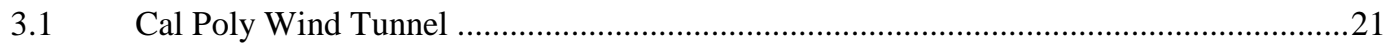

3.1.1 Cal Poly Wind Tunnel History ……................................................................2

3.1.2 Cal Poly Wind Tunnel Recent Modifications ........................................................2

3.2 New Goldschmied Propulsor Model ………............................................................... 24

viii 


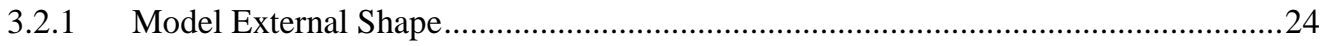

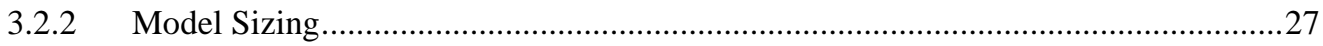

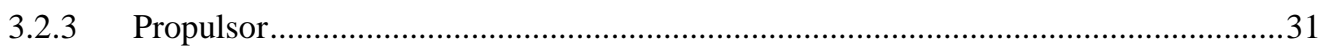

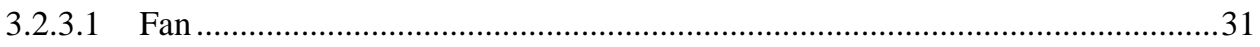

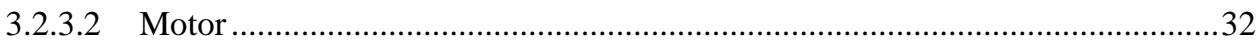

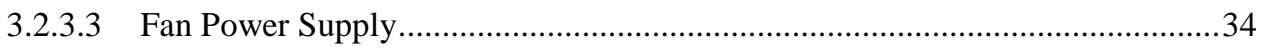

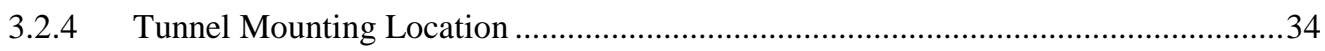

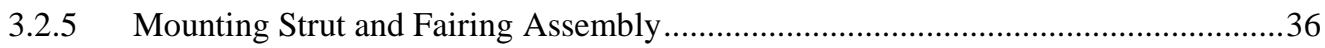

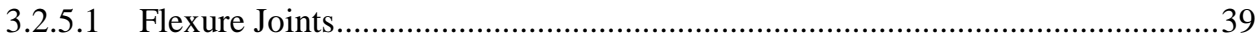

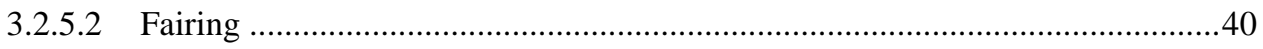

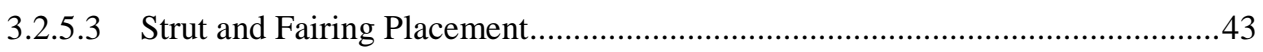

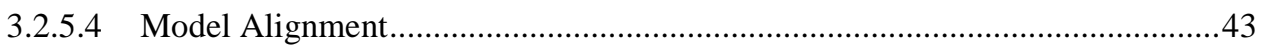

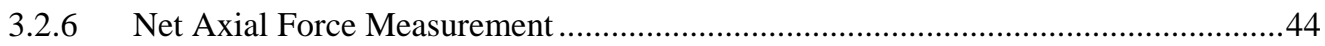

3.2.6.1 Load Cell Characteristics...............................................................................

3.2.6.2 Axial Force Free Body Diagram....................................................................... 45

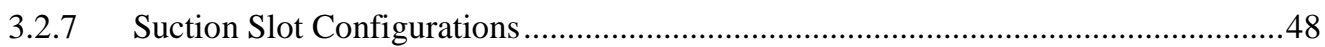

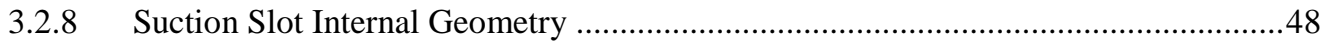

3.2.9 Suction Mass Flow Rate Measurement.................................................................

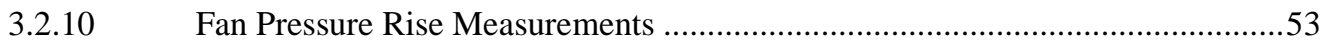

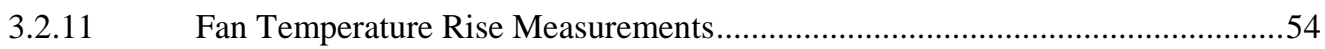

3.2.12 Fan Thrust \& Fan Torque Measurements ………….........................................55

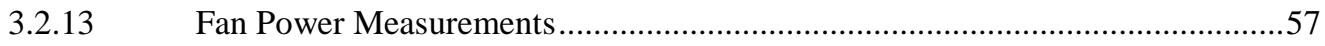

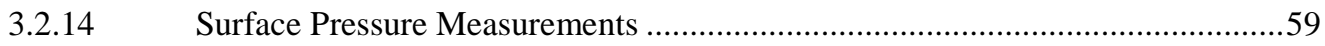

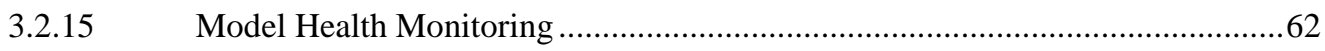

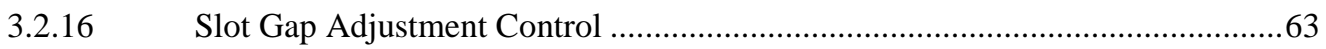

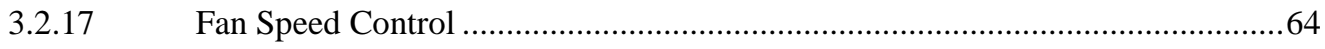

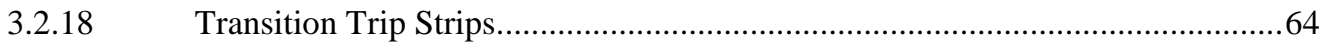

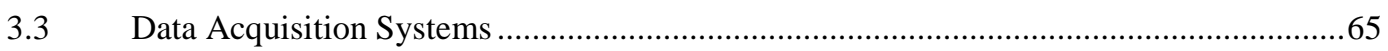




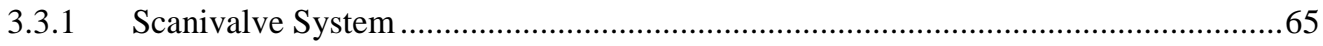

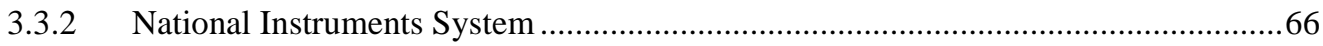

3.3.3 Measurement Computing System ……….............................................................67

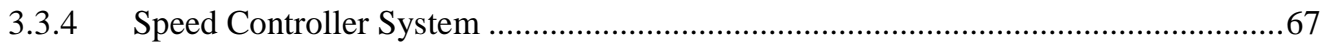

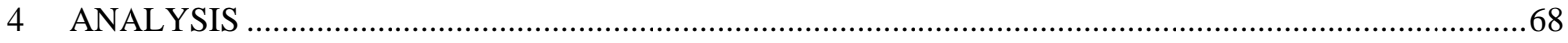

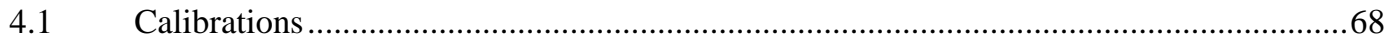

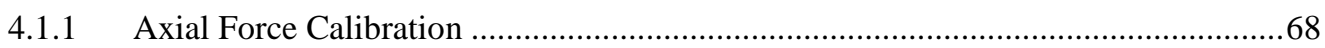

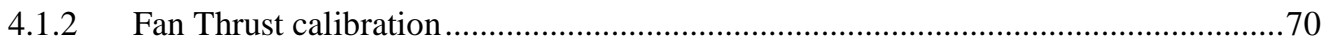

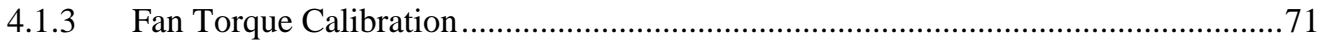

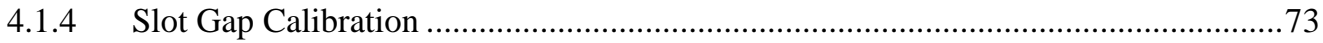

4.1.5 Power Supply Voltage and Current Calibration .........................................................

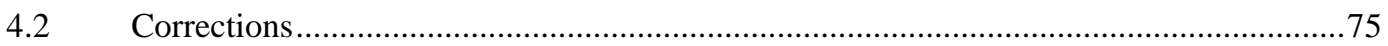

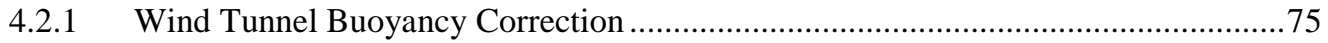

4.2.2 Wind Tunnel Static, Dynamic, \& Total Pressure Corrections ....................................77

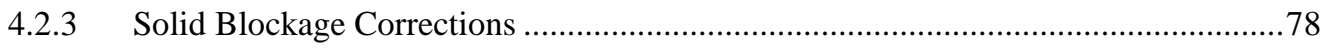

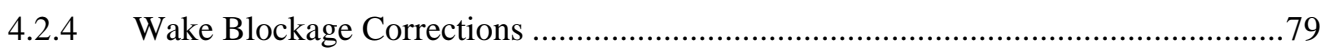

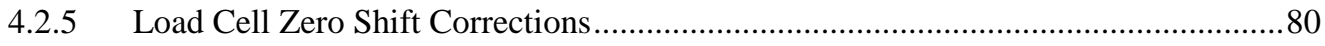

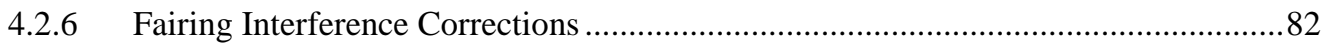

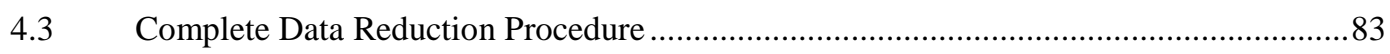

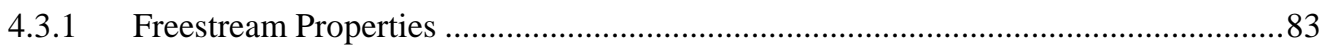

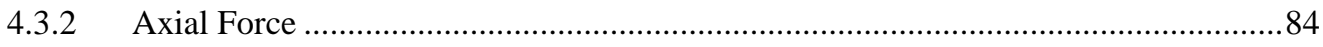

4.3.3 Pressure Force

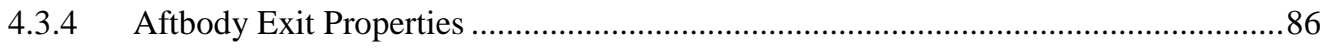

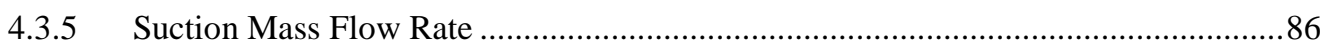

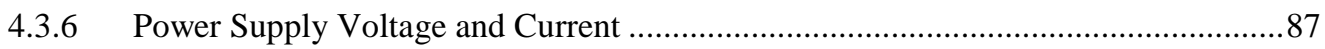

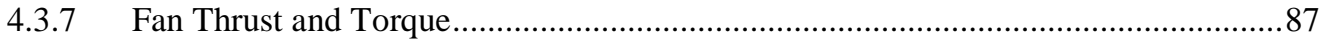

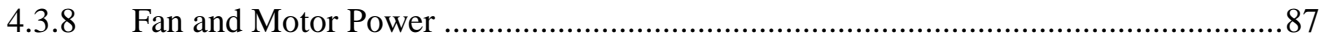

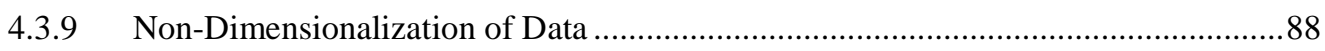




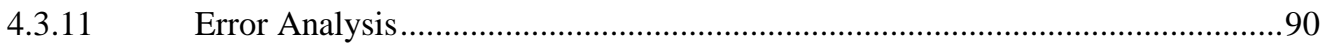

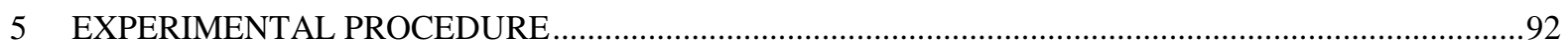

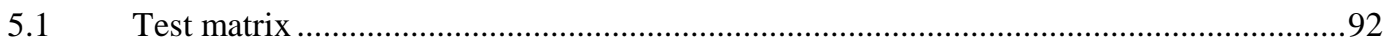

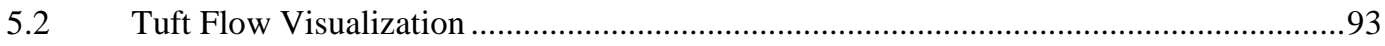

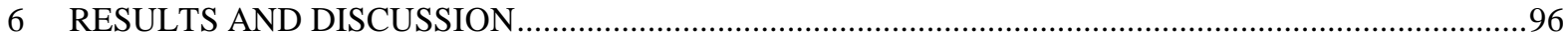

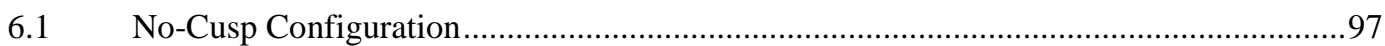

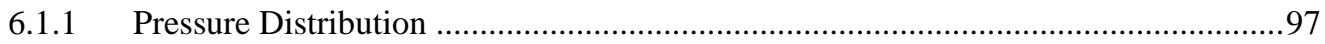

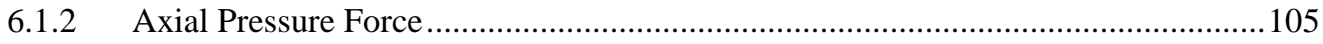

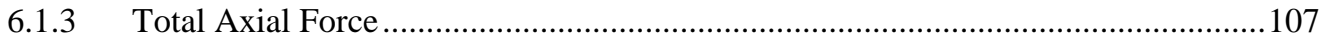

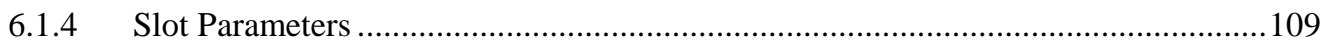

6.2 Comparison Between No Cusp and Cusp A ………......................................................110

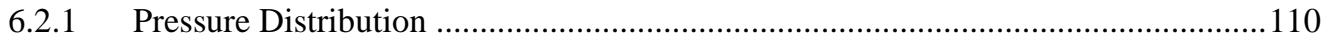

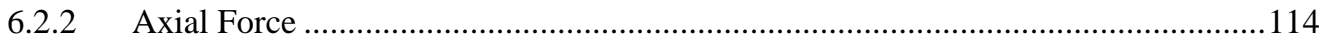

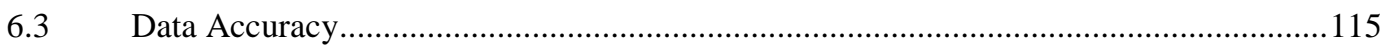

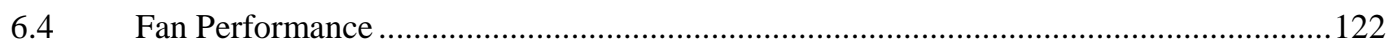

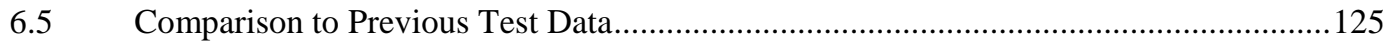

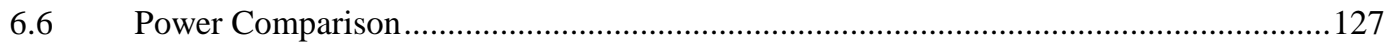

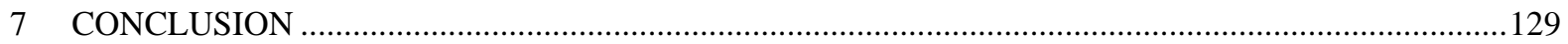

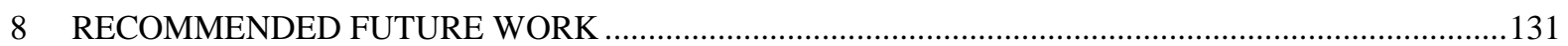

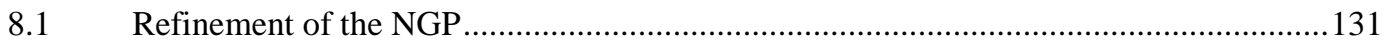

8.2 Test Matrix \& Data Aquisition System.......................................................................... 132

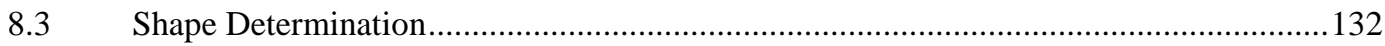

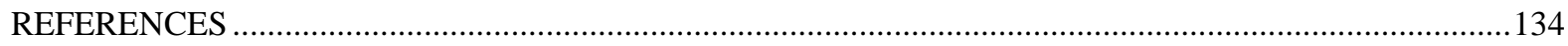

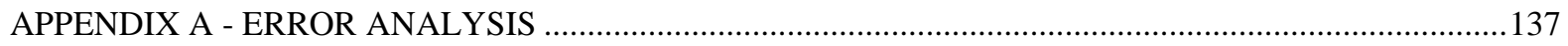

APPENDIX B - PROCESSED DATA WITH ERROR ESTIMATES ..........................................................139

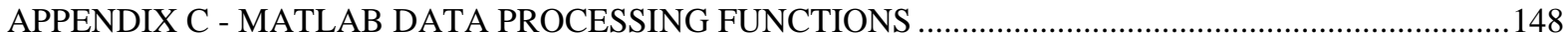

APPENDIX D - DERIVATION OF THE AXIAL PRESSURE FORCE EQUATION ....................................173 
APPENDIX E - SURFACE POINTS AND PRESSURE PORT LOCATIONS ....

APPENDIX F - SCANIVALVE INFORMATION \& TROUBLESHOOTING ........................................179

APPENDIX G - PRESSURE MEASUREMENT FITTINGS, TUBING \& GENERAL ADVICE ..................180

APPENDIX H - CAL POLY WIND TUNNEL VFD OPERATION ..................................................... 


\section{LIST OF TABLES}

Table 3-1. Summary of the geometric parameters of the NGP and previous Goldschmied models.....................30

Table 3-2. Summary of the motor properties for the two motors used in testing of the NGP. ............................33

Table 4-1. Summary of static, dynamic, and total pressure correction factors that were used for all analyses. ...78

Table 5-1. Extra data points that were taken to evaluate the repeatability of the data......................................93

Table 6-1. Table describing the notation used for the different test parameters...............................................96

Table 6-2. Summary of select test parameters and their corresponding error estimates....................................116 


\section{LIST OF FIGURES}

Figure 2-1. General configuration of the model used in the 1956 test of a Goldschmied propulsor. ${ }^{4}$ 6

Figure 2-2. One of the aftbody modifications to the Lighthill 34\% thick airfoil which resulted from the use of the electrostatic tank. ${ }^{4}$

Figure 2-3. A schematic of the three aft body configurations tested during the 1956 test of a Goldschmied propulsor. ${ }^{4}$.

Figure 2-4. The only available two data points from the 1969 test of the Goldschmied propulsor compared against data from the 1956 test. $^{8}$

Figure 2-5. Depiction of a suction slot airfoil that uses a trapped vortex to help the suction flow to smoothly enter the suction slot. ${ }^{9}$

Figure 2-6. Fabio R. Goldschmied and the SAP model with the short aftbody as used in the 1982 wind tunnel test. ${ }^{5}$

Figure 2-7. Fabio R. Goldschmied and the SAP model with the long aftbody and cruciform tail as used in the 1982 wind tunnel test. ${ }^{5}$

Figure 2-8. Schematic of the SAP concept that shows the locations of the boundary layer rake, the fan \& flow straightener devices, as well as the general dimensions of the body. ${ }^{5}$

Figure 2-9. Schematic of the long aftbody with the tail and associated dimensions. ${ }^{5}$

Figure 3-1. Layout of the Cal Poly Aerospace Engineering 3'x4' indraft wind tunnel.

Figure 3-2. The aluminum and Plexiglas test section and accompanying traverse that was used in the current testing.

Figure 3-3. Comparison of surface profiles from various sources. All data is non-dimensionalized by the body length..$^{5,8,4,15}$

Figure 3-4. Comparison of same surface profiles from Fig. 3-3. All data is non-dimensionalized by the length of the forbody. ${ }^{5,8,4,15}$

Figure 3-5. Comparison of the smoothed (grey) and unsmoothed (black) model surfaces.

Figure 3-6. Comparison of the bounradry layer shape factor for the smoothed and unsmoothed surfaces.

Figure 3-7. The percent increase in the freestream speed seen by the model as a function of the model size compared to the wind tunnel test section size.

Figure 3-8. General profile layout and dimensions of the NGP.

Figure 3-9. The fan performance curve (curve \#2) for a fan considered for use in the NGP. This plot is for a model $4114 \mathrm{~N} / 2 \mathrm{H} 8 \mathrm{P}$ fan from Ebmpapst Inc. ${ }^{16}$ 31 
Figure 3-10. Schubeler DS-30-DIA HDT fan and motor (black carbon fiber) preliminarily installed in the aluminum aftbody structure during fabrication.

Figure 3-11. Data supplied by the fan manufacturer for the fan and 700W motor together. ${ }^{17}$

Figure 3-12. Voltage \& Current characteristics of the HP6012B power supply used to power the EDF during all testing of the NGC. ${ }^{18}$

Figure 3-13. General configuration of the model and model mount assembly.

Figure 3-14. Underneither view of the model mounting base, pivots, flexures, and strut. The left picture is without the load cell and load cell mount, while the right view has them included. .38

Figure 3-15. Actual model mount with the load cell for comparison with the CAD model.

Figure 3-16. Flexure joints were used as the pivots because they allow frictionless rotation.

Figure 3-17. The fairing and baseplate mounted in the tunnel with the strut, stainless steel pressure tubes and wiring visible.

Figure 3-18. Bottom mounting block showing the adjustment set screws and shims used for alignment in the pitch and yaw axis.

Figure 3-19. Layout diagram (left) and free body diagram (right) of the model strut.

Figure 3-20. Cross section of the cusp used for the second half of the test matrix. The height of this section is approximately 0.4 " tall.

Figure 3-21. Diagram of the flow path of the suction flow. .50

Figure 3-22. Initial suction mass flow rate calibration curves. .52

Figure 3-23. Picture of the fan upstream pressure ports and suction flow thermocouple.

Figure 3-24. Picture of the suction flow thermocouple just upstream of the fan.

Figure 3-25. CAD model of the load cells mounted in the aftbody of the model. The horizontally mounted load cells measure fan torque, and the vertically mounted load cells measure fan thrust.

Figure 3-26. Actual installation of the load cells in the aftbody with the wiring and tubing routed around them.

Figure 3-27. Close up views of the load cells installed.

Figure 3-28. Surface of the NGP showing the surface pressure ports used to calculate the axial pressure force on the model.

Figure 3-29. Designation of the surface pressure port locations (Top) and their relative influence on the overall pressure force (Bottom). The circled ports ended up being used for other measurements during testing.

Figure 4-1. Apparatus used to calibrate the axial force load cell.

Figure 4-2. Calibration curve for axial force. 
Figure 4-3. Apparatus used to calibrate the fan thrust load cells.

Figure 4-4. Calibration curve for the fan thrust load cells used for all data reduction.

Figure 4-5. The apparatus used to calibrate the torque load cells.

Figure 4-6. Calibration curve of the fan torque used for all data reduction.

Figure 4-7. Calibration curve of slot gap that was used for all data without a cusp.

Figure 4-8. Power supply current calibration curve used fror all data.

Figure 4-9. Wind tunnel axial variation of static pressure, total pressure, and dynamic pressure. The dashed lines are estimates based on an assumption of a constant pressure gradient after the last measured point.

Figure 4-10. Diagram of the zero shift of the load cells.

Figure 4-11. The silver dummy strut was used to measure the influence of the fairing on the measured axial force of the model.

Figure 4-12. Diagram of a generic surface pressure port configuration used to derive the axisymmetric pressure drag equation.

Figure 4-13. Subcritical (solid line) and supercritical (dashed line) surface pressure data of a sphere used to validate the equation derived to calculate axial pressure force. ${ }^{22}$.......

Figure 5-1. Test matrix for the wind tunnel test of the NGP. The smallest slot gap with the cusp was not run, resulting in 54 total test points.

Figure 5-2. Tuft flow visualization showing fully attached flow on the aftbody

Figure 5-3. Tuft flow visualization showing fully separated flow on the aftbody.....

Figure 6-1. $\mathrm{C}_{\mathrm{p}}$ distribution for the case with no cusp and a slot gap of $0.4 \%$ of the body length.

Figure 6-2. $\mathrm{C}_{\mathrm{p}}$ distribution for the case with no cusp and a slot gap of $0.7 \%$ of the body length.

Figure 6-3. $\mathrm{C}_{\mathrm{p}}$ distribution for the case with no cusp and a slot gap of $1.0 \%$ of the body length. .99

Figure 6-4. $\mathrm{C}_{\mathrm{p}}$ distribution for the case with no cusp and a slot gap of $1.3 \%$ of the body length. 100

Figure 6-5. $\mathrm{C}_{\mathrm{p}}$ distribution for the case with no cusp and a slot gap of $1.6 \%$ of the body length.

Figure 6-6. $\mathrm{C}_{\mathrm{p}}$ distribution for the case with no cusp and a shaft power of $0 \mathrm{~W}$ and various slot gaps 102

Figure 6-7. $\mathrm{C}_{\mathrm{p}}$ distribution for the case with no cusp and a shaft power of $86 \mathrm{~W}$ and various slot gaps. 102

Figure 6-8. $\mathrm{C}_{\mathrm{p}}$ distribution for the case with no cusp and a shaft power of $171 \mathrm{~W}$ and various slot gaps.........103

Figure 6-9. $\mathrm{C}_{\mathrm{p}}$ distribution for the case with no cusp and a shaft power of $258 \mathrm{~W}$ and various slot gaps.........103

Figure 6-10. $\mathrm{C}_{\mathrm{p}}$ distribution for the case with no cusp and a shaft power of $343 \mathrm{~W}$ and various slot gaps....... 104

Figure 6-11. $\mathrm{C}_{\mathrm{p}}$ distribution for the case with no cusp and a shaft power of $427 \mathrm{~W}$ and various slot gaps. 104 
Figure 6-12. Axial pressure force coefficient for various slot gaps and fan shaft power settings.

Figure 6-13. Axial pressure force as a function of mass flow rate for various power settings.

Figure 6-14. Axial force coefficient of the no-cusp cases as a function of the slot gap for various fan power settings.

Figure 6-15. Axial force coefficient of the no-cusp cases as a function of the shaft power for various slot gaps.

Figure 6-16. Axial force coefficient of the no-cusp cases as a function of the suction flow coefficient for various slot gaps.

Figure 6-17. The slot pressure rise coefficient vs. suction flow coefficient for various shaft power settings. ...110

Figure 6-18. $\mathrm{C}_{\mathrm{p}}$ distribution for two slot gaps with and without a cusp and no suction.

Figure 6-19. $\mathrm{C}_{\mathrm{p}}$ distribution for two slot gaps with and without a cusp and $86 \mathrm{~W}$ of shaft power.

Figure 6-20. $\mathrm{C}_{\mathrm{p}}$ distribution for two slot gaps with and without a cusp and $171 \mathrm{~W}$ of shaft power.

Figure 6-21. $\mathrm{C}_{\mathrm{p}}$ distribution for two slot gaps with and without a cusp and $258 \mathrm{~W}$ of shaft power. 113

Figure 6-22. $\mathrm{C}_{\mathrm{p}}$ distribution for two slot gaps with and without a cusp and $343 \mathrm{~W}$ of shaft power. 113

Figure 6-23. $\mathrm{C}_{\mathrm{p}}$ distribution for two slot gaps with and without a cusp and $427 \mathrm{~W}$ of shaft power. 114

Figure 6-24. Comparison of the axial force coefficient for with and without a cusp at the suction slot.

Figure 6-25. $\mathrm{C}_{\mathrm{p}}$ distribution for the $\mathrm{NC} 2$ configuration with $343 \mathrm{~W}$ of shaft power comparing the original data and data taken later on in test matrix.

Figure 6-26. $\mathrm{C}_{\mathrm{p}}$ distribution for the $\mathrm{NC} 3$ configuration with $258 \mathrm{~W}$ and $427 \mathrm{~W}$ of shaft power comparing the original data and data taken later on in test matrix.

Figure 6-27. $\mathrm{C}_{\mathrm{p}}$ distribution for the NC3 configuration with $171 \mathrm{~W}, 343 \mathrm{~W}$, and $427 \mathrm{~W}$ of shaft power comparing the original data and data taken later on in test matrix.

Figure 6-28. $\mathrm{C}_{\mathrm{p}}$ distribution for the NC3 configuration with $86 \mathrm{~W}, 258 \mathrm{~W}$, and $427 \mathrm{~W}$ of shaft power comparing the original data and data taken later on in test matrix.

Figure 6-29. Total axial force as a function of slot gap for $427 \mathrm{~W}$ of shaft power comparing the orginal data and select repeated points to evaluate the precision of the measurements.

Figure 6-30. Total axial force as a function of slot gap for $343 \mathrm{~W}$ of shaft power comparing the orginal data and select repeated points to evaluate the precision of the measurements.

Figure 6-31. Total axial force as a function of slot gap for $258 \mathrm{~W}$ of shaft power comparing the orginal data and select repeated points to evaluate the precision of the measurements.

Figure 6-32. Total axial force as a function of slot gap for $171 \mathrm{~W}$ of shaft power comparing the orginal data and select repeated points to evaluate the precision of the measurements.

Figure 6-33. Total axial force as a function of slot gap for $86 \mathrm{~W}$ of shaft power comparing the orginal data and select repeated points to evaluate the precision of the measurements. 
Figure 6-34. Fan performance of the Hacker B40-11L and Schubeler DS30 fan installed in the NGP

Figure 6-35. Fan rotational speed vs. shaft power of the Hacker B40-11L and Schubeler DS30 fan installed in the NGP

Figure 6-36. Fan rotational speed vs. fan pressure rise for the Hacker B40-11L and Schubeler DS30 fan installed in the NGP

Figure 6-37. Fan rotational speed vs. fan thrust of the Hacker B40-11L and Schubeler DS30 fan installed in the NGP

Figure 6-38. The measured fan thrust, from the load cells, vs. the fan thrust calculated from the fan pressure rise and the fan swept area.

Figure 6-39. Comparison of $\mathrm{C}_{\mathrm{p}}$ distribution. GER-8399(4) is for $\mathrm{Re}_{\mathrm{L}}$ of $4.4 \times 10^{6}$ and $\mathrm{g} / \mathrm{L}$ of $1.2 \%$ and "medium suction." NGP data is for $\mathrm{g} / \mathrm{L}$ of $1.3 \%$ and $427 \mathrm{~W}$ shaft power and a $\mathrm{Re}_{\mathrm{L}}$ of $1.34 \times 10^{6}$.

Figure 6-40. Comparison of $\mathrm{C}_{\mathrm{p}}$ distribution. GER- $8399^{4}$ is for $\operatorname{Re}_{\mathrm{L}}$ of $7.1 \times 10^{6}$ and $\mathrm{g} / \mathrm{L}$ of $1.2 \%$ and no suction. NGP data is for $\mathrm{g} / \mathrm{L}$ of $1.3 \%$ and $0 \mathrm{~W}$ shaft power and $\mathrm{Re}_{\mathrm{L}}$ of $1.34 \times 10^{6}$.

Figure 6-41. Comparison between the axial force vs. shaft power relationship of a conventional propulsor an aircraft and for the NGP 


\section{NOMENCLATURE}

A

$\mathrm{A}_{\text {ref }}$

ADC

C

Cx

$\mathrm{C}_{\mathrm{A}}$

$\mathrm{C}_{\mathrm{A}, \mathrm{p}}$

$\mathrm{C}_{\text {Dex }}$

$\mathrm{C}_{\mathrm{p}}$

$\mathrm{C}_{\mathrm{p}, \mathrm{p}}$

$\mathrm{C}_{\mathrm{p}, \mathrm{s}}$

$\Delta C_{p, \text { slot }}$

$\mathrm{C}_{\mathrm{p}, \mathrm{t}}$

$\mathrm{C}_{\mathrm{q}}$

$\mathrm{CH}_{02}$

CTE

D

EDF

EMF

EMI

ESC

G

g

$\mathrm{H}$

$\mathrm{I}_{\mathrm{PS}}$

$\mathrm{F}$

$\mathrm{F}_{\mathrm{A}}$

$\mathrm{F}_{\mathrm{A}, \mathrm{p}}$

$\Delta \mathrm{F}_{\mathrm{A}, \mathrm{B}}$

Area

Reference Area, frontal area used all new test points

Analog to digital converter

Wind tunnel test section cross sectional area

This is a configuration designation for data taken with a cusp at slot gap ' $x$ ', see

Table 6-1 for further explanation

Axial force coefficient

$$
=\frac{F_{A}}{q_{\infty} A_{\text {ref }}}
$$

Axial pressure force coefficient

$$
=\frac{F_{A, p}}{q_{\infty} A_{\text {ref }}}
$$

External drag coefficient

$$
=\frac{p-P_{\infty}}{q_{\infty}}
$$

Propulsive power coefficient

Suction power coefficient

Change in the surface $C_{p}$ across the suction slot

Total power coefficient

Suction flow rate coefficient

$$
=\frac{\dot{m}_{s}}{\rho_{\infty} U_{\infty} A_{\text {ref }}}
$$

Total pressure loss coefficient between stations 0 and 2

Coefficient of thermal expansion

Drag, or diameter

Electric ducted fan

Electromotive force

Electromagnetic interference

Electronic speed controller

Unit of acceleration

Slot gap width

Boundary layer shape factor, $\delta^{*} / \theta$

Current exiting the power supply

Force

Net axial force on the model

Net axial pressure force on the model

Axial force increment due to buoyancy

xix 


\begin{tabular}{|c|c|c|}
\hline $\mathrm{L}$ & Model length & \\
\hline LE & Leading edge & \\
\hline $\mathrm{L}_{\mathrm{FB}}$ & Length of the forbody & \\
\hline$M_{A, f}$ & Moment of the fan about the rotation axis, (fan torque) & \\
\hline $\mathrm{MC}$ & Measurement Computing data acquisition system & \\
\hline$\dot{m}$ & Mass flow rate & \\
\hline $\mathrm{mV}$ & milivolts & \\
\hline $\mathrm{N}$ & Number of surface pressure ports & \\
\hline N/A & Not applicable or not available & \\
\hline $\mathrm{NCx}$ & $\begin{array}{l}\text { This is a configuration designation for data taken without a cusp at slot gap ' } \mathrm{x} \text { ', } \\
\text { see Table } 6 \text { - } 1 \text { for further explanation }\end{array}$ & \\
\hline NGP & New Goldschmied Propulsor, name for model of current test & \\
\hline NI & National Instruments data acquisition system & \\
\hline $\mathrm{P}_{\infty}$ & Freestream static pressure & \\
\hline $\mathrm{p}$ or $\mathrm{P}_{\mathrm{s}}$ & Static pressure & \\
\hline$P_{s, o s}$ & Static pressure at the outer slot & \\
\hline $\mathrm{P}_{\mathrm{s}, \mathrm{cs}}$ & Static pressure at the centerline of the slot & \\
\hline$P_{t}$ & Total pressure & \\
\hline$P_{t}$ & Total pressure & \\
\hline psf & Pounds per square foot & \\
\hline psi & Pounds per square inch & \\
\hline Q & Volume flow rate & \\
\hline$q_{\infty}$ & Freestream dynamic pressure & $=\frac{1}{2} \rho_{\infty} U_{\infty}^{2}$ \\
\hline $\mathrm{RC}$ & Radio Controlled & \\
\hline $\operatorname{Re}_{\mathrm{k}}$ & Roughness Reynolds number & $=\frac{\rho_{k} U_{k} k}{\mu_{k}}$ \\
\hline $\operatorname{Re}_{\mathrm{L}}$ & Reynolds number based on length & $=\frac{\rho_{\infty} U_{\infty} L}{\mu_{\infty}}$ \\
\hline$r_{i}$ & Radial location of the $\mathrm{i}^{\text {th }}$ surface pressure port & \\
\hline RMS & Root mean square & \\
\hline RPM & Revolutions per minute & \\
\hline$S$ & Model cross sectional area & \\
\hline $\mathrm{T}$ & Temperature or Thrust & \\
\hline $\mathrm{T}_{\mathrm{av}}$ & Thrust available & \\
\hline$U_{\infty}$ & Freestream speed & \\
\hline$U_{e}$ & Exit speed of a general propulsor & \\
\hline $\bar{u}_{e}$ or $\bar{U}_{e}$ & Average flow speed at the aftbody exit & \\
\hline
\end{tabular}


Introduction

\begin{tabular}{|c|c|}
\hline$u_{e}$ & Flow speed at the exit to the aftbody \\
\hline$u_{j}$ & Jet speed downstream of the exit to the aftbody \\
\hline $\mathrm{V}$ & Voltage \\
\hline $\mathrm{V}_{\mathrm{B}}$ & Volume of the model body \\
\hline $\mathrm{V}_{\mathrm{CZ}}$ & Zero force calibration voltage \\
\hline Vdc & DC voltage \\
\hline VFD & Variable frequency drive \\
\hline $\mathrm{V}_{\mathrm{PS}}$ & Voltage exiting the power supply \\
\hline$\Delta \mathrm{V}_{\mathrm{ZS}}$ & Voltage increment due to zero shift \\
\hline $\mathrm{W}$ & Watts \\
\hline $\mathrm{W}_{\mathrm{s}}$ & Theoretical work used by the suction system \\
\hline WOZ & Wind off zero \\
\hline $\mathrm{x}$ & Axial distance from the leading edge of the model \\
\hline \multicolumn{2}{|l|}{$\underline{\text { Greek }}$} \\
\hline$\delta^{*}$ & Boundary layer displacement thickness \\
\hline$\varepsilon_{S B}$ & Freestream speed increment due to solid blockage \\
\hline$\varepsilon_{W B}$ & Freestream speed increment due to wake blockage \\
\hline$\theta$ & Boundary layer momentum thickness \\
\hline$\rho_{\infty}$ & Freestream density \\
\hline$\mu V$ & Microvolt \\
\hline$\mu_{\infty}$ & Freestream kinematic viscosity \\
\hline
\end{tabular}

\section{Subscripts}

0

A or a

$\mathrm{ab}$

$\mathrm{f}$

c

CG

e

S

t

u

W

$\infty$
Freestream

Axial

Absolute, refers to the absolute pressure measurement from the mercury well manometer on the wall of the wind tunnel lab

Fan, refers to properties at, or by, the fan

Corrected

Center of gravity

At aftbody suction exit

Static

Total

Uncorrected

Wall, refers to the absolute pressure measurement from the mercury well manometer on the wall of the wind tunnel lab

Freestream 


\section{INTRODUCTION}

This document describes the wind tunnel tests of a bluff body with an integrated propulsion system that has come to be known as a Goldschmied propulsor. The concept, originally made famous by Fabio Goldschmied, is a fuselage with an embedded propulsion unit in the aft section of the body. The notable feature of the concept is the complementary combination of Boundary Layer Suction (BLS) and Boundary Layer Ingestion (BLI). BLS is a type of Boundary Layer Control (BLC) that removes the boundary layer through either distributed or concentrated suction to reduce flow separation and increase pressure recovery on the aft sections of relatively bluff bodies. BLI is when the relatively slow moving air of the boundary layer is ingested into the inlet of the propulsion unit (propulsor) in order to increase its propulsive efficiency. Each concept alone has shown to offer at least small benefits under the correct conditions. Goldschmied is credited with realizing the complementary nature of the concentrated BLS and BLI concepts and combining them together into a single configuration.

\subsection{PURPOSE}

The Goldschmied propulsor concept is one that many people do not believe delivers the benefits that were claimed. It is this author's hypothesis that it does provide a measureable benefit. This benefit is defined as a reduction in the energy required to attain a given net axial force on the model. It is the objective of the current effort to experimentally assess and thoroughly document the performance of a Goldschmied propulsor. This effort will be deemed successful if a measureable benefit can be demonstrated true or if this perceived benefit could be demonstrated false. 


\section{BACKGROUND}

\subsection{DRAG, THRUST, AND NET AXIAL FORCE}

The net axial force of the model, or just axial force, can be either positive, negative, or zero. In this paper, the positive direction will mean the direction of travel when the body is moving forward. In all of the published reports concerning Goldschmied, the term "Drag" was used when actually referring to the net axial force on the model. However, the real source of confusion from many authors on this subject is when the term "Drag Reduction" is mentioned. Drag is classically the force on a body that is only dependant on the fluid density, speed of the body moving through the fluid, and the shape of the body. The body shape dictates both the drag coefficient and the reference area. The reference area is dictated by the body scale. To alter the drag of the body, one of these items must be changed. Of these items, changes in the speed, fluid density, or scale of the body are not classified as drag reductions or increases. Drag reduction is therefore the result of a change made to the shape of a body, and it results in a lower drag coefficient, as determined in a wind tunnel or equivalent. Classically, propulsion is the process where energy is consumed and a force is created in the positive direction, effectively canceling the drag force and making the net force more positive (or less negative depending on how one chooses to look at it). The central question is if energy had to be expended to cause this drag reduction, as in the case of the Goldschmied propulsor, then is this really drag reduction, or is it a form of propulsion since energy was consumed and it has the same end result? In the classical sense, this is not a drag reduction because there was energy used to create the effect and the shape of the body remains unchanged. Also in the classical sense, this is not propulsion because no force was created in the positive direction to counter the drag force in the negative direction. The energy was simply used to alter the drag force and make it less negative, and not counter it. There is no engineering terminology for this type of a scenario. Therefore, to try to keep the confusion to a minimum, the terms drag and thrust will only refer to direction of the axial force.

In an effort to bring some clarity to the situation, Drela published a paper on the subject. ${ }^{1}$ In it, he proposes a method of accounting that avoids this undefined situation and only deals with the relevant energy and power terms for a given situation. It was the intention to use this approach in this thesis's analysis. The method itself is presented to be a universal approach and leaves the details of its implementation to the reader. However, after getting familiar with the approach, the depth and complexity of the method became apparent. These complexities lead this author to derive a much simpler force and energy accounting. Besides this complexity, Drela's paper claims that the drag 
force on a simple unpowered body is a function of the velocity cubed and not squared. This claim is a result of the method proposed, and since the claim goes against fundamental aerodynamic concepts that are the subject of countless volumes of reviewed and published literature, it was deemed safer not to follow Drela's proposed accounting method.

\subsection{REYNOLDS NUMBER}

The Reynolds number, as shown in Eq. 2.1, is the ratio of the inertial forces to the viscous forces on a body and in a particular flow field. It is a function of the fluid viscosity, the flow speed, and a reference dimension for the situation. This reference dimension usually is a significant dimension of the flow field. The most common reference dimension is the length of the body under test. The reference dimension for most of the previous Goldschmied work was the $1 / 3$ power of the body volume. ${ }^{2}$ This was done to keep with standard practice for airship design. Since this no longer pertains to airship design only, a more traditional reference dimension is used for this report. The reference dimension is the body length. The reference area will be the frontal area.

$$
R e=\frac{\rho V_{\infty} L}{\mu}
$$

\subsection{OVERVIEW OF ORIGINAL GOLDSCHMIED CONCEPT}

There have been three previous wind tunnel tests of a Goldschmied propulsor. These three tests are the only public verification of the concept. Goldschmied began this concept when he published a currently unavailable paper on the concept in 1955 with some preliminary analytical performance estimates. This led to a wind tunnel test to attempt to validate the theory in 1956, the results of which were published in two papers in 1957.,4 The two following tests were in 1969 and 1987..$^{5}$ Although, all tests used the same basic model, the later tests made small but important changes to it, as will be outlined in the following sections.

These tests were performed many years ago. The basic model was designed and built over 50 years ago. Many of the reports from these tests are either unavailable or missing critical information required to judge their credibility and duplicate their results. This has led some to discount the theory and test data as just creative accounting of the true behavior. In addition, the available test reports document a few problems encountered during the tests that invoke further speculation. 
In these previous tests, the BLS side of the concept has been investigated while the BLI side has been mostly ignored. This author believes this was due to a few reasons. First, the benefits from BLS were expected to be large in comparison to those from BLI and therefore the BLI benefits were completely ignored. Second, the propulsor, and integration thereof, were far less than ideal or did not represent a real-world practical system. Therefore, to analyze the propulsive efficiency of a non-ideal propulsor was probably deemed not worth the effort. Lastly, as most wind tunnel test programs do, these tests face many difficulties while testing. These difficulties include funding shortages, schedule shortages, and test apparatus problems. It is possible that at least one of the completed tests meant to analyze the BLI benefits but simply ran out of time and/or money. This current effort will again focus on the BLS side of the concept, as these same issues were characteristic of the present project as well.

It is the goal of the following sections to familiarize the reader with the model, test outline, and objectives of the original tests. The details of these original tests are given to provide background and, most importantly, context to the current work.

\section{$2.4 \quad 1956$ TEST}

The wind tunnel test that took place in 1956 was originally planned to take 8 weeks and test multiple body shapes, slot configurations, fan configurations, and fan settings. The main goal was to put forward a compelling case to NACA of the need for further development of analytical and empirical tools for the development of BLS and BLI systems. Unfortunately, the budget and tunnel time restrictions allowed only a 10-day test of one body, one basic slot configuration, and one fan configuration. The origin of this experiment was based on some analytical performance estimates that had been made by Goldschmied and others. ${ }^{6}$ The result of the experiment was to give only a binary 'yes or no' answer to the question of effectiveness of the concept, to which the answer was a conclusive yes. The model for the test was a scale model of a conceptual $340 \mathrm{ft}$ long airship. The test took place at the slotted wall wind tunnel at the David W. Taylor Model Basin (DWTMB). The test is described by Cerreta and the Goodyear Aircraft Corporation. ${ }^{3,4}$ It is from these two resources that all of the following test details were found.

\subsubsection{MODEL DESCRIPTION}

The shape of the wind tunnel model that was built for this test was one that had shown the most promise in the early potential flow based empirical estimates. The model surface shape originated as a Lighthill-designed airfoil. This airfoil had originally been developed for 2-D BLS and natural laminar flow research. This type of airfoil 
was chosen because it already had a single suction slot on its surface and a large region with a favorable pressure gradient upstream of the slot. It was designed using Lighthill's inverse design procedure for a 2-D high Reynolds number case within an angle of attack range of $\pm 7^{\circ}$. Even though the Goldschmied propulsor did not use laminar flow for its drag reduction, this large region with a favorable pressure gradient was thought to be important because it would prevent the turbulent boundary from growing too large before it could be removed adequately by the BLS system. The specific airfoil selected was the $34 \%$ thick Lighthill airfoil. From this airfoil, the profile was revolved around a centerline to produce the basic axisymmetric shape. This shape however was not the final shape to be tested.

The model designers placed the 2 dimensional profile of the shape into what they called an electrostatic tank. This was done to predict the 2-D potential flow field surrounding the body. It used a static electric field produced by an AC voltage between the model profile and an electrolyte solution surrounding the model profile. This electrolyte solution contained ferrous material that aligned with the local magnetic field. From this method, the inviscid flow field and pressure distribution was approximated. In the electrostatic tank, the flow was assumed attached because of the inviscid and incompressible potential flow assumptions, and not because of the sink effect of the concentrated BLS. It is worth noting that this potential flow field suffers from D' Alembert's paradox of zero drag. This was evident in the calibration of the electrostatic tank, which entailed a cylinder with an almost perfect potential flow field around it. Modifications to the profile were made in order to eliminate excessive velocity gradients and create a smooth pressure distribution. One of these modifications can be seen in Fig. 2-2. Once the modifications were made in 2-D, a "three dimensional" velocity distribution was found by tilting the tank $10^{\circ}$ and further modifications to the profile were made. The model shape that resulted from the two electrostatic tank tests, was the final model shape used in the wind tunnel tests. The general configuration and dimensions for this model can be seen in Fig. 2-1. ${ }^{2}$ 


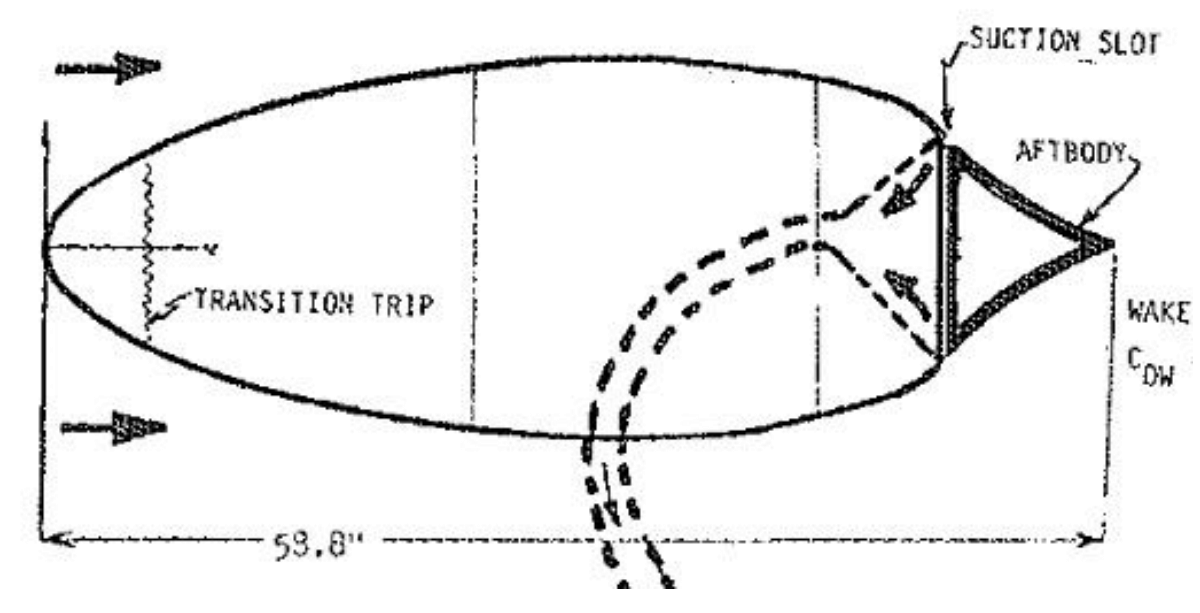

Figure 2-1. General configuration of the model used in the 1956 test of a Goldschmied propulsor. ${ }^{4}$

Although the details of the electrostatic tank method are lacking, the intent of it was to present a body for wind tunnel testing that was good enough for a proof-of-concept. The shape was never "optimized" for any conditions because the potential flow analog of the tank does not suffer from any viscous effects. The body was meant to be redesigned in future efforts when sufficient methods, time, and means were available. This point is important to keep in the reader's minds when looking at the previous and current data. All of this data is from a body that was designed more than 50 years ago with techniques that are no longer used and are marginal, at best, in their results due to the relevance of their assumptions. These techniques did not take into account any viscous effects or even the fact that the body is axisymmetric. It is a testament to the credibility and promise of the Goldschmied propulsor concept that it can be applied to a body through such crude techniques, by today's standards, and it still obtained such promising results. The following is stated about proper context for this test.

"The effects of such a minimum schedule were sorely felt. Considerable time and effort was consumed during the test in attempting to 'quick fix' a basically inadequate program. Nevertheless, the results, when viewed in the proper perspective, were encouraging and indicated acceptable agreement with the available theories ( ). Furthermore, these results should greatly influence the direction of future work."

The wind tunnel model did not contain the fan that supplied the suction flow. The model routed the suction flow through the model strut, through a calibrated venturi meter, then through the fan, and exhausted it out into the tunnel room. The aftbody came to a cusp at the trailing edge. The experiment included tests of two different slot geometries, and another external annular airfoil geometry that was similar to a duct or shroud around the aftbody. 
The two different slot geometries performed identically and showed a measureable drag reduction. The duct geometry showed a severe drag penalty. These three geometries can be seen in Fig. 2-3.

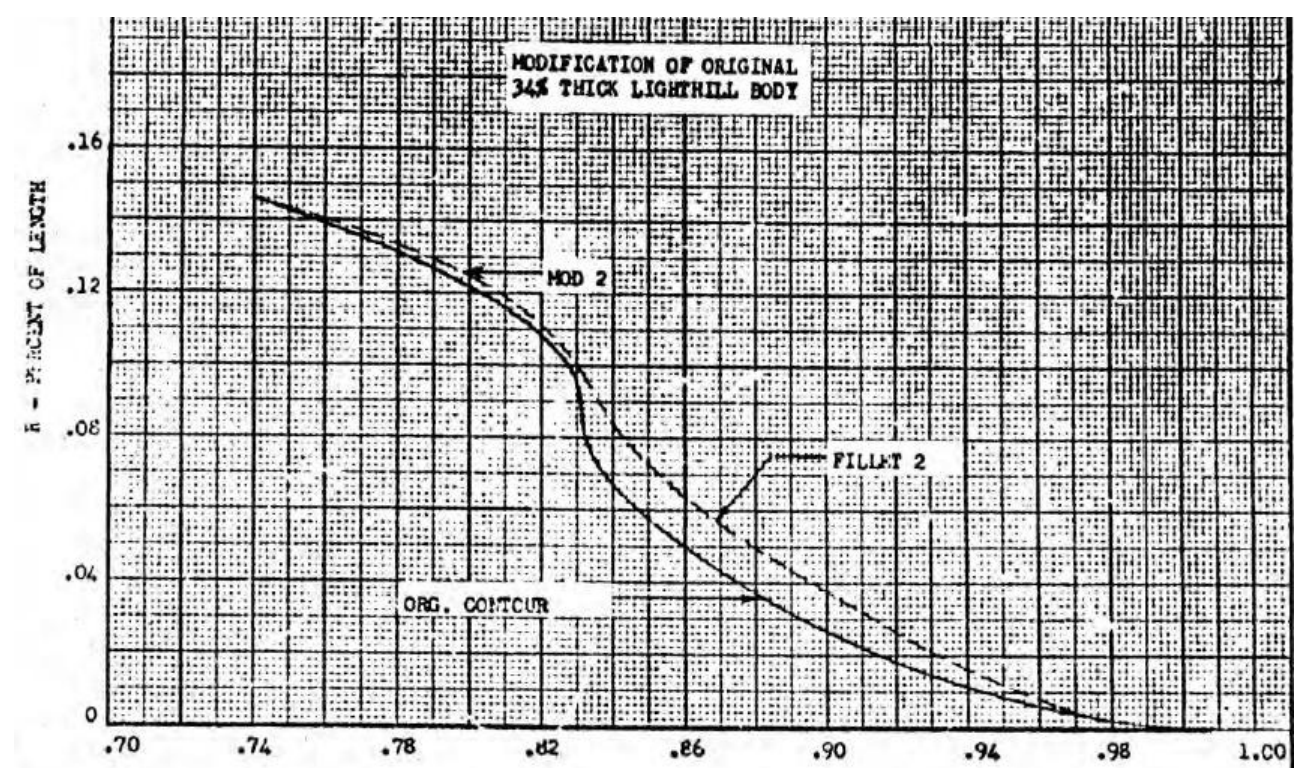

Figure 2-2. One of the aftbody modifications to the Lighthill $34 \%$ thick airfoil which resulted from the use of the electrostatic tank. ${ }^{4}$

The model nose and tail were manufactured by "spinning" aluminum. This is an amazing process not too different from that done by a pottery sculptor with clay. It allows a flat sheet of aluminum to be contoured through brute force into an axisymmetric shape while it is spun on a lathe. The rest of the model was made of fiberglass with aluminum bracing. The aft body was attached to the forbody through three rods and a mechanical drive mechanism. This mechanism allowed the aftbody to move relative to the forbody. This relative movement is the means by which the slot width was adjusted. 


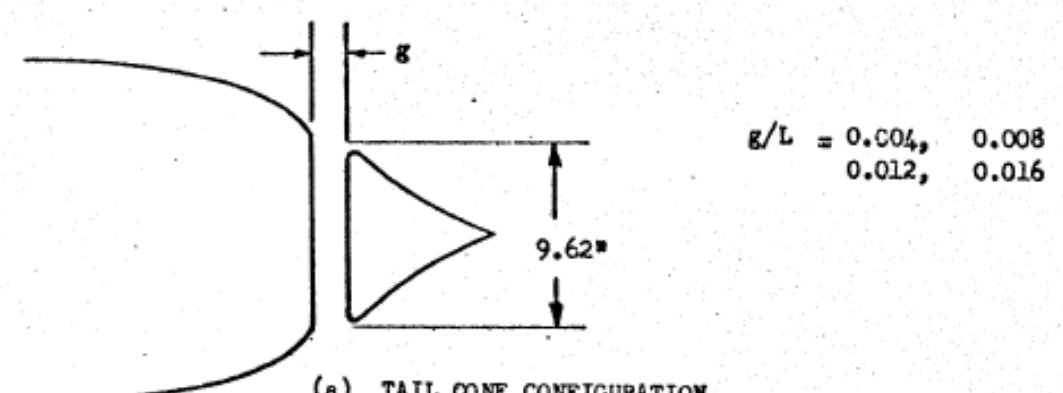

(a) TAIL DONE CONFIGURATION

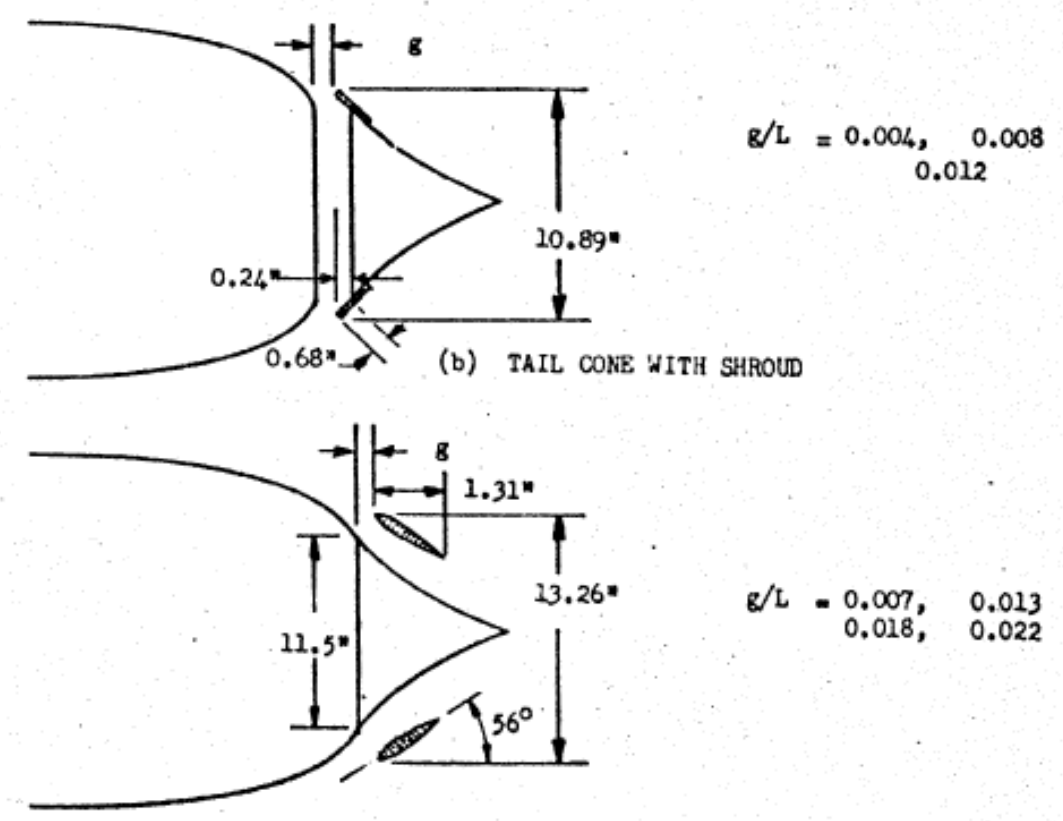

(c) ANNUiAR AIRFOIL CONFIGURATION

Figure 2-3. A schematic of the three aft body configurations tested during the 1956 test of a Goldschmied propulsor. ${ }^{4}$

The drag for each test point was measured with a large wake rake in the shape of a "+" sign. The wake rake contained up to 167 total pressure probes and 7 static pressure probes. This allowed the ability to capture the entire wake profile on the manometer rack with adequate resolution as well as account for any non-symmetry in the wake. The wake rake was adjusted for each run to be in the center of the wake both horizontally and vertically. The measured wake profile was then integrated to determine the total drag at that particular test point.

Surface pressure was measured at 43 locations on the body. The ports were on the horizontal plane of the model and ran down both opposing sides with 21 ports on each side. The axial spacing of the ports differed from side to side to create one continuous profile based on data from two sides of the model. Two boundary layer rakes 
were used to measure the velocity profiles upstream and downstream of the suction slot. These were used to calculate boundary layer parameters for later analysis.

\subsubsection{DEFINITION OF SUCTION DRAG}

The data reduction methods used in this test were very scarcely explained or justified. One important parameter is what was called "suction drag" which is described by Cerreta in the following way.

"the suction pump drag coefficient, $\mathrm{C}_{\mathrm{D}, \mathrm{s}}$, was determined from energy balance considerations in which the pump work required to restore that portion of the boundary layer that was withdrawn by the suction system to freestream total head is converted to an equivalent drag.",

In this method, the measured velocity profile just upstream of the suction slot was used to calculate the height off the surface of the body that contained the same mass flow as was measured by the flow meter for the suction flow. The power was then calculated that would isentropically restore the total pressure (referred to in the quote as total head) deficit below this height to freestream total pressure. This calculated power required to restore the suction flow back to freestream total pressure was divided by the freestream velocity and non-dimensionalized by the test dynamic pressure and the $2 / 3$ power of the model volume to calculate $C_{\mathrm{D}, s}$, as can be seen in Eq. 2.2.

$$
C_{D, s}=\frac{W_{s}}{U_{\infty} q_{\infty}\left(V_{B}\right)^{2 / 3}}
$$

The concept of suction drag is fundamental in understanding the approach used, and maybe some of the reasons for the extraordinary claims made as a result. The basic concept of suction drag can be understood through the relationship between power and force. If the power consumed by the suction system was used instead to create thrust through a traditional propulsor, suction drag is the amount of drag that this thrust would counteract. It fundamentally converts the power used for suction to an equivalent drag that if present, would have resulted in the same amount of total power consumed.

Another way to think about it is in terms of the power required to maintain steady level flight. Any given aircraft requires a certain amount of power to fly at a constant speed under given freestream conditions. This power is simply the product of the aircraft speed and drag, assuming that the thrust equals the drag. Assume now that the drag decreased due to a configuration change on the aircraft, like raising the landing gear. The aircraft now requires less power to fly the same speed because the drag, and thrust, has decreased. The change in power is just the product 
of the freestream speed and the difference between the amount of drag before and after the change. However, say for instance, that the pilot only knew what the change in power was due to a gauge for the engine, such as a fuel flow meter. The pilot could calculate the amount of drag difference from raising the landing gear. This is in essence what was done in this 1956 test, the change in power was calculated, and the change in drag was determined from this power difference and the freestream speed.

There is one important difference between the previous analysis in the landing gear example and that made by Goldschmied. In the above landing gear example, the pilot might need to know some of the details of the intermediate parts of the propulsion system to estimate the power used. Goldschmied did not account for any intermediate component efficiencies in his conversion between the suction power and how much drag that power could counteract. Doing this is admittedly difficult because it is completely dependent on the details of the system, which are not known, or even desired, for this level of research. In reality, this method of not accounting for the system dependent efficiencies sets an upper bound on the performance of the system and represents the 'best case' scenario.

One other shortcoming of the 1956 test is in the calculation of the power required. To be able to expel only enough power to restore the suction flow to freestream total pressure, effectively filling in the velocity profile and increasing static pressure to that of freestream, would be a very difficult task, and to do it isentropically would be impossible. It is true that by filling in the lost momentum in the model wake, the minimum power is used to achieve a given net axial force, but this is not practical. No device exists that could take a non-uniform velocity profile and make it uniform while increasing the static pressure to be uniform as well. The reported pressure drag, mass flow and other measured parameters were a result of probably much greater power consumption than is represented by this calculation.

\section{$2.5 \quad 1969$ TEST}

This wind tunnel test changed the design vehicle from an airship to an underwater vehicle using the Goldschmied propulsor concept. It used the same model as the 1956 test with the addition of many different suction slot designs. It was also tested at angles of attack up to $6^{\circ}$. From the very little information that is published in other reports, the specific goal was to determine the geometric parameters of the suction slot that had the greatest effect on the model performance. It is noted that the funding for the test was cut before the propulsion unit could be installed 
in the model. It is assumed that this means the plan for the model was to incorporate a fan/propulsor unit into it. The model configuration, test outline, and data are largely unavailable except for the few data points in the plot in Fig. 2-4. As can be seen, equivalent drag levels between the 1969 and 1956 tests were seen at much lower suction flow coefficients. These two data points are the only available from this test. Also shown in the diagram is the comparison of the data to a theoretical minimum power required to achieve full attachment. Also included for comparison are some relevant airship drag coefficients from the era and some data points from the 1956 test. One interesting thing to note from this figure is the magnitude of the wake drag, which were roughly 50 drag counts.

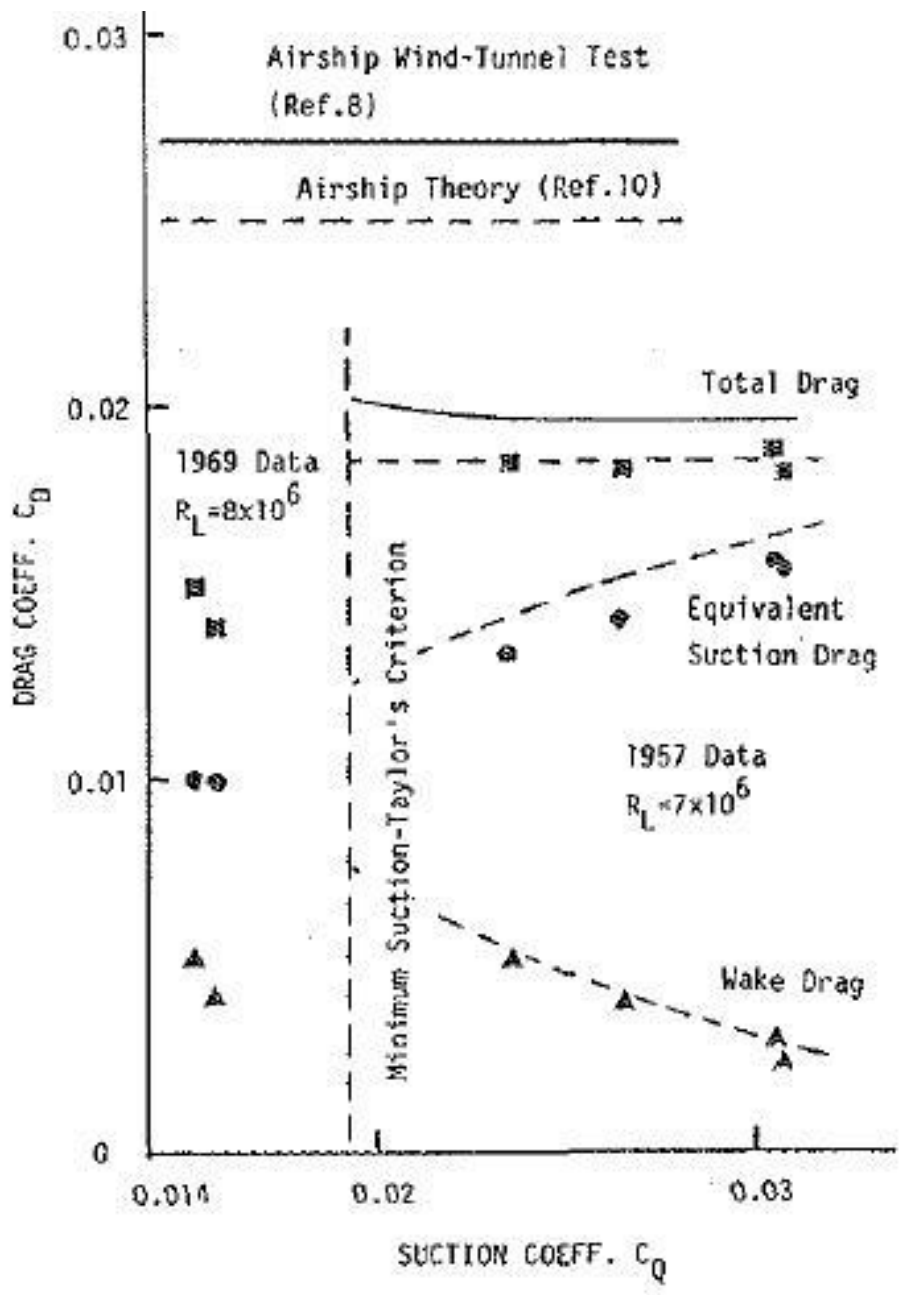

Figure 2-4. The only available two data points from the 1969 test of the Goldschmied propulsor compared against data from the 1956 test. $^{8}$

One last thing that is known about this test is that the best performing cusp was called the "Ringloeb" cusp. This cusp was assumedly named after Frederick Ringleb because of a citation to a Ringleb paper ${ }^{9}$ in a Goldschmied 
paper ${ }^{10}$ discussing the cusp's performance benefits. Frederick Ringleb's paper discusses trapped vortices and their ability to help a flow turn a sharper corner than if the corner was a rigid surface. The paper includes flow calculations and justifications based on potential flow theory, and it does not mention any viscous effects. Therefore, the leap between the Frederick Ringleb paper and Goldschmied's misspelled Ringloeb cusp is unclear.

The Ringleb paper does include a short discussion of the combined effects of suction with a trapped vortex, but the paper lacks a lot of detail in this discussion. The one airfoil with a trapped vortex and trailing edge suction that is discussed briefly in the paper is shown in Fig. 2-5. This airfoil uses suction to create lift. At a zero degree angle of attack and a given flow coefficient, the airfoil has a lift coefficient of 0.096. Then when the suction flow coefficient is increased slightly, the lift coefficient increases to1.275. It is cited that this is from data of a wind tunnel test done at Princeton and presented publicly in 1953.

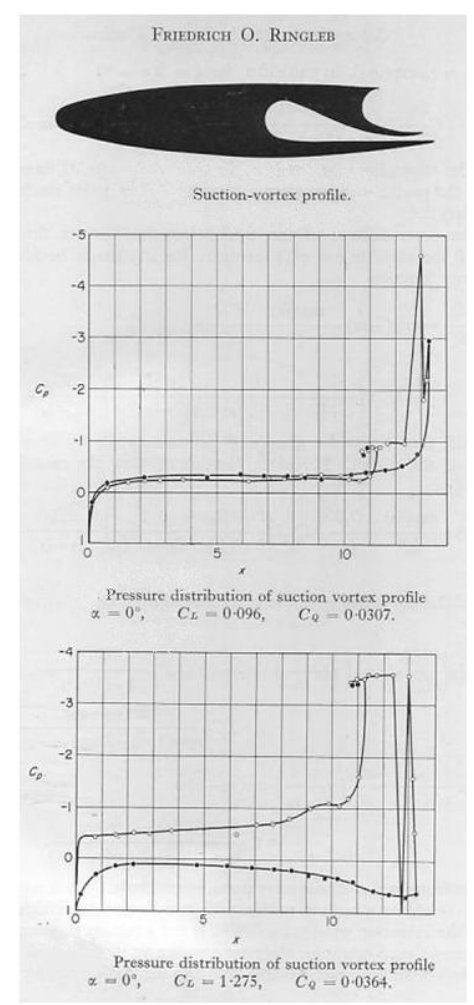

Figure 2-5. Depiction of a suction slot airfoil that uses a trapped vortex to help the suction flow to smoothly enter the suction slot. ${ }^{9}$ 


\subsection{TEST}

This test was by far the most extensive of the three tests. It is the most cited test in the published papers on the subject. One source ${ }^{5}$ calls the Goldschmied propulsor concept the SAP, or Suction Aftbody Propulsor concept, and another source ${ }^{11}$ simply call it the Goldschmied model. These are the two main test reports where all of the following details were gathered. The main goals of this test were to incorporate the fan/propulsor inside the model and test the performance and static stability with a simple cruciform tail. The maximum test Reynolds number achieved with full attachment on the aftbody of the length was 3.2 million with transition tripped at $10 \%$, and it was 4.1 million with transition not tripped. The summary of the test given here will focus on the differences between the 1982 test and the previous tests.

It used the same basic model as in the previous tests, except it completely redesigned the aftbody to incorporate a dual stage counter-rotating fan. The $27 \mathrm{Vdc}$ fan was rated at $600 \mathrm{CFM}$ (cubic feet per minute) at a pressure rise of $0.396 \mathrm{psi}$, and $700 \mathrm{CFM}$ at $0.343 \mathrm{psi}$. It used the best performing suction slot from the previous test, the Ringleb cusp, for all test points. The aftbody sections were machined from billet aluminum. There were two aftbody configurations tested. The shorter section was used alone, while a longer aftbody was used with the cruciform tail in the stability testing. These aftbodies can be seen in Fig. 2-6 and Fig. 2-7, followed by a detailed schematic of the model, its instrumentation, and dimensions in Fig. 2-8 and Fig. 2-9.

The test matrix contained 5 variables and over 800 data points. These variables were suction fan speed, tunnel airspeed, aftbody configuration, yaw angle, and trip strip location. It included points with transition tripped at 10\% and $59 \%$ of the body length, as well as free transition cases. The fan speed was varied from $0-100 \%$. The yaw angles tested were $0^{\circ}, 3^{\circ}, 6^{\circ}$, and $8^{\circ}$. The dynamic pressures tested were $15 \mathrm{psf}, 20 \mathrm{psf}, 25 \mathrm{psf}$, and $35 \mathrm{psf}$. It was noted that the maximum value of the dynamic pressure correction due to blockage, buoyancy, and compressibility was a total value of $4 \%$. 


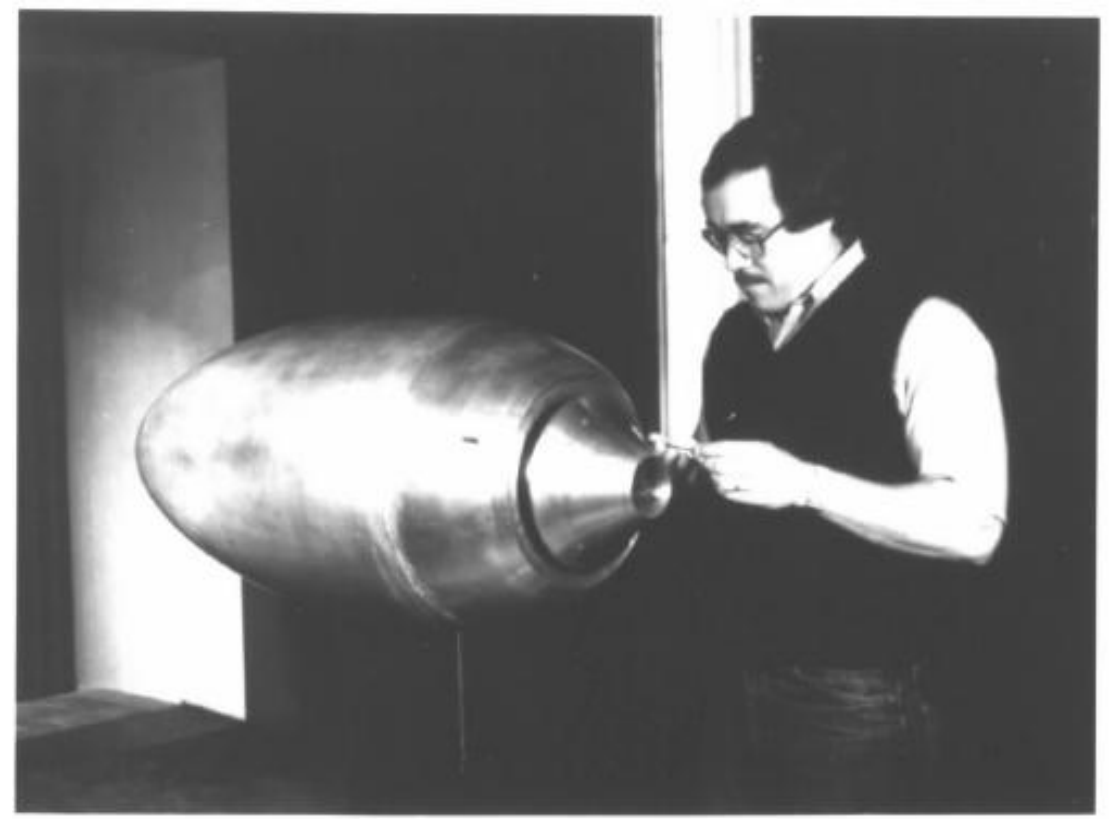

Figure 2-6. Fabio R. Goldschmied and the SAP model with the short aftbody as used in the 1982 wind tunnel test. $^{5}$

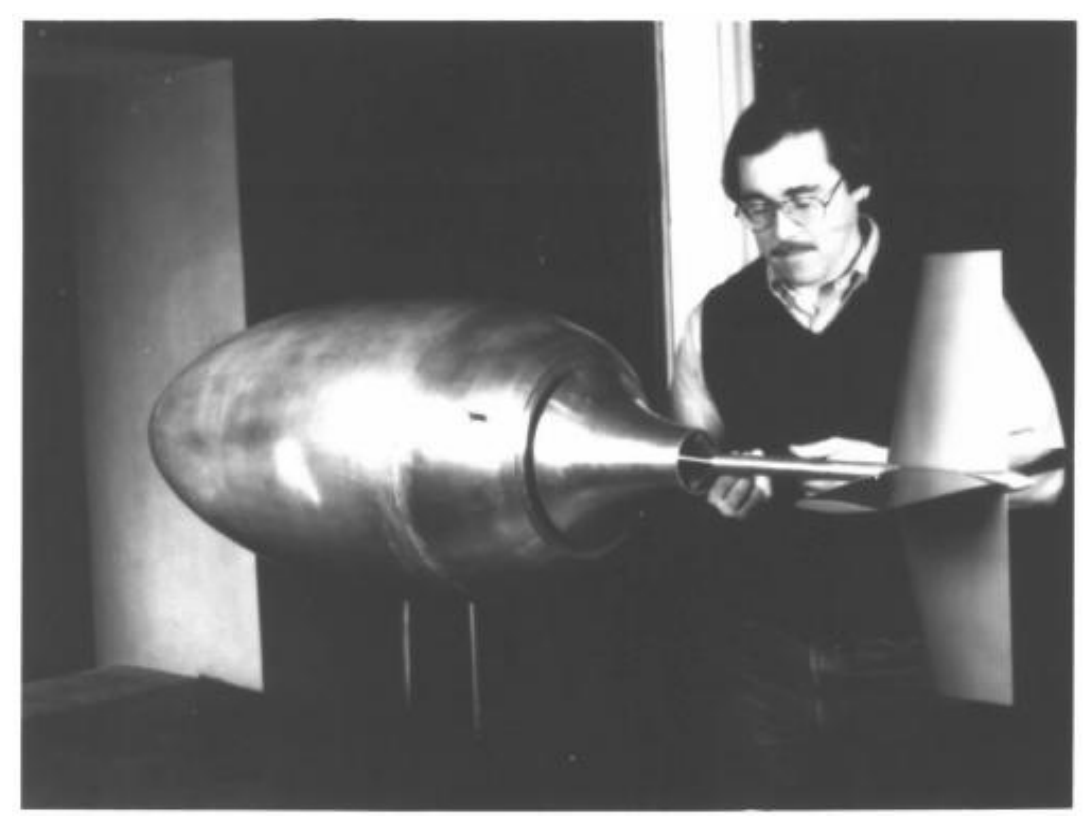

Figure 2-7. Fabio R. Goldschmied and the SAP model with the long aftbody and cruciform tail as used in the 1982 wind tunnel test. ${ }^{5}$ 


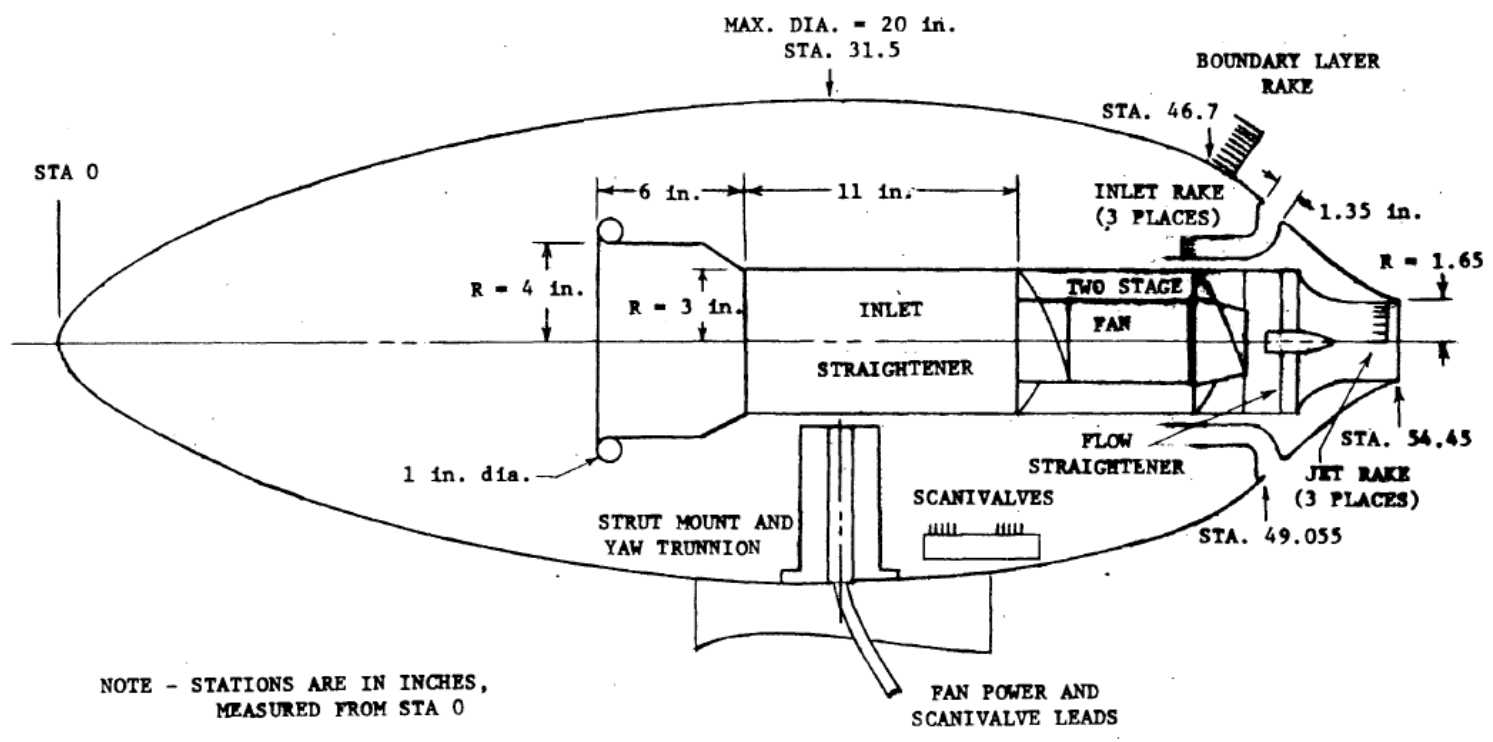

Figure 2-8. Schematic of the SAP concept that shows the locations of the boundary layer rake, the fan $\&$ flow straightener devices, as well as the general dimensions of the body. ${ }^{5}$

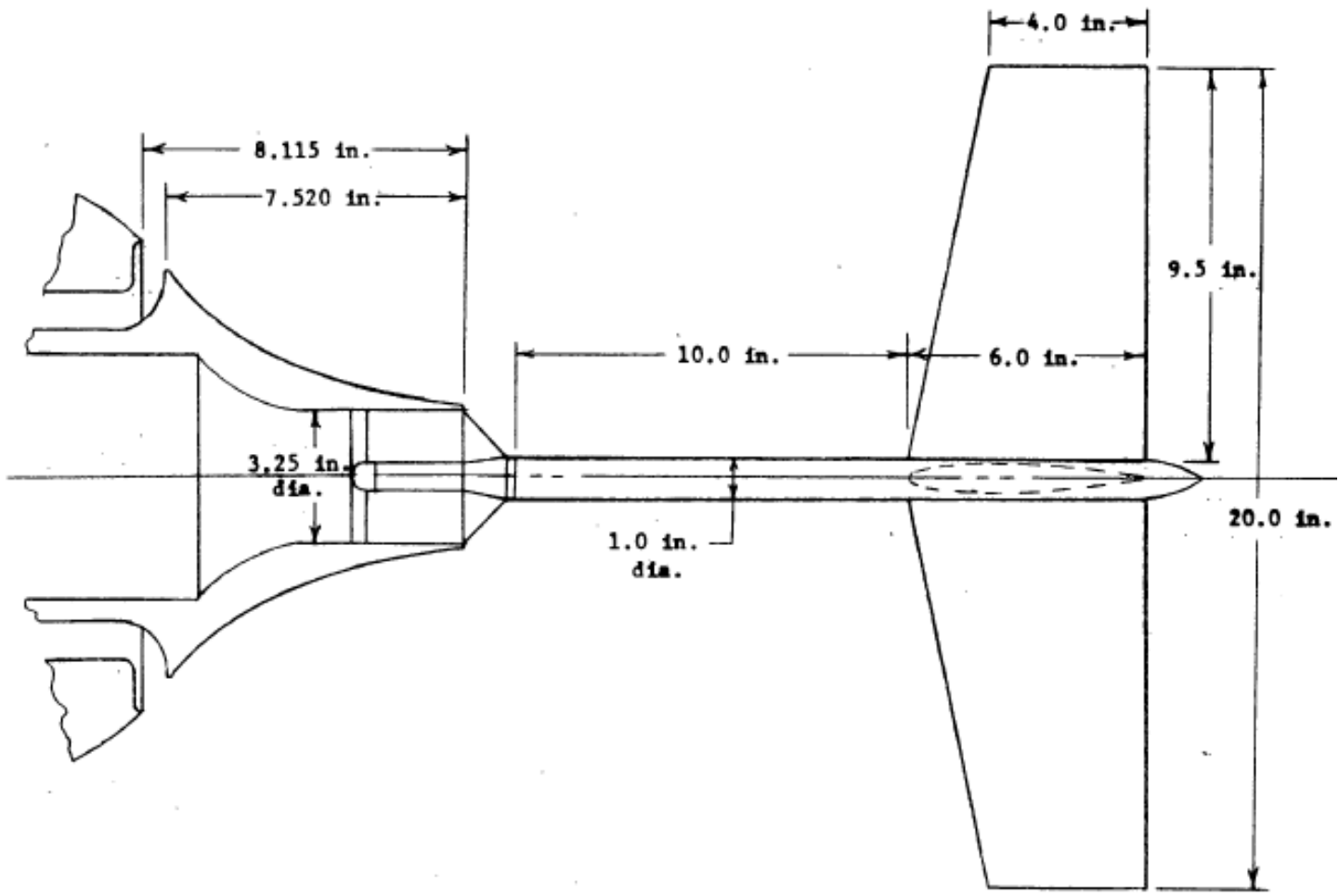

Figure 2-9. Schematic of the long aftbody with the tail and associated dimensions. ${ }^{5}$ 


\subsubsection{TeSt Challenges}

The testing did not go as smoothly as hoped for this important test. The main issues seen by the operators were high inlet losses at the entrance to the slot and fan, model wake entrainment due to the wake of the strut, and the strut wake interfering with the model's ability to establish flow attachment on the lower aftbody.

The inlet losses at the entrance to the fan were significantly higher than expected by the test designers. Consequently, the inlet losses caused the fan power to be higher than it otherwise would have been. This resulted in the unfortunate circumstance that the fan could not provide a high enough suction flow rate to achieve attached flow at any higher tunnel dynamic pressure than 15 psf when the boundary layer was tripped at $10 \%$ of the length. As an attempt to fix this problem, the normal cusp was removed from the slot and a smaller hand-formed clay cusp was fitted. This clay cusp provided a smoother flow path into the slot and it performed well, but not as well as the Ringleb cusp.

In addition, it was observed during flow visualizations that interference from the strut/fairing resulted in the flow on the bottom side of the model achieving flow attachment at a higher suction flow rate and fan power than the upper surface. This resulted in higher values of fan power and suction flow rate to achieve fully attached flow. The wake of the strut also caused a second problem. It is the view of the test operators that the wake of the model was entrained and pulled downward by the wake of the strut. This caused a misalignment between the wake and the wake rake that could not be remedied. This explains why the force measurement from the wake rake disagreed with the balance measurements until a high level of suction flow. This strong influence of the wake from the strut was seen in tuft flow visualizations.

Also of interest is the notation that the short aftbody was harder to maintain flow attachment than the longer aftbody, but the shorter aftbody required less power to establish this attachment.

\subsubsection{TEST DATA REDUCTION}

The following is the general method used to reduce the data in this test. The model was well instrumented. It heavily relied on pressure measurements for many parameters. It used three rakes in the suction path to measure mass flow rate. This mass flow calculation even went so far as to linearly interpolate the local static pressure between the inside of the duct's surface pressure port and static probes in the rakes. There was also an 18-probe boundary layer rake just upstream of the suction slot on the top of the model. Once the mass flow was measured, the 
boundary layer rake data was used to fit a curve to the velocity profile and then the height off the surface that encompassed the ingested suction flow was determined. Using the approximate velocity profile, a head loss coefficient was determined between freestream and the air in the boundary layer at the location of the boundary layer rake.

At the exit to the suction flow path, the exit velocity was determined from the average total pressure at the exit and the freestream static pressure measured upstream. It describes this as the "hypothetical far-downstream expanded velocity." In the wake of the model, a numerically integrated axisymmetric Jones equation was used to calculate the total axial force on the model.

The balance measurements were considered more accurate due to the issues with the alignment of the wake rake. The balance measurements were corrected according to Eq. 2.3. The constants A and B were different for either "laminar or turbulent data runs." ${ }^{2}$ The $\mathrm{D}_{\mathrm{u}}$ term is the uncorrected drag value. It is not known what is meant by laminar or turbulent data runs. It is assumed that this is referring to runs where the boundary layer was tripped at different locations on the model, i.e. $10 \%$ would be a turbulent run, and free transition would be a laminar run. It is unknown if these had to do with the flow over the strut and fairing, or flow over the model.

$$
D=D_{u}-\left(A q_{\infty}+B\right)
$$

The power calculations are probably the most interesting. The power required to power the fan was not measured or used at all in any of the calculations. The power required, $\mathrm{C}_{\mathrm{p}, \mathrm{t}}$, has two terms. The first term, $\mathrm{C}_{\mathrm{p}, \mathrm{s}}$, is derived and calculated in a very similar manner as the 1956 test. It is conceptually the power required to increase the total pressure in the suction system back up to freestream total pressure, and is based on the pressure loss just upstream of the fan and the suction flow's volume flow rate. In coefficient form, it is shown in Eq. 2.5. The second term, $\mathrm{C}_{\mathrm{p}, \mathrm{p}}$, is the "remaining added fluid power for self-propulsion." ${ }^{2}$ This is meant to be the power required to increase the exit velocity enough to reach zero net axial force.

$$
\begin{aligned}
& C_{p, t}=C_{p, s}+C_{p, p} \\
& C_{p, s}=C H_{02} * C_{Q}
\end{aligned}
$$




$$
\begin{gathered}
C H_{02}=\frac{\left(P_{t, \infty}-P_{t, 2}\right)}{q_{\infty}} \\
C_{Q}=\frac{Q}{V_{\infty} A_{\text {ref }}} \\
C_{p, p}=C_{D e x}\left(1+\frac{C_{D e x}}{4 C_{Q}}\right) \\
C_{D_{e x}}=2 C_{Q} \frac{u_{e}}{U_{\infty}} \\
u_{e}=\frac{D_{e x}}{\dot{m}} \\
D_{e x}=D-\dot{m}\left[U_{\infty}-u_{j}\right] \\
u_{j}=\sqrt{\frac{2\left(P_{t, e}-P_{\infty}\right)}{\rho}}
\end{gathered}
$$

Following the data reduction description is the following statement regarding the data reduction process. It is interesting that in the context of a wind tunnel test meant to validate a concept that the author would make such a statement.

"The data reduction procedures outlined above and reflected in the results plotted in Appendix B [of the cited paper] involve a number of somewhat arbitrary choices and assumptions. The results thus constitute one set of estimates of the performance of a vehicle presented by the model, and one set of many different sets of estimates, which could be made. In general, conservative choices and assumptions were made which would tend to yield less favorable estimates of the vehicle performance than are probably justified."2

This statement really brings into question the reliability of the data reduction process, especially when it comes to one of the more important parameters, fan power.

\subsection{TRANSITION TRIP STRIPS}

There exist three types of steady flow over the surface of any shape when a fluid is flowing over it. These types are laminar flow, turbulent flow, and separated flow. There is also a region of transition between laminar and turbulent flows, but this is a mix of the two flows instead of a distinct type. Countless volumes have been written 
about the characteristics of each type of flow. The transition between laminar and turbulent flow, and how it pertains to wind tunnel testing, is the focus of this section.

The transition trip strip has two main variables when it is implemented on a wind tunnel model. The first is that it must be placed in the correct location on the model, and the second is that it must be of the correct shape and height to actually transition the flow to turbulent without affecting the overall drag force besides the forces caused by the turbulent flow. The transition trip strip is commonly placed to meet two requirements. The first is that the Reynolds number based on the freestream flow parameters and the axial distance to the trip strip is at least $100,000{ }^{12}$ In addition to this requirement, the trip strip is generally placed aft, or near the end of, any region with a favorable pressure gradient. This is because a strong favorable pressure gradient can cause the boundary layer to relaminarize. The trip strip must also be far enough forward to never be placed in an adverse pressure gradient.

The second requirement of the trip strip is the roughness Reynolds number based on local flow parameters just outside the boundary layer and the roughness height, shown in Eq. 2.13, are high enough that the flow will not revert to laminar flow, or relaminarize, yet small enough to not cause extra "grit drag". This minimum roughness Reynolds number is $600 .^{12}$ This dictates the height of the roughness. The shape of the roughness should be as "3D" as possible. Two dimensional trip strips, such as wires, are less effective at inducing turbulent flow.

$$
R e_{k}=\frac{u_{k} k}{v_{k}}
$$

For the current model, the transition location was held fixed at the same location for all test points. This was done for many reasons, the first of which is to replicate the test conditions of previous tests to allow a more direct comparison. All previous tests, except some test points in the 1982 test, transitioned the flow at $10 \%$ of the body length. The details of how this was done in the previous tests are not given in any of the test reports.

The second reason is to eliminate the relative ratio of laminar and turbulent flow as a variable in the total axial force measurement. The total force on a body can be separated into two sources. These are the skin friction force and the pressure force. The skin friction force is due to the air exerting a shear stress on the model surface as it passes over it. The pressure force is due to the change in velocity and pressure over the model surface. The skin friction force is largely dependent on the type of flow over the surface. For a flat plate with $100 \%$ laminar flow over it, the drag is a small fraction of the drag if it was $100 \%$ turbulent flow. Therefore, it can be reasoned that the drag 
over a streamlined object will be significantly different depending on the relative amounts of laminar or turbulent flow over the surface. As discussed previously, the transition location is dependent on many things, including the pressure gradient on the surface of the model. In the current test, one of the effects of the BLS system is the alteration of the pressure gradient on the model surface. Therefore, when the suction mass flow is changed, the transition location might move and the drag on the model could change. This change would not only include the effect of the BLS on the flow separation and pressure recovery near the suction slot and aft end of the model, but it would include a change due to the different proportion of laminar and turbulent flow. By fixing the transition location, this change in the model drag is effectively eliminated. 


\section{EXPERIMENTAL APPARATUS}

\subsection{CAL POLY WIND TUNNEL}

The wind tunnel in the Aerospace Engineering Department of Cal Poly, San Luis Obispo was used for this project. It has a rectangular test section that measures nominally 3' by 4' internally, or more precisely, 34 1/4” x 46 $3 / 8$ ". It is an in-draft through tunnel with a single 440 Volt 3 -phase 150 horsepower electric motor driving a fixedpitch 9-blade fan downstream of the test section. The electric motor's speed is controlled by a SquareD Altivar66 variable frequency drive (VFD).

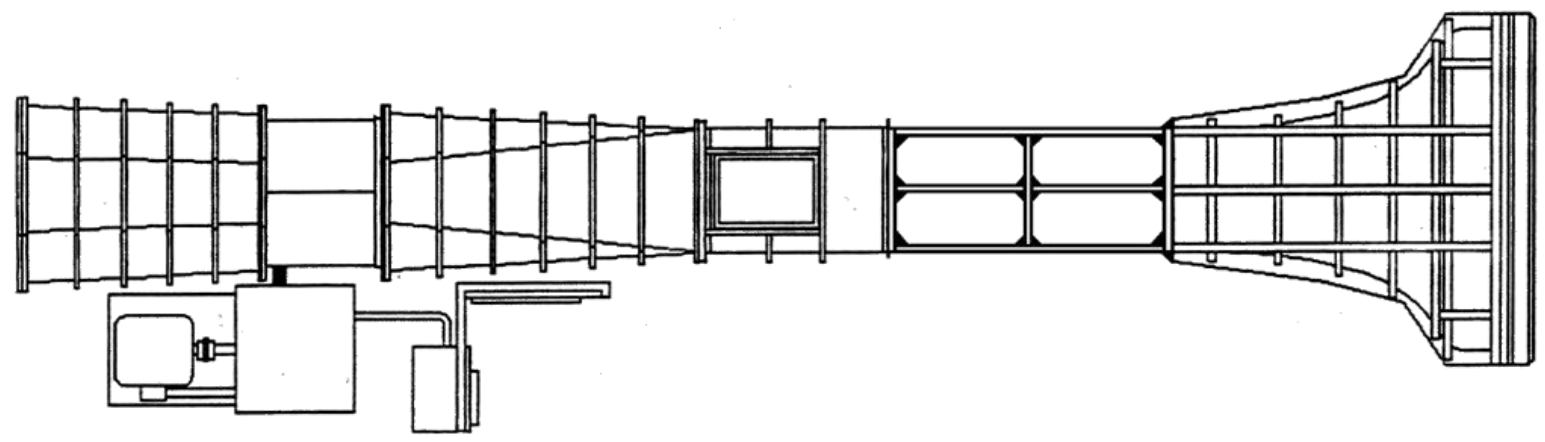

Figure 3-1. Layout of the Cal Poly Aerospace Engineering 3'x4' indraft wind tunnel.

\subsubsection{Cal Poly Wind Tunnel History}

This wind tunnel was built starting in 1974 by Professor Jon Hoffman, other faculty, and students. It is constructed almost entirely out of wood. The wind tunnel is meant to be reconfigurable with test sections that can be swapped and added to allow a wide range of experiments. It also has a permanent test section that houses a sting balance from Aerolab. The original swappable test sections are two 5' long test sections that could be used either together or independently. The wind tunnel was structurally and functionally designed to have a peak velocity of $100 \mathrm{mph}(44.7 \mathrm{~m} / \mathrm{s})$, but is currently only capable of achieving $78 \mathrm{mph}(34.9 \mathrm{~m} / \mathrm{s})$ with the current fan unit. The wind tunnel was housed inside the hangar, Building 4, until about 2005 when it was moved to its current location, Building 41.

One interesting design feature of the original wind tunnel design that did not make it into the actual wind tunnel was chamfers in the corners of the test sections that were to allow the test section area to be increasing along the test section. By omitting these chamfers, the boundary layer growth causes the freestream flow to converge and 
accelerate slightly through the test section. This acceleration of the flow causes the dynamic pressure to increase and the static pressure to decrease in the downstream direction. These effects must be corrected for and will be discussed further in Section 4.2.2.

A few years before the wind tunnel was moved out of the hanger, a new test section was added to the tunnel. This test section was originally built for, and used in, a NASA 3' by 4' wind tunnel. This test section consists of extruded aluminum T-slotted framing and Plexiglas. A very beneficial feature of this test section is the longitudinal slot that runs the length of this test section on the left side. The slot allows probes, instruments, and smoke-wands to be inserted through it into the test section at a wide range of longitudinal locations. On the outside of this slot is additional framing which hold a large traverse setup. The traverse setup consists of two Isel stepper motor traverses that are mounted to allow movement longitudinally and transversely with respect to the test section. An airfoil-shaped probe support is also part of this setup to hold the probes inside the tunnel as well as shield any wiring or tubing until it exits the tunnel. The slot through which the probe support enters the tunnel contains foam and weather-stripping. This weather-stripping in pushed aside by the probe support as it moves in the slot, but returns to its original shape to seal the slot when the probe support is no longer in that location. This test section can be seen in Fig. 3-2. 


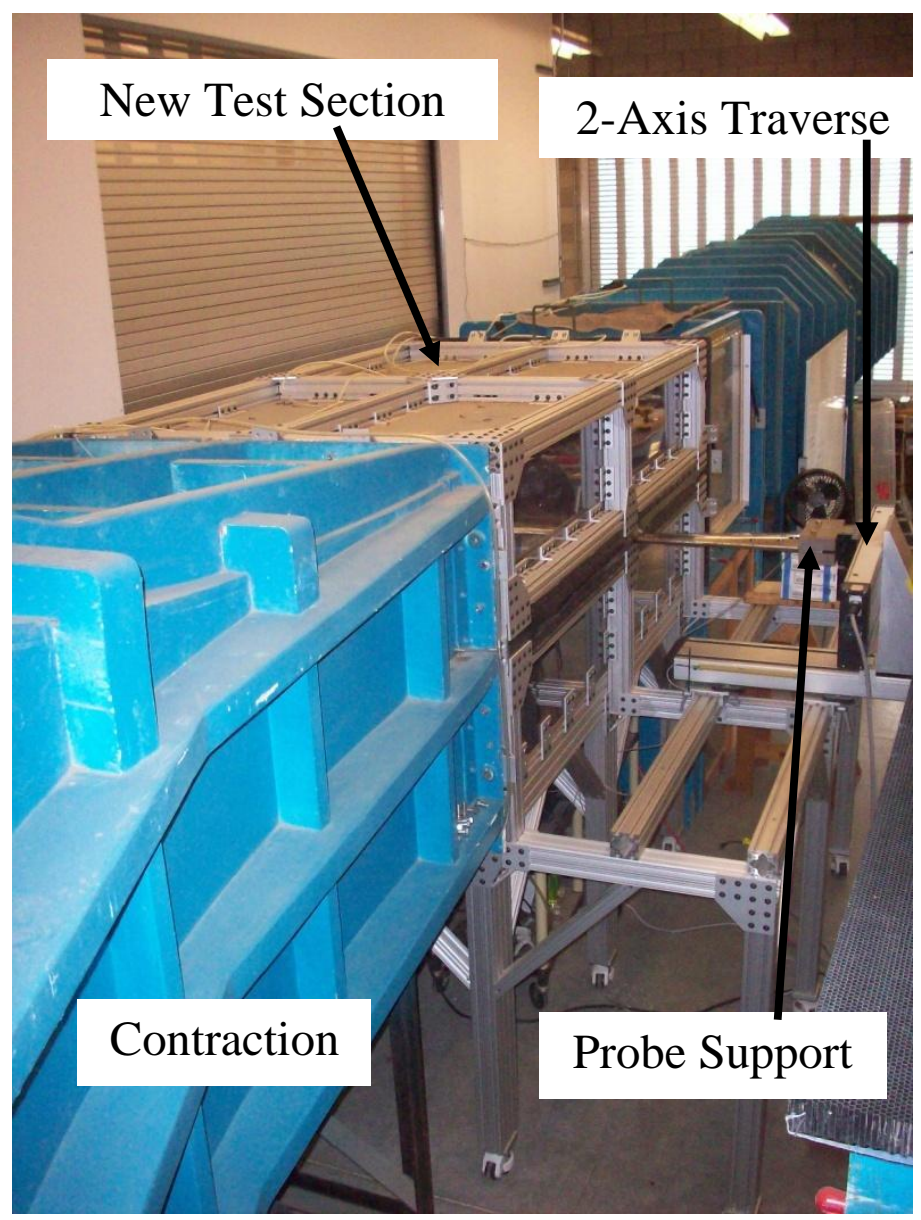

Figure 3-2. The aluminum and Plexiglas test section and accompanying traverse that was used in the current testing.

\subsubsection{Cal Poly Wind Tunnel Recent Modifications}

In its current location, the wind tunnel draws air in from vents in the roof of the building, and exhausts that air out a large rollup door in the far wall of the room. Through regressive testing in the wind tunnel during the past few years, the flow quality of the wind tunnel was discovered to be degrading. An exhaustive diagnosis process was undertaken by the author and some fellow graduate students and the flow quality was determined to be suffering from significant blockage in the flow straightener. As a result, the inlet to the wind tunnel was completely redesigned and rebuilt with new screens and a honeycomb flow straightener. The flow straightener originally consisted of four 18-mesh screens (18 openings per inch). Between the first and second screens were ordinary drinking straws used as a flow straightener. The straws were about 7 " long and about $1 / 4$ " in diameter. The new flow straightener consists of polycarbonate honeycomb and four stainless steel screens. The first two screens are 18-mesh 
screens with the 1 " thick by $1 / 8$ " diameter honeycomb in between. After the second screen is a gap of 1.5 " followed by a 20 -mesh screen, followed by another gap of 1.5 " followed by a 22 -mesh screen. For a complete description of the inlet remanufacture, see the Appendices of Greg Altmann's paper. ${ }^{13}$

Following the completion of the new flow straightener, the flow quality of the wind tunnel was measured. ${ }^{14}$ The project was a senior project by three students. Unfortunately, the quality of the test equipment, calibration procedures, and data reporting has led this author to question the results. Despite these objections, the reported freestream turbulence intensity was measured to be below $0.5 \%$ for most of the test section area, but there was one area, that covers about $15 \%$ of the tunnel, with noticeably higher turbulence intensity and a peak turbulence intensity of $2.7 \%$. The freestream velocity was measured and found to vary considerably, with multiple areas over $2 \%$ and a peak of $3.1 \%$. The turbulence intensity and velocity variation measurements were completed with one wire of a twowire probe (X-probe) connected to a constant temperature system. The probe and measurement system was calibrated in the wind tunnel because the calibration obtained from using the calibrator was not resulting in correct values once the probes were moved from the calibrator to the tunnel. Therefore the object under test was used to calibrate the probe and uncorrected pitot-static probe measurements far upstream of the hot-wire probe were used as the velocity measurement standard.

\subsection{NEW GOLDSCHMIED PROPULSOR MODEL}

The reports from the original Goldschmied wind tunnel tests are either not available or lack some of the details necessary to reproduce their results. The following sections outline the design of the New Goldschmied Propulsor model, here after referred to as the NGP. It will also highlight some of the differences between the previous Goldschmied propulsor model and the NGP, and will outline some of the challenges that arose in the design and manufacturing processes.

\subsubsection{MOdel EXTERnAL SHAPE}

Descriptions of the previous wind tunnel tests do not document the shape of the as-tested body very well. This made replication of this difficult. The best data available to discern the body shape are the locations of the surface pressure ports given in several of the papers. 
The first problem encountered with the surface pressure port locations was that different papers gave slightly different body shapes. One of the differences between the body shapes was the aftbody. The early tests had a pointed aftbody while the latter tests had an aftbody that was open to allow the suction flow to exit through it. Even the two data sets with an open aftbody from the 1982 test differ severely in their aftbody geometry. All data was given non-dimensionalized by the body length. This raw data can be seen in Fig. 3-3. If this data is nondimensionalized by the forbody length, the forbody and aftbody shapes become much more alike. One thing that is clear from Fig. 3-4 is that the aftbody length is the main feature that is different between the data sets. At the time of model design, the test reports contained some pictures that appeared as if the longer aftbody was used and some appear as if the shorter aftbody was used. It was not until late in the manufacturing phase that another test report 5 test was obtained and it showed clearly that both were used. The short aftbody was used by itself, and the long aftbody was used with the cruciform tail.

The longer aftbody was used for the NGP model for the following reasons. The longer aftbody would allow an easier ability to change the exit diameter of the model in future tests to determine the effects that it would have on the model thrust and drag parameters. The longer aftbody would also allow the suction flow to turn more and exit at less of an angle to the longitudinal axis of the model. This straighter flow exiting the aftbody would allow less of a side force issue if the flow exiting the aftbody were not completely axisymmetric.

The second problem with the point data set was the surface quality. The method of modeling these points began by importing them into a CAD program (in this case SolidWorks Student Edition), fit a spline through them to create a curve, and then revolve the curve around the centerline axis. This produced a noticeably wavy surface when viewed from different perspectives. It was determined that the waves were the result of the spline through the imported points. When this spline was revolved, the waves in the spline became rings of waviness, as can be seen in Fig. 3-5, shown as black rings produced from the original point data against the smoothed grey surface. 


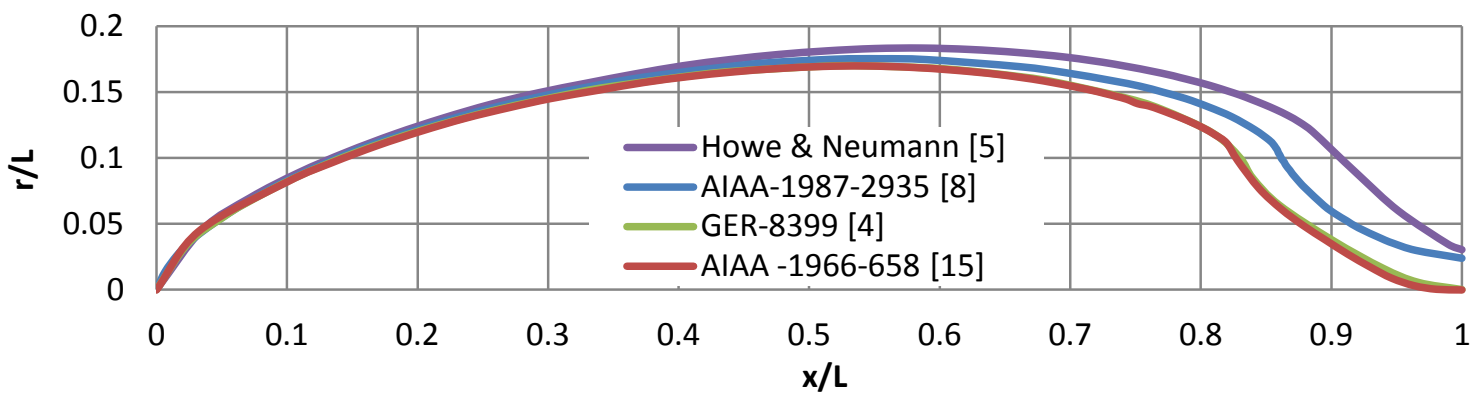

Figure 3-3. Comparison of surface profiles from various sources. All data is non-dimensionalized by the body length. $5,8,4,15$

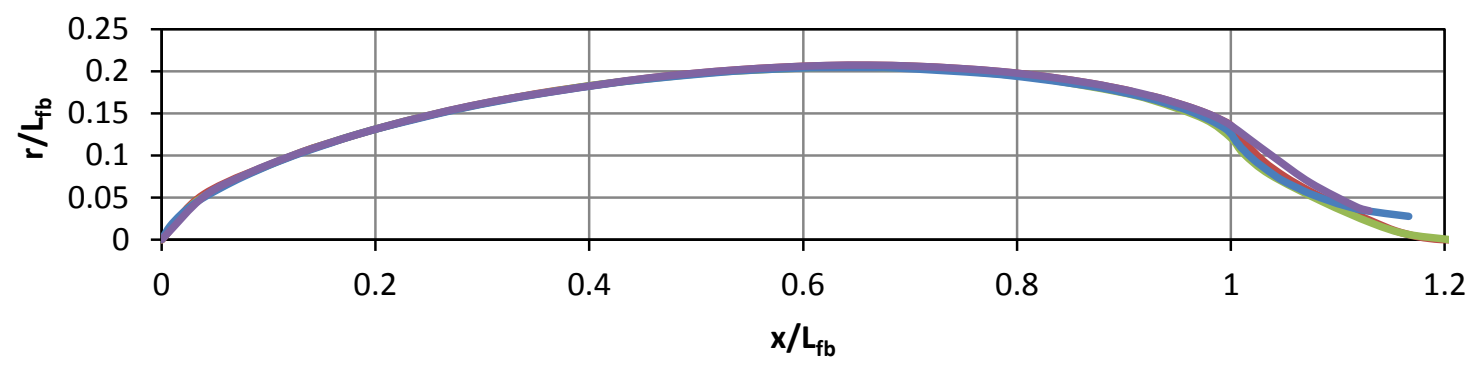

Figure 3-4. Comparison of same surface profiles from Fig. 3-3. All data is non-dimensionalized by the length of the forbody. $5,8,4,15$

Analysis of these points using a 2-D boundary layer code, called PABLO, confirmed the visual diagnosis of the wavy surface. This can be seen in terms of the boundary layer shape factor in Fig. 3-6. This wavy surface was solved by removing some of the points prior to importing them into the CAD program or boundary layer code. Since the points were the locations of pressure ports on the surface of the original models, they were clustered near the nose and the slot. Removing points in these areas resulted in fewer oscillations in the boundary layer shape factor. Originally, the data set contained 57 points. This was reduced to 30 points for the final smoothed surface. 


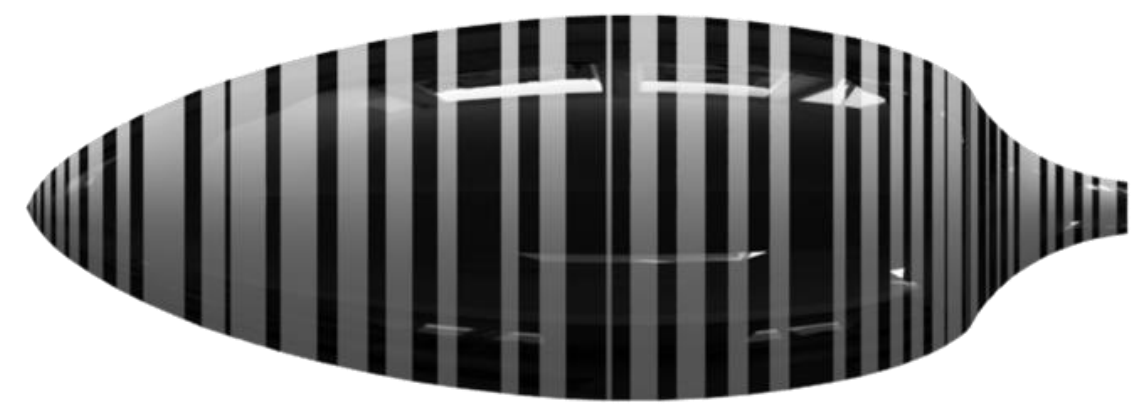

Figure 3-5. Comparison of the smoothed (grey) and unsmoothed (black) model surfaces.

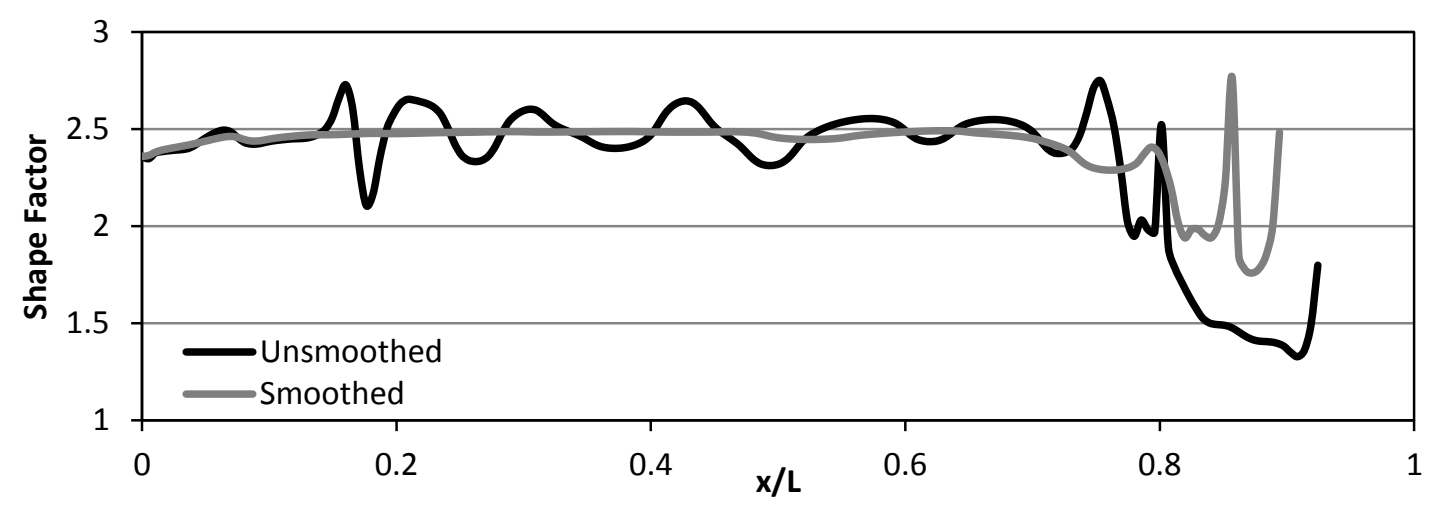

Figure 3-6. Comparison of the bounradry layer shape factor for the smoothed and unsmoothed surfaces.

The third problem with using the pressure port location data to define the model surface is the lack of knowledge about the distance between these pressure port locations and important features of the body. For example, the distance between the last pressure port on the forbody and suction slot is not given. In addition, no information is available about important model features such as the radii, or lack thereof, at the nose, slot entrance, or inside the suction slot. This information was estimated based on figures, diagrams, and plots given in various reports.

\subsubsection{MODEL SIZING}

The size of the model to be tested in the tunnel is an engineering tradeoff. On one side of the tradeoff is the need to minimize the influence of the walls on the measured parameters of the test. To do this, the model must be far from the walls, which dictates the model must be small in dimension compared to the size of the tunnel. On the other side of the tradeoff is the general need to test at a particular Reynolds number to ensure dynamic similitude to the 
full-scale aircraft under test. For the current effort, there is not a full-scale aircraft that dictates the test Reynolds number, but there is the need to compare to previous tests' data. The smallest Reynolds number from the 1956 test was about 4 million, and from the 1987 test, it was 3.2 million. Therefore, 3.2 million was set as the target Reynolds number for this test.

Once the tunnel speed is at its maximum limit, or if the flow is compressible, then at its design Mach number, the only other variable that the test designer has to adjust is the model reference dimension, which would scale the model appropriately. Therefore, to test at a large Reynolds number, the reference dimension must be grown to achieve this desired Reynolds number.

Also driving the model size larger are the sensors, equipment, and instruments that are used inside of it. These items generally cannot be scaled to fit inside a smaller model. For the NGP, the fan unit was very important. A larger model would allow a larger fan. This, in turn, would allow a larger quantity of available power to overcome any suction flow problems that might arise. These fans are available and would probably perform better than the current fan unit. Conversely, if a smaller fan were required, there is not one available with even similar performance to a scaled down version of the current fan.

The solid blockage of the model was the main consideration when sizing the model. Solid blockage has to do with the size of the model in relation to the size of the test section that it is placed in. The model physically blocks the flow inside the wind tunnel. In free air conditions, the air would normally expand around the model, but the walls of the tunnel restrict this expansion. This causes the flow to accelerate as it squeezes between the model and the wind tunnel walls. Therefore, the model experiences a higher velocity than the measured freestream speed. Solid blockage is largely determined by the area ratio of the maximum cross section of the model to the effective cross section of the test section. The actual equation used for this is Eq. 4.2, and will be discussed further later.

The model was sized so that it would experience less than a 5\% change in velocity due to solid blockage. The plot of model size and change in tunnel velocity can be seen in Fig. 3-7. To have less than a 5\% change in velocity with a small amount of margin, the model needed to have a maximum cross sectional area of $9 \%$ of the tunnel cross sectional area. It was recently noted that in the 1956 test, the maximum wind tunnel dynamic pressure correction was about $4 \%$. This gives good credibility to the selected allowable velocity increment. The $9 \%$ model 
resulted in the characteristics that are given in Table 3-1. For comparison, the equivalent parameters from the original Goldschmied tests are included.

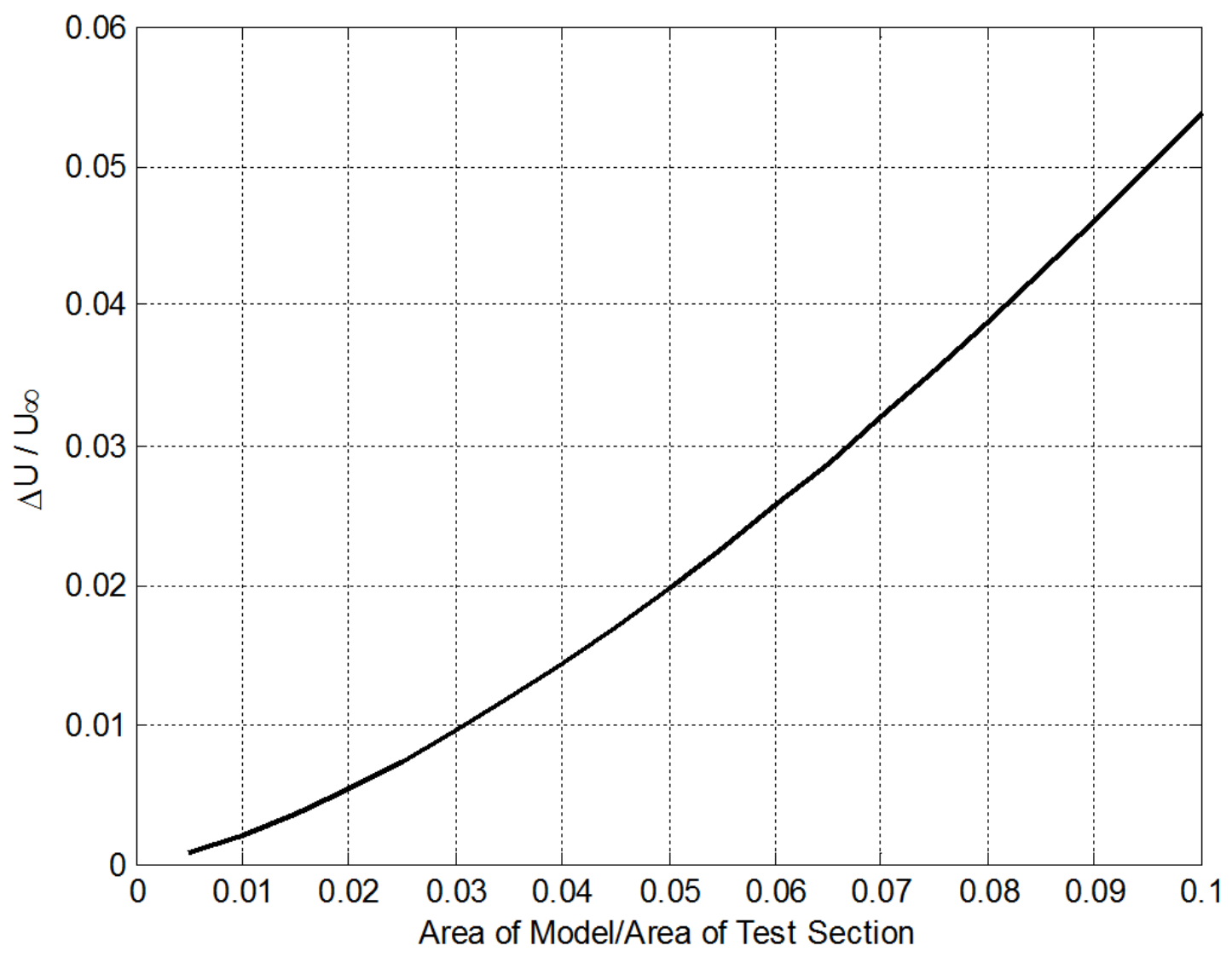

Figure 3-7. The percent increase in the freestream speed seen by the model as a function of the model size compared to the wind tunnel test section size. 
Table 3-1. Summary of the geometric parameters of the NGP and previous Goldschmied models.

\begin{tabular}{|c|c|c|c|c|}
\hline & \multirow{2}{*}{1956 Model } & \multicolumn{2}{|c|}{1982 Model } & \multirow{2}{*}{ NGP Model } \\
\hline & & Short & Long & \\
\hline Reynolds numbers & $4 \times 10^{6}-12 \times 10^{6}$ & \multicolumn{2}{|c|}{$3.2 \times 10^{6}-5.0 \times 10^{6}$} & $1.34 \times 10^{6}$ \\
\hline Tunnel Size & $7^{\prime} \times 10^{\prime}$ & \multicolumn{2}{|c|}{$8^{\prime} \times 10^{\prime}$} & $3^{\prime} \times 4^{\prime}$ \\
\hline Length & $58.8 "$ & $54.45 "$ & $57.17^{\prime \prime}$ & $38.5 ”$ \\
\hline Maximum Diameter & $20.0 ”$ & \multicolumn{2}{|c|}{$20.0 "$} & $13.5^{\prime \prime}$ \\
\hline Location of Maximum Diameter & $54.1 \%$ & $57.9 \%$ & $55.1 \%$ & $55.1 \%(21.23 "$ from LE) \\
\hline Forbody Diameter at Slot & $11.5^{\prime \prime}$ & \multicolumn{2}{|c|}{$11.5^{\prime \prime}$} & $7.75^{\prime \prime}$ \\
\hline Aftbody Diameter at Slot & $9.62 ”$ & \multicolumn{2}{|c|}{$9.35 "$} & $7.00^{\prime \prime}$ \\
\hline Exit Diameter & N/A & $3.3 "$ & N/A & $1.82 ”$ \\
\hline Exit Area & N/A & $8.55 \mathrm{in}^{2}$ & N/A & $2.60 \mathrm{in}^{2}$ \\
\hline Stated Location of the Slot & $86 \%$ & $90 \%$ & $85.8 \%$ & $86 \%$ (33.12" from LE) \\
\hline Body Volume & $6.35 \mathrm{ft}^{3}$ & \multicolumn{2}{|c|}{$6.35 \mathrm{ft}^{3}$} & $1.89 \mathrm{ft}^{3}$ \\
\hline Body Volume ${ }^{(2 / 3)}$ & $3.43 \mathrm{ft}^{2}$ & \multicolumn{2}{|c|}{$3.43 \mathrm{ft}^{2}$} & $1.53 \mathrm{ft}^{2}$ \\
\hline Body Volume $^{(1 / 3)}$ & $1.85 \mathrm{ft}$ & \multicolumn{2}{|c|}{$1.85 \mathrm{ft}$} & $1.23 \mathrm{ft}$ \\
\hline Wetted Area & $17.9 \mathrm{ft}^{2}$ & \multicolumn{2}{|c|}{$17.9 \mathrm{ft}^{2}$} & $8.15 \mathrm{ft}^{2}$ \\
\hline Frontal Area & $2.18 \mathrm{ft}^{2}$ & \multicolumn{2}{|c|}{$2.18 \mathrm{ft}^{2}$} & $0.994 \mathrm{ft}^{2}$ \\
\hline
\end{tabular}

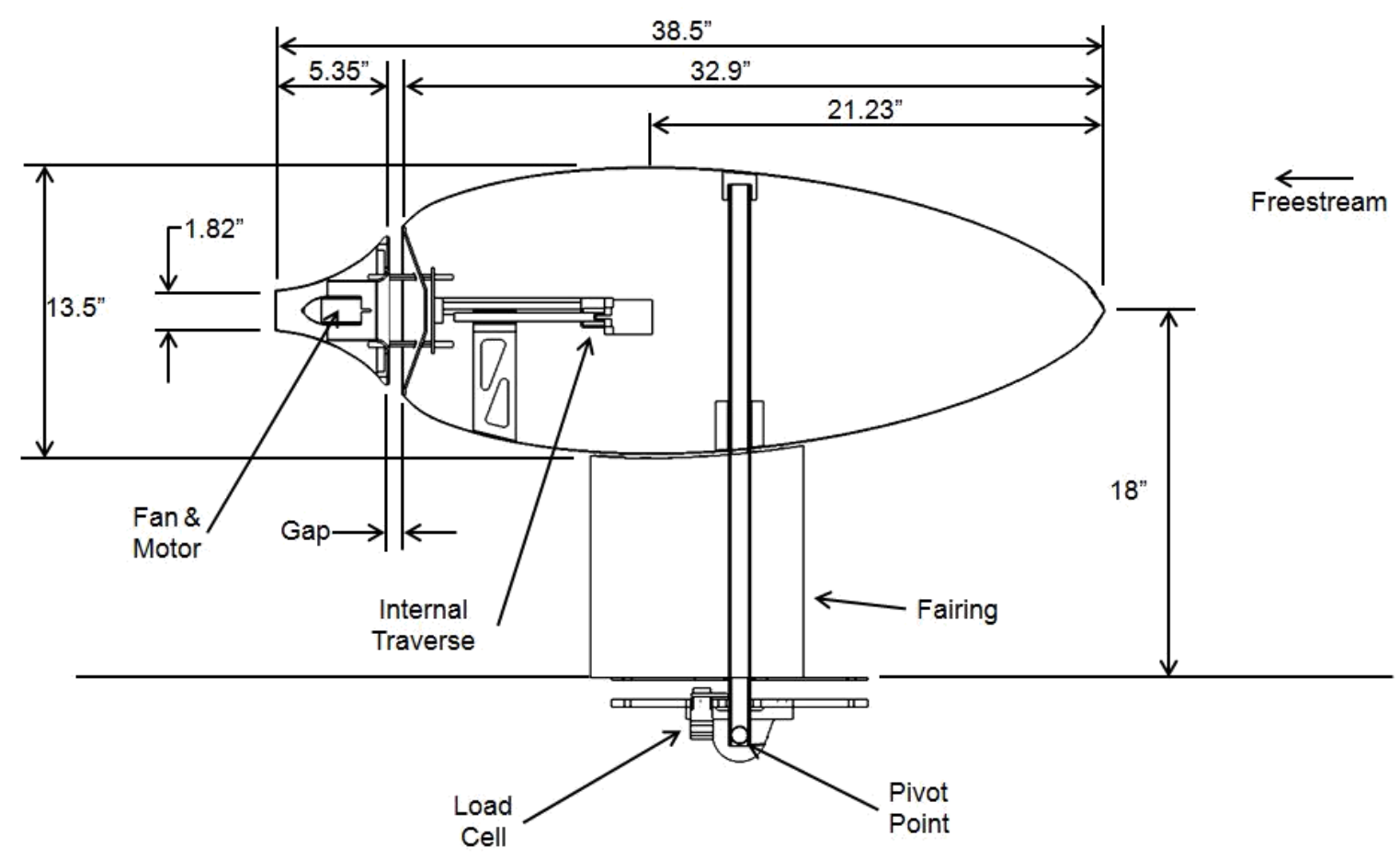

Figure 3-8. General profile layout and dimensions of the NGP. 


\subsubsection{Propulsor}

\subsubsection{Fan}

One of the most important parts of this model was the fan. Without a fan that could provide the suction mass flow rate required the test would suffer from the inability to attach the flow on the aftbody. Input from professors and industry professionals indicated that a fan from the RC airplane industry would have the best chance of working well in the NGP. In the RC aircraft industry, fans are referred to as EDFs, or Electric Ducted Fans. The selection process was difficult due to the lack of data from the fan manufacturers. Typically, fans are accompanied by what is referred to as a fan performance curve, a plot of the flow rate versus pressure rise across the fan for maximum power input. The larger the pressure increase, usually the lower the flow rate and vice versa. A typical fan performance curve can be seen in Fig. 3-9. This curve is from an electronics-cooling fan that was one of the first fans examined as a possibility prior to selecting the EDF. It represents the maximum performance that this fan could deliver continuously. Notice the shaded region below the curve where the fan would operate at less than full power.

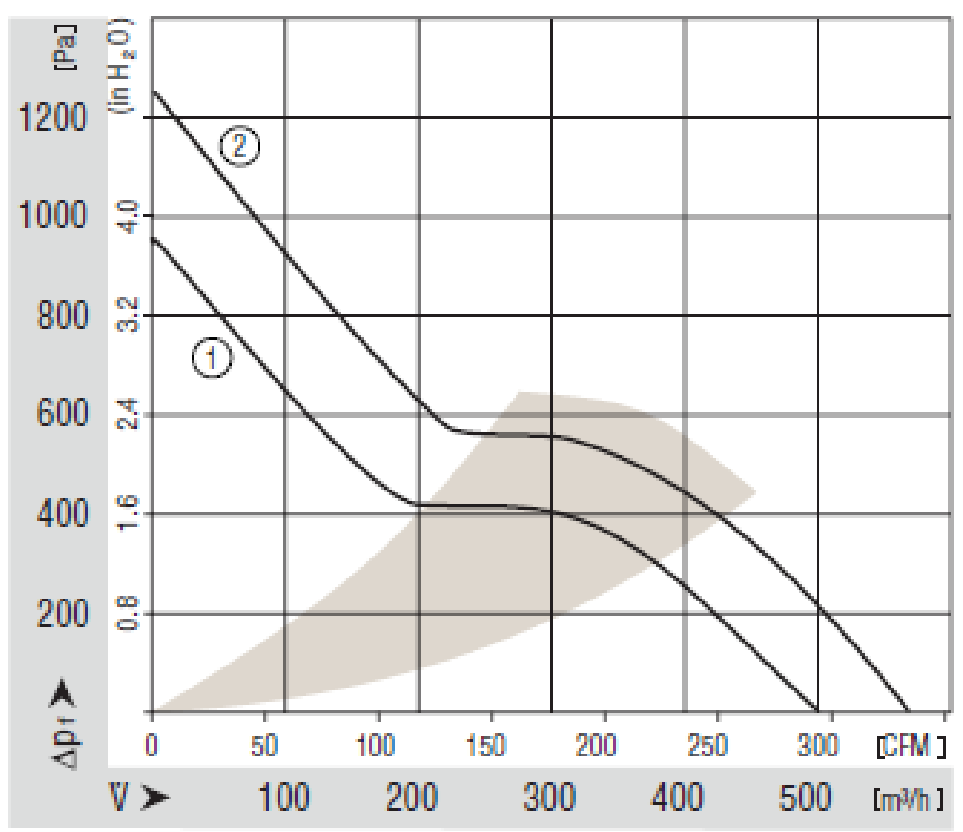

Figure 3-9. The fan performance curve (curve \#2) for a fan considered for use in the NGP. This plot is for a model 4114N/2H8P fan from Ebmpapst Inc. ${ }^{16}$

This type of data was not available for any RC EDFs on the market. One company was found that did provide some basic data that was in a different form from the typical fan performance curve. It provided the fan 
thrust, current, RPM, exit velocity, and efficiency versus the root mean square of the AC voltage supplied to the motor. This information is shown in Figure 3-11. The manufacturer of this EDF was a German company by the name of Schubeler Fans Inc. From evaluating the different fans available from this manufacturer, only two fans that they manufacture would fit inside the NGP. These were 2.84 " $(72.1 \mathrm{~mm})$ and 2.68 " $(68.1 \mathrm{~mm})$ in diameter, and have fan swept areas of $4.03 \mathrm{in}^{2}\left(26 \mathrm{~cm}^{2}\right)$ and $4.65 \mathrm{in}^{2}\left(30 \mathrm{~cm}^{2}\right)$, respectively. Of these two, the $4.65 \mathrm{in}^{2}$ fan had a distinct performance increase over the $4.03 \mathrm{in}^{2}$ fan. Therefore, the $4.65 \mathrm{in}^{2}$ fan was selected with the exact model number being DS-30-DIA HDT. This fan and motor can be seen in Fig. 3-10.

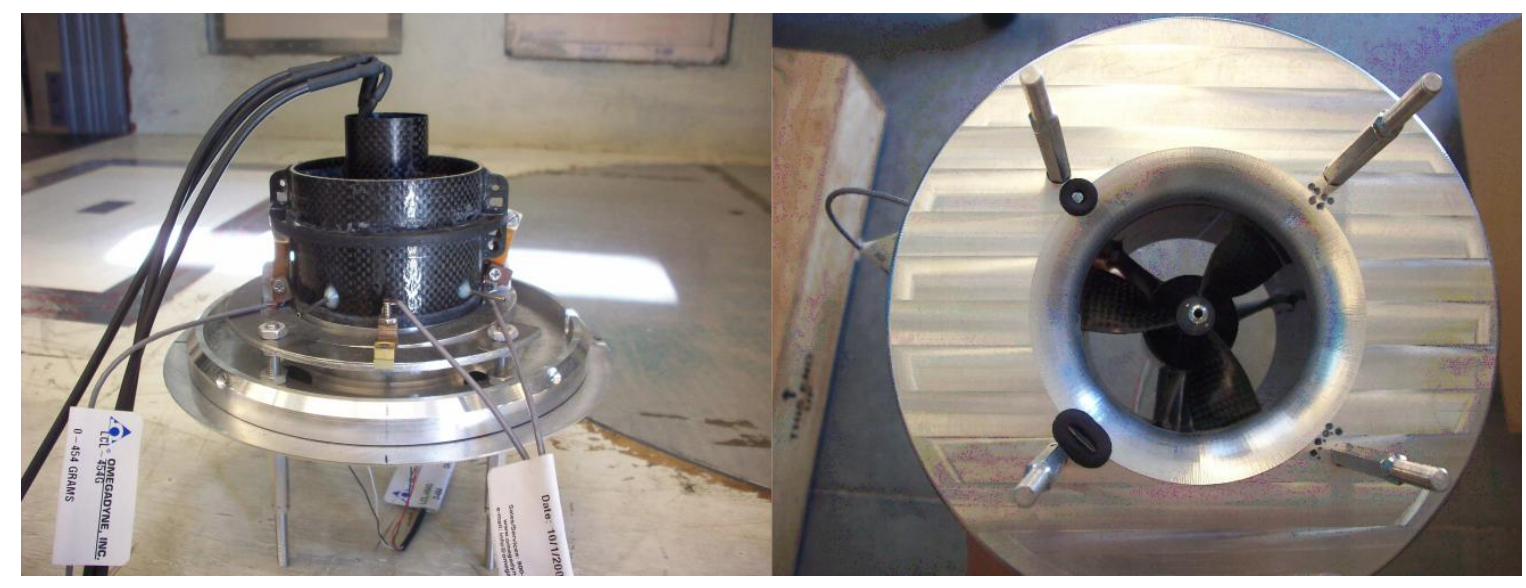

Figure 3-10. Schubeler DS-30-DIA HDT fan and motor (black carbon fiber) preliminarily installed in the aluminum aftbody structure during fabrication.

\subsubsection{Motor}

Motor selection was dictated by the fan selection. Only certain motors are matched to the characteristics of the fan. The RPM range, power output, size, and mounting are the four parameters that must match the chosen fan for safe and reliable operation. In this case, the fan manufacturer had already selected five motors that will work with the DS30. Of those five, only two were available in the United States with less than a 6 weeks lead-time. Of these two motors, one was rated at $500 \mathrm{~W}$ and the other at $700 \mathrm{~W}$. The $700 \mathrm{~W}$ motor was about 0.15 " longer. When both of these motors were placed in the CAD model of the NGP, it was evident that this extra length could interfere more with the exit flow from the fan, so the shorter motor was initially ordered. After initial testing, the 700W motor was also ordered in case the 500W motor could not provide enough power to provide the suction flow required to keep the flow attached on the aft body of the model. The manufacturer of the motor is Hacker Motor Brushless, and the model numbers of the motors are B40-13S, for the 500W motor, and B40-11L for the 700W motor. 
The characteristics of both motors can be seen in Table 3-2, and data from Schubeler with the $700 \mathrm{~W}$ motor and fan combination is given Fig. 3-11. One interesting note is that the peak power in this figure is about $800 \mathrm{~W}$.

Other noteworthy characteristics of the fan and motor assembly are the peak rotational speed of 45,000 rpm, with $50,000 \mathrm{rpm}$ cited by the fan manufacturer as being the safe limit, and peak efficiency of around $65 \%$.

Table 3-2. Summary of the motor properties for the two motors used in testing of the NGP.

\begin{tabular}{|c|c|c|}
\hline & B40-13S & B40-11L \\
\hline Peak Power (watts) & 500 & 700 \\
\hline kV (RPM/Vrms) & 3277 & 2727 \\
\hline Diameter (in.) & 1.09 & 1.09 \\
\hline Length (in.) & 1.85 & 1.96 \\
\hline Weight (oz.) & 4.39 & 5.54 \\
\hline Idle Current (A) & 1.26 & 1.32 \\
\hline Resistance (ohms) & 0.0247 & 0.0212 \\
\hline Poles & 2 & 2 \\
\hline Input Voltage & 3S-4S (12.6V - 16.8V) & $4 \mathrm{~S}-5 \mathrm{~S}(16.8 \mathrm{~V}-21.0 \mathrm{~V})$ \\
\hline
\end{tabular}

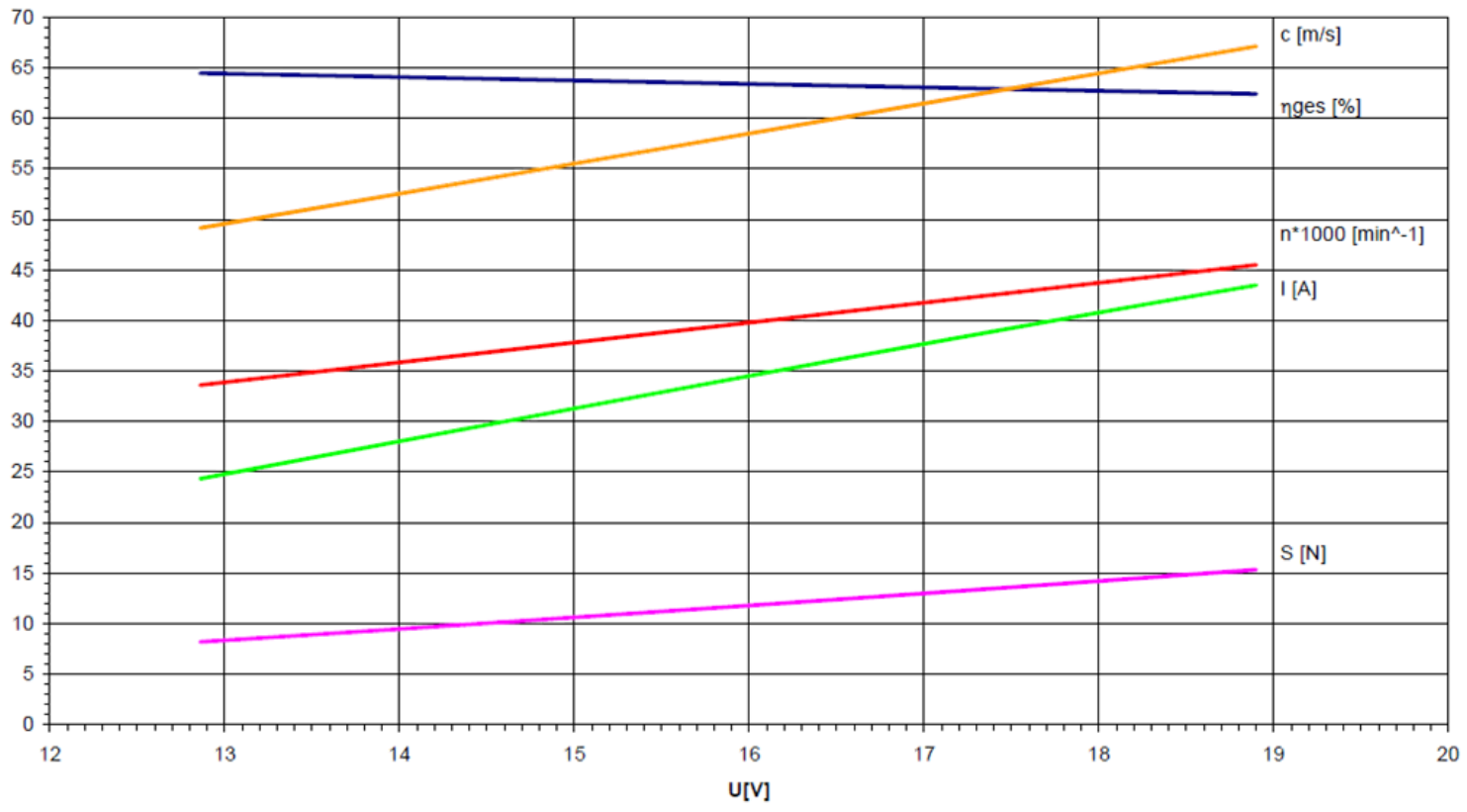

Figure 3-11. Data supplied by the fan manufacturer for the fan and 700W motor together. ${ }^{17}$ 


\subsubsection{Fan Power Supply}

When used in an RC aircraft, the fan and motor are powered by lithium polymer (Li-Po) batteries. These batteries usually last for only a few minutes of constant use before requiring a recharge. To eliminate this problem, as well as the influence of varying battery voltage on fan performance, a DC power supply was used instead of batteries. An HP-6012B power supply was borrowed from the Design-Build-Fly (DBF) Club on campus. The current-voltage characteristics of this powerful power supply can be seen in Fig. 3-12. To simulate the Li-Po batteries, the peak cell voltage of $4.2 \mathrm{~V} /$ cell was duplicated on the power supply. In the RC industry, the cells are placed in series to increase the voltage to the motor. If four cells are in series, this is known as a $4 \mathrm{~S}$ battery. The 500W Hacker motor is designed to operate at either $3 \mathrm{~S}$ or $4 \mathrm{~S}$, while the $700 \mathrm{~W}$ motor operates at either $4 \mathrm{~S}$ or $5 \mathrm{~S}$.

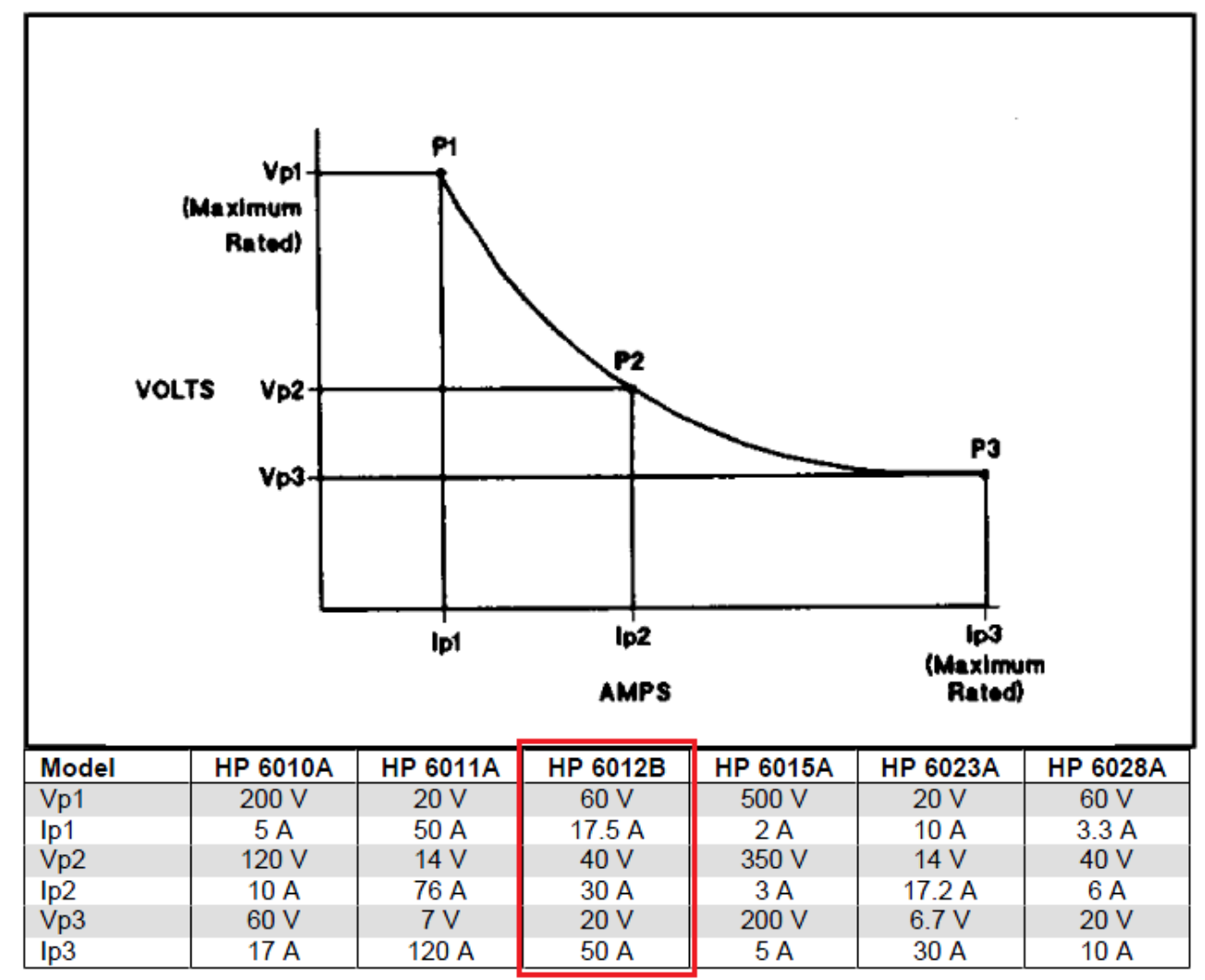

Figure 3-12. Voltage \& Current characteristics of the HP6012B power supply used to power the EDF during all testing of the NGC. ${ }^{18}$

\subsubsection{Tunnel Mounting Location}

The initial design of the NGP was to mount the model to the sting balance in the wind tunnel and utilize the sting balance to measure the forces on the model. This was eliminated due to three factors, the first of which 
was that the sting is very close to the end of the test section. At the end of the test section is the diffuser, and at the end of the diffuser is the wind tunnel fan. There was some concern that the proximity of the model to the diffuser and wind tunnel fan would cause some unknown aerodynamic effects since the longitudinal pressure gradient is not documented in this area of the tunnel.

The second is that the planned wake survey would have had to be done at about one body diameter (about $1 / 3$ of the body length) downstream from the model due to the close proximity of the model to the diffuser. This close to the model, the survey probe would have still been in the separated and disordered wake of the model. A wake survey is traditionally done with a series of total pressure probes far enough downstream of the model where the wake has returned to a uniform cross-sectional static pressure and any transverse velocity has been appreciably damped out. When completing a wake survey in this manner, the total pressure probes read the true velocity profile. In this current case, a traditional pitot static probe would not have read the true velocity profile in the wake as required for an accurate calculation of the net axial force. A multi-hole probe would need to be used to capture the true velocity profile this close to the model. A seven-hole cobra probe is available in the wind tunnel lab, but it has not been used in any previous experiments. Its use was researched and determined to require a considerable effort. This level of effort was avoided due to the considerable effort already undertaken for the design, manufacture, and testing of the NGP.

The third reason mounting to the sting balance was abandoned was that the load limits of the sting balance are very small compared to the expected forces from the NGP. The maximum pitching moment of 150 in-lbs became the limiting factor of the model's weight. Because of this limit, the model would have needed to be much lighter and smaller. This smaller model would have been beneficial in terms of blockage effects, but fan selection, wire routing, tube routing, and sensor mounting would have been considerably more difficult.

The above-mentioned reasons required that the model be mounted in another location in the tunnel. This requirement necessitated the design and manufacture of a strut to hold the model in an existing model mount location. Besides the permanent test section that houses the sting balance, three removable test sections could be used to mount the model in. Two of these sections are five feet in length and contain three removable panels on each of the four walls. If one of these 5' test sections were to be used, a new panel would have had to be created to mount the model strut to and a second panel would have had to be created to mount the traverse to in order to 
complete the planned wake survey. The length of each test section necessitated the use of both of these test sections in combination. The third test section is an 8 ' long new test section that has a slot in one side to allow probes and survey equipment through. This test section was chosen because of this built-in functionality. It was used without either of the other two test sections in combination to prevent the wall boundary layers from growing too large prior to the model location. This was a concern because a previous test discovered that the floor boundary layer grew about 4 inches (400\%) in the 5' length of a short test section. This growth of the wall boundary layers would reduce the test section area where there is freestream flow by about $30 \%$. Therefore, to allow the greatest possible effective tunnel area, the short test section was removed. The only available location to mount the model in this test section was close to the front of the test section. This resulted in the model nose being almost coincident with the exit of the contraction. This was deemed undesirable, but acceptable.

\subsubsection{Mounting Strut ANd FAIRING AsSEMbLy}

During the initial stages of the model design, the availability of funding was limited. Therefore, a high accuracy load cell that was already present in the wind tunnel lab was selected and the method for measuring the axial force on the model was designed around it. The overall method of measuring the axial force through the strut, mount, and load cell was developed through observing systems that measure the weight of very heavy items with fine precision and accuracy. The defining characteristic of these types of scales is some sort of mechanical advantage. For the present application, the model's small axial force needed to be amplified so that it could be measured accurately by the chosen load cell.

The system designed is a simple lever system. The model acts on the end of a long lever, the strut, which pivots about a point under the tunnel. The load cell is mounted so that it applies a force in the opposite direction as the model when the model has a net negative axial force, and it contacts the lever at a much smaller distance away from the pivot point. The mechanical amplification of the axial force of the model is then the ratio of the geometric lever arms about the pivot point. The load cell has a maximum range of $25 \mathrm{lbs}$. From test data of the 1956 test, the largest measured drag coefficient was 0.002 , or 20 drag counts. This results in a maximum load of $2 \mathrm{lb}$ of negative axial force under the expected test conditions. The mechanical advantage was then set nominally at 10:1 to allow a full-scale reading on the load cell of $20 \mathrm{lbs}$. 
The first design requirement for the strut was that it must have a small cross sectional area to allow for a thin airfoil shaped fairing around it. A thin fairing would have a small blockage effect and the wake would be small as to not influence the aerodynamics of the model. The second requirement was that it needed to be stiff to prevent model deflection under load. The strut was sized originally to have less than 0.010 " of deflection in bending under this calculated maximum load. This deflection would result in about $0.03^{\circ}$ of pitch angle change when the model goes from maximum negative axial force to zero axial force. This was deemed negligible compared to estimated deflections due to tolerances in the rest of the mount and deflection of the load cell itself. The selected raw material is 4130 rectangular steel box with the dimensions of 0.5 " $1.0 " \mathrm{x} 0.065$ ". The cross sectional area was deemed acceptable since it was only 0.5 " in width.

The general arrangement of the model strut and mounting assembly can be seen in Fig. 3-13. The strut is what the test article is mounted to in the tunnel. This strut is welded to a cylindrical bushing that is pressed onto a solid steel shaft. This shaft is then coupled to a set of flexure joints using two aluminum shaft couplers. The flexure joints are then clamped into a set of aluminum shaft supports. This can be seen in Fig. 3-14. The shaft was used to spread the mounting locations apart. This was done, instead of placing the pivot joints directly at the bottom of the strut, to be able to resist any yaw moments from the model. It was believed that if a mount came loose, a weld broke during testing, or even an operator error by not properly aligning the model to the flow, that by widening the mounting locations of the pivot points, that they would more effectively withstand these forces and moments. It also would prevent the tendency of a small error during the manufacturing of the mounting points to cause a large misalignment of the model when everything was assembled. 


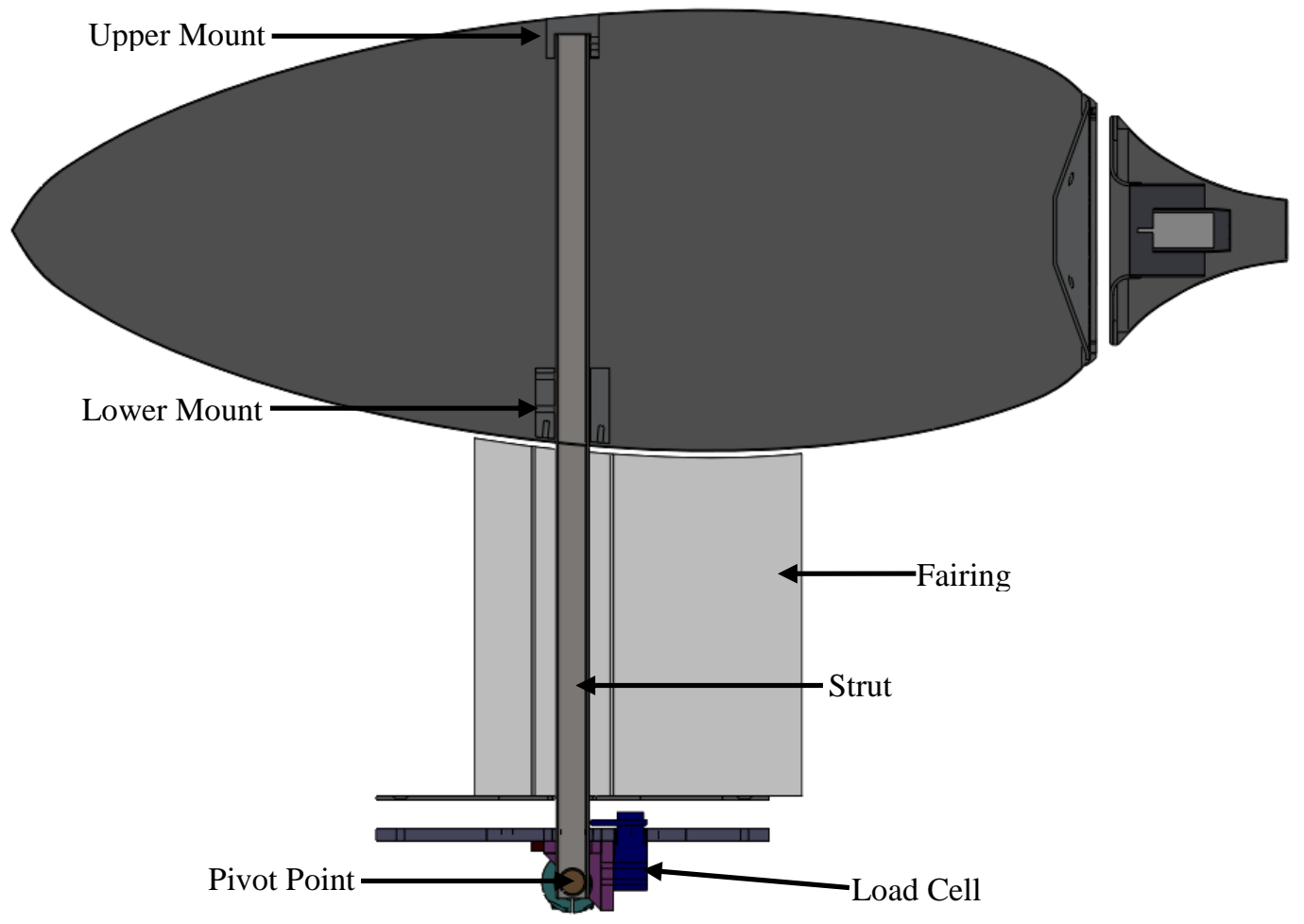

Figure 3-13. General configuration of the model and model mount assembly.

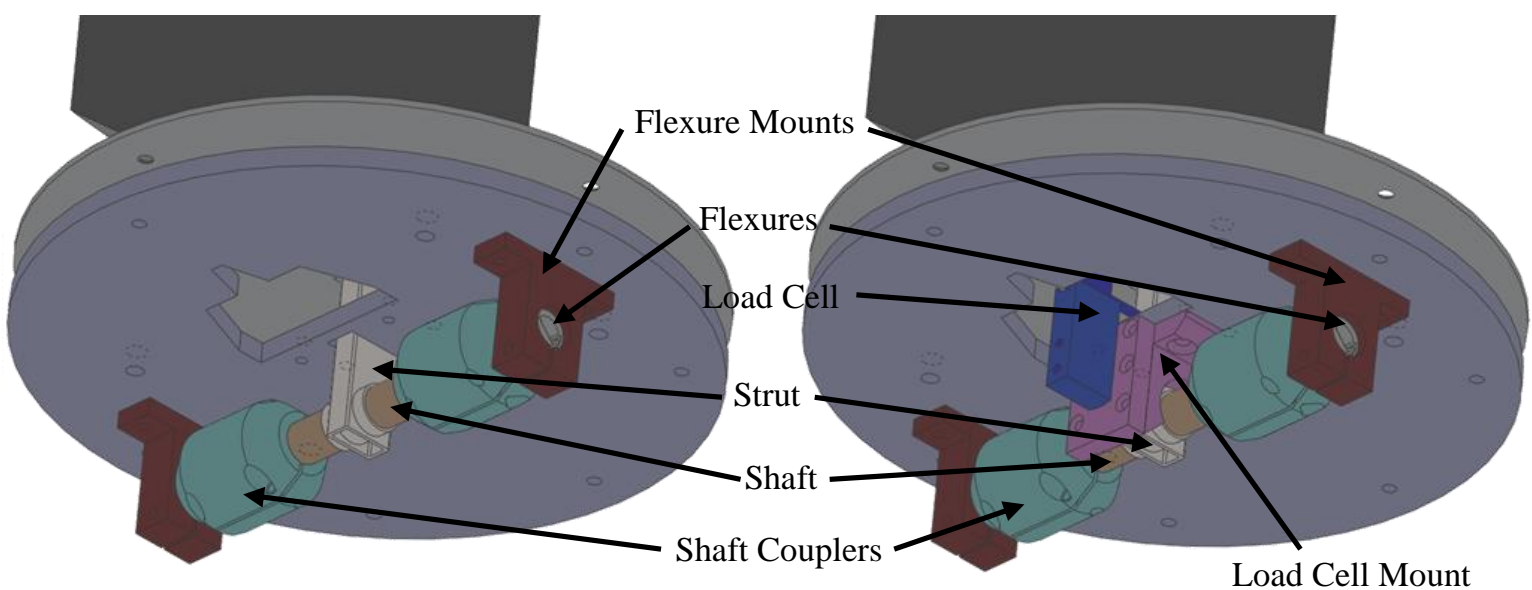

Figure 3-14. Underneither view of the model mounting base, pivots, flexures, and strut. The left picture is without the load cell and load cell mount, while the right view has them included. 


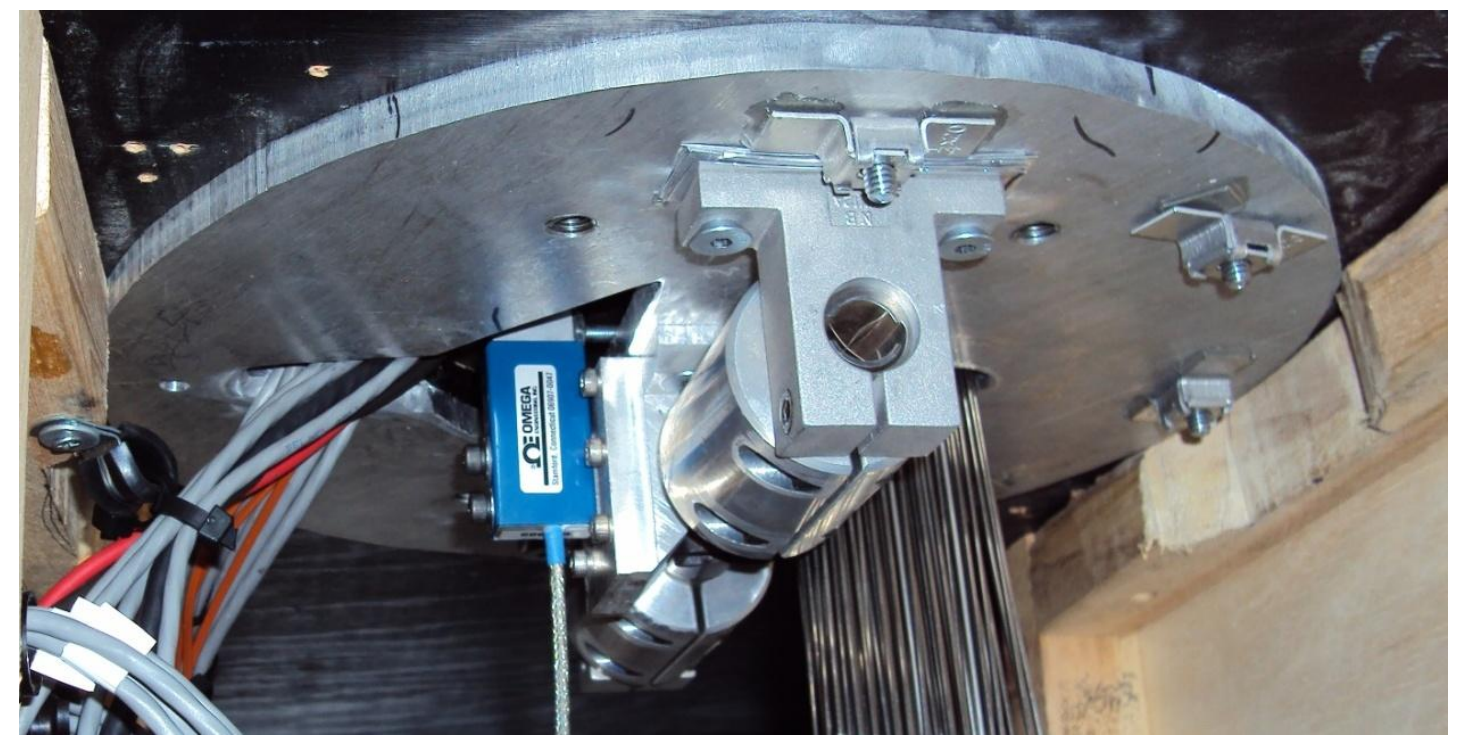

Figure 3-15. Actual model mount with the load cell for comparison with the CAD model.

\subsubsection{Flexure Joints}

The original design and build of the model used bearings, or pillow blocks, to mount the shaft and allow the model to pivot. These proved to have a lot of static friction, or stiction, which was not foreseen. The stiction was evident in the initial attempts at calibration of the axial force load cell. The calibrations were not repeatable and the residuals of the calibration curve were approximately $25 \%$ of the maximum anticipated axial force. Upon expert advice by an experienced engineer, the bearings were replaced with flexure joints. The flexure joints allow the model to pivot, as if connected to bearings, but without the static friction that a bearing has. These flexure joints have zero friction when mounted correctly and operate below their rated capacity. They have a range of motion of $\pm 30^{\circ}$ from their nominal position. When they rotate, the internal vertical and horizontal panels deform. The internal panels are configured and sized to support the design loads. They do however have a nonzero torsional stiffness, or spring rate, due to the bending of the panels. The selected flexures are from C-Flex Bearing Company Inc. They are the model number I-10 and can support up to $30 \mathrm{lbs}$ each. The torsional stiffness of each flexure is stated as $0.0482 \mathrm{in}-\mathrm{lbs} / \mathrm{degree}$. If this stiffness were too high, the load that would otherwise be transferred to the load cell could be used instead to overcome this stiffness, giving the load cell poor resolution. However, with this low of a torsional stiffness and the low deflection of the model during testing, the affect of the flexures on the output of the load cell is deemed immeasurable. The CAD model of the flexures, as well as the actual flexures mounted in the mount assembly can be seen in Fig. 3-16 


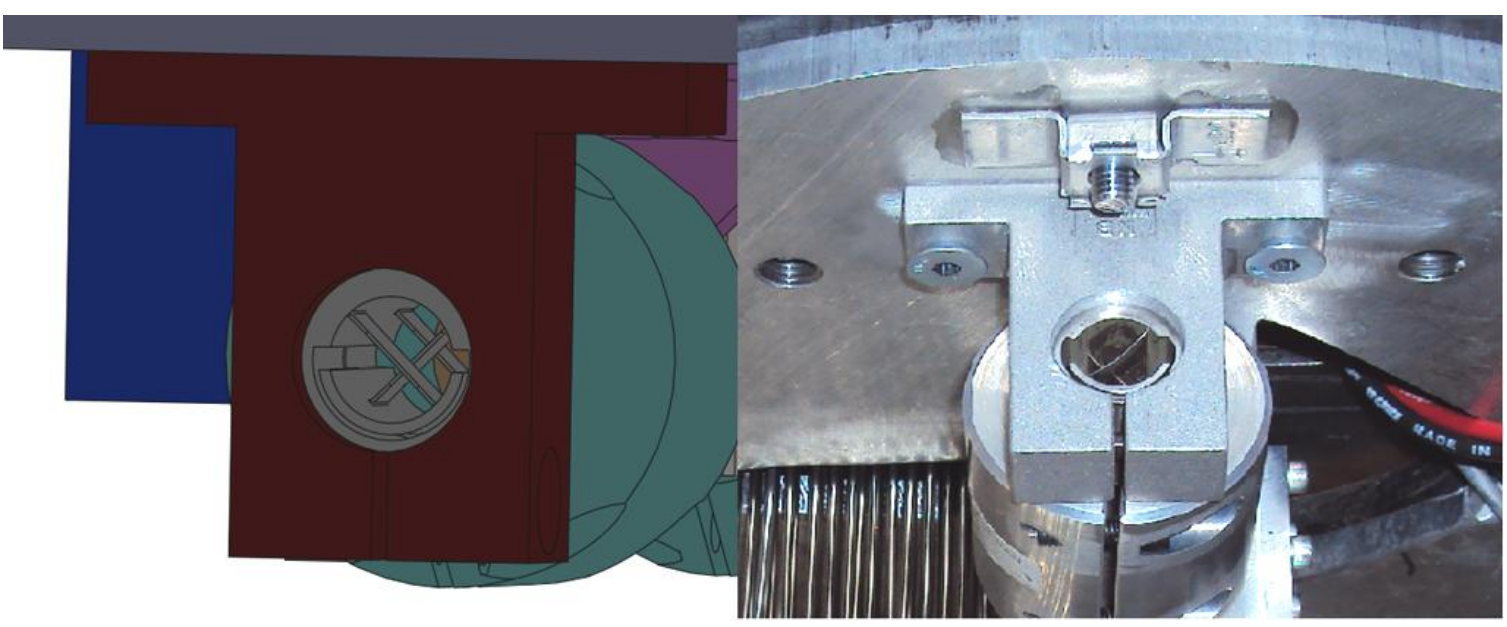

Figure 3-16. Flexure joints were used as the pivots because they allow frictionless rotation.

\subsubsection{Fairing}

In general, a fairing and strut can have many different configurations. The fairing could be attached, or not, to the strut. It could extend the entire distance from the tunnel floor to the model surface, or it could stop a short distance away from the model leaving a short exposed length of the strut. By not extending the fairing all the way to the model, there is less interference of the model's flow field by the fairing because it is usually of small cross section. In addition, these two options allow multiple possible combinations and variations. For the NGP, the fairing has to be extended to the model to shield the wiring and pressure tubing as they run from the model to outside the tunnel because these items could not fit inside the strut. One option was to grow the size of the strut to allow all of these items to fit inside of it, but the resulting strut would have to be very large. Then the flow field influence of this non-aerodynamic strut would probably be larger than the larger, but aerodynamic, fairing. Once this was decided, the decision to attach, or not, the fairing to the strut needed to be made. If it were, then the fairing could also be attached to the model and the joint between the fairing and model could be very smoothly faired to allow the smallest influence on the model flow field by the strut. However, it would also add an additional force to the measured axial force of the model. This force from the fairing would have to be accurately measured and corrected for by measuring, or calculating, the drag of only the fairing and subtracting it form the measured axial force. To do this accurately was judged more difficult than simply fixing the fairing to the tunnel floor and not allowing it to contact the strut. Therefore, the final fairing extends from the floor of the tunnel to the model while not contacting 
the strut or model. There is a small gap of about $1 / 8$ " between the fairing and the model. This gap allows for model adjustment without it contacting the fairing.

For simplicity, a constant chord and thickness airfoil was desired across the entire span. The airfoil thickness and chord need to be large enough to have enough internal space yet short and narrow enough to not appreciably separate near the trailing edge, which could interfere with the BLS. NACA 0012 and NACA 0015 airfoils with chords from 6" - 10" were examined in a 2D boundary layer code called CEBSMITH to examine the amount of separation at the trailing edge. The $15 \%$ thick airfoil showed a noticeable increase in the thickness of the separation at the trailing edge when compared to the $12 \%$ thick airfoil. The selected faring is a NACA 0012 airfoil with a $10 "$ chord. The combination of the thinner profile and the large chord allows for enough internal space without significant separation at the trailing edge.

The fairing is constructed from four wood ribs and four wood spars with a thin plywood covering, which is then cover by Monokote $®$. The plywood, spars, and all but the top rib are a hard wood while the top rib is a thick piece of soft balsa wood. The soft balsa allowed the top rib to be easily contoured to match the contour of the model. The fairing is mounted to the base plate in the wind tunnel. This base plate sits inside a recessed area in the floor of the wind tunnel. The ribs originally had a small cutout for the strut. These cutouts were enlarged to prevent the strut from rubbing on it and to allow a larger range of strut rotation. The latter was required to get the model properly aligned in the wind tunnel.

Through the fairing runs all the wiring and tubing that is connected internally to the model, as can be seen in Fig. 3-17. The wiring is allowed to hang loose inside the faring. It is attached to the model toward the top of the strut inside the model, and outside the tunnel, it is attached to the tunnel. This is a source of friction in the model mount and strut. This friction is a source of hysteresis and non-repeatability in the axial force calibration and actual wind-on measurements as well. The wiring is a necessary part of the model, and its placement is critical to a good model installation. The wiring was allowed to hang loosely through the strut as to try to minimize these effects on the model measurements. To run the tubing through the fairing, it was decided to use a hard line through the fairing, and then connect soft lines to the hard lines both inside the model and underneath the tunnel. The hard line allowed the 60 lines of tubing that run into the model to take up a much smaller space inside the fairing than soft lines. However, connecting the tubing during model installation is a major time commitment. In a professional test 
environment, the Scanivalve would be mounted inside the model. This would result in considerably less time connecting the tubing because it could be done during the model prep and not in the tunnel. However, this is considered risky by some due to the fragile nature of the equipment, large cost for repair or replacement if a mishap were to occur, and the likelihood of a mishap occurring with inexperienced students. Therefore, the timesaving is not warranted and the tubing is run out of the tunnel.

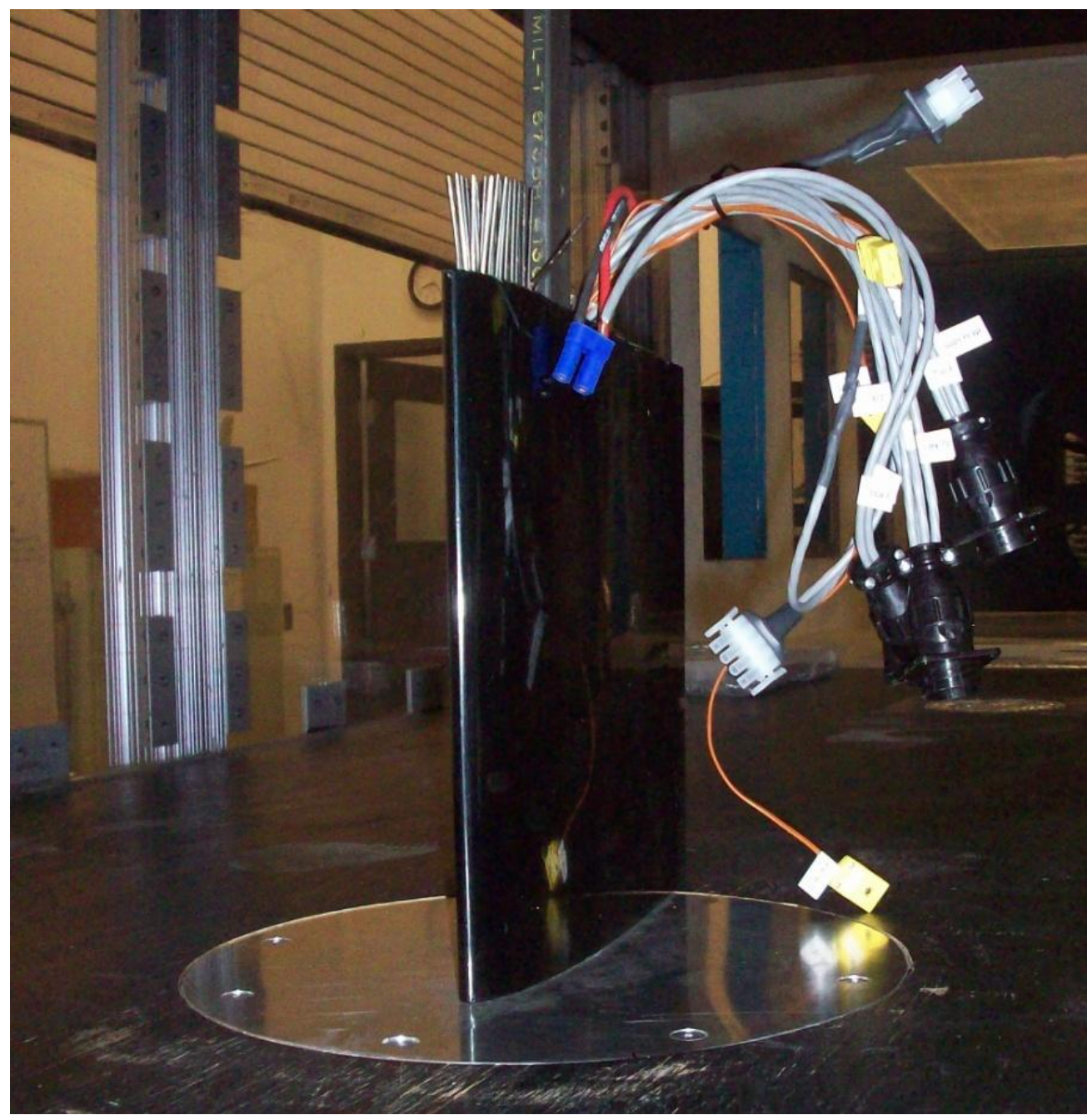

Figure 3-17. The fairing and baseplate mounted in the tunnel with the strut, stainless steel pressure tubes and wiring visible. 


\subsubsection{Strut and Fairing Placement}

The strut was placed in the fairing so that it was centered at the thickest part of the fairing. This was really the only way the strut would fit inside the fairing with enough range of motion in both directions to allow adjustments of the model pitch without also having to account for strut to fairing interference. The model mount assembly was placed in relation to the model so that the fairing was mostly in a favorable pressure gradient area of the model. The favorable pressure gradient on the model extends from the leading edge to the maximum thickness. Seventy percent of the fairing airfoil length is forward of the model maximum thickness. This was done to prevent, and minimize, as much destructive interference as possible between the model and the fairing due to the required gap between the two. The fairing and strut could not be moved too much farther forward without exacerbating CG placement issues that are discussed later in Section 3.2.6.2.

\subsubsection{Model Alignment}

A number of adjustability features are designed into the model mount. The link between the strut and the model are the upper and lower mount blocks. These mounting blocks, seen in Fig. 3-18, attach to the right half of the model, and they are fastened to the strut with multiple setscrews. The setscrews are on two of the four vertical sides of the mounting blocks. The mounting blocks' internal dimensions are $1 / \frac{1}{8}$ " wider than the strut laterally and longitudinally. This extra space in the mounting blocks, along with the setscrews, are to allow the use of shims to precisely align the pitch and roll angles of the model independent of the strut alignment. It allows adjustment of both pitch and yaw, depending on where the shims are placed in the mounting block. This adjust is done during model installation and is the primary adjustment mechanism because it is has the finest resolution, however, it requires that the model be disassembled to make the adjustment. Therefore, a secondary adjustment of the model pitch can be accomplished by adjusting the extension screw on the load cell. This is done as the final adjustment because it does not require model disassembly, and with the right finesse, it can be done just as accurately. The secondary adjust for yaw alignment of the model can be accomplished by loosening and adjusting the entire model and mount assembly. It is a much cruder method of adjusting yaw, but it again can be accomplished accurately with the right finesse.

During model installation and adjustment, the yaw alignment was completed by geometrically aligning the vertical center plane of the model to be less than $0.2^{\circ}(1 / 8$ " difference between nose $\&$ tail) from parallel with the left wall of the tunnel. For pitch, the horizontal center plane of the model was geometrically aligned to be less than $0.1^{\circ}$ 
$\left({ }^{1} / 16\right.$ " difference between nose \& tail) from parallel with the floor of the tunnel. No effort was made to try to align the model with the local flow direction of the tunnel because the amount of up-flow or side-flow in this area of the tunnel is unknown.

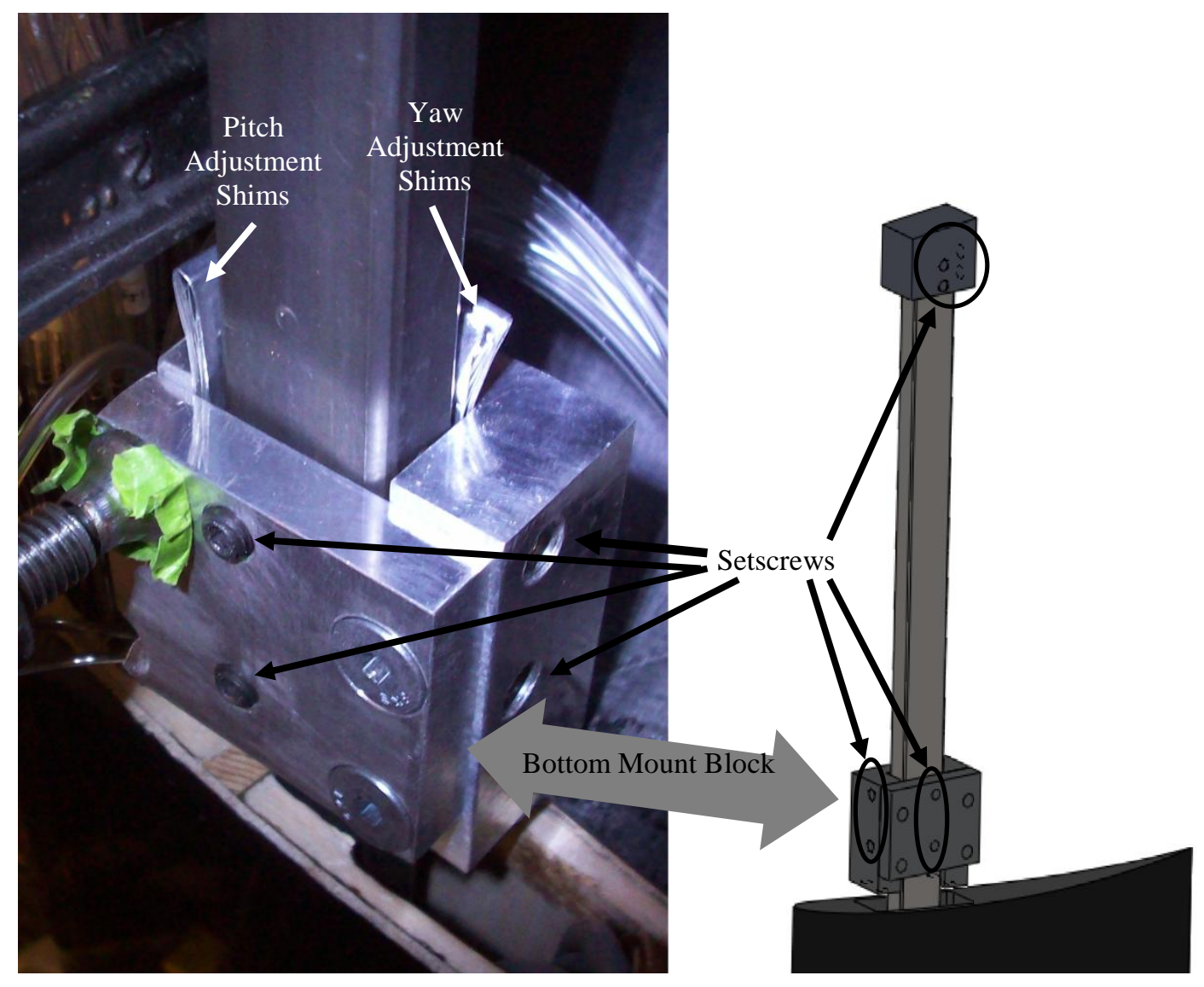

Figure 3-18. Bottom mounting block showing the adjustment set screws and shims used for alignment in the pitch and yaw axis.

\subsubsection{Net AXIAL ForCE MEASUREMENT}

The requirements of measuring axial force in a wind tunnel environment are very difficult requirements to meet. On one hand is the need to measure large loads. On the other hand is the very small resolution required to detect very small and subtle changes made to the models, which results in equally small changes in those large loads. The ratio of the largest load to the smallest resolution can sometimes exceed 75,000 in commercial wind tunnels where resolution requirements for axial force balances are in the range of one drag count. ${ }^{19}$ For the NGP, one drag count would equal $0.1 \mathrm{lbs}$ resolution in the axial force measurement. This was deemed the target for the measurement of the axial force in this experiment. 


\subsubsection{Load Cell Characteristics}

The position of the load cell sensor and overall concept were discussed in Section 3.2.4. The sensor itself is a full Wheatstone bridge load cell. It has a full-scale range of $\pm 25 \mathrm{lbs}$. it has a nominal sensitivity of $3 \mathrm{mV} / \mathrm{V}$. This means that the sensor has an output of $3 \mathrm{mV}$ per $1 \mathrm{~V}$ of excitation voltage at full scale of the sensor. It is excited at nominally $10 \mathrm{Vdc}$. This results in a nominal $\pm 30 \mathrm{mV}$ output from the sensor, or $1.2 \mathrm{mV}$ per pound of force. With the 10:1 mechanical advantage of the model mount, this is nominally $12 \mathrm{mV}$ per pound of force on the model. Even though the sensor had been calibrated at the factory and the actual sensitivity of the sensor was provided with the sensor, it was calibrated with the model mount, wiring, tubing, etc in place to account for any friction and hysteresis present in the system.

Safe overload on the sensor is $150 \%$ of capacity, or $37.5 \mathrm{lbs}$, or $45 \mathrm{mV}$. It is temperature compensated in a temperature range of $0^{\circ} \mathrm{F}-150^{\circ} \mathrm{F}$. This means that the strain gauges have a very similar coefficient of thermal expansion (CTE) as the base material in this temperature range. This results in the output not changing appreciably due to thermal expansion of the sensor in this temperature range. It has a linearity, hysteresis, and repeatability of $0.03 \%, 0.02 \%$, and $0.01 \%$ of full scale, respectively. The stated creep of the sensor is less than $0.15 \%$ of full scale after 20 minutes at full scale. If these errors are simply added together, the resulting maximum expected error is $0.21 \%$ of full scale, or $0.0525 \mathrm{lbs}$. This corresponds to about 0.5 drag counts of accuracy, which meets the desired accuracy level for the current tests from this simplified perspective.

\subsubsection{Axial Force Free Body Diagram}

Previous wind tunnel tests of a Goldschmied propulsor produced both axial forces in the positive and negative direction. Therefore, it was anticipated that the current test would do the same. Therefore, a way of measuring the axial force in both directions was required. Many solutions to this problem were examined in an informal trade study. One of these solutions was to use two load cells, one for each direction. This proposed solution was believed to over-constrain the situation with two load cells pushing on the strut at once. Another proposed solution was to use a solid link between the strut and a single load cell with a load cell that could measure in both directions. This solid link would be in compression for force in once direction and tension for the force in the opposite direction. However, the switch over from positive to negative, or vice versa, would likely be accompanied by pitch changes due to compliance of the load cells and tolerances in the mechanical links. 
The main problem with both these systems is that when the net axial force changes from negative to positive, the force on the load cell will change sign also, and this change in direction of the force on the load cell, is what had the negative effect of pitch change. Therefore, to prevent this, a method that induced an offset in the load cell reading was found. This offset resulted in the load cell still reading force in one direction for axial force in both directions. This was accomplished by not placing the center of gravity, or CG, of the model directly coincident with the model strut. The CG was placed aft of the model strut. This aft placement resulted in a moment about the pivot point of the strut. This moment is what provides the offset in the measurement because even when there is no load on the model, this moment has to be countered by the load cell, which causes a force to be measured. It is not until the model produces enough positive axial force to overcome the moment that the strut will lift off the load cell and pivot forward. As can be seen in the free body diagram in Fig. 3-19, the moment is the product of the model weight and the distance between the model CG and the strut centerline. This allowed the offset to be adjusted during the model setup to allow the right amount of offset.

The right amount of offset was in practice difficult to achieve. If the CG was too far aft, then the load cell could easily exceed its safe overload limit, causing permanent damage to the load cell. If the CG were too far forward, the model and strut would pitch forward and lift off the load cell's contact point with just a small amount of positive axial force. To manage the CG of the model, a bin was added to the front of each model half. This bin held weights to move the model CG forward. In the model's final testing state, there was 5 lbs of lead weight in the nose of the model. This resulted in a CG offset of about $3.5 \mathrm{lbs}$ in the final calibration. This could have been reduced, but it was deemed acceptable since to reduce it, the model had to be disassembled and reassembled.

By examining the FBD, an equation can be derived to relate the force on the load cell to the force from the model. This equation is solved for the force on the model, and it is shown in Eq. 3.1. A consequence of this approach is that the force read on the load cell depends on the CG of the model, but the CG of the model changes when the aftbody is moved to adjust the slot, as shown in Eq. 3.2. This originally was going to result in a separate correction factor depending on location of the slot, but it was soon realized that this effect would be taken into account when the load cell zero shift is taken into account, as will be discussed in Section 4.2.5. 


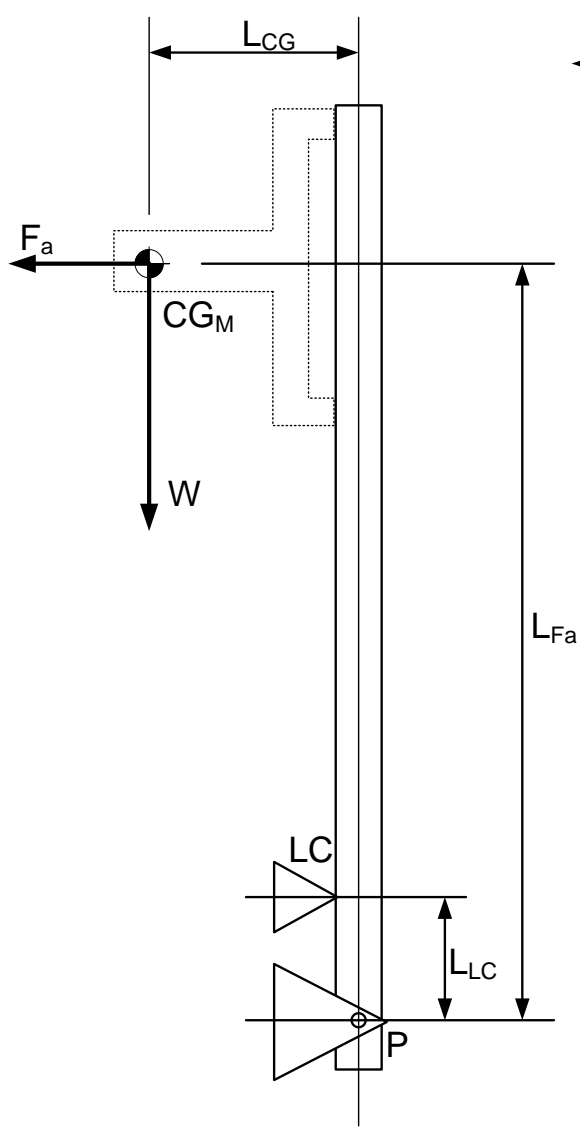

$\bigcup_{\infty}$

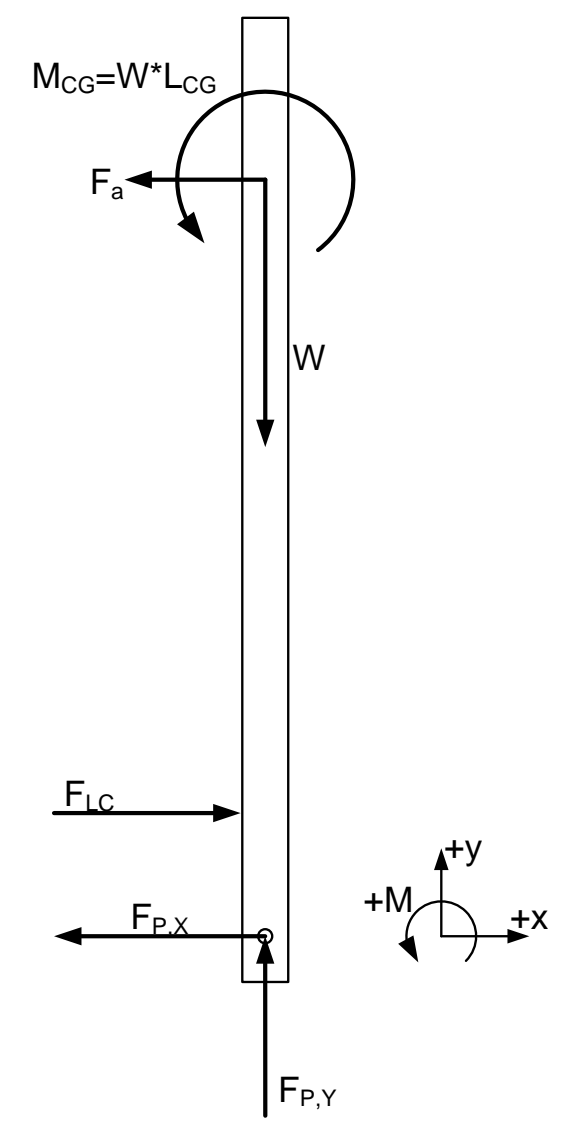

Figure 3-19. Layout diagram (left) and free body diagram (right) of the model strut.

$$
\begin{gathered}
F_{a}=F_{L C} \frac{L_{L C}}{L_{F a}}+W \frac{L_{C G}}{L_{F a}} \\
F_{L C}=F_{a}=F_{L C} \frac{L_{L C}}{L_{F_{a}}}+W \frac{\left(L_{C G}+\Delta L_{C G, g}\right)}{L_{F_{a}}}
\end{gathered}
$$

These equations are good to illustrate some of the points made previously. First off, the slope of the calibration curve is the inverse of the mechanical advantage from the design of the model mount. Second, the size of the y-intercept in the equation is proportional to the ratio of the CG offset distance and the distance between the pivot and the resultant force on the model, $\mathrm{L}_{\mathrm{Fa}}$. Since the $\mathrm{CG}$ offset is small compared to $\mathrm{L}_{\mathrm{Fa}}$, then for small changes in the CG offset, the y-intercept does not change very much. Even though it does not change very much, it is still accounted for in the data reduction. 


\subsubsection{Suction Slot Configurations}

The suction slot is tested with two different slot configurations. The first is a simple smooth slot. This slot has straight vertical sides at the slot entrance on the forward and aft faces of the slot. This type of slot is the same type as was used in the 1956 Goldschmied propulsor test. The performance of this slot is known to be below a cusp slot, but it is included in the current testing in order to compare with the data from that test as well as set a baseline for future improvements.

The cusp geometry included in the current round of testing is defined by Thomason ${ }^{20}$ and is shown in Fig. 3-20. Only one of the three cusps could be tested here due to tunnel time constraints. The cusp that is tested here is referred to as "Cusp A." The angle of the cusp tip with the freestream direction is $55^{\circ}$ and the tip extends 0.05 " into the flow.

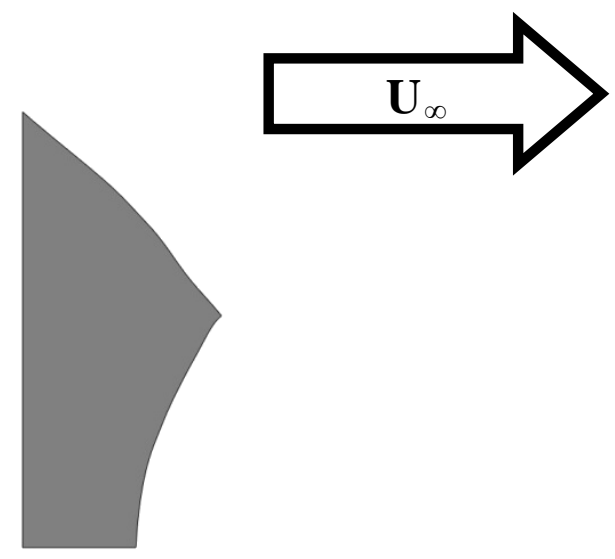

Figure 3-20. Cross section of the cusp used for the second half of the test matrix. The height of this section is approximately 0.4 " tall.

\subsubsection{SUCTION SLOT INTERNAL GEOMETRY}

The suction slot draws air in radially around the model. This radial flow of the air is important to reduce the influence of suction on axial momentum of the flow. The suction flow path separates the forbody from the aftbody of the model. The forward face of the suction flow path is the forbody, while the aft face of the suction plow path is the aftbody. The aftbody face is a vertical wall. Its shape is dictated by the placement of the fan, which needs to be as far aft as possible. This transition between the radial inflow and the axial flow through the fan needs to be as smooth as possible. Essentially, the flow needed to 'turn the corner' into the fan as gently as possible to prevent any flow separation. Flow separation upstream of the fan would have caused the fan to enter a stall-and-recover cycle. 
This stalled region would have caused the flow rate through the fan to decrease, which would have reduced the velocity of the air in the suction slot, this reduced velocity would allow the flow to reattach, and thus eliminating the blockage of the fan, which would have started the cycle over again. To allow the greatest chance that the flow would stay steadily attached to the short side radius entering the fan, a vertical wall with as large of a radius between the wall and the fan as possible was designed. This design can be seen in Fig. 3-21.

Another consideration was the fact that as the flow was moving radially, the cross sectional area of the flow is cylindrical in shape and decreasing in radius. The actual cross sectional area is proportional to both the radius and the width of the path at that radius. If both the forward and aft walls of the flow path were vertical, then the width would remain constant and the area would be solely proportional to the radius. This means that when the flow had reached half of the radius at the suction slot entrance, then the cross sectional area of the flow path would have also been reduced by half, and if analyzing along a streamline and outside of the boundary layer, the dynamic pressure of the flow would also be twice as much. This reduction in area of the flow path and increase in the dynamic pressure is thought to be detrimental to the performance of the fan. Therefore, to increase the area as the flow moved inward toward the fan, the width of the slot needed to be increases as the flow moved radially inward. The only side of the slot that was not fixed previously was the forward side of the flow path. Therefore, the forward side of the flow path tapers forward at an angle of $20^{\circ}$ from the vertical to keep the cross sectional area constant for a slot gap of $0.36^{\prime \prime}$. For larger slot gaps, the area actually decreases slightly in the flow direction, moreover, for smaller slot gaps, the area increases slightly in the flow direction. To keep the area truly constant, the slope would need to change with the slot width. This complexity was deemed unnecessary, so the average slot width was chosen as the best point to have a constant area. 


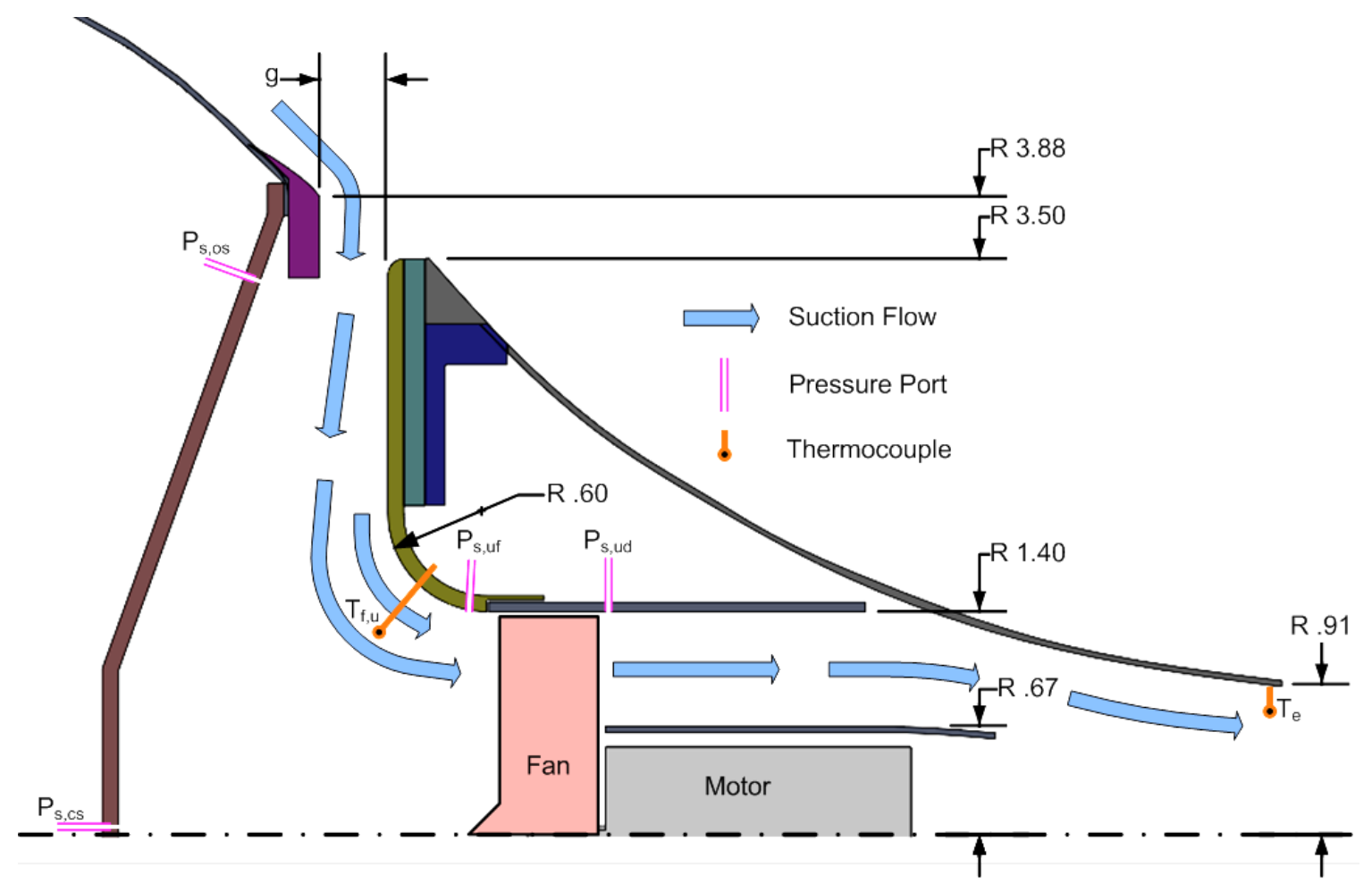

Figure 3-21. Diagram of the flow path of the suction flow.

\subsubsection{Suction Mass Flow Rate Measurement}

The suction mass flow rate is a difficult parameter to measure in this model. The method of measurement could not add any pressure loss to the suction flow, and it had to fit inside the model. Many methods were initially analyzed. Some of the ideas include a built-in turbine flowmeter, a built-in vortex shedding flowmeter, a built-in paddlewheel flowmeter, and various pressure based apparatus. The method with the best chance of success was a pressure-based method that measured flow rate using an empirical correlation between a pressure difference in the suction slot and a mass flow rate. Success in this context is a robust and accurate method of measuring mass flow rate. This type of pressure-based correlation is similar in nature to an orifice plate or venturi flow meter. The correlations exist for these devices, and the correlation coefficient is usually known as a "discharge coefficient." In these devices, the mass flow rate is proportional to the pressure difference between two locations, one upstream and one downstream, with different cross sectional areas. The pressure difference is measured and used with the correlation coefficient to calculate the mass flow rate. In the NGP, the upstream pressure port location is just inside the suction slot, close to the outer edge of the body, and it is called $\mathrm{P}_{\mathrm{S}, \mathrm{OS}}$, which stands for static pressure at the outer slot. The downstream pressure port location is on the centerline of the body directly forward of the fan, and it is 
called $\mathrm{P}_{\mathrm{S}, \mathrm{CS}}$, which stands for static pressure on the centerline of the slot. The actual location of these pressure ports can be seen above in Fig. 3-21. If the flow were completely axisymmetric inside the slot, then no flow would cross the centerline of the slot and there would not be any flow across the $\mathrm{P}_{\mathrm{S}, \mathrm{CS}}$ pressure port. Therefore, the pressure would be higher than at the slot entrance where the velocity over the port would be high in comparison.

To measure the mass flow rate to determine the correlation, a vane anemometer was attached at the end of a 36" straight rigid tube attached to the aftbody of the model. The tube was to allow the flow to increase in uniformity prior to passing through the vane anemometer. The correlation between mass flow and the slot differential pressure was determined as planned and then checked without an anemometer. When completing the check of the correlation, the power into the fan was held at the same level as one of the points in the correlation. When the pressure measurements were examined, it was noticed that the pressure differential did not fall on the correlation previously determined and that even the sign of the pressure differential was the opposite of when the correlation was determined. These observations lead to further investigations. These investigations lead to the determination that the vane anemometer produced a large amount of backpressure. This backpressure propagated upstream and influenced the pressures measured at the ports in the slot. Because of this effect, this method of measuring the mass flow rate while determining the correlation was eliminated. A less intrusive method of measuring mass flow rate was then utilized. The new method was to do a 9-point sweep with a pitot-static probe in the tube from one wall to the other wall. This would determine the velocity profile across the tube. This velocity profile was then integrated numerically to arrive at the mass flow rate. The equation used for this approach can be seen in Eq. 3.3. This method of measuring mass flow was successful at not influencing the upstream pressure measurements, and it was used for the remainder of the mass flow measurements during the pretest calibration process. It however was very time consuming to set up and complete.

$$
\dot{m}_{s}=\pi \int_{-R_{o}}^{R_{o}} \rho_{e} u(r) r d r
$$

Using this method, the correlation was determined for a single slot gap. To evaluate the robustness of the method, the correlation was then checked at multiple slot gaps. Unfortunately, the correlation that was determined at one slot gap was inaccurate for calculating the mass flow rate at other slot gaps. This demonstrated the need for a different correlation for each slot gap in the test matrix. So this was completed and the resulting correlation curves can be seen in Fig. 3-22. As can be seen from the figure, the method worked well at small slot gaps. The pressure 
difference between the upstream and downstream pressure port locations was distinct and varied appreciably with mass flow rate. It offered good resolution and was repeatable. It was also a relatively linear correlation with a very small y-intercept, which matches up with expectations. Unfortunately, these characteristics were not shared with the largest slot gaps. The most significant problem is that the pressure difference did not change appreciably with a significant change in mass flow rate. This resulted in little or no resolution in the signal. This was deemed unacceptable and therefore the pressure differential method of measuring mass flow rate was completely abandoned. However, the ports remained connected for all data points in the test matrix to allow for future evaluation and analysis.

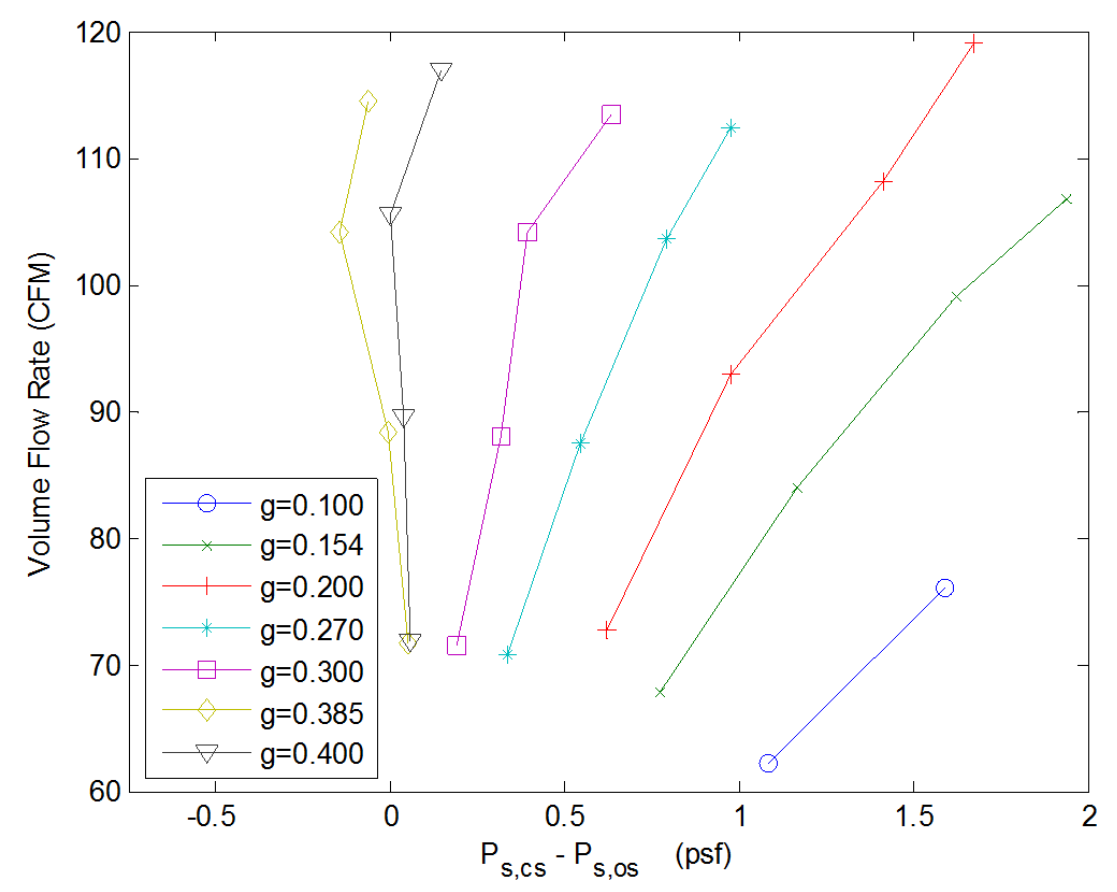

Figure 3-22. Initial suction mass flow rate calibration curves.

In its place, a survey with a pitot-static probe at the exit of the aftbody was used instead at each point in the test matrix. The survey consisted of measuring the velocity profile with the pitot-static probe at 6 points starting from the center of the aftbody exit and ending at a one-step distance away from the wall of the aftbody. This resulted in six measurements that were spaced nominally 0.15 " apart. A seventh point was added to the velocity profile that had zero velocity at the wall radius. The velocity profile was then assumed axisymmetric and numerically integrated according to Eq. 3.4. 


$$
\dot{m}_{s}=2 \pi \int_{0}^{R_{o}} \rho_{e} u(r) r d r
$$

The downside to this approach was the time it took to produce one mass flow rate measurement. The required testing time increased by a factor of six. Due to this, this method was abandoned and was not used in the final data set presented later. The mass flow rate was calculated by using a single velocity measurement at the exit of the aftbody. The probe was placed 0.65 " from the center of the exit area. This was chosen to represent the average velocity of the exit based on what was learned when the 6-point surveys were being competed. This average velocity was then used to calculate the mass flow rate according to Eq. 3.5.

$$
\dot{m}_{s}=\rho_{e} A_{e} \bar{V}=\left(\frac{P_{s, e}+P_{0}}{R T_{e}}\right) A_{e} \sqrt{\frac{2 R T_{e}\left(P_{t, e}-P_{s, e}\right)}{P_{s, e}+P_{0, a b}}}
$$

\subsubsection{Fan Pressure Rise Measurements}

One important parameter that was desired to analyze the fan performance in the model was the pressure rise across the fan. The fan pressure rise as measured is more specifically a static pressure difference. The upstream static pressure was measured approximately 0.1 " upstream of the leading edge of the fan blades on the wall surface on the aluminum piece upstream of the fan. A pressure port was mounted every $90^{\circ}$ and all four of these ports were connected together with a manifold to provide an average value to the single channel on the Scanivalve. The downstream port was measured in the same averaging manner, except it was mounted about 0.1 " downstream of the fan trailing edge. These ports and the general layout of the aftbody can be seen above in Fig. 3-21 and Fig. 3-23. 


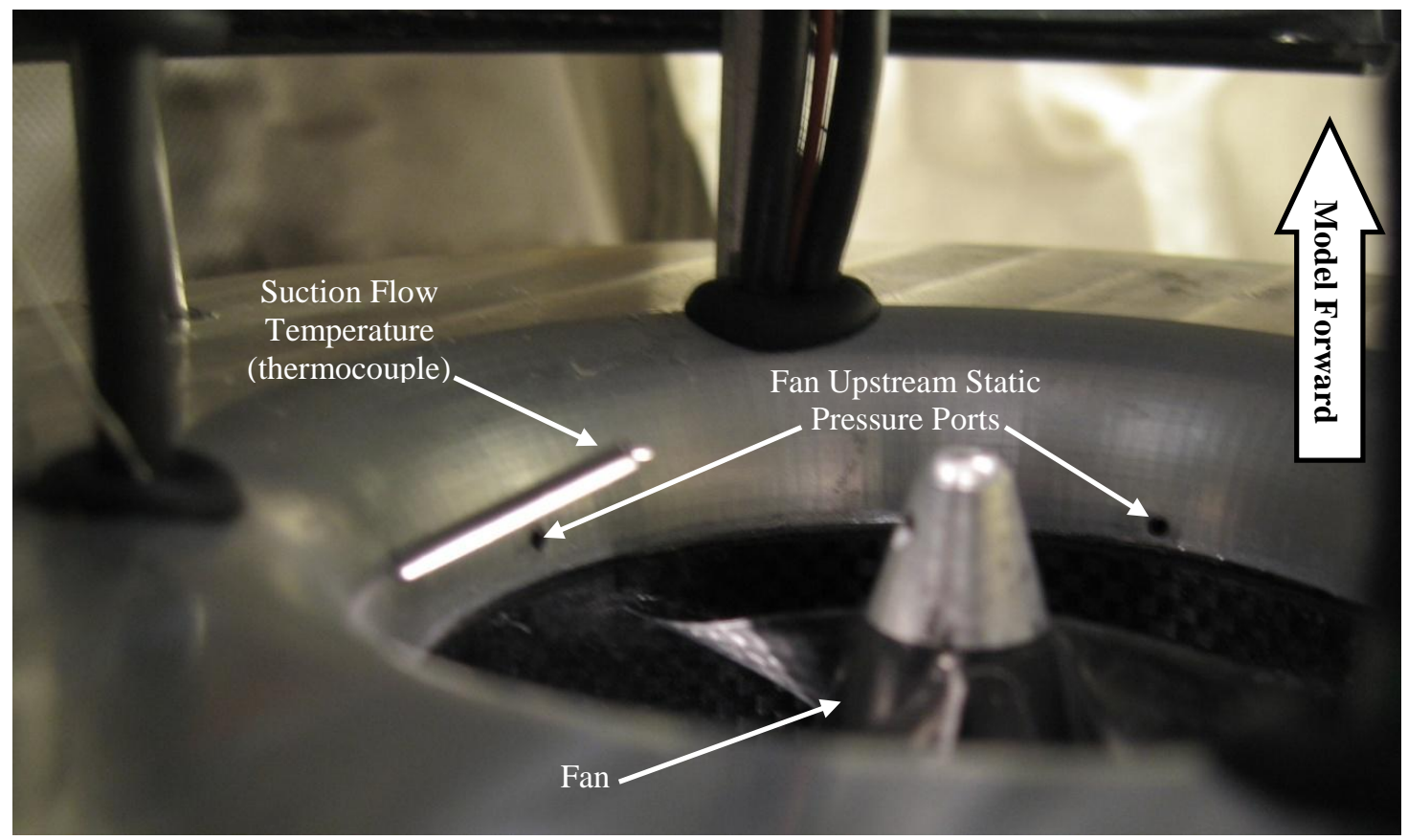

Figure 3-23. Picture of the fan upstream pressure ports and suction flow thermocouple.

\subsubsection{FAn TEMPERATURE RISE MEASUREMENTS}

Another desirable parameter from the standpoint of fan performance was the temperature rise across the fan. It is important because the electric motor is cooled by the suction flow, and any rise of the flow temperature would result in a change in the density in the suction mass flow and possibly the exit speed. This effect was difficult to estimate, so the ability to measure the flow temperature upstream and downstream of the fan was added. The upstream temperature was measured by a single 0.033 " diameter type K thermocouple that protrudes about 0.5 " into the flow through a stainless steel tube bonded in place for this purpose. The distance between the thermocouple tip and fan leading edges is about 0.6 ". The downstream temperature was measured with the same type of thermocouple, but it was taped to the inner wall of the aftbody skin at the exit to the aftbody and was bent to protrude about 0.2 " into the flow. It was mounted as far down stream as possible to try to capture the maximum temperature rise possible. The locations of the thermocouples can be seen in Fig. 3-21 and Fig. 3-24. In general, the temperature rise never exceeded $7^{\circ} \mathrm{F}\left(3.9^{\circ} \mathrm{C}\right)$. 


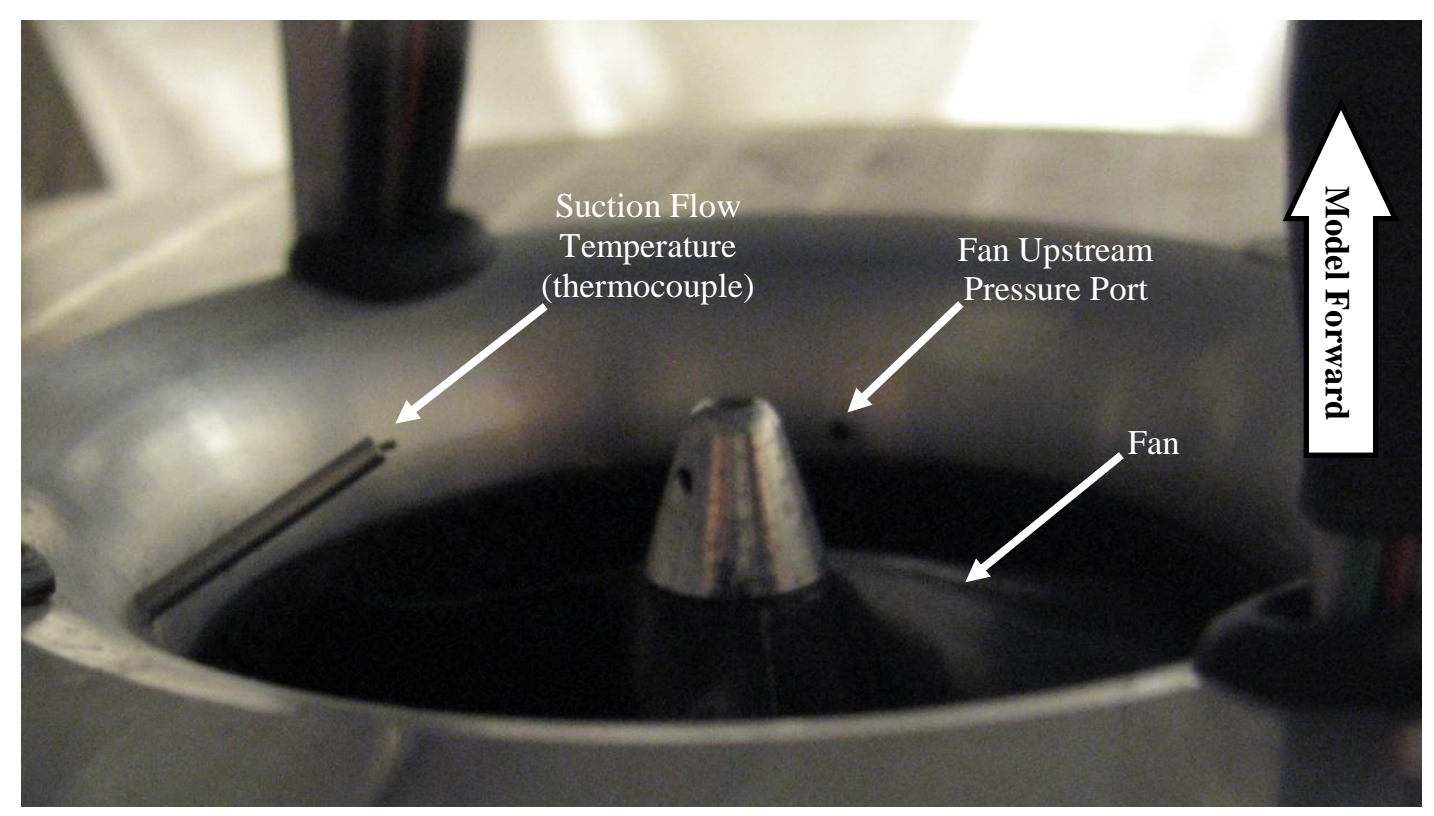

Figure 3-24. Picture of the suction flow thermocouple just upstream of the fan.

\subsubsection{FAn ThruSt \& FAn TORQUe MEASUREMENTS}

Fan thrust and torque were each measured through two Omega Thin Beam Load Cells. These load cells have a maximum load of $1 \mathrm{lb}(4.445 \mathrm{~N})$ each. This allows a maximum thrust of $2 \mathrm{lbs}(8.890 \mathrm{~N})$ and a maximum torque to be $3.14 \mathrm{in}-\mathrm{lb}(0.355 \mathrm{~N}-\mathrm{m})$. The load cells themselves are full Wheatstone bridge type sensors. They have a sensitivity of $2 \mathrm{mV} / \mathrm{V}$ and are temperature compensated between $20^{\circ} \mathrm{F}$ and $120^{\circ} \mathrm{F}$. They have a stated combined error band, including hysteresis, linearity, and repeatability, of $0.25 \%$ of full scale, or $0.0025 \mathrm{lbs}(0.011 \mathrm{~N})$. The design configuration of the load cells in the model can be seen in Fig. 3-25. The actual load cells mounted in the model can be seen in Fig. 3-26 and Fig. 3-27.

The load cells do not work as a traditional S-beam, or even a traditional bending beam load cell. A traditional load cell has a rigid beam that has an area that is thinner where the strain gauges are mounted because the strain is concentrated there. The load cells used in the NGP model operate on the simple principle of a flexible beam in bending. The flexible steel beam bends when a force is applied to one of the beam ends while the other is help fixed. The force is applied normal to the plane of the beam and causes it to have two bends with an inflection point between them. All strain gauges are mounted on one side of the beam, and the two strain gauges that are in tension are on the outside of a curve, and the other two strain gauges are on the inside of the other curve causing them to be in compression. 

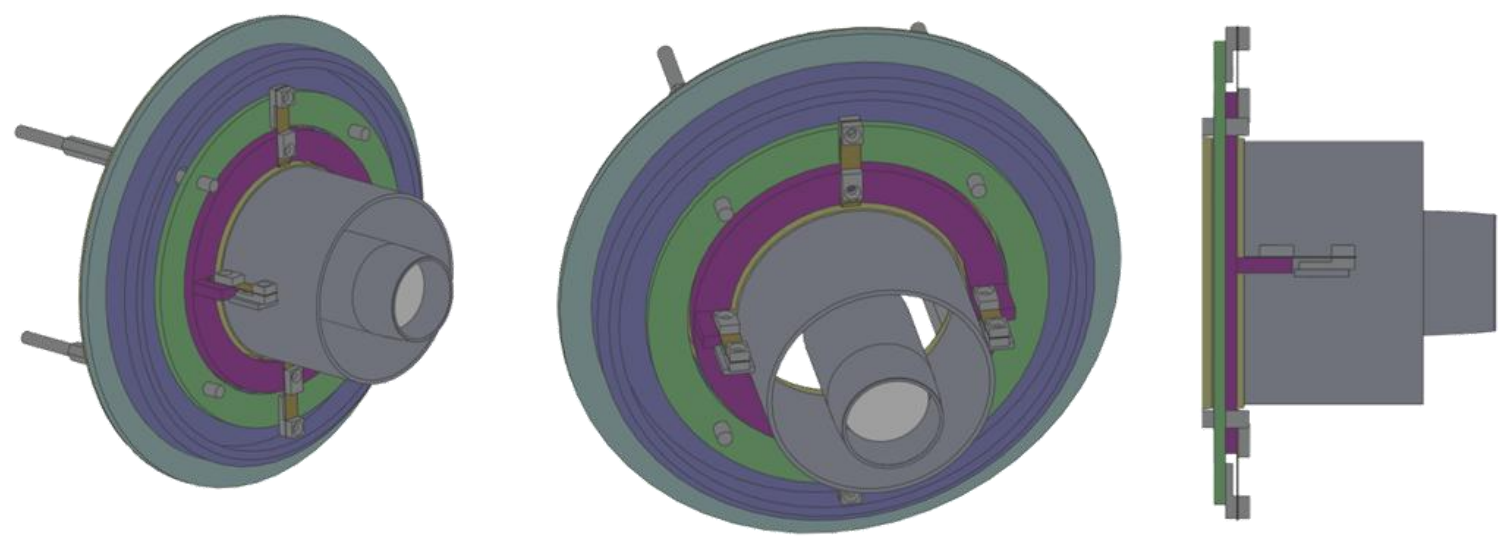

Figure 3-25. CAD model of the load cells mounted in the aftbody of the model. The horizontally mounted load cells measure fan torque, and the vertically mounted load cells measure fan thrust.

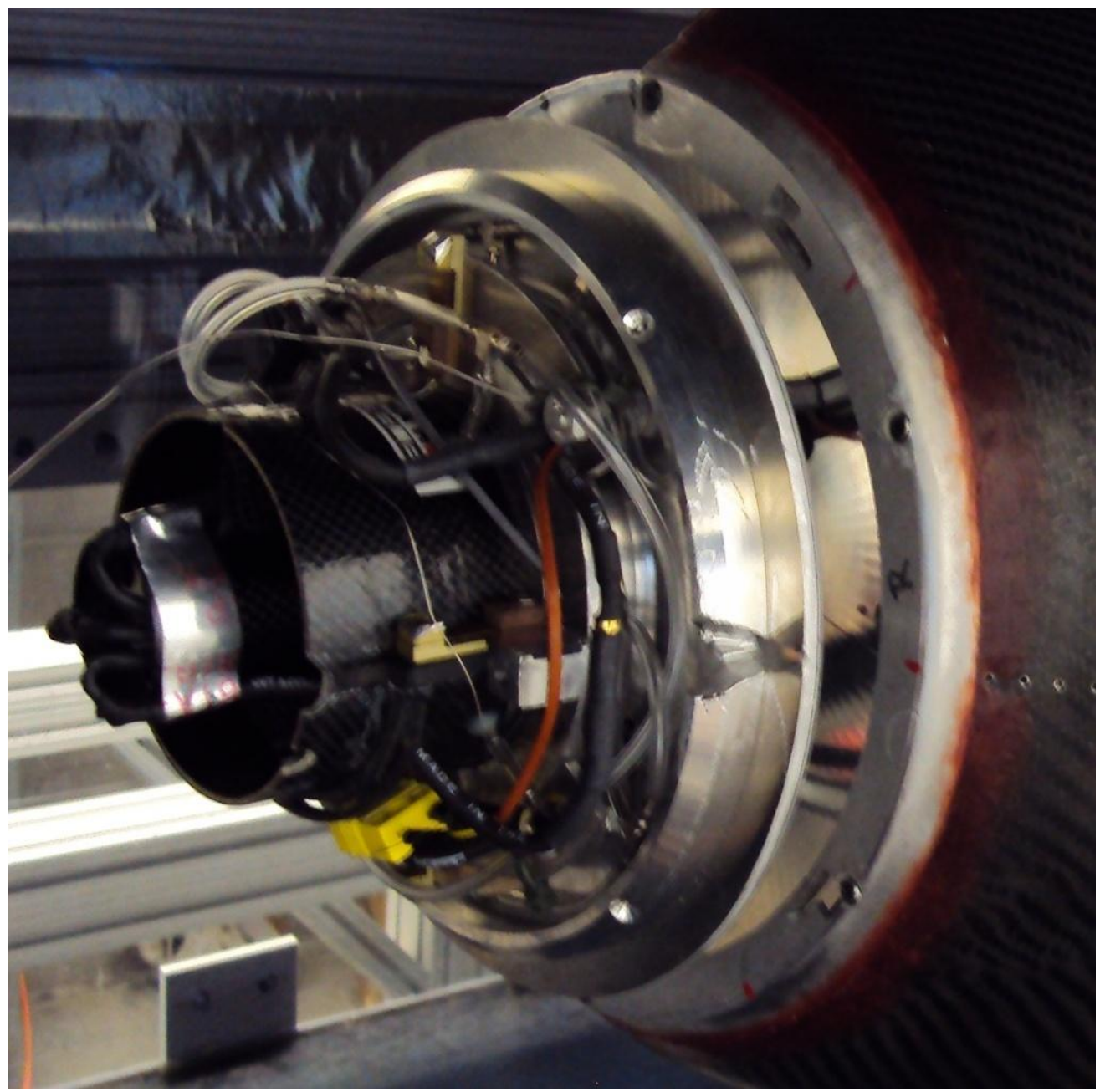

Figure 3-26. Actual installation of the load cells in the aftbody with the wiring and tubing routed around them. 


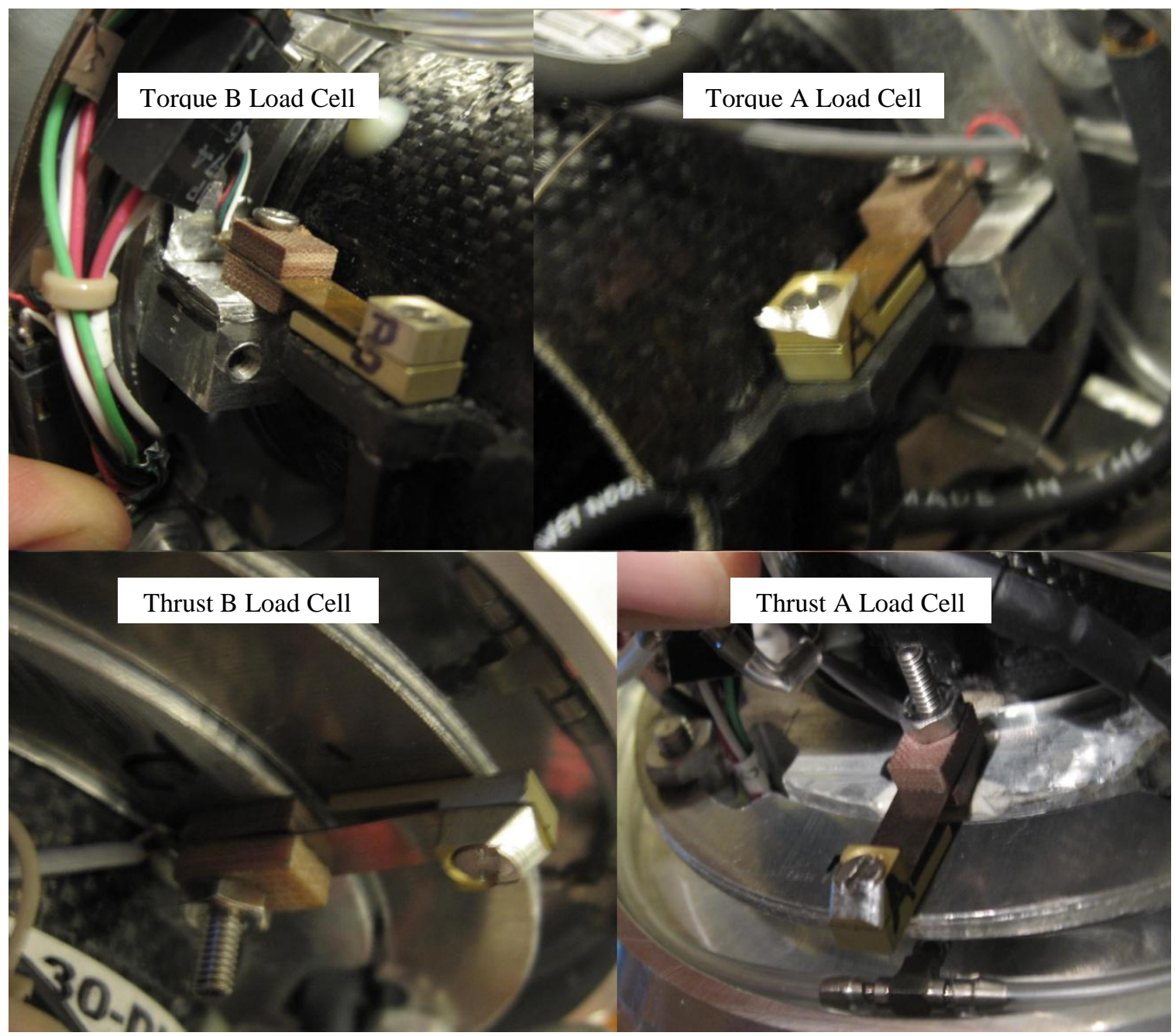

Figure 3-27. Close up views of the load cells installed.

\subsubsection{Fan Power Measurements}

Power is simply work (energy) done in a given amount of time. The power delivered to the fan comes from a DC power supply. The power that the power supply delivers is calculated by measuring both the current and voltage at the supply's output terminals. As discussed previously, this power supply is an HP 6012b made by Hewlett Packard. Direct measurement of the voltage by the data acquisition system is not possible because the maximum input voltage is $10 \mathrm{~V}$ and the supply output voltage is $16.8 \mathrm{~V}$. To accomplish higher voltage reading with the data acquisition system, special high voltage input modules that scale down the voltage are available. To measure current, there are two common options. The first option, a shunt resistor, is a simple resistor with a very precisely known resistance. Then the voltage drop across the resistor is measured and the current through the resistor 
is calculated from Ohm's Law. The losses due to the additional resistance in the system are very undesirable because of the reduction the actual fan power. The second option is a non-intrusive inductive current probe. This option is accurate, but expensive to find one that can interface with the data acquisition system. A low cost alternative that just has a digital read out of the current was used for calibration purposes, but not for the current measurement during testing. Ultimately, none of these options are needed because the fan power supply has the built in capability to output two $0-5 \mathrm{Vdc}$ signals that corresponds to $0-50 \mathrm{~V}$ and $0-60 \mathrm{~A}$, respectively. The calibration of these channels is discussed in Section 4.1.5.

The power that is measured at the power supply will be transferred and transformed many times by the time it has dissipated into heating the air outside of the tunnel. It is important to differentiate and clearly define all of the different forms the power will take along the way. There is electric power consumption at the motor, shaft power, and fan power to name the most important. The fan DC motor consumes power that exits the power supply, but not all of that power is consumed by the motor. Some is consumed in heating the wiring and other components between the motor and the power supply. Some of that power is consumed by the ESC to accomplish the data logging, speed control, and timing tasks. These losses are estimated at $5 \%$ of the total power consumption. Therefore, the fan motor consumes $95 \%$ of the power that exits the power supply.

The motor consumes this electrical power, but not all of that power is delivered to the shaft of the motor to do mechanical work by the fan. It is assumed that the motor is $90 \%$ efficient. This means that $10 \%$ of the power that reaches the motor is lost to heat and friction.

These two efficiencies result in the fan receiving $85.5 \%$ of the power that has been delivered by the power supply. The fan takes this power and transfers it to the airflow in the form of a static pressure increase. Another way to think about it is the fan absorbs the power from the motor shaft and uses it to turn the fan blades. The fan blades are just rotating wings that are passing through air. These wings produce lift and drag. These two forces combine to form a resultant force. The component of this force that is tangential to the blade rotation is measured as torque, and the component of this force that is along the axis of rotation is the fan thrust. These forces are the result of the pressure forces and skin friction forces on the fan duct and rotating blades. These forces are transferred through the fan duct, and finally through the fan mounts and into the NGP. The fan exerts an equal and opposite force on the air 
as it moves through the fan duct. The torque causes the airflow to swirl in the duct as it travels downstream. The thrust force acting over the area that the airflow moves through is the pressure increase that the flow experiences.

\subsubsection{Surface Pressure Measurements}

The pressure force of the model is calculated from the static pressure ports on the outer surface of the model. In total there are 56 pressure ports used for this purpose, some of which are shown in Fig. 3-28. The pressures at these ports were recorded by the Scanivalve system in the wind tunnel, which is discussed further in Section 3.3.1. The ports are arranged in a straight line on the horizontal center plane of the right half of the model. They were placed on the right half of the model to minimize any effects from the traverse and probe mount, which are on the left side of the model. In addition, the left and right halves of the model are farther away from the wind tunnel walls then the top of the model; therefore, they would be less affected by the walls than the top.

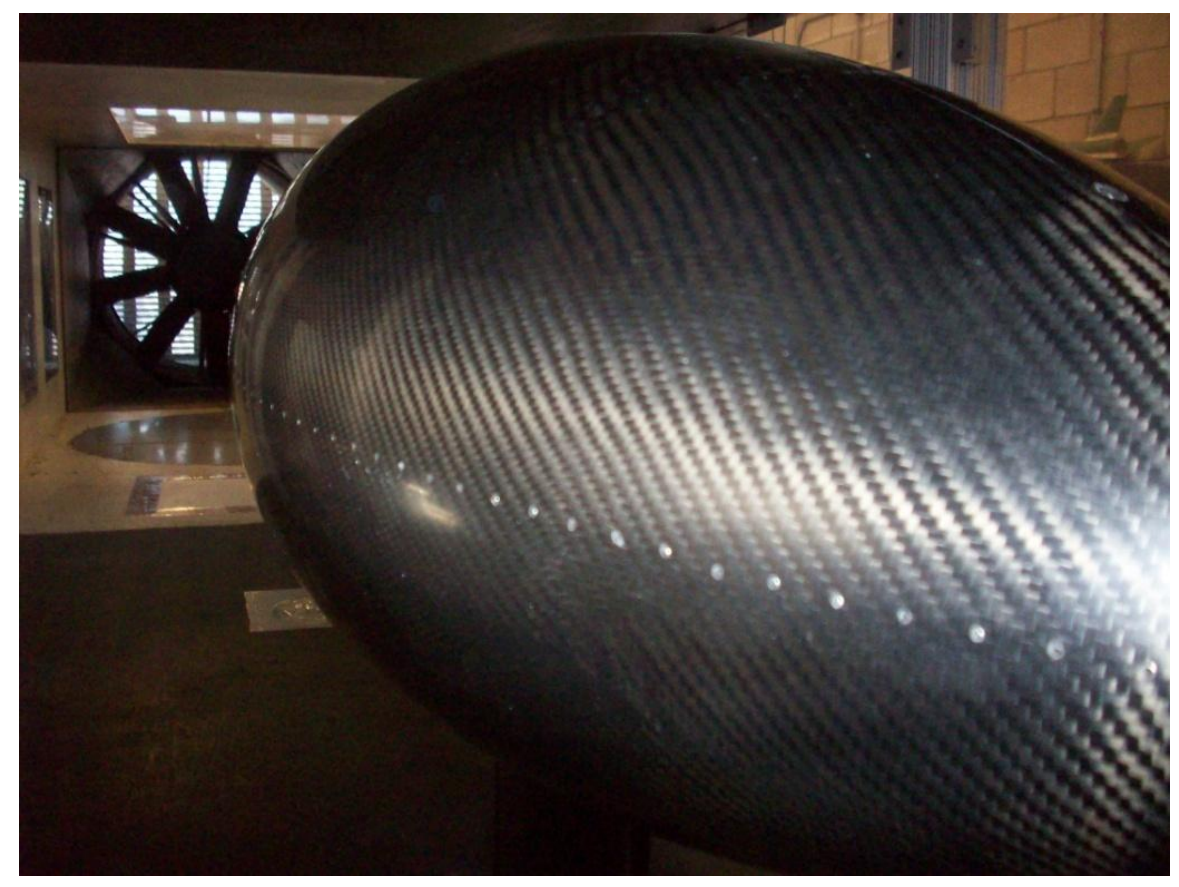

Figure 3-28. Surface of the NGP showing the surface pressure ports used to calculate the axial pressure force on the model.

The derivation of the pressure force equation assumes each pressure port location is the intersection of two panels on the model surface. Each panel is assumed to take the shape of a frustum of a cone. A frustum of a cone is what is left when the tip of the cone has been cut off by a plane parallel to its base. The surface area of the frustum of the cone is calculated from the known locations of the pressure ports at its edges. The pressure on the frustum 
surface is the average pressure read by the pressure ports at its edges, and it is assumed constant over the entire outer surface area of the cone frustum. This pressure over an area produces a resultant force that is perpendicular to the surface. The component of that force in the axial direction is the axial pressure force on the body for that frustum. The axial force component is determined from the axial and radial spacing of the pressure ports on its edges. The total axial pressure force on the body is the summation of the forces from the individual frustums. The calculation of the axial pressure force coefficient is given in Eq. 3.6.

By examining this equation, it is evident that the spacing of the ports radially on the body influences the calculated pressure force on the body. It shows that not only is the actual radius of the ports important, but the difference between the ports radially is equally important. Therefore, a panel can have a high influence either if it is close to the maximum thickness of the body or if the ports' radial spacing is large. Therefore, it is important to look at both of these aspects of the port spacing when determining the port spacing on the model. More specifically, the bracketed term in Eq. 3.6 is completely determined by geometry and determines the relative influence of a given panel on the overall axial pressure force on the body. The pressure port locations are given graphically in Fig. 3-29 and numerically in Appendix D. The surface of the model was divided into three regions: the forward part of the forbody, the rear part of the forbody, and the aftbody. Each region is delegated a certain number of ports and an axial distance that the ports must span. The growth ratio between the pressure ports' axial locations is iterated until the ports are distributed fully across the allotted space with a constant growth ratio that leaves a clustering near the leading edge and the slot. The numbers of ports on each part of the body and the exact location of the split on the forbody were manually iterated until the relative influence between panels was not concentrated in any one region.

$$
C_{a, p}=\sum_{i=1}^{N-1}-\left(\frac{C_{p, i+1}+C_{p, i}}{2}\right)\left[\frac{\pi\left(r_{i+1}^{2}-r_{i}^{2}\right)}{A_{r e f}}\right]
$$

By examining Fig. 3-29, one can see that the forward ports on the forbody have a negative influence on the axial pressure force and the rear part of the forbody and the aftbody have positive influence on the axial pressure force. This matches with expectations, and if a positive pressure were assumed everywhere on the model for simplicity, then the sign of the force on each section of the model would match the sign of the influence of the panel. This matches with the sign convention for axial force, as it is positive in the forward direction of the model. 
The equation was derived from the principles of the pressure drag, but by inspection of the final equation, a much simpler derivation would have sufficed. The equation indicates that the area that the pressure acts on is a ring with an outer radius and an inner radius given by the radii of the outer and inner ports, respectively. The normal vector of the ring points in the axial direction. Effectively, the summation is over the front and back of a disc with the same radius as the maximum radius of the model. Due to this significance of this frontal area of the model, the reference area used in the calculation of the axial pressure force coefficient, and all other coefficients that use a reference area, is the frontal area of the model.

Originally, a wake survey was going to be completed at a few select data point in the test matrix. If two channels on the Scanivalve were designated to be used for this wake pitot static probe, they would not have been used for more than $95 \%$ of the test matrix. Therefore, instead of letting two ports go unused for most of the testing, it was decided that for the few data points where a wake survey was going to be completed, that these data points would be taken twice. The first time with all the Scanivalve channels connected to their normal locations and the second time with the two channels that were originally reading static pressure before and after the fan to be repurposed for the pitot static probe. This strategy changed when the need arose for two additional channels on the Scanivalve for every point in the test matrix, instead of just a few. These ports were needed for the pitot static probe to measure mass flow rate for every data point in the test matrix. Instead of losing the ability to measure the static pressure before and after the fan, the decision was made to not measure surface pressure on two of the ports on the model surface and repurpose these channels instead for the pitot static probe to measure suction mass flow rate. These channels were ports \#29 and \#31 on the model surface. They are circled in the plots of Fig. 3-29. These ports were chosen because, as can be seen in the lower figure, they are connected to frustums that have the lowest influence on the axial pressure force. Now that these two ports were being used for another purpose, there are 54 ports used for the pressure force calculation. 

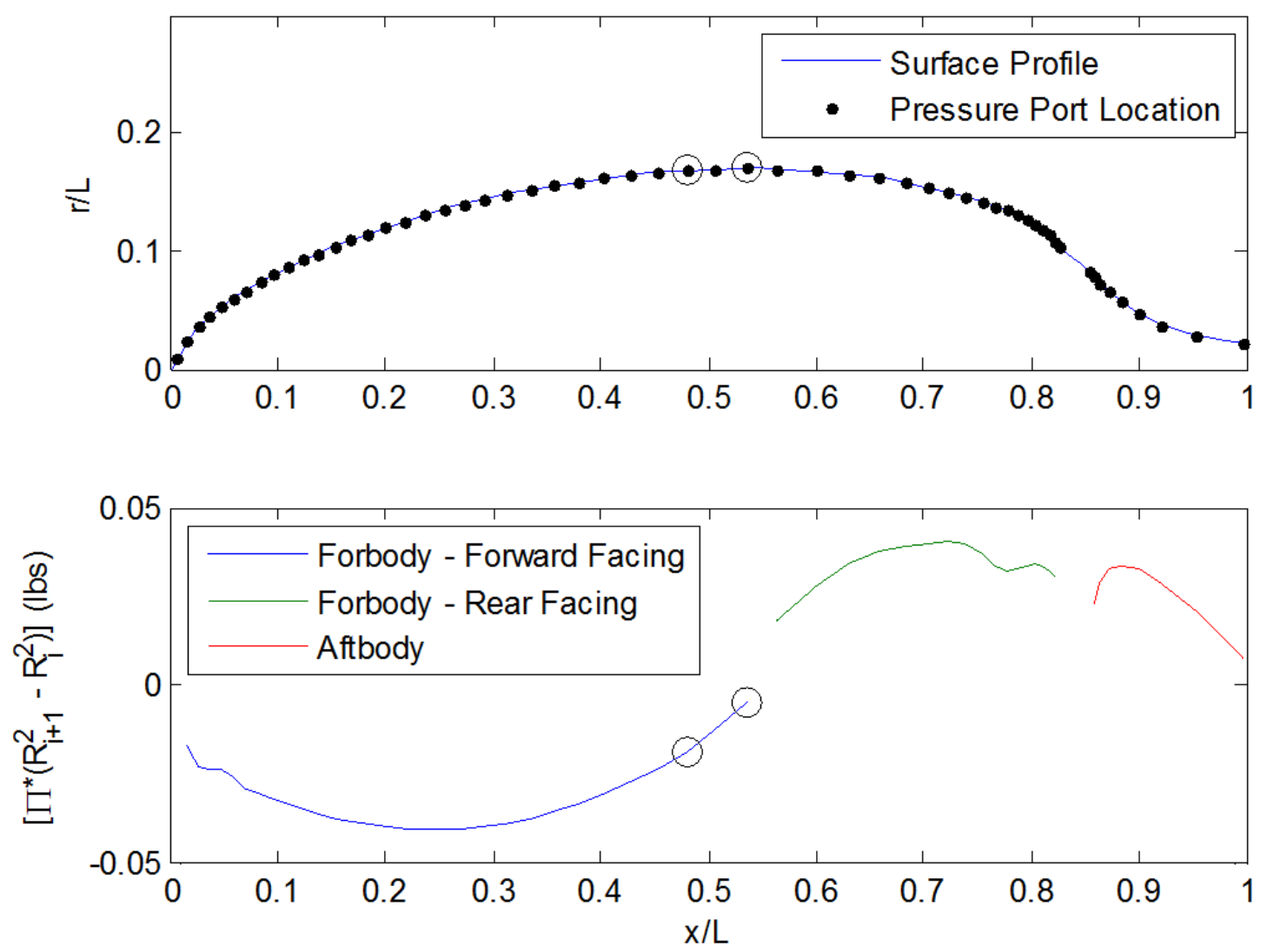

Figure 3-29. Designation of the surface pressure port locations (Top) and their relative influence on the overall pressure force (Bottom). The circled ports ended up being used for other measurements during testing.

\subsubsection{Model Health Monitoring}

If possible, the test/model designer wants a way to determine if something has gone wrong with the model during the testing. One of the possible problems is a mount coming loose after a long period of running the tunnel. This could be caused by the vibration of the model or even changing atmospheric temperatures. To record if, and when, this had occurred during a block of testing, two 2-axis accelerometers are mounted in the model. One is place on the upper model strut mount with its axes pointing axially and vertically, referred to as X1 and Y1. The other was mounted on the traverse mount at the rear of the forbody. The axes of this sensor were pointed axially and horizontally, and were referred to as $\mathrm{X} 2$ and Z1. The alignment of the sensors is not critical, as they are more for comparison purposes than to measure the exact acceleration. The accelerometers used have a bandwidth of $500 \mathrm{~Hz}$ and a stated accuracy of less than $1 \%$ of the 1.7 Gs full scale. 
As a second health monitor, a thermocouple was taped to the outer surface of the fan motor to monitor the temperature of the fan motor during extended blocks of testing. From conversations with experienced RC pilots and enthusiasts, a safe operating temperature limit is set at $150^{\circ} \mathrm{F}\left(65.6^{\circ} \mathrm{C}\right)$. This precaution is deemed necessary because of the normally short durations of use for these motors in an RC aircraft application compared to this application. It was advised by the manufacturer to add cooling vents to the motor housing to aid in cooling if required, but due to their unknown performance impact to the fan and suction flow rate, they were not utilized.

\subsubsection{Slot GapAdjustment Control}

The suction slot width, or slot gap, dictates the amount of area available for the suction flow to enter the fan. The larger the slot gap, the more area open for the suction flow and, theoretically, the lower the velocity of the suction flow at the slot entrance. In addition, the larger the slot gap, the more gradual radius that the suction flow could travel and still enter the suction slot. To adjust the slot, the entire aftbody is moved relative to the forbody. To carry the weight of the aftbody, fan, motor, and associated hardware, a small traverse was used. A traverse is a mechanism that allows precise movement of whatever is mounted on the traverse mounting block. The traverse consists of a block that slides on a set of precise rails, and it is propelled by the use a threaded rod. The threaded rod is rotated by a stepper motor, in this case, to allow for very precise control of the motion and high torque at low rotational speeds. The increment of movement is dictated by the smallest step size of the motor and the thread pitch of the threaded rod. For the traverse used in the MGP, the stepper motor moves in 1.8 degrees per step, and the threaded rod has a pitch of 40 threads per inch. These two together create system that can move in increments of 0.000125 " per step. Also by using a stepper motor, the motor has enough torque to overcome the friction in the rail mechanism and any additional friction due to the aftbody sliding past the stationary forbody.

A linear 5.1 kilo-ohm potentiometer was mounted to the traverse and provided feedback as to the actual traverse position throughout the range of movement of the traverse. This potentiometer reading was recorded in the National Instruments data acquisition system. The slot gap was calibrated prior to any testing by moving the slot to various widths and measuring the opening with digital calipers at multiple locations around the body, averaging these measurements, and plotting these values against the recorded output voltage from the potentiometer. It is worth noting that the slot gap varied from top to bottom by less than 0.017 " $(0.43 \mathrm{~mm})$, and the variation was consistent for all slot gaps. It did not vary appreciable side to side, or in any other direction. 


\subsubsection{FAN SPEEd CONTROL}

The EDF relies on an electronic speed controller (ESC) to control its speed during operation. In its designed usage, the ESC takes in a 'throttle' signal from the model airplane receiver and modulates the DC battery voltage to an AC voltage. The RMS of this AC signal is proportional to the throttle input. The modulation is a pulse width modulation, which for anything besides $0 \%$ throttle and $100 \%$ throttle, is a type of square wave AC voltage signal.

The ESC chosen for this project is by a company Castle Creations. It was chosen because it has the ability to read and record the EDF's rotational speed. It does this through the back electromotive force (EMF) of the motor. The back EMF is an indication of the actual motor rotational speed, in opposed to the commanded speed. It is caused by the changing magnetic field of the motor inducing a voltage in the motor windings when there is no voltage applied to them by the ESC. The ESC then records this value at a frequency of $10 \mathrm{~Hz}$. The ESC has limited memory for data storage. With a recording frequency of $10 \mathrm{~Hz}$, the ESC can hold about 20 minutes of recorded data. In addition, the ESC is recording the entire time that it has power. This became the limiting factor in the length of a block of time spent recording data.

The ESC in this test takes its throttle signal from a servo tester instead of receiver. This takes advantage of the fact that signals for a traditional analog servo and the ESC are identical in shape. The servo tester is a device that connects to the user's computer through USB and allows the manual setting of the servo position from 0-100\%, but when connected to an ESC, this corresponds to 0-100\% throttle.

\subsubsection{Transition TripStrips}

The NGP trips the boundary layer at $10 \%$ of the body length. The reasons for this are given in Section 2.7 . The local velocity outside the boundary layer was estimated to be between $68 \mathrm{ft} / \mathrm{s}$ and $72 \mathrm{ft} / \mathrm{s}$. A roughness Reynolds number of 600 results in the minimum roughness height being 0.0013 " and 0.0014 ". This is much less then the thinnest boundary layer trip strip material found that is 0.020 ". This was deemed acceptable because no thinner material could be found. To add to the 3D nature of the trip strip, it was cut with a jagged, or saw-toothed, leading and trailing edge. This is called pinking, or a pinked edge. The average width of the trip strip is $1 / 4$ ".

The effectiveness of the boundary layer trip strip was evaluated using an auditory technique. A total pressure probe on a long handle was connected to a stethoscope through a length of flexible tubing. The probe was slowly 
moved from the forward tip to the aft end of the model while the tunnel was running and the author listening to the stethoscope. The sound heard in the stethoscope is simply amplified pressure oscillations picked up by the total pressure probe. When the probe was placed in the laminar boundary layer forward of the trip strip, the noise heard in the stethoscope was very quiet. The low amplitude noise was heard for all locations forward of the trip strip. Aft of the trip strip the turbulent boundary layer had a distinctly louder noise with a different pitch. This louder noise was heard on the entire forbody aft of the trip strip and even on the aftbody. For this test, the model fan was not running. Based on these results, the boundary layer was considered to be turbulent everywhere aft of the trip strip, and the trip strip was deemed effective.

\subsection{DATA ACQUISITION SYSTEMS}

The data acquisition system for this test was completely electronic. It consisted of four separate parts. These were the National Instruments (NI) system, Measurement Computing (MC) system, Scanivalve pressure measurement system, and the Castle Creations (CC) electronic speed control (ESC) system.

\subsubsection{Scanivalve SySTEM}

The Scanivalve system consists of a Scanivalve ZOC 33/64 Ethernet based pressure-scanning module. This device has eight modules with eight channels per module. Four of the modules have an input ranges of \pm 10 inches of water $(0.3613$ psid $)$, while the other four modules have a range of \pm 1 psid. The stated accuracy of these channels is $\pm 0.15 \%$ of full scale $( \pm 0.00054$ psid $)$ and $\pm 0.12 \%$ of full scale $( \pm 0.0012$ psid $)$, respectively. This system does not read all channels simultaneously. It reads one channel at a time with a period between reading consecutive ports. This period can be set as small as $50 \mu \mathrm{s}$. It reads all 64 ports every scan. These two characteristics combine to allow a maximum scan rate for each port of $312.5 \mathrm{~Hz}$. For all data points in this test, the Scanivalve was configured to record at this rate See Appendix E for a complete description of how to operate the Scanivalve.

The pressure ports were thoroughly checked for leaks prior to connecting them to the Scanivalve. The general procedure for this is to place a piece of sealant tape over the surface pressure port and apply a vacuum of at least 20 in- $\mathrm{Hg}$ with a hand-help vacuum pump. The gauge on the vacuum pump was observed for at least 5 seconds and if the vacuum dropped more than $1 \mathrm{in}-\mathrm{Hg}$ in that time frame, then the port and tubing were investigated and the leak fixed. This was important to achieve reliable results. 


\subsubsection{NATIONAL INSTRUMENTS SYSTEM}

The NI system was responsible for the bulk of the data recording. It consisted of a PCI-6259 High Speed Data Acquisition card that was mounted on the motherboard of the lab computer. This card is capable of 1 million samples per second across all 32 of its analog inputs. It has a16 bit analog to digital converter, and it has selectable gains on the input channels, which allow input ranges down to $\pm 0.1 \mathrm{~V}$. This allows the device to resolve voltage changes as small as $3 \mu \mathrm{V}$ theoretically and $6 \mu \mathrm{V}$ practically (as state in the component specifications). The 32 analog inputs are what are referred to as single ended inputs. They are referenced to ground. Two single ended inputs can also be configured as one double-ended input. This type of input measures the voltage between the two channels and is not referenced to ground. This allows a voltage measurement where neither side of the measurement is tied to the ground of the data acquisition system. This type of measurement input was used for the five load cells in the model.

One example of where this was important was in the measurement of axial force. The load cell uses a full Wheatstone bridge. The Wheatstone bridge was supplied with nominally $10 \mathrm{Vdc}$. The output of the sensor is the voltage read across the middle of the bridge. The ground of the $10 \mathrm{Vdc}$ is tied to the ground of the National Instruments system. If a single ended input were used to read this voltage, it would effectively short out one leg of the Wheatstone bridge. This would at least considerably change the output reading of the sensor, and, at worst, could damage the sensor.

Besides the PCI card in the tower of the computer, the system is composed of two $16.4 \mathrm{ft}$ cables that connect the board to two shielded terminal blocks. The terminal blocks are the point of connection for all input channels. They use screw terminals to attach the wires. It also utilizes a strain relief clamp for all the wires. This prevents any damage to the terminal block if any of the wires are pulled. It also allows for a more robust connection between the wires and the terminal blocks because the wire is less likely to shake loose due to vibration.

The terminal blocks also provide a shielded connection between the data acquisition system and the sensors. Shielding of signals is a very important and often overlooked part of data acquisition. The shielding inhibits any electromagnetic interference (EMI) from inducing electrical potential on the sensor wires. This EMI induced potential usually shows up as noise in the voltage readings. In the testing environment of the wind tunnel, there exists considerable EMI due to the large 3-phase electric motor and associated variable frequency drive (VFD) used to drive it. Besides the terminal blocks, the wires used for all sense and control signals utilized a metallic foil shield 
and uncoated ground wires inside the cable bundles. These two items are to shield the wires inside the cable from the EMI, but if any EMI does leak through, the wires inside the cable are twisted pairs of 22 AWG wire. By utilizing twisted pairs of wire, any EMI that is able to penetrate the shielding is canceled out by inducing effectively equal interference into both wires of a twisted pair. By inducing equal interference into both wires, the voltage between the wires stays constant. For all wiring, the uncoated shield wires were tied together between multiple wires to reduce the number of pins and connectors both in the model and below the wind tunnel. The shield wires were grounded outside the tunnel at the terminal blocks. Ideally, the shield wires would be grounded close to the sensors, but this was not possible because there was not a good ground inside the model.

\subsubsection{MEASurement CoMPuting System}

The Measurement Computing (MC) system connects to the computer with a USB connection. The unit used is model number USB2416. It is capable of 1000 samples per second across all 16 of its 24-bit analog inputs. Like the NI system, these inputs can be configured for 16 single ended or 8 double ended analog inputs. Because of its much higher resolution analog to digital converter (ADC), this unit could read and record unamplified thermocouples. It also had an internal thermocouple reference sensor to allow for accurate measurements with thermocouples. Therefore, this unit was used to record all thermocouple data. It was not used to record any other data because of the fact that the signal connections are not shielded and the recording rate is shared and would be slower if more channels were connected to it. Due to the lack of shielding, the thermocouple signals contained more EMI noise than the NI signals.

\subsubsection{SPEED CONTROLler SySTEM}

The speed controller, or ESC, recorded the fan rotation speed on memory internal to the device. It is made by a company called Castle Creations, and it is the model Phoenix Ice 75 . It is rated at a maximum current of 75 amps. The available memory is limited, so the unit can only record about 20 minutes of data at a maximum recording frequency of $10 \mathrm{~Hz}$. Once its memory is full, it overwrites the oldest data in memory. 


\section{ANALYSIS}

The analysis of the NGP encompasses both the calibration process and the actual data reduction process. The calibration process involved in-place calibrations of the axial force load cell, fan thrust load cells, fan torque load cells, the slot gap potentiometer, and the fan voltage and current.

\subsection{CALIBRATIONS}

The calibration of the required channels resulted in the need for purpose built equipment to complete the tasks. The design of which was done as the need for the equipment arose. This resulted in the use of local and available materials and equipment, which was the best option at the time due to tunnel time constraints. The equipment was evolved until the resulting calibrations of the individual parameters were qualitatively repeatable. Once the calibrations were repeatable, the equipment and calibration process were repeated for all subsequent calibrations.

To examine the accuracy of each calibration, the calibration points were repurposed as test points and were evaluated by the calibration functions. The error between the known quantity and the calculated quantity is called the residual. The absolute value of these residuals is shown for all calibration curves. The average of the absolute value of the residuals is used in some cases as the estimate of the systemic error in the measurement.

\subsubsection{AXIAl Force CAlibration}

The calibration of the axial force load cell was a process that went through several iterations. The initial process used traditional pulleys and weights to either apply a load in the drag or thrust directions. The application of the load was through strings tied to the model on its centerline. When the direction of the load needed to be switched, the entire apparatus was moved to the other side of the model. This method proved to be time consuming and not repeatable. The revised apparatus replaced the pulley with an inverted " $T$ " bracket. The bracket was attached to the model through a stiff rod and a rigid mount temporarily attached to the aftbody exit. The rigid rod and the " $\mathrm{T}$ " bracket allowed the application of the calibration load in both the drag and thrust directions without having to move or alter the apparatus. This method proved to be repeatable and quicker. The apparatus can be seen in Fig. 4-1. The calibration consisted of 7 points. The order of the loading was from negative axial force (net drag) to positive axial 
force (net thrust).The final calibration used for the data presented later is shown in Fig. 4-2. The average absolute value of the residuals is $0.14 \mathrm{lbs}$.

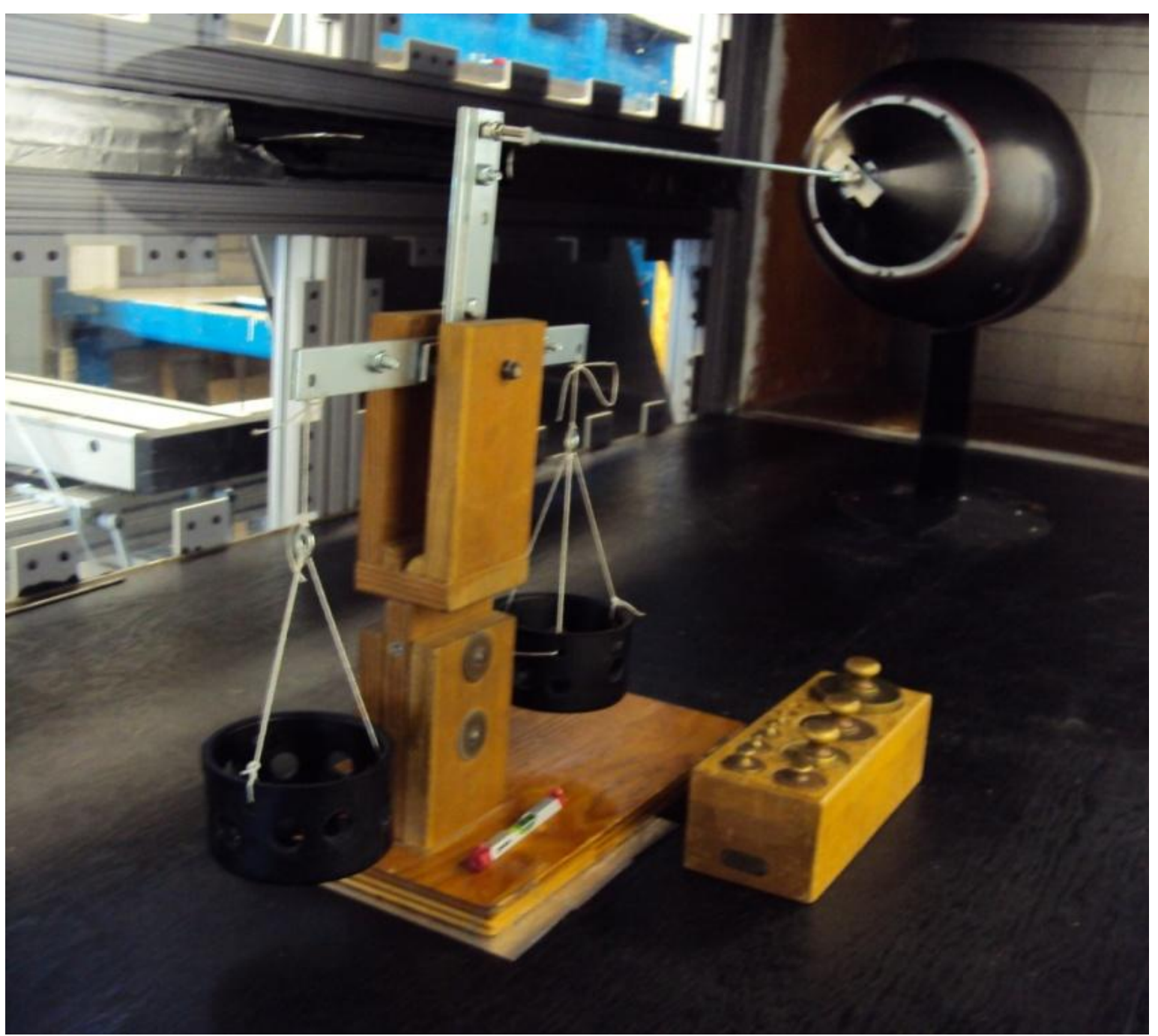

Figure 4-1. Apparatus used to calibrate the axial force load cell. 


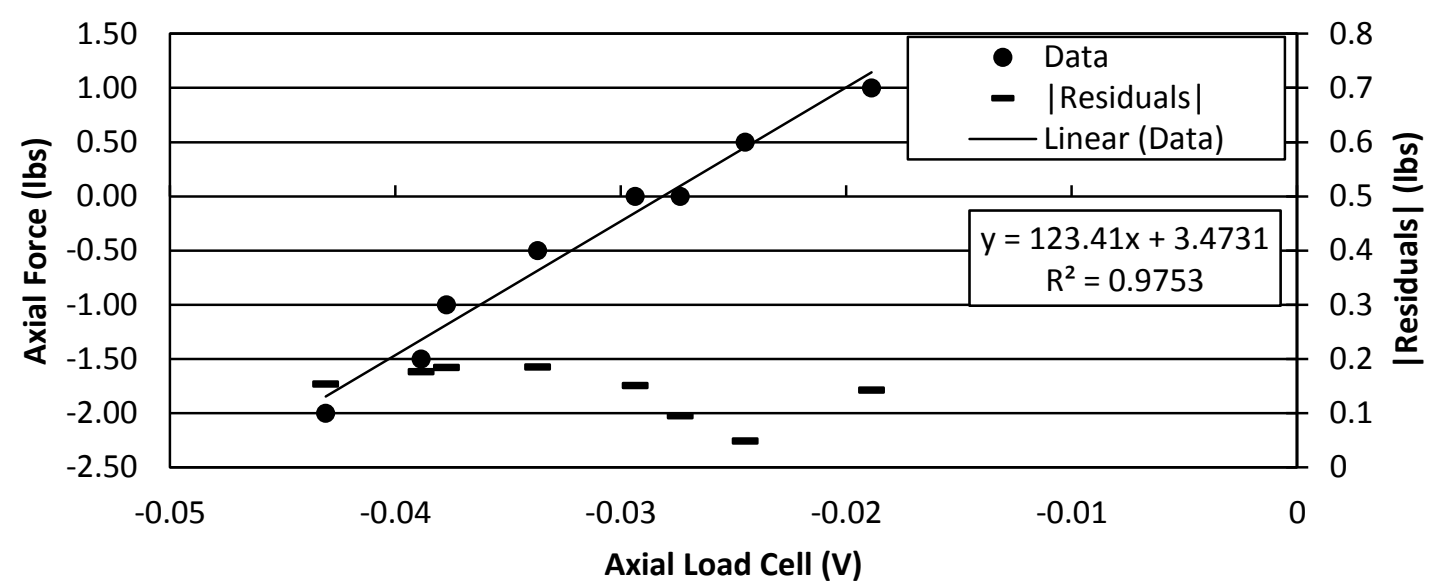

Figure 4-2. Calibration curve for axial force.

\subsubsection{FAN THRUST CALIBRATION}

The apparatus used for the thrust calibration is the same as that used for the axial force calibration except the force is applied in one direction only. Instead of applying force to a mount that is attached to the aftbody, the force is transmitted directly to the back of the fan motor. The calibration apparatus applies a force in the positive axial direction only. The apparatus can be seen in Fig. 4-3. The calibration curve can be seen in Fig. 4-4. The calibration is for the sum of the two load cells that measure fan thrust. The absolute values of the residuals for the calibration curve are also shown in this figure. The average absolute value of the residual is the $0.08 \mathrm{lbs}$. 


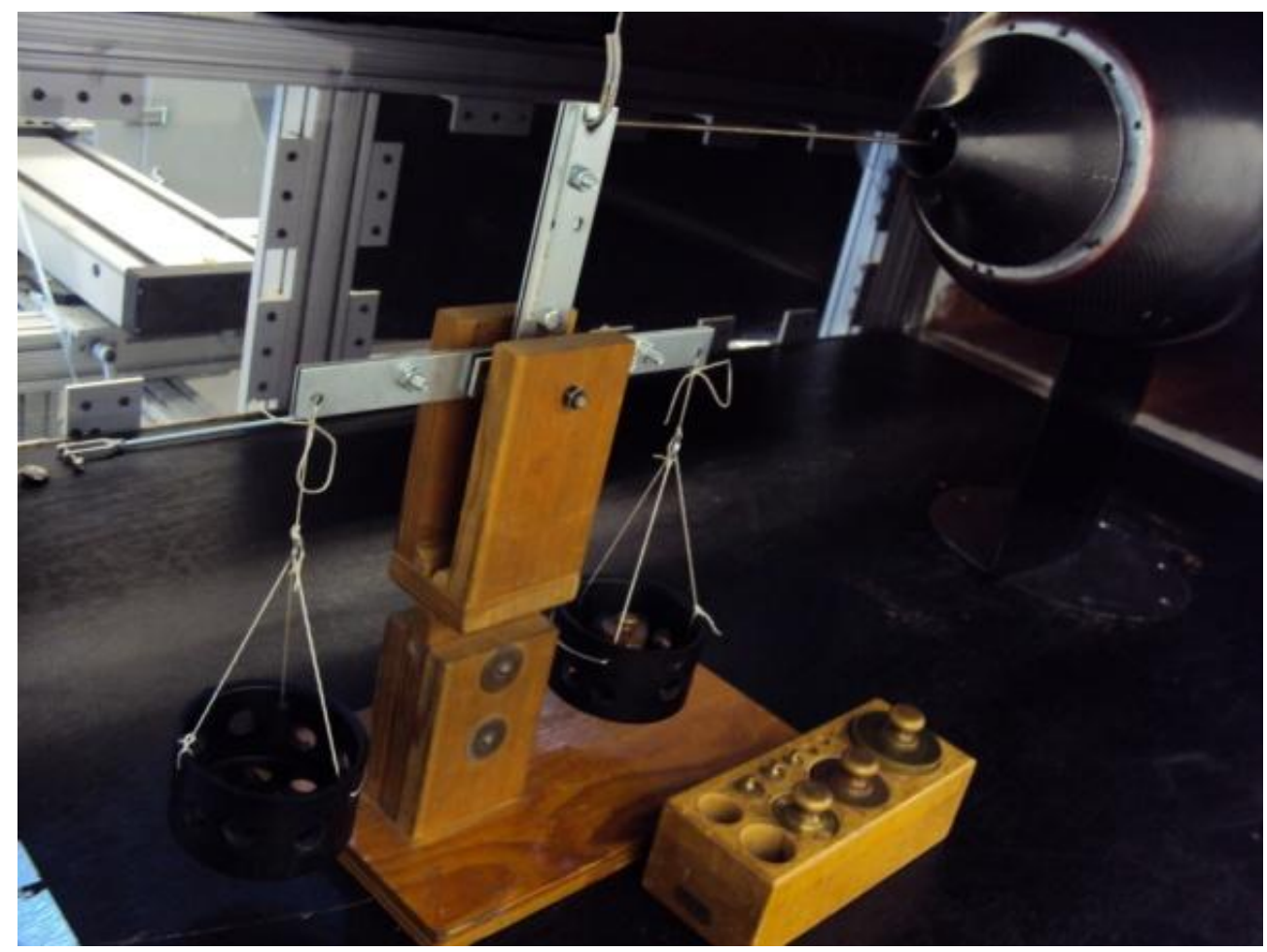

Figure 4-3. Apparatus used to calibrate the fan thrust load cells.

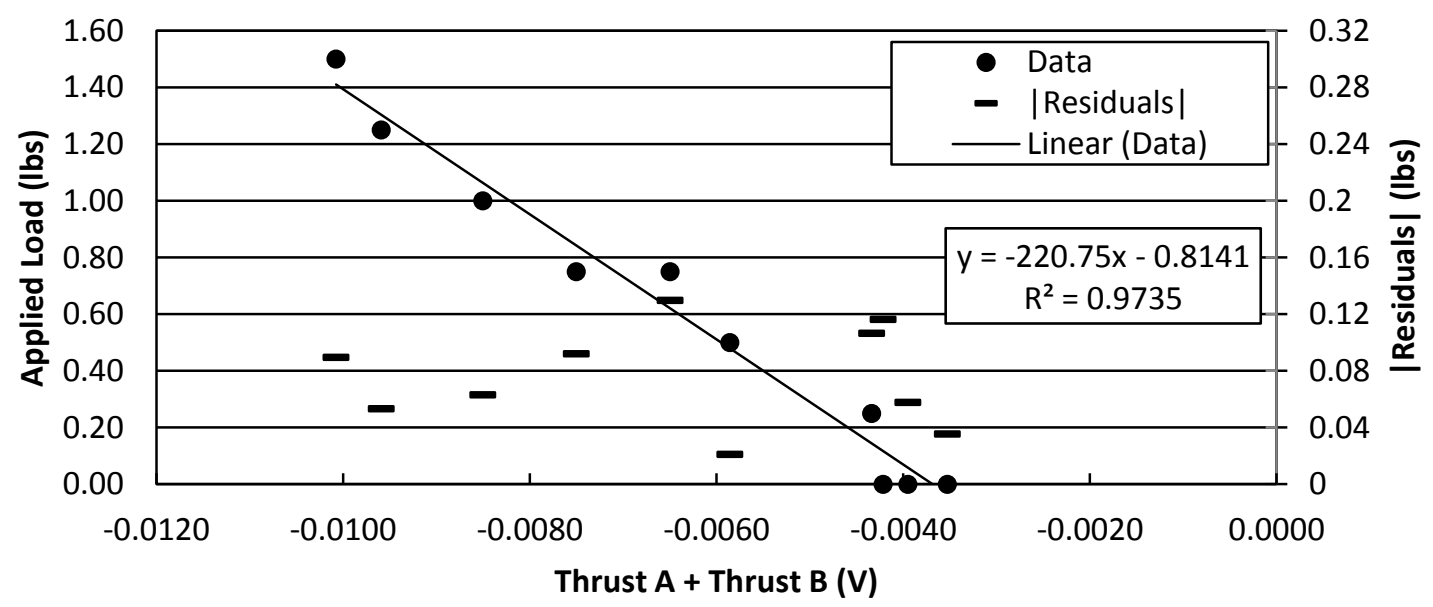

Figure 4-4. Calibration curve for the fan thrust load cells used for all data reduction.

\subsubsection{Fan Torque Calibration}

The apparatus to calibrate the fan torque is a unique piece of equipment. It can be seen in Fig. 4-5. The weight is placed in the black bucket and this weight is supported by two strings. These strings route around the 
pulleys and are then fastened tangentially to the outer body of the fan. The calibration weights produce a moment about the fan axis. This moment is then counter acted by the loads cells.

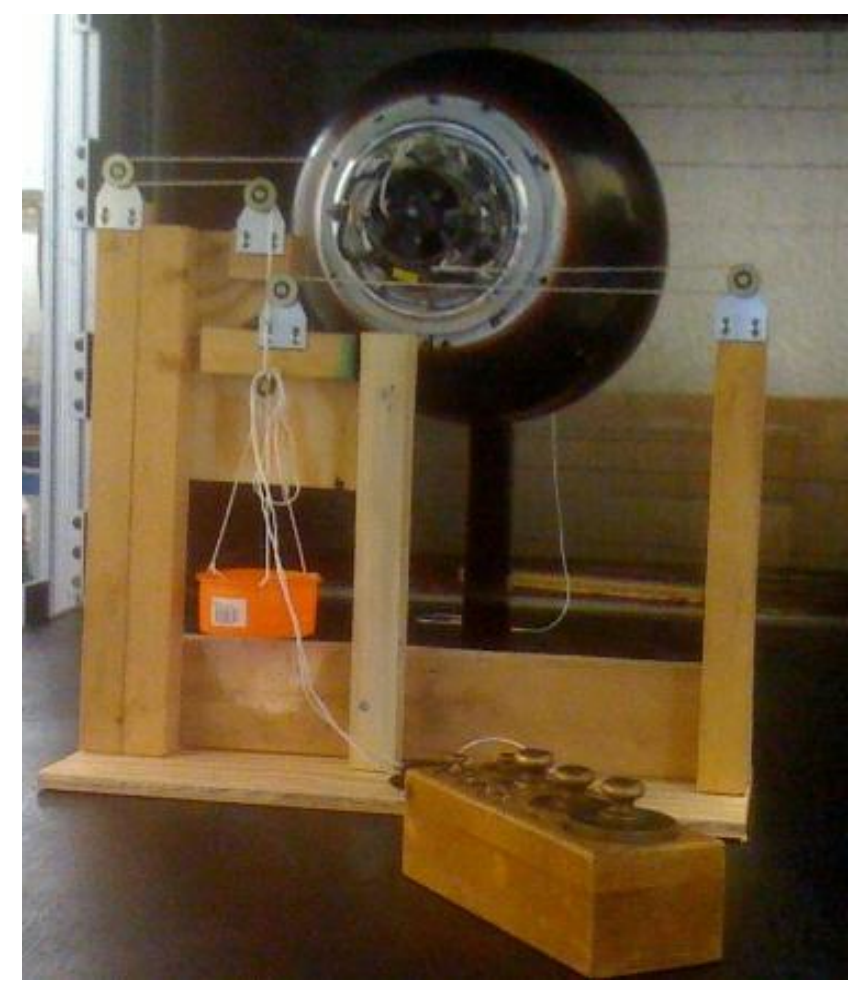

Figure 4-5. The apparatus used to calibrate the torque load cells.

The torque calibration process was challenging to make repeatable because of the friction in the many pulleys of the apparatus. To overcome this challenge, the pulleys were well lubricated and every time a new weight was placed in the weight bucket, the fan assembly was manually twisted at the same time the weight bucket was slowly pulled downward. This was to allow the pulleys to move slightly to overcoming any static friction. When the manual movement was released, the system would settle to its natural state. Completing this process during the calibration resulted in a repeatable calibration curve. This process was thought to be acceptable because, during actual testing, the fan and aerodynamic pressures would cause the entire model to vibrate. This vibration would serve the same purpose to overcome the static friction in the bearings as the manual movement of the device during calibration. The calibration curve obtained from this procedure can be seen in Fig. 4-6. This is a quadratic curve because it fits the data better than a linear curve. With the linear curve fit, the average absolute value of the residuals is 0.18 in-lbs, while with the quadratic curve, this was reduced to 0.066 in-lbs. 


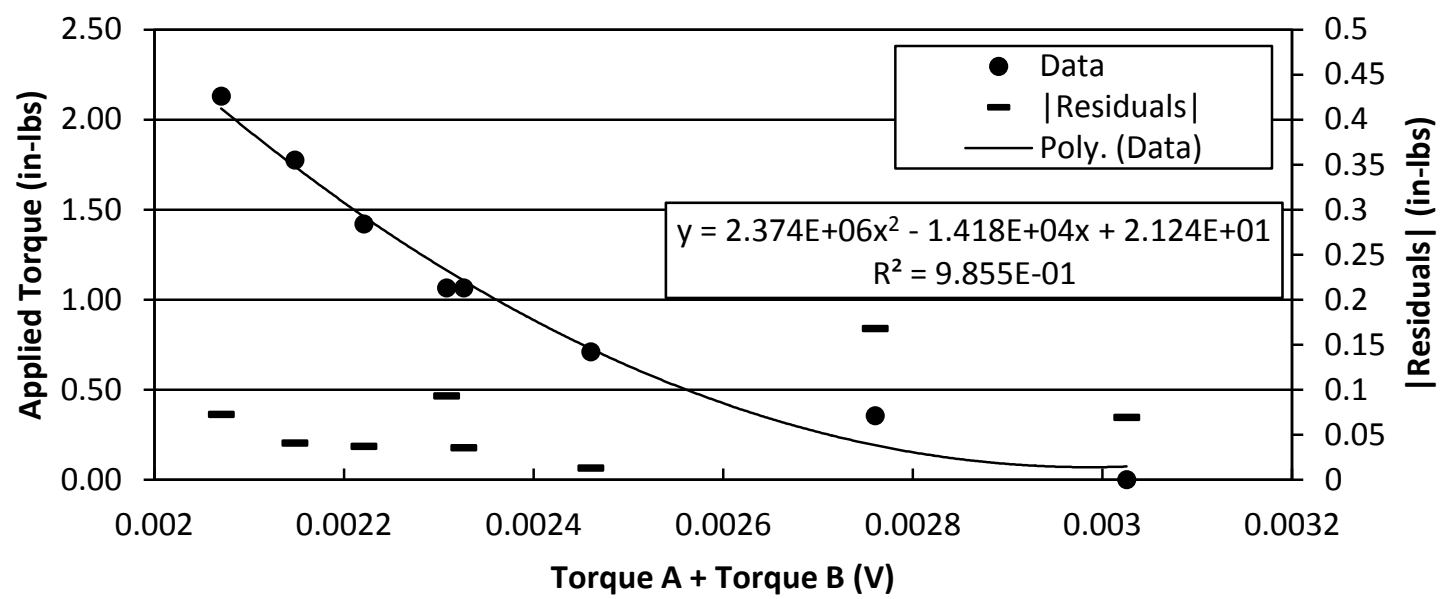

Figure 4-6. Calibration curve of the fan torque used for all data reduction.

\subsubsection{Slot Gap Calibration}

The slot gap was the secondary parameters of the test matrix. As mentioned earlier, it was measured with a simple spring-loaded linear potentiometer. To calibrate a potentiometer output voltage to an actual gap opening, the slot was set at various distances and the corresponding potentiometer voltage was recorded. For each slot opening distance, the actual size of the slot was measured with digital calipers at the top, bottom, and side of the model. These three measurements were then averaged to obtain the actual slot gap. The calipers are accurate to the nearest 0.001". The calibration curve can be seen in Fig. 4-7. The average absolute value of the residual for this calibration is 0.0026 ". While comparing the slot gap measurements at the top, bottom, and side of the model, it was evident that for each slot gap opening, the bottom measurements were consistently 0.015 " larger than the top measurements. This was deemed acceptable. With the addition of the cusp, the same calibration curve was used except it was offset by the thickness of the cusp, which was 0.188 ". 


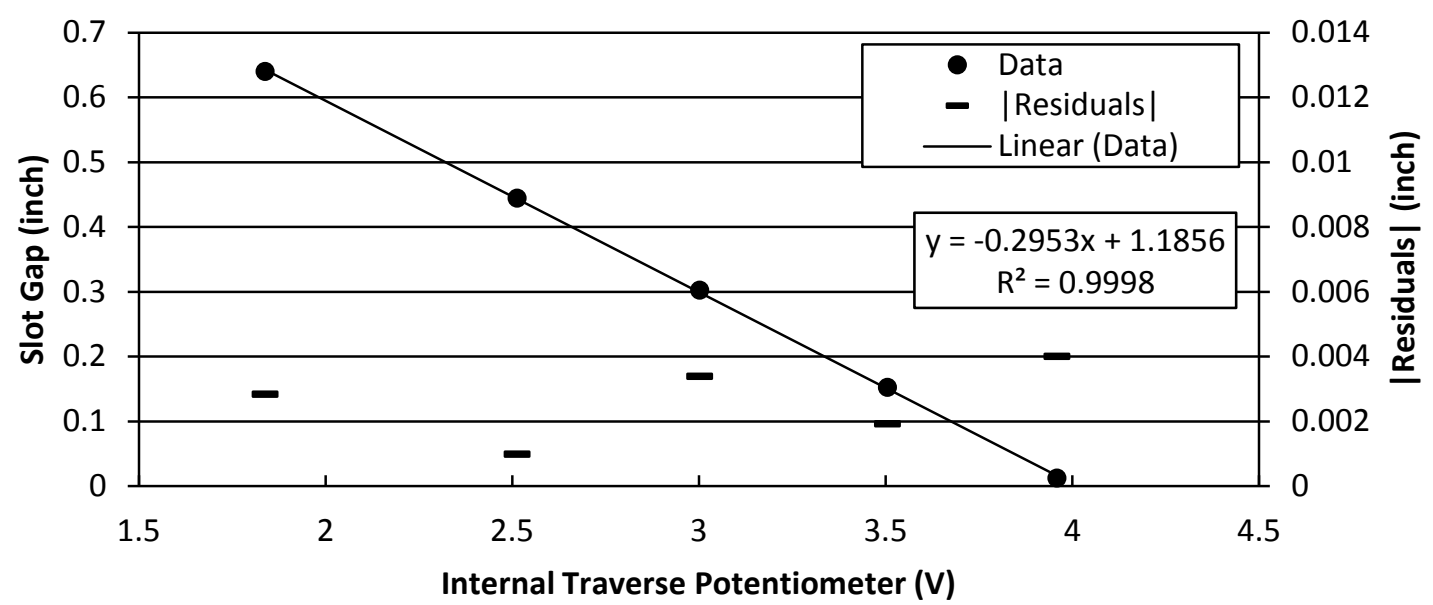

Figure 4-7. Calibration curve of slot gap that was used for all data without a cusp.

\subsubsection{Power Supply Voltage and Current Calibration}

The output channels on the fan power supply were utilized to measure the current and voltage that eventually make it to the fan. These two $0-5 \mathrm{~V}$ analog outputs correspond to $0-60 \mathrm{~V}$ and $0-50 \mathrm{~A}$ respectively. The default gain for these channels is therefore 12 for the voltage and 10 for the current. However, they were determined to be slightly inaccurate when checked against more accurate voltmeters and current-meters and even when compared to the display on the front of the unit. Subsequently, correlations were found to allow for accurate determinations of the delivered power. The power supply current and power supply voltage are corrected separately. The power supply current can be seen in Fig. 4-8. The average absolute value of the residuals is $0.048 \mathrm{~V}$.

The power supply voltage is a much simpler calibration. It requires a simple offset in the measured value of $-0.21 \mathrm{~V}$. This offset is believed to be due to aliasing errors in the sampling of the signal, as the signal was quite noisy, and the noise increased with power supply output. This offset is the average offset seen during the calibration, as the measured offset ranged from $0.164-0.314 \mathrm{~V}$. This variation of $0.15 \mathrm{~V}$ was used as the estimate of the error on the channel instead of the average absolute value of the residuals. 


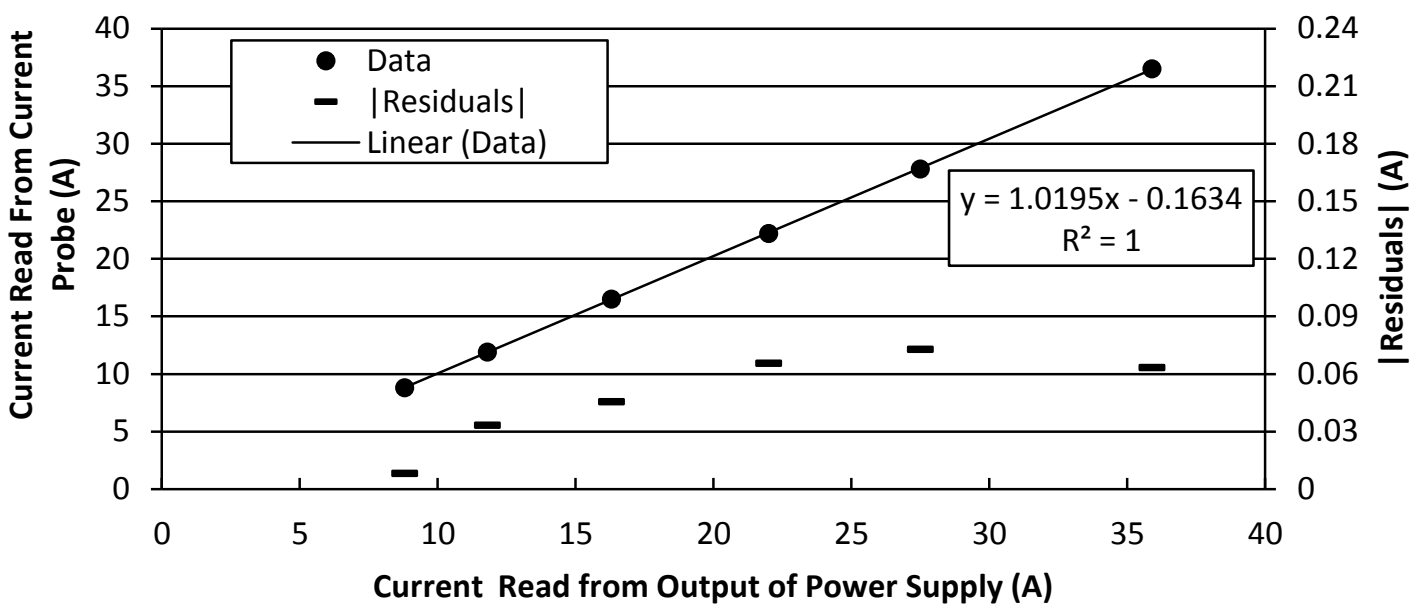

Figure 4-8. Power supply current calibration curve used fror all data.

\subsection{CORRECTIONS}

\subsubsection{Wind TunNel BuOYanCy CORRECTION}

The wind tunnel static and dynamic pressure varies in the direction of flow in the tunnel. As was mentioned earlier, this is due to the test section area being constant while the boundary layers grow in height. This growth of the boundary layers causes the streamlines of the freestream flow to converge and not be parallel. As taught in any aerodynamics class, the distance between steady streamlines indicates the relative change in flow speed. If streamlines are spreading apart, in general, the flow is slowing, and vice versa. For the wind tunnel, the converging streamlines indicates an acceleration of the flow outside of the boundary layers. Since nothing is adding energy to the flow, the total energy of the flow must stay the same even as the flow is accelerating. Since nothing is adding heat to the flow, the total energy can be expressed in termed of its total pressure, which is a local property. Since the local velocity is increasing in the streamwise direction, the local dynamic pressure is increasing, and therefore the local static pressure must be decreasing.

In reality, the total pressure is not constant as the flow travels down the tunnel. The total pressure decreases due to friction with the walls of the tunnel. However, the dynamic pressure still increases and the static pressure still decreases as the flow moves down the tunnel. For this to occur, the total pressure loss must be at a lower rate than the static pressure decrease, which is the case. 
The result of this axial static pressure gradient in the flow is an incremental force on the model. This force conceptually arises from the aft end of the model having a lower freestream static pressure than the front. This lower pressure at the aft side of the model tends to make the axial force more negative. Therefore, the measured model axial force is lower than it would be in free air conditions, or in a tunnel with no static pressure gradient. The amount of this force increment is given by Eq. 4.1. ${ }^{19}$ To evaluate this equation, the static pressure was measured in the empty tunnel with a pitot static probe at four locations in the tunnel starting at the nominal location of the nose of the model. The pressure measured at these locations is shown in Fig. 4-9. The change in static pressure was then calculated between the measurement locations. The four measurement locations only extend about 24 " from the nose of the model due to the limitation of the range of the traverse used, and therefore, did not reach to the aft end of the model. To approximate the static pressure all the way to the aft end of the model, it was assumed that the static pressure gradient would remain constant from the last measured value until the end of the model. This measured and approximated pressure gradient, as well as the model cross sectional areas at the measurement locations was then numerically integrated to obtain the axial force increment on the model. To correct for this pressure gradient effect, this increment will be added to the measured net axial force. The average measured pressure gradient is -0.0585 $\mathrm{psf} / \mathrm{ft}$.

$$
\Delta F_{a, B}=\int_{0}^{l} S(x) \frac{\Delta p_{\infty}}{\Delta x} d x=0.138 l b s
$$

This axial static pressure gradient is similar to the vertical static pressure gradient that exists in static fluids of much larger densities. In fluids such as a large tank of water, the static pressure is greater at the bottom than at the surface. This change in static pressure causes items in the fluid to experience an upward force. This is commonly referred to as buoyancy. Because of the similarity between the force exerted on a model due to the wind tunnel static pressure gradient and the upward buoyancy force in a fluid, the force on the model is often called a buoyancy force and the correction for it is referred to as the buoyancy correction. 

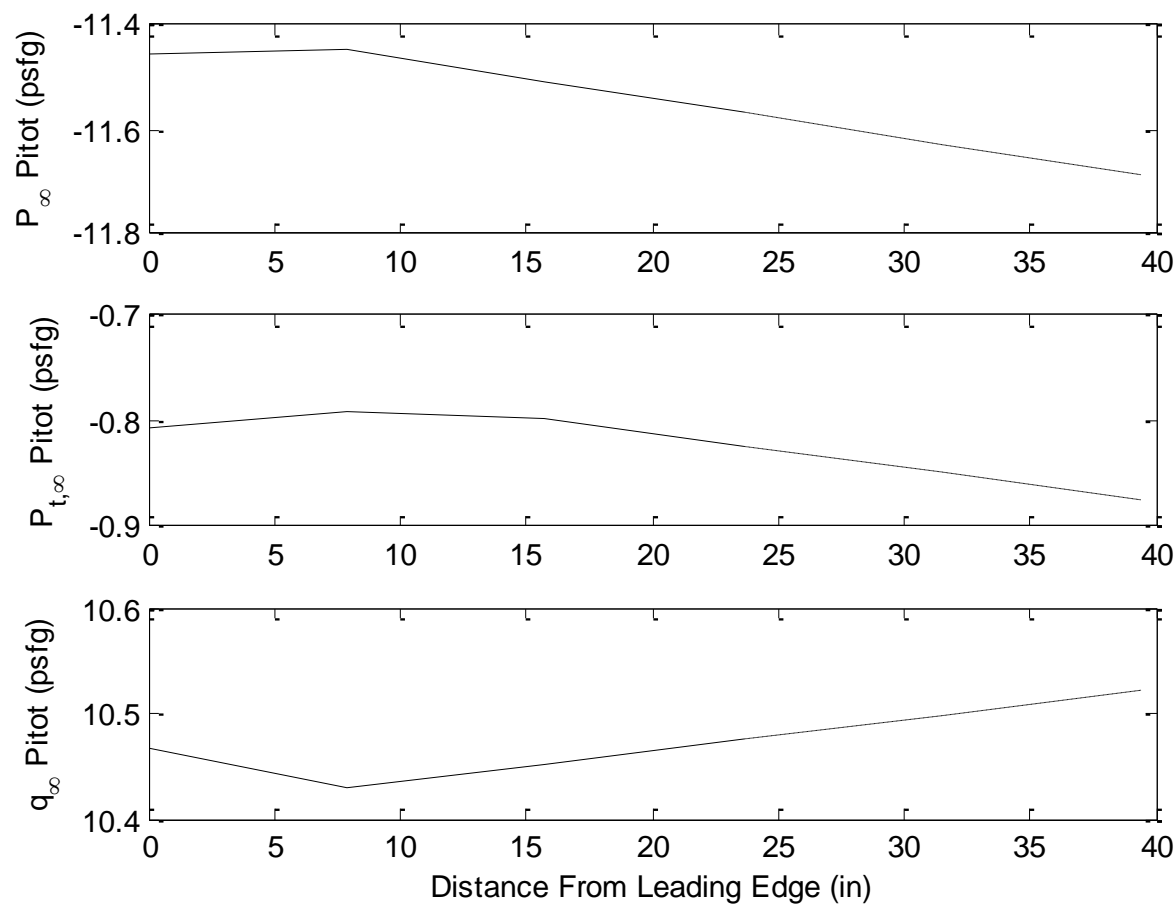

Figure 4-9. Wind tunnel axial variation of static pressure, total pressure, and dynamic pressure. The dashed lines are estimates based on an assumption of a constant pressure gradient after the last measured point.

\subsubsection{Wind Tunnel Static, Dynamic, \& Total Pressure Corrections}

In general, the true static, dynamic, and total pressure in the wind tunnel is difficult to obtain near a model because the model will affect any measurements in its vicinity. So to get accurate measurements of these three parameters near the model, they are measured upstream of the model and a correlation is found between the measured upstream parameters and their downstream counterparts. Total pressure is measured by a series of wall static pressure ports just behind the flow straightener in the tunnel inlet. The cross sectional area of the tunnel in this location is large, and the velocity is very small, so therefore, the static pressure is a good approximation of the total pressure of the flow in the test section. The total pressure of the flow cannot be assumed equal to the atmospheric pressure due to the pressure losses experienced when traveling through the flow straightener. To measure the static pressure upstream of the test section, the wall static pressure is measured at the exit of the contraction at various wall locations. In this area, the cross sectional area of the tunnel is essentially the same as the cross section of the test section, so the static pressure measured in this location is essentially the same as the static pressure in the test section. However, as mentioned in the previous section, the static and dynamic pressures change axially through the 
tunnel. Therefore, even though the static pressure and total pressure are known at the end of the contraction, the amount of change between this location and the location of the model must be measured.

Using the data collected in for the evaluation of the buoyancy correction, the static pressure at the location of the model center was correlated to the static pressure as measured from the upstream static pressure ring at the end of the contraction. This was also completed for the total pressure. Then a similar correlation was found for the dynamic pressure using the static and total upstream pressure measurements. The correction coefficients can be seen in Table 4-1. It is worth noting that these coefficients are valid for this location in the test section only, for the tunnel configuration in which they were measured, and for the freestream speed for which they were measured only. Any change to any of these parameters, and the coefficients would need to be re-measured.

Table 4-1. Summary of static, dynamic, and total pressure correction factors that were used for all analyses.

\begin{tabular}{|c|c|c|}
\hline Parameter & Correction Factor & Use Equations \\
\hline Static Pressure & 1.0332 & $\mathrm{P}_{\infty}=\mathrm{C}_{\mathrm{p}, \mathrm{s}} \mathrm{P}_{\infty, \mathrm{u}}$ \\
\hline Dynamic Pressure & 1.0296 & $\mathrm{q}_{\mathrm{pc}}=\mathrm{C}_{\mathrm{q}} \mathrm{q}_{\infty, \mathrm{u}}$ \\
\hline Total Pressure & 1.0880 & $\mathrm{P}_{\mathrm{t}, \infty}=\mathrm{C}_{\mathrm{p}, \mathrm{t}} \mathrm{P}_{\mathrm{t}, \infty, \mathrm{u}}$ \\
\hline
\end{tabular}

\subsubsection{Solid BLOCKAge CorRections}

In a free-air condition, as a body flows through the still air, the air will be displaced away from the body as it flows around the model. When testing a model in a wind tunnel, the walls constrict the flow around the model. This constriction results in the flow having to accelerate around the model in a manner that it would not have to if the model were in unrestricted airflow. This acceleration of the flow causes the model to experience a higher speed flow than is calculated from the freestream dynamic pressure. This higher tunnel velocity can be calculated using Eq. 4.2. ${ }^{19}$ This equation has its roots in potential flow but it has been experimentally validated for many model shapes and wind tunnel configurations. In it, $\mathrm{C}$ is the area of the test section, $\mathrm{V}_{\mathrm{B}}$ is the volume of the body under test, and $\mathrm{K}_{3}$ and $\tau$ are constants for the type of shape and type of wind tunnel, respectively.

For the current test, the increment of velocity due to the solid blockage of the model is calculated to be $5.0 \%$ above the freestream velocity. This uses the effective tunnel area instead of the geometric tunnel area. The 
effective tunnel area is the area outside of the wall boundary layers. The boundary layer height was measured in a previous effort to be very close to 1" high at this area of the tunnel. This results in an effective area of the tunnel of $9.94 \mathrm{ft}^{2}$, or $17 \%$ less area than the geometric tunnel area. The calculation of the model volume takes into account the volume of the fairing as well as the volume of the model. The combined model and fairing volume is $1.93 \mathrm{ft}^{3}$. The constant $\mathrm{K}_{3}$ is equal to 1.02 and $\tau$ is equal to $0.79 .^{19}$

$$
\varepsilon_{s b}=\frac{\Delta V}{V_{\infty}}=\frac{K_{3} \tau V_{B}}{C^{3 / 2}}=0.050
$$

\subsubsection{WAKe BLOCKage CORRECTIONS}

Wake blockage is a distinctly different blockage effect. It is a velocity increment due to the effect of the walls on the wake of the model. If the model were in free air conditions, the flow outside of the wake would be able to expand around the wake, but since the walls are present, the freestream flow is forced to accelerate around the wake of the model. This results in the pressure around the wake being lower than they would be in an unconstrained flow. Naturally, the magnitude of this effect is heavily dependent on the shape and size of the wake. The traditional equation for correcting for the wake blockage is given in Eq. 4.3. ${ }^{19}$ It is generally used for non-separated wakes of 2D and finite span wings where the wake deflects due to the lift produced. This deflected wake can be large depending on the amount of lift produced. For separated wakes, a method by Maskell is used. ${ }^{21}$ This method is summarized in Eq. 4.4 .

$$
\begin{gathered}
{\left[\frac{q_{c}}{q_{u}}\right]_{\text {unseparated }}=\left(1+\varepsilon_{w b, u s}\right)^{2}=\left(1+\frac{C_{d u} S}{4 C}\right)^{2}=\left(1+0.025 * C_{a, u}\right)^{2}} \\
{\left[\frac{q_{c}}{q_{u}}\right]_{\text {separated }}=1+\theta \frac{C_{a, u} S}{C}}
\end{gathered}
$$

For the non-separated case, the velocity increment is approximately $2.5 \%$ of the uncorrected axial force coefficient. Since the maximum axial force coefficient is estimated to be about 0.05 , the velocity increment for a non-separated case would never exceed $0.1 \%$ of the velocity. This small magnitude of velocity increment is below the accuracy of the tunnel freestream velocity calculation and is therefore not included in the data reduction process. 
For the separated case, Maskell stated that a good safe value for $\theta$ in separated wakes is $5 / 2$. This value was derived for plat plates normal to the flow direction. For all cases presented by Maskell, $\theta$ ranged from 0.96 to 2.77 , of which $5 / 2$ falls toward the higher end. For the highest value of $\theta$ that could be expected, 2.77 , and the highest estimated value of the axial force coefficient, $\mathrm{C}_{\mathrm{a}, \mathrm{u}}$, of 0.05 , this results in a dynamic pressure increment of $1.39 \%$ of the uncorrected axial force coefficient. This results in a velocity increment of $0.69 \%$ of the wind tunnel freestream speed. Because of this expected small value of this correction factor, this correction was also not included in the data reduction process. Besides the reason just stated, the applicability of the method to the current test case is questionable. The justification for Maskell's method used flat plates normal to the flow and wing sections, both 2D and of finite span, at very high angles of attach where they were severely stalled. Both of these cases have separated wakes that extend outside of the cross sectional area of the model. The similarity of these cases to the axisymmetric pseudo-streamlined body of the NGP is questionable at best. Maskell himself questioned the applicability of his method for even objects as streamlined as a cylinder or sphere.

A third justification for excluding the wake blockage corrections is the necessity, and limited ability, to differentiate between what is considered to be a separated wake and what is a non-separated wake. For most of the data points, the wake is partially separated. This partial separation does not fit neatly into either of the two categories. Presumably, Maskell's method could be used for these cases, but the value of $\theta$ would need to be evaluated for each case. For this reason, it is convenient that the correction is estimated to be very small, as explained above, and this ambiguity is avoided.

\subsubsection{LoAd Cell Zero ShIFT CORRECTIONS}

All load cells are subject to a small amount of drift over time. This is usually seen in terms of the shift of the "zero" point in the load cell. The "zero point" is simply the output of the load cell with no load applied. The load cells are simply Wheatstone bridges bonded to a substrate. This drift is due to two sources. The first source is due to the changes in the temperature of the actual Wheatstone bridge, which results in the resistivity of the individual wires in the load cells changing. A hotter wire will have more resistivity and a higher resistance. The second is due to the relative difference in temperature between the substrate and the Wheatstone bridge. The temperature change will cause the substrate to expand, while the bridge does not expand by the exact same amount. This effect is present even if the bridge and substrate are at the same temperature, and it is more pronounced if they are not. This 
expansion of the substrate is seen as stress in the bridge even thought the load cell has no load on it. Both of these effects cause the zero point of the load cell to drift.

To combat these sources of drift, the Wheatstone bridge is matched with a substrate with a very similar CTE, or coefficient of thermal expansion, for a range of operating temperatures where the load cell is expected to operate. In addition, the substrate and bridge have electrically insulating but thermally conductive encapsulating material between, and surrounding, the bridge on the substrate. In addition, the wires of the Wheatstone bridges can be alloyed to have a very low resistivity change with temperature. Even with these measures, the thermal drift of load cells is usually the limiting factor on the accuracy of any load cell.

One way to minimize the drift in the measurements is to let the load cell and substrate reach an equilibrium temperature prior to using it. Anytime current is flowing through the load cell, the resistance of the bridge is heating the bridge and substrate. After a period of time, the heat generation will match the heat dissipation into the air and substrate, and a steady temperature will be reached. It is good practice to shield all load cells during calibration and during operation from any airflow to prevent this thermal equilibrium from being upset or altered in any way. This was done for the current test by enclosing the load cells in parts of the NGP that do not see appreciable airflow.

To compensate for the zero shift of the load cells from when the calibration was completed to when the data was actually being taken, a wind off zero, or WOZ, run was recorded with the wind tunnel off and the model fan off. The purpose of the WOZ run was to keep track of the drift of the five load cells. Then any drift can be compensated for in the data reduction process. The first step in reducing the data is to record the zero value during the calibration, this will be referred to as the $\mathrm{V}_{\mathrm{cz}}$, or calibration zero voltage. Then the WOZ reading is subtracted from this value to get the $\Delta \mathrm{V}_{\mathrm{zs}}$, or zero shift voltage increment. This can be seen in Eqs. 4.5 and 4.6. This method can be understood with the help of Fig. 4-10. The main assumption is that the slope of the calibration line remains the same when the zero shift occurs. The zero shift results in a change in the calculated load on the load cell. This additional load, either positive or negative, is equal to the difference in the y-intercepts of the two lines. Unfortunately, the y-intercept of the shifted line is not known, but the slope and the lateral shift are known. These two parameters are all that is required to account for the zero shifts of the load cells as given in Eq. 4.6.

$$
\Delta V_{z s}=V_{c Z}-V_{W O Z}
$$




$$
F_{2}=F_{1}+\Delta B=M\left(v+\Delta V_{z s}\right)+B_{1}
$$

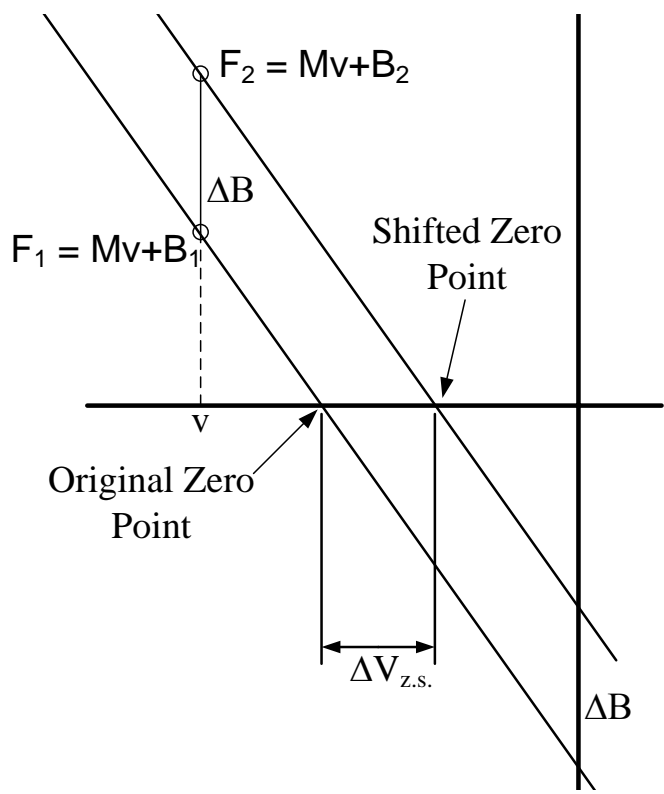

Figure 4-10. Diagram of the zero shift of the load cells.

\subsubsection{FAIRING INTERFERENCE CORRECTIONS}

The fairing that surrounds the strut is an important part of the experimental apparatus. It shields the strut, wiring, and tubing from the airflow for most of the distance from the tunnel floor to the model. The fairing does not touch the model or model strut. This does not mean that the fairing does not influence the flow around the model. The fairing actually induced a force on the model, and the model induces a force on the fairing, but since the fairing does not touch either the model or strut, the loads on the fairing are not important. What is important is the determination of the force that is induced on the model from the presence of the fairing. This force increment is found by placing a dummy fairing on the opposite side of the model as to mimic the original fairing. The model would then have twice the force induced on it by the fairing, and by keeping all test conditions the same with the dummy fairing as a normal test point, the effect of the dummy fairing can be isolated. The test point that was chosen for this comparison was with a slot gap nominally 0.5 " and the fan set at nominally 500 Watts. The increment for the fairing is $0.016 \mathrm{lbs}$. This small of an increment was believed to be of the same order of magnitude of the accuracy of the measurement, so it was not included in the data reduction process. The dummy fairing installed in the wind tunnel can be seen in Fig. 4-11. 


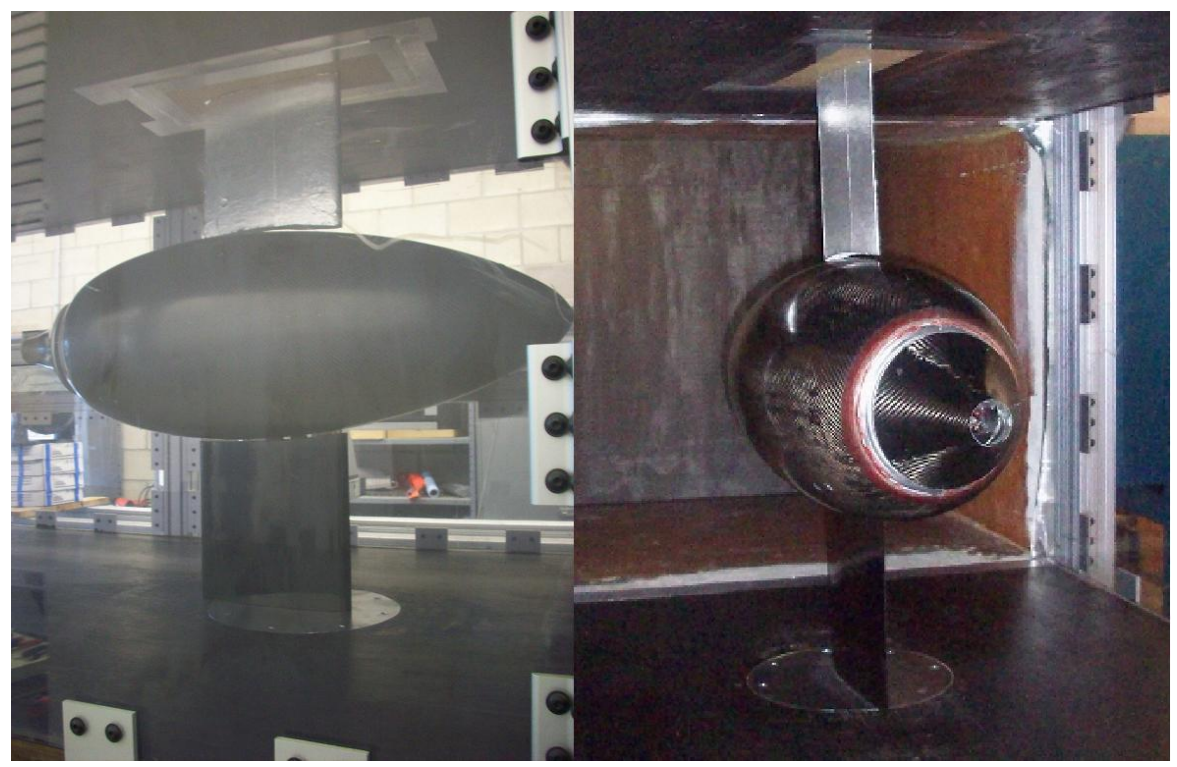

Figure 4-11. The silver dummy strut was used to measure the influence of the fairing on the measured axial force of the model.

\subsection{COMPLETE DATA REDUCTION PROCEDURE}

\subsubsection{FREESTREAM PROPERTIES}

The process for calculating the free stream properties starts with the calculation of the density according to Eq. 4.7. This is followed by the dynamic pressure from the total and static pressures of the pressure rings around the contraction from Eq. 4.8. This is followed by the freestream speed and Reynolds number according to Eq. 4.9 and Eq. 4.10.

$$
\begin{gathered}
\rho_{\infty}=\frac{P_{t, w}}{R T_{\infty, w}} \\
q_{\infty}=C_{q}\left(1+\varepsilon_{s b}\right)^{2}\left(P_{t_{\infty}}-P_{\infty}\right) \\
U_{\infty}=\sqrt{\frac{2 q_{\infty}}{\rho_{\infty}}} \\
R e_{L}=\frac{\rho_{\infty} U_{\infty} L}{\mu}
\end{gathered}
$$




\subsubsection{AXIAL FORCE}

The axial force on the model is a one of the primary outputs of the current work. The processing of the data for this parameter is the most complex of the all parameters measured during the testing. It was previously outlined in Section 4.2.5, but will be repeated here for completeness. The first step is to determine the zero shift of the load cell according to Eq. 4.11. This shift, along with the actual voltage read from the load cell is evaluated by the calibration equation in Eq. 4.12. The sign convention for this equation is positive axial force is forward, and negative axial force is in the negative direction. The axial force is then corrected for buoyancy. This correction is positive because it causes extra force in the negative direction.

$$
\begin{gathered}
\Delta V_{z s, A}=V_{c z, A}-V_{W O Z, A} \\
F_{A, u}=123.41\left(v_{A}+\Delta V_{z s, A}\right)+3.4731 \\
F_{A}=F_{A, u}+\Delta F_{A, B}=F_{A, u}+0.138
\end{gathered}
$$

\subsubsection{Pressure ForCe}

The equation that was derived for calculating the axial pressure force is given in Eq. 4.14. It was derived using Fig. 4-12. The derivation of this pressure drag equation is given in Appendix C. It is worth noting that the pressure drag calculation does not take into account any of the surface pressures inside the suction slot. These surface pressures in this area of the model are not resolved enough to be able to make an accurate estimate of the effects. The sign convention for this equation is consistent with the axial force sign convention. Negative values indicate a net pressure force coefficient in the negative direction, and vice versa. The reference area used is the frontal area of the model.

$$
C_{A, p}=\sum_{i=1}^{N-1}-\left(\frac{C_{p, i+1}+C_{p, i}}{2}\right)\left[\frac{\pi\left(r_{i+1}^{2}-r_{i}^{2}\right)}{A_{r e f}}\right]
$$




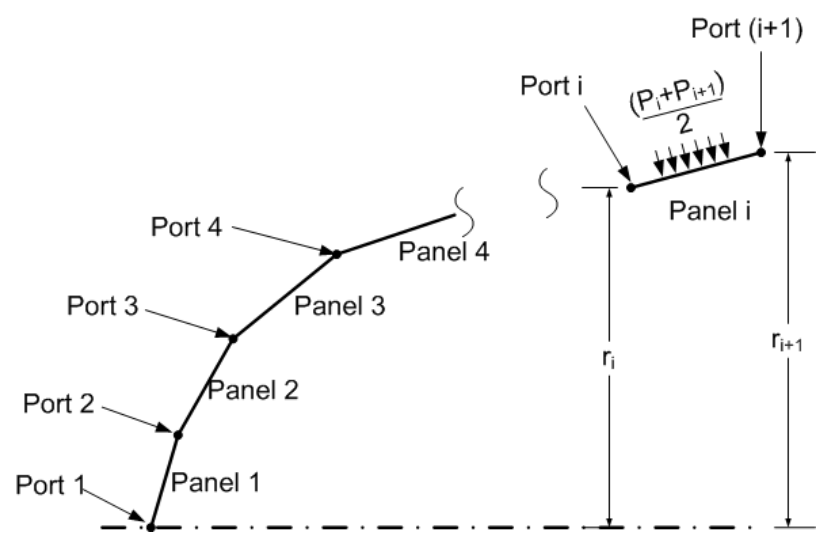

\section{Figure 4-12. Diagram of a generic surface pressure port configuration used to derive the axisymmetric pressure drag equation.}

The equation that was used for the calculation of the axial pressure force coefficient was validated to be correct with experimental pressure data for a sphere. The experimental data can be seen in Fig. 4-13 for the subcritical and supercritical cases. The subcritical Reynolds number is 162,000 with a stated pressure drag coefficient of -0.46 and a calculated coefficient of -0.45 . The supercritical Reynolds number is 435,000 with a stated drag coefficient of -0.082 and a calculated coefficient of -0.096 . The larger error for the supercritical case is attributed to the larger pressure gradients that are not well captured in the data compared to the relatively small pressure gradients that are adequately defined for the subcritical case.

The subcritical case is when the boundary layer has not yet transitioned to be turbulent before the pressure gradient becomes too adverse and the boundary layer has separated. This laminar separation is the defining characteristic for the subcritical case. The supercritical case in when the boundary layer has transitioned to turbulent prior to the boundary layer separation. This turbulent separation is what defines the supercritical case. The change from subcritical to supercritical happens very suddenly with increasing Reynolds number, and occurs approximately at a Reynolds number based on the sphere diameter of 385,000. 


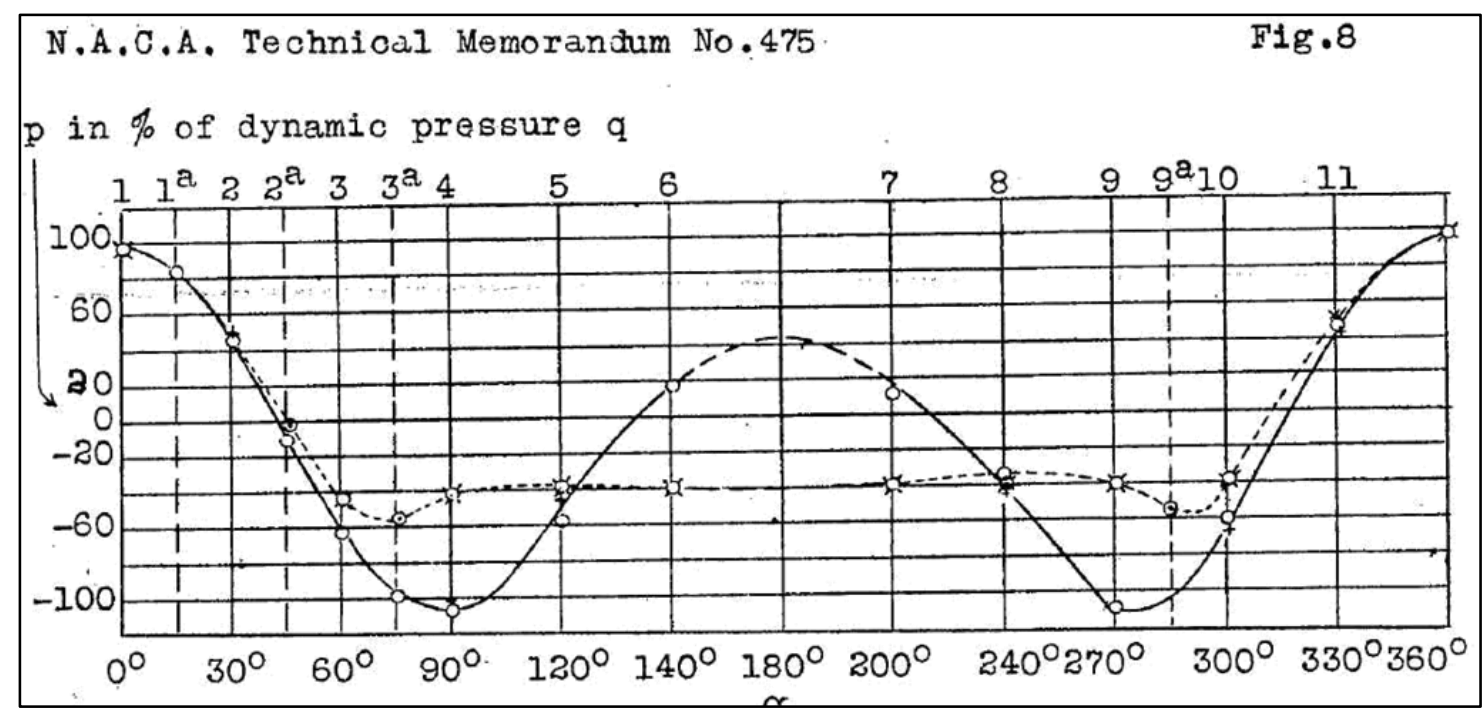

Figure 4-13. Subcritical (solid line) and supercritical (dashed line) surface pressure data of a sphere used to validate the equation derived to calculate axial pressure force. ${ }^{22}$

\subsubsection{Aftbody Exit Properties}

The properties at the aftbody exit are mainly used to calculate the suction mass flow rate. The exit density is calculated based on the local static pressure, as read from the pitot static probe, and the exit temperature of the flow, as measured by a thermocouple at the exit of the aftbody. The local static pressure is a gauge pressure, so it is added to the atmospheric pressure to achieve an absolute pressure measurement of the static pressure at the exit of the aftbody.

$$
\begin{gathered}
\rho_{e}=\frac{P_{s, e}+P_{t, w}}{R T_{e}} \\
q_{e}=P_{t, e}-P_{s, e} \\
\bar{U}_{e}=\sqrt{\frac{2 q_{e}}{\rho_{e}}}
\end{gathered}
$$

\subsubsection{SuCtion MASS Flow RATE}

The suction mass flow rate is calculated from a measured velocity at the exit of the slot, the density at the exit and the exit area. The calculated velocity is assumed the average velocity at the exit of the flow. 


$$
\dot{m}=\rho_{e} A_{e} \bar{U}_{e}
$$

\subsubsection{Power Supply Voltage ANd Current}

The voltage and current that leave the power supply are given by the following two equations.

$$
\begin{gathered}
V_{p s}=12 * v_{f v}-0.21 \\
I_{p s}=1.0195 * 10 * v_{f i}-0.1634
\end{gathered}
$$

\subsubsection{FAn Thrust AND TORQue}

The fan thrust is calculated by first calculating the zero shifts of the load cells. Then the output voltages of the two load cells are summed and then this sum is evaluated with the calibration equation. This is shown in Eq. 4.21 and Eq. 4.22. The same basic procedure is followed for the fan torque calculation, and is shown in Eq. 4.23 and Eq. 4.24 .

$$
\begin{gathered}
\Delta V_{z s, a f}=V_{c z, a f}-\left(V_{W O Z, a f 1}+V_{W O Z, a f 2}\right) \\
F_{a, f}=-220.75\left(v_{a f 1}+v_{a f 2}+\Delta V_{z s, a f}\right)-0.8141 \\
\Delta V_{z s, m f}=V_{c z, m f}-\left(V_{W o Z, m f 1}+V_{W O Z, m f 2}\right) \\
M_{A, f}=-2074.1\left(v_{m f 1}+v_{m f 2}+\Delta V_{z s, m f}\right)+6.0741
\end{gathered}
$$

\subsubsection{FAN AND MOTOR POWER}

The shaft power that enters the fan is given by Eq. 4.25. This equation accounts for the assumed

efficiencies of the wiring, ESC, and motor, as discussed in Section 3.2.13. The fan power is calculated from the fan torque and rotational speed as shown in equation. The fan efficiency is the ratio of the output power, the fan power, to the input power, the shaft power. This measure of fan efficiency is limited by the assumptions of the component efficiencies. These assumed efficiencies of the wiring, ESC, and fan motor effectively lower the input power to the fan, thus increasing the apparent fan efficiency. A "worst case" scenario is to assume that these items are 100\% efficient, which would give the lowest possible value for the fan efficiency. This was not done, and the component 
efficiencies of less than $100 \%$ were used, in order to give a better estimate of the real fan efficiency at all operating points.

$$
\begin{gathered}
P_{s}=0.855 V_{p s} I_{p s} \\
P_{f}=M_{A, f} \omega_{f} \\
\eta_{f}=\frac{P_{f}}{P_{s}}=\frac{M_{A, f} \omega_{f}}{0.855 V_{p s} I_{p s}}
\end{gathered}
$$

\subsubsection{NON-DimENSIONALIZATION OF DATA}

To be able to compare to the previous tests of the Goldschmied propulsor, the data will be nondimensionalized in a similar manner as the previous tests. The traditional coefficients of the axial force, surface pressure, and pressure force are shown in Eq. 4.28 through Eq. 4.30.

$$
\begin{gathered}
C_{A}=\frac{F_{A}}{q_{\infty} A_{r e f}} \\
C_{p}=\frac{p-P_{\infty}}{q_{\infty}} \\
C_{A, p}=\frac{F_{A, p}}{q_{\infty} A_{r e f}}
\end{gathered}
$$

The less common coefficients that will be used are the suction mass flow coefficient and the slot pressure difference coefficient.

$$
\begin{gathered}
C_{q}=\frac{\dot{m}}{\rho_{\infty} U_{\infty} A_{\text {ref }}} \\
\Delta C_{p, \text { slot }}=\frac{P_{\text {aft slot }}-P_{f w d \text { slot }}}{q_{\infty}}
\end{gathered}
$$




\subsubsection{Flight Power}

The power required to fly is central in the evaluation of the concept of the Goldschmied propulsor. The goal of this section is to show the relationship between the shaft power and the axial force for a conventional aircraft. This relationship will then be compared in Section 6.6 to the measured relationship of the NGP.

For a traditional aircraft, the power available to fly a given speed is shown in Eq. 4.33. This is related to the shaft power through the propulsive efficiency in Eq. 4.34. The axial force is the difference between the drag and thrust available. It is worth noting that the drag force is assumed to be the same for a conventional aircraft no matter if there is power being consumed or not. The thrust available is replaced by the power available, and eventually, the shaft power and propulsive efficiency. The resulting Eq. 4.37 gives the axial force coefficient as a function of the shaft power of an aircraft. The reader is reminded that this is for a traditional aircraft configuration. For the NGP to show a benefit, the axial force coefficient for a given shaft power must be higher than given by this equation. This comparison determines the benefit of the NGP.

$$
\begin{gathered}
P_{a v}=T_{a v} U_{\infty} \\
T_{a v}=\frac{P_{a v}}{U_{\infty}}=\frac{\eta_{p} P_{s}}{U_{\infty}} \\
C_{A}=\frac{F_{A}}{q_{\infty} A_{r e f}}=\frac{T_{a v}-D}{q_{\infty} A_{r e f}}=\frac{T_{a v}}{q_{\infty} A_{r e f}}-\frac{D}{q_{\infty} A_{r e f}} \\
C_{A}=\frac{P_{a v}}{q_{\infty} A_{r e f} U_{\infty}}-\frac{D}{q_{\infty} A_{r e f}} \\
C_{A}=\frac{\eta_{p} P_{s}}{q_{\infty} A_{r e f} U_{\infty}}-C_{D}
\end{gathered}
$$

The drag terms in Eq. 4.35 through Eq. 4.37 might seem a little ambiguous at first glance, but these terms are simply the drag of the aircraft at a given flight speed. For a traditional aircraft, its drag is thought to be the same whether or not the propulsors are consuming power or not. The drag is simply a summation of the parasite drag, wave drag, and the induced drag, or the zero-lift drag, and the drag due to lift (however one chooses to partition the drag terms). For the current comparison, an axisymmetric aircraft with no wings at a zero degree angle of attack at low Mach and Reynolds numbers is chosen to be compared to the axisymmetric NGP. Therefore, there is no induced 
drag or wave drag for the conventional aircraft design. This eliminates the need to include assumptions like the weight (lift) of the aircraft, the aerodynamic efficiency, aspect ratio, etc.

In the above analysis, the available power is equivalent to the shaft power because this is the only type of power available, and because it uses shaft power, the efficiency is propulsive efficiency and not overall efficiency. This is intentionally done to remove the need to assume a thermal efficiency for the conventional aircraft. In the case of the NGP experimental data, the thermal efficiency is replaced by the electrical efficiency of the motor and wires. The propulsive efficiency for a conventional aircraft is given by Eq. $4.38 .^{23}$ This shows that very efficient aircraft have an exit velocity that is very close to the freestream velocity. The problem with this is that these same aircraft produce very little thrust as shown by Eq. 4.39. According to Raymer ${ }^{23}$, turbojets can have a propulsive efficiency of $50 \%$ or lower because they have an exit speed that is three times the freestream speed. Also from Raymer ${ }^{23}$, propellers can have propulsive efficiency values around $80 \%$ because the exit speed behind typical propellers are only 1.5 times the freestream speed. These propeller aircraft must accelerate a much larger mass of air, by having a large cross-sectional area, to produce equivalent thrust as a turbojet.

$$
\begin{gathered}
\eta_{p}=\frac{2}{\left(\frac{U_{e}}{U_{\infty}}+1\right)} \\
T=\rho A U_{e}\left(U_{e}-U_{\infty}\right)
\end{gathered}
$$

\subsubsection{ERROR ANALYSIS}

An error analysis of the random error of the data is completed for every data point of every parameter. The error analysis uses the standard deviation of the mean as the estimate for the error and a confidence interval of $95 \%$. The error propagation uses the "most probable" error in opposed to the maximum error. The most probable error is the square root of the sum of the squared independent sources of error instead of simply summing the sources of error in the maximum error method. Using this method, the random error estimates calculated are very small. This is due to the large number of measurements that were taken at each point in the test matrix. For the pressure data measured, 3000 samples were taken, while for the data taken by the National Instruments system, 1000 samples were taken at each data point. The Measurement Computing system recorded the temperature data and it took 100 samples at each data point. The number of samples of fan speed data could not be directly controlled. It was 
measured at a frequency of $10 \mathrm{~Hz}$. These error estimates were originally going to be included on all figures, but due to their small magnitude, they detracted from the clarity of the presentation. Therefore, they were left off all plots. 


\section{EXPERIMENTAL PROCEDURE}

As stated in Section 3.2.2, the target Reynolds number for this test is 3.2 million. To try to achieve this high of a Reynolds number, the tunnel was going to be run at its maximum continuous speed of $98.4 \mathrm{ft} / \mathrm{s}(30 \mathrm{~m} / \mathrm{s})$. This would have resulted in a test Reynolds number of 2 million.

In fact, a complete test matrix of data was taken at this tunnel speed with the $500 \mathrm{~W}$ fan motor installed. In addition to the initial test matrix, three additional test matrices were also completed. These additional test matrices tested with different cusp geometries. Due to tunnel time restrictions, the flow visualization was left until the end of the testing to ensure that there was time to take all points in the test matrix, and whatever time was left would be dedicated to flow visualizations. The initial smoke flow visualization exhibited what was believed to be attached flow. Once the tuft flow visualizations were complete, it was evident that the flow was not fully attached for any of the test conditions in the initial test matrix. Nevertheless, before this absolutely critical evaluation was made, the initial data set and test report were completed and published by Thomason. ${ }^{20}$ The data presented by Thomason is now invalidated by the data presented here.

In an attempt to create fully attached flow with a tunnel speed of $98 \mathrm{ft} / \mathrm{s}$, the $700 \mathrm{~W}$ fan was installed and some of the test points were repeated. This still did not show fully attached flow on the aftbody. The decision was made to lower the tunnel speed until fully attached flow was evident on the aftbody. Steady fully attached flow occurred at a tunnel speed of $66 \mathrm{ft} / \mathrm{s}(20 \mathrm{~m} / \mathrm{s})$. Therefore, the complete test matrix of data was taken at this lower tunnel speed with the simple slot gap, and the test matrix was repeated with one of the cusp geometries as well.

\subsection{TEST MATRIX}

The original test matrix had 60 distinct data points. The main test variables are the slot configuration (or shape), the slot gap, and the fan power. The primary variable is the fan power because it is the most readily changed. The slot gap is the next quickest to change and was therefore the secondary variable and the slot configuration is the tertiary variable as it is the most difficult to change. One change was made to the test matrix in Fig. 5-1during the testing. This change is that the smallest slot gap with the cusp installed was skipped due to tunnel time restrictions. This resulted in 54 distinct data points. 


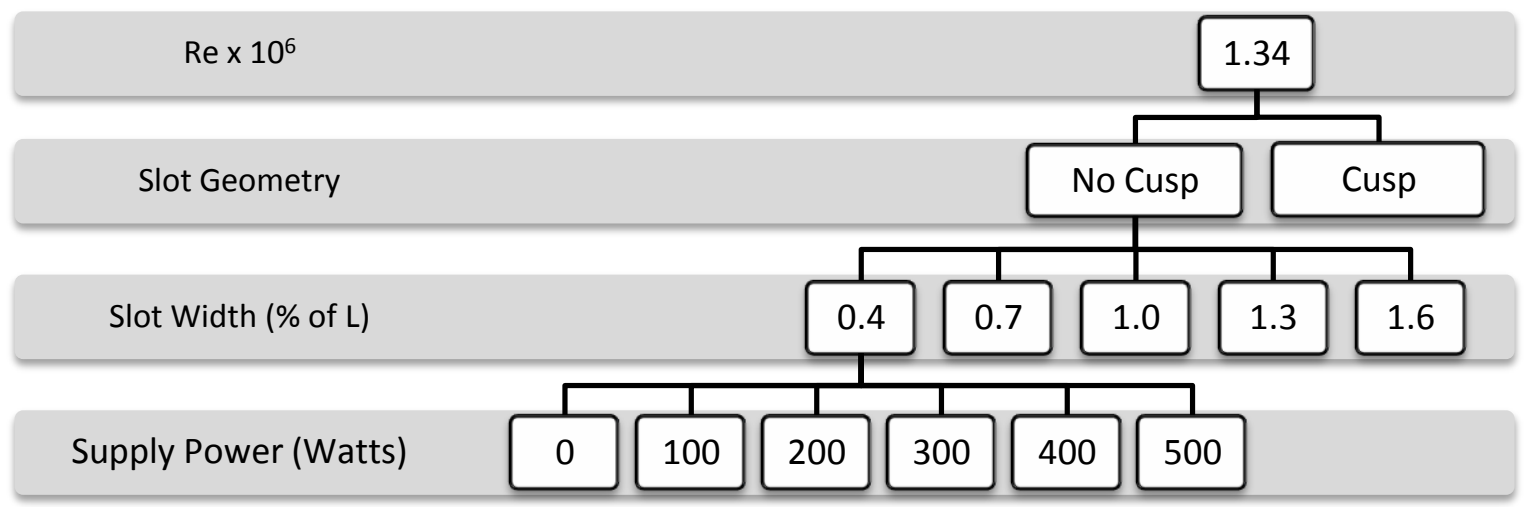

Figure 5-1. Test matrix for the wind tunnel test of the NGP. The smallest slot gap with the cusp was not run, resulting in 54 total test points.

After the half of the test matrix for the no-cusp configuration was completed, nine repeat data points were taken to evaluate the repeatability of the data. These 9 points are given in Table 5-1. While downloading the RPM data from the ESC after the first 6 of these points, the dummy strut was added and two additional points were taken at a slot gap of $1.3 \%$. Data was recorded with the dummy strut at $0 \mathrm{~W}, 500 \mathrm{~W}$ before the dummy strut was removed, and the remaining 3 data points of the 9 were recorded. Once this was completed, the cusp was installed and the test matrix for the cusp was completed.

Table 5-1. Extra data points that were taken to evaluate the repeatability of the data.

\begin{tabular}{|c|c|c|}
\hline Repeat Point \# & Slot Gap & Supply Power \\
\hline 1 & $0.7 \%$ of $\mathrm{L}$ & $400 \mathrm{~W}$ \\
\hline 2 & $1.0 \%$ of $\mathrm{L}$ & $300 \mathrm{~W}$ \\
\hline 3 & $1.0 \%$ of $\mathrm{L}$ & $500 \mathrm{~W}$ \\
\hline 4 & $1.3 \%$ of $\mathrm{L}$ & $200 \mathrm{~W}$ \\
\hline 5 & $1.3 \%$ of $\mathrm{L}$ & $400 \mathrm{~W}$ \\
\hline 6 & $1.3 \%$ of $\mathrm{L}$ & $500 \mathrm{~W}$ \\
\hline 7 & $1.6 \%$ of $\mathrm{L}$ & $100 \mathrm{~W}$ \\
\hline 8 & $1.6 \%$ of $\mathrm{L}$ & $300 \mathrm{~W}$ \\
\hline 9 & $1.6 \%$ of $\mathrm{L}$ & $500 \mathrm{~W}$ \\
\hline
\end{tabular}

\subsection{TUFT FLOW VISUALIZATION}

The flow visualization was instrumental in determining when attached flow was achieved, and judging the "quality", or steadiness, of the attachment. As previously mentioned, test data was taken at a higher tunnel speed 
prior to the tuft visualization. This original data was accompanied by smoke flow visualization and was reported by Thomason. ${ }^{20}$ Once the tuft flow visualization had occurred, it became apparent that the flow was not fully attached at any slot gap, even at full fan power, for this high tunnel speed of $98 \mathrm{ft} / \mathrm{s}(30 \mathrm{~m} / \mathrm{s})$. The tunnel speed was lowered to $82 \mathrm{ft} / \mathrm{s}(25 \mathrm{~m} / \mathrm{s})$ and the flow was re-evaluated. At this tunnel speed, the flow would almost achieve fully attached flow at the maximum fan power, but it was very unsteady. The flow would sporadically separate, with the separation lasting less than a second and the attachment lasting about 1-2 seconds. The tunnel speed was lowered again to 66 $\mathrm{ft} / \mathrm{s}(20 \mathrm{~m} / \mathrm{s})$ and the flow was re-evaluated. At this tunnel speed, the flow was attached consistently with no unsteadiness at less than full shaft power and at multiple slot gaps.

The tufts were $1 / 32$ " orange string. They were about $0.5 "-1.5$ " in length. They were help in place on the model with thin strips of blue painter's tape. They were spaced out as shown in Fig. 5-2 and Fig. 5-3. Part of this visualization, the flow aft of the fairing was evaluated qualitatively and it was determined that the flow in this area did not suffer from any severe unsteadiness from the wake of the fairing. 


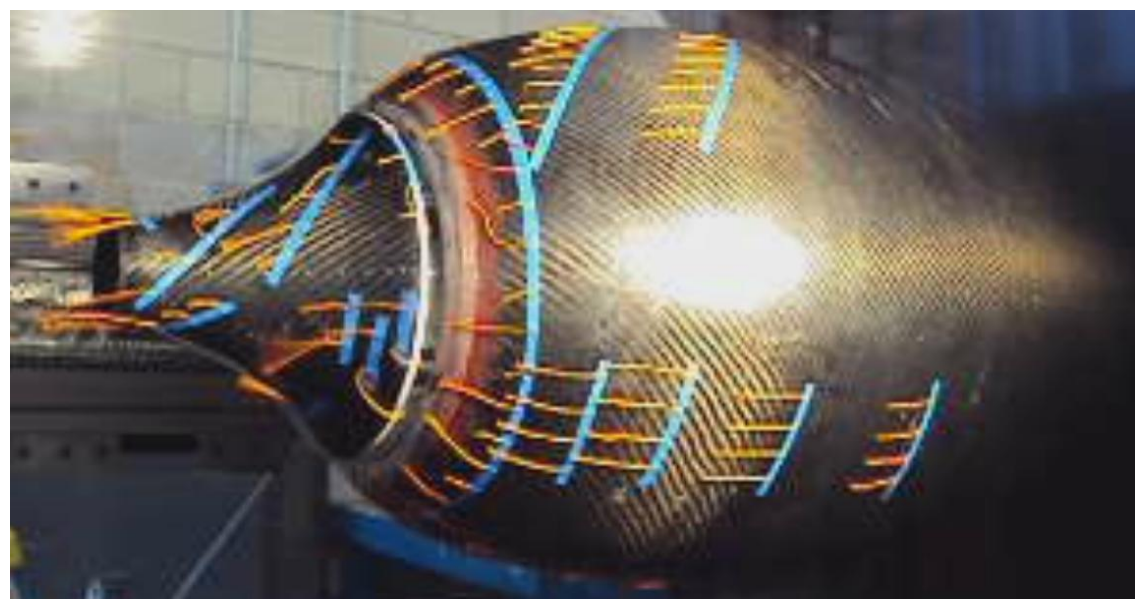

Figure 5-2. Tuft flow visualization showing fully attached flow on the aftbody

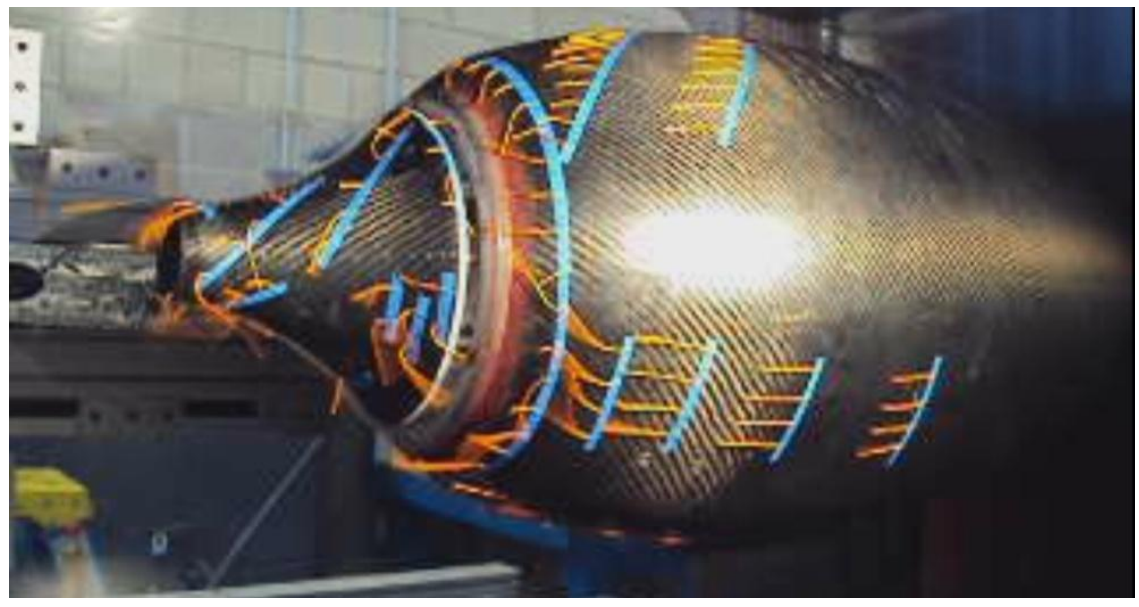

Figure 5-3. Tuft flow visualization showing fully separated flow on the aftbody 


\section{RESULTS AND DISCUSSION}

The first part of the test matrix is without a cusp at the suction slot. This data will be presented first. It will be followed by a comparison between the no-cusp and cusp data sets. This will be followed by a comparison between the no-cusp data and the nine repeated data points. This will be followed by the fan performance. Lastly, a comparison will be presented between the data of the current test and data from the 1956 Goldschmied test.

For the sake of simplicity and brevity, the following notations will be followed through the rest of this report. The abbreviation "NCx", with the " $\mathrm{x}$ " replaced by a number between 1 and 5, will represent a "No Cusp" configuration. The " $\mathrm{x}$ " represents the slot gap number. A " 1 " in this notation represents the smallest gap of $0.4 \%$ of the model length, while a "5" represents the largest gap of $1.6 \%$ of the length. These configurations are summarized in Table 6-1.

Table 6-1. Table describing the notation used for the different test parameters.

\begin{tabular}{|c|c|c|}
\hline Notation & Model Configuration & Slot gap \\
\hline NC1 & No Cusp & $0.4 \%$ of $\mathrm{L}$ \\
\hline NC2 & No Cusp & $0.7 \%$ of $\mathrm{L}$ \\
\hline NC3 & No Cusp & $1.0 \%$ of $\mathrm{L}$ \\
\hline NC4 & No Cusp & $1.3 \%$ of $\mathrm{L}$ \\
\hline NC5 & No Cusp & $1.6 \%$ of $\mathrm{L}$ \\
\hline C2 & Cusp A & $0.7 \%$ of $\mathrm{L}$ \\
\hline C3 & Cusp A & $1.0 \%$ of $\mathrm{L}$ \\
\hline C4 & Cusp A & $1.3 \%$ of $\mathrm{L}$ \\
\hline C5 & Cusp A & $1.6 \%$ of $\mathrm{L}$ \\
\hline
\end{tabular}

During the data reduction process, it was noted that data from one pressure port was very suspect. It was Scanivalve port number 58, which is the fifth port aft of the slot on the aftbody. The data recorded from the slot was always closer to a zero gauge pressure than the ports surrounding it. This trend was present when the gauge pressure was both positive and negative. This indicates that there was an undetected leak in the lines between the port and the Scanivalve. This occurred in spite of rigorously checking every line for leaks prior to testing. This port was eliminated from all data sets. 


\subsection{NO-CUSP CONFIGURATION}

The no-cusp configuration was a simple gap slot at the suction slot entrance. The most basic parameter is the surface pressure measurements. This data will serve as a foundation for the pressure drag calculations presented next. The pressure drag data is presented in two forms. The first form shows the pressure distribution for various shaft power settings and constant slot gaps, and the second is with various slot gaps and constant fan power settings. The first form is actually how the data was taken in the tunnel. The slot gap was help constant, and the power setting was varied and data taken at each point. The second form is equivalent to holding the power-setting constant but varying the slot gap.

\subsubsection{Pressure Distribution}

The pressure coefficient distributions presented in Fig. 6-1 through Fig. 6-5 are each a constant slot gap. The pressure coefficient distributions presented in Fig. 6-6 through Fig. 6-11 are each a constant fan shaft power. These figures show a lot of information about what is occurring on the surface of the model. The first thing is that the pressures over the forward part of the forbody, which is forward of maximum thickness, match up very well for every test point. This indicates that the suction slot or fan power does not affect the surface pressure distribution much farther forward then the maximum thickness.

The NC1 configuration in Fig. 6-1 shows a consistent pressure distribution for all shaft power settings except the highest fan power setting. The $427 \mathrm{~W}$ case shows a distinctly different trend on the aftbody. This difference divides the data points into two groups. The lower power cases are at least partially separated cases, while the high power case is likely to have fully attached flow on the aftbody. The high power case has a positive pressure coefficient on the entire aftbody. It also has a characteristic step, or jump, in the pressure distribution at the slot, while all other lower power settings are somewhat continuous with the pressure distribution forward of the suction slot. Another distinct characteristic of the highest power case is the less adverse pressure gradient aft of the maximum thickness, followed by a small region forward of the suction slot with a much larger adverse pressure gradient. This is in contrast to the lower power cases where the pressure gradient turns adverse almost immediately after the maximum thickness and continues until the trailing edge of the model.

When comparing the multiple figures, a general trend emerges. The power required to achieve attached flow is reduced as the slot gap gets larger. For the NC2 configuration, attached flow likely occurs at a slightly higher 
power than $258 \mathrm{~W}$. for the NC3 configuration, the power required for attached flow shifts to between $171 \mathrm{~W}$ and $258 \mathrm{~W}$. The NC4 configuration requires about $171 \mathrm{~W}$ to achieve attached flow, and the NC5 configuration achieves fully attached flow for all data points with a non-zero fan power setting.

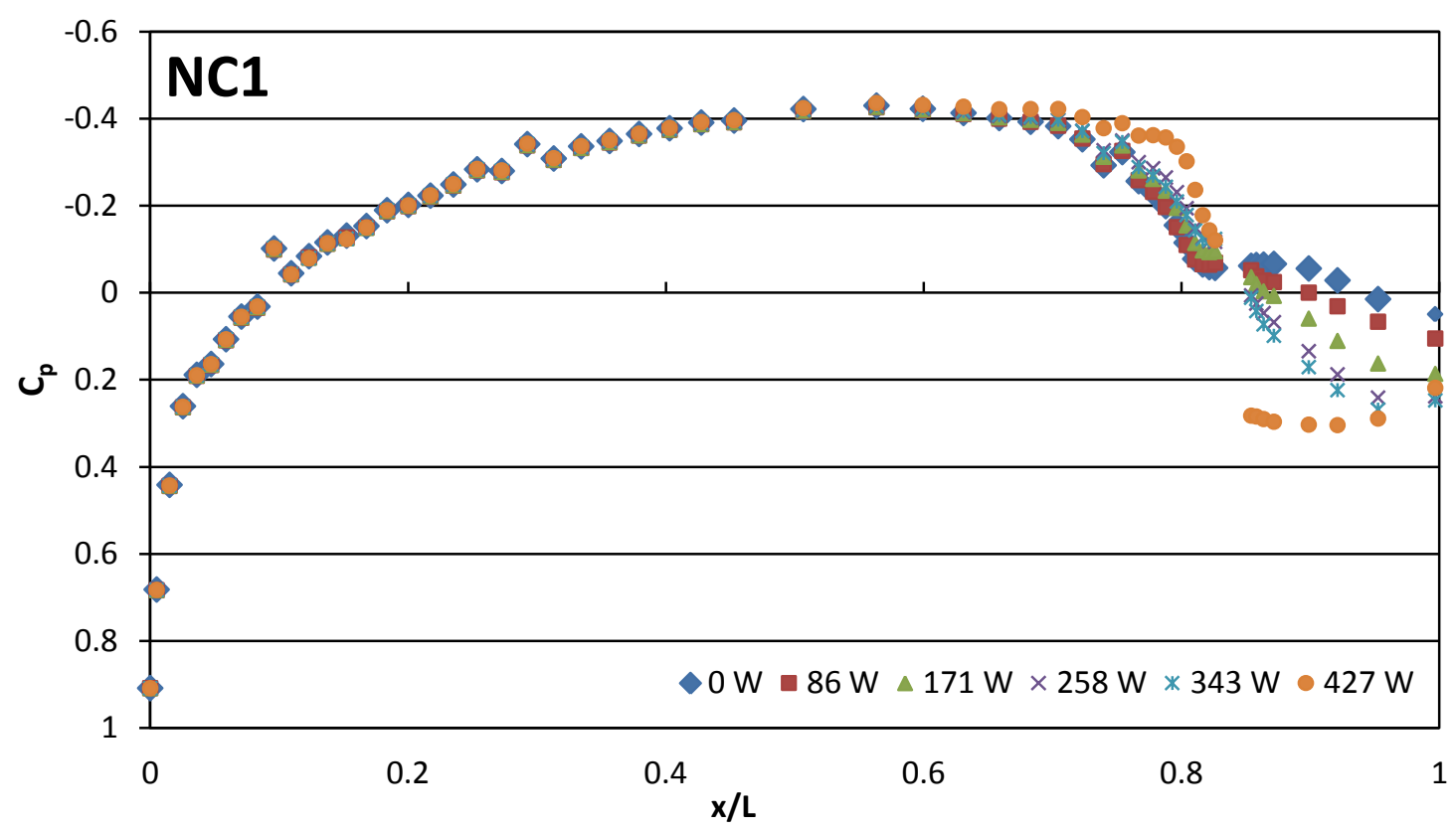

Figure 6-1. $C_{p}$ distribution for the case with no cusp and a slot gap of $0.4 \%$ of the body length. 


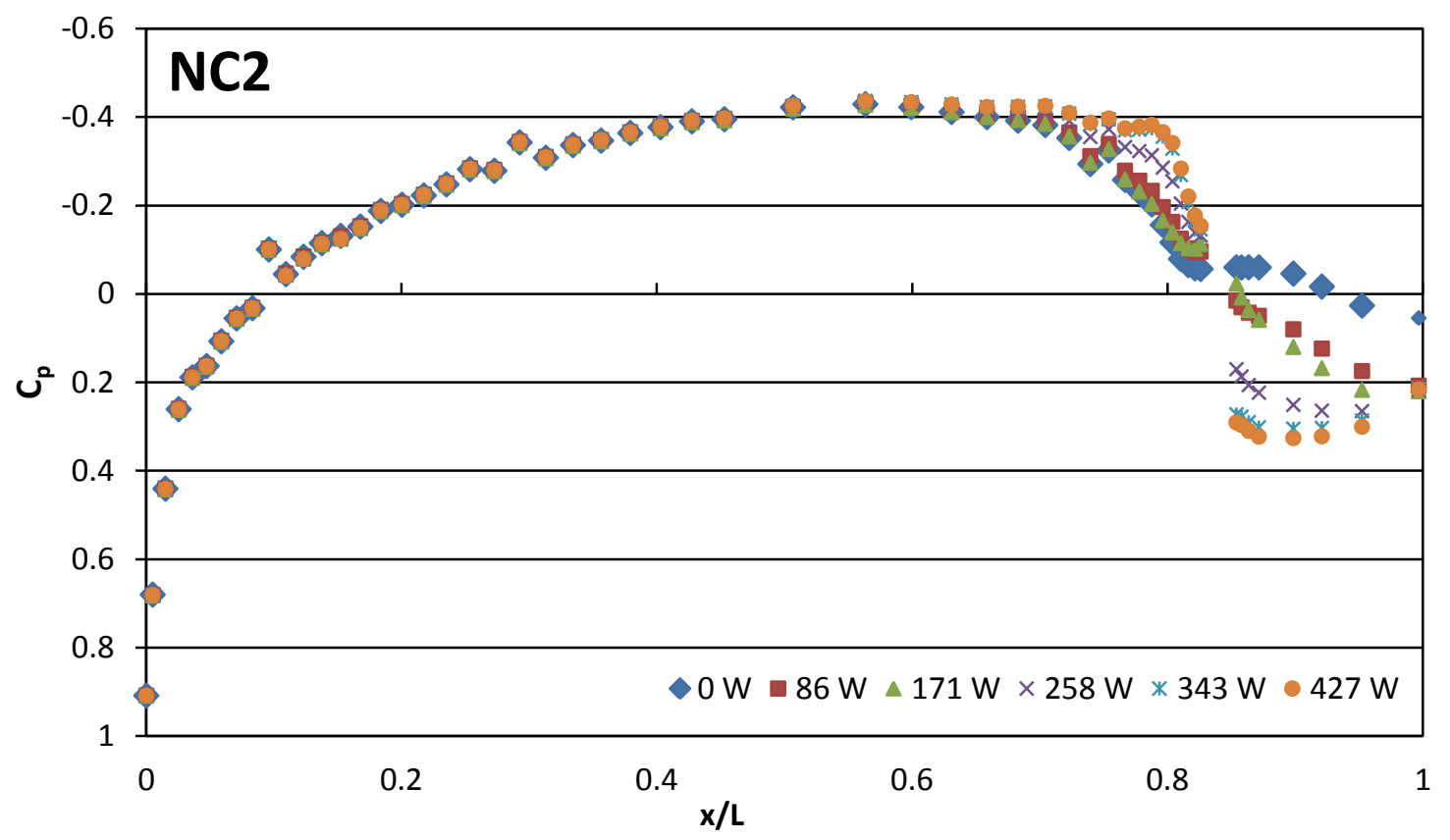

Figure 6-2. $C_{p}$ distribution for the case with no cusp and a slot gap of $0.7 \%$ of the body length.

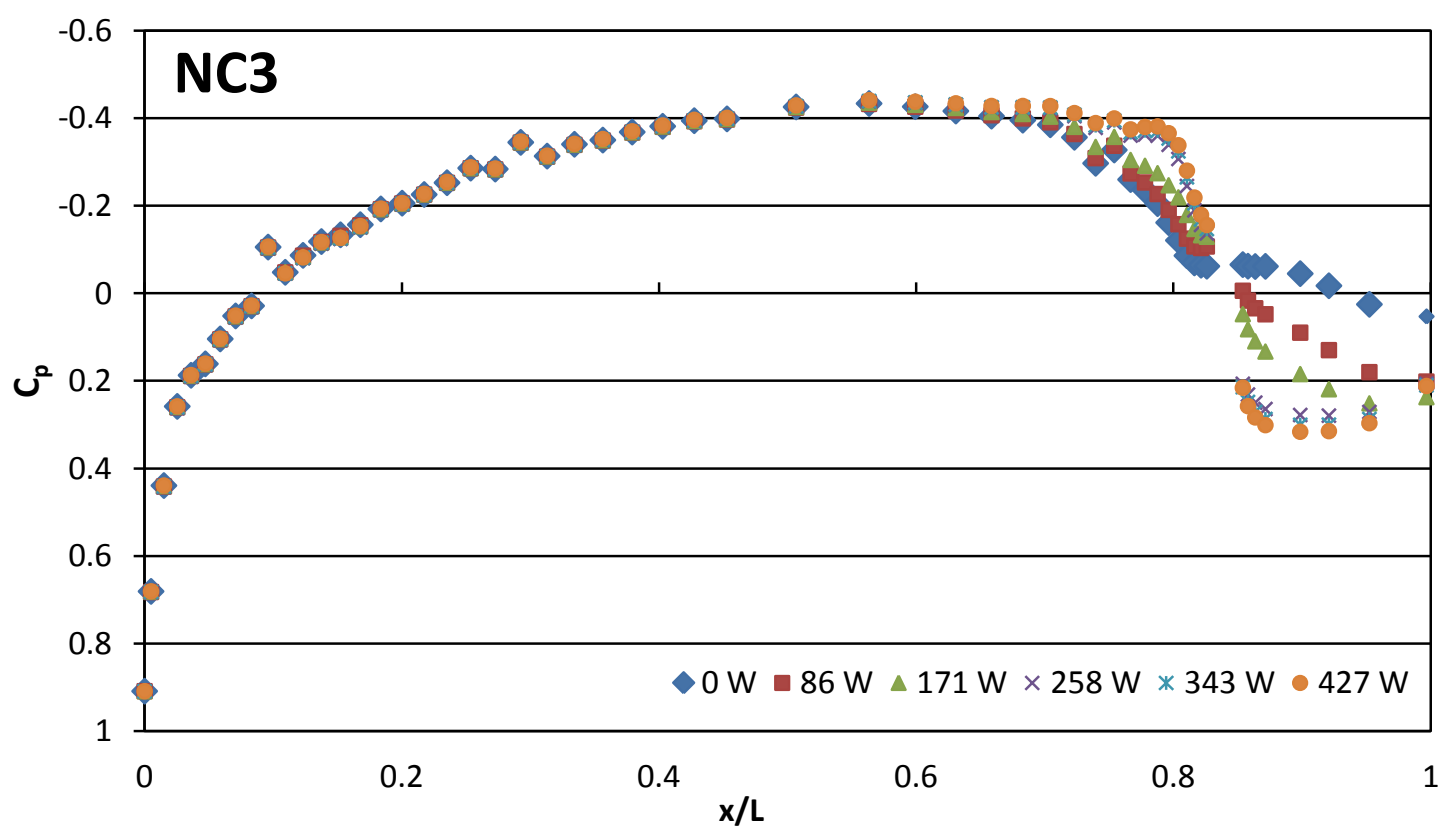

Figure 6-3. $C_{p}$ distribution for the case with no cusp and a slot gap of $1.0 \%$ of the body length. 


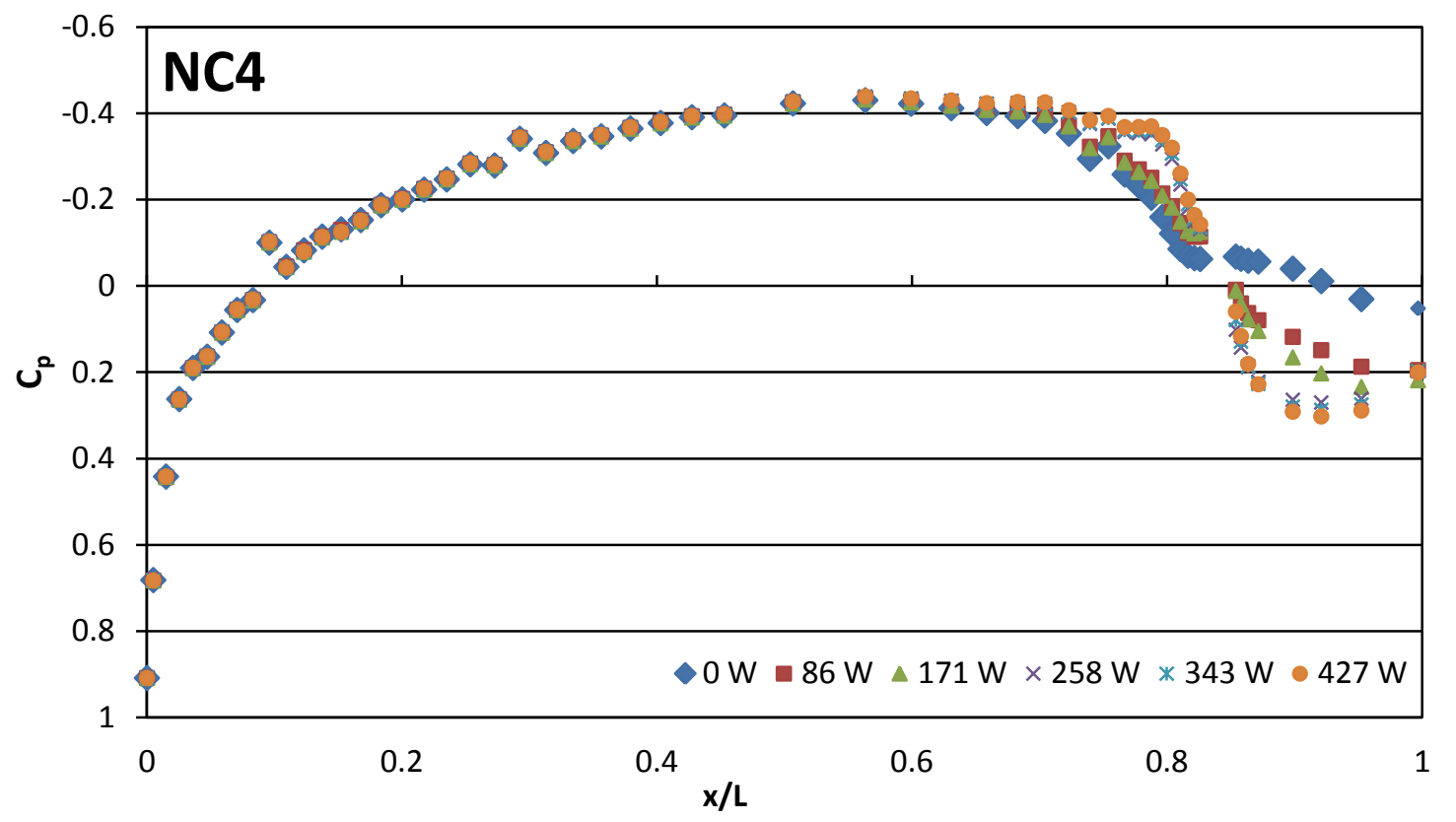

Figure 6-4. $C_{p}$ distribution for the case with no cusp and a slot gap of $1.3 \%$ of the body length.

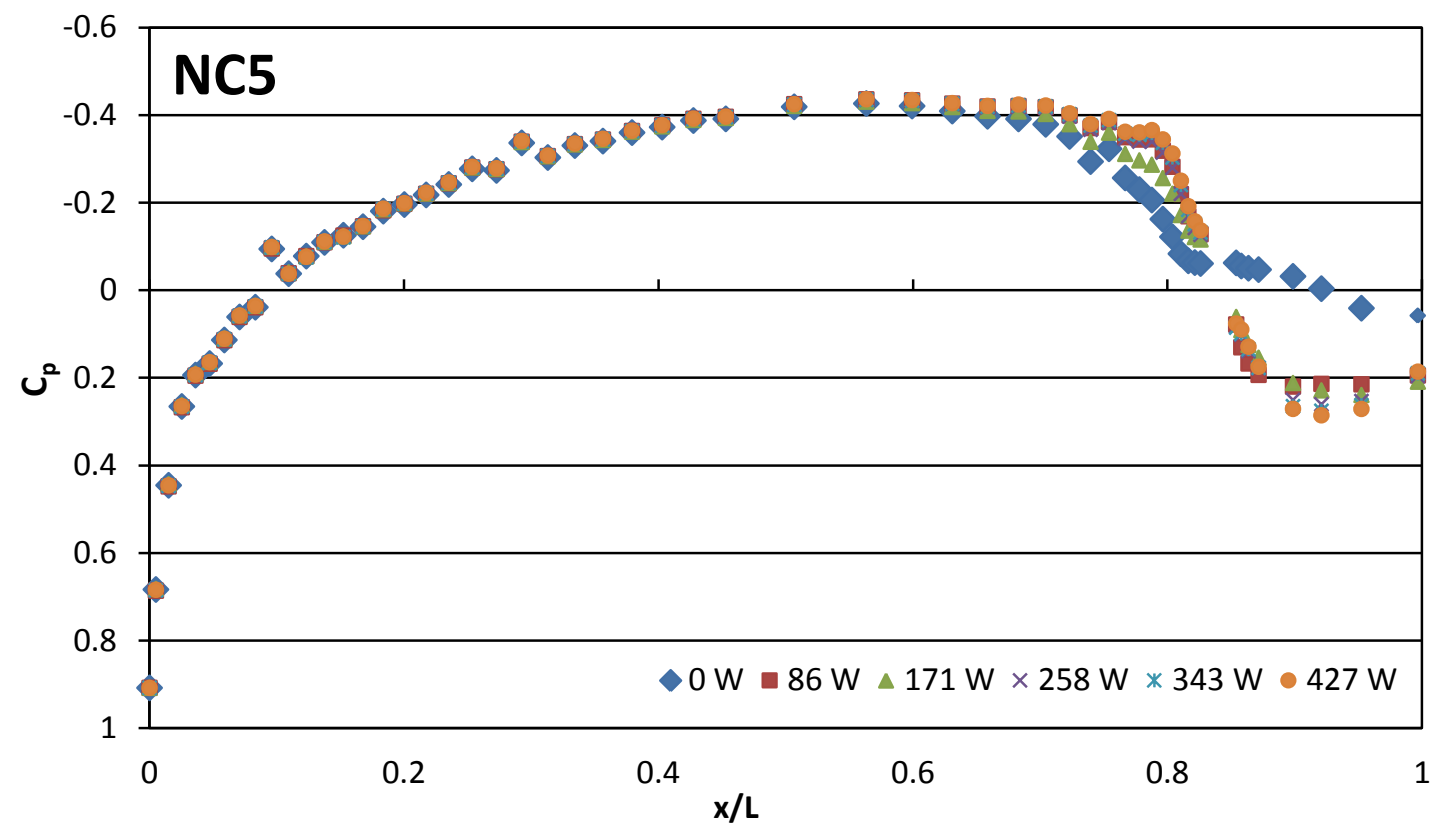

Figure 6-5. $C_{p}$ distribution for the case with no cusp and a slot gap of $1.6 \%$ of the body length.

The same data set as presented in Fig. 6-1 through Fig. 6-5 will now be presented in a slightly altered form. Fig. 6-6 through Fig. 6-11 is for a constant fan power setting and shows the variation in the $\mathrm{C}_{\mathrm{p}}$ distribution for the 
various slot gaps. These figures also show some definite trends in the data. The first trend is that the data points with the model fan off demonstrate that the pressure distribution is very repeatable and constant for all slot gaps. The only variation is on the aftbody, and it is very slight. This repeatability is in spite of the considerably unsteady separated wake that this pressure distribution was measured from. The separation point is assumed just upstream of the suction slot because this is where the pressure gradient becomes zero, as is characteristic of a separation point.

For the data points with the fan running, similar trends can be seen. Each constant power group of data points can be divided into the same groups as previously described, those data points that are attached, and those that are fully or partially separated.

For the case of $86 \mathrm{~W}$ shown in Fig. 6-7, the only data point that is attached is the largest slot gap of 1.6\%. It shows the characteristic less adverse pressure gradient after the maximum thickness followed by a more severe adverse pressure gradient just upstream and downstream of the slot. The next case of $171 \mathrm{~W}$ shows almost evenly spaced distributions between a fully separated case and the fully attached case. The largest two slot gaps are likely attached, and the smallest two slot gaps are likely separated, with the middle slot gap being partially separated. Exact quantification of the amount of separation and the classification of whether or not the flow over the aftbody is separated or attached is not straightforward for this group of data points. The pressure gradient is more adverse aft of the maximum thickness than the fully attached case from Fig. 6-6, and it is less adverse just upstream and downstream of the slot. The next two higher fan power settings of $258 \mathrm{~W}$ in Fig. 6-9 and $343 \mathrm{~W}$ in Fig. 6-10 are clearer. They both show that all the data points are attached except for the smallest slot gap. The highest fan power of $427 \mathrm{~W}$ in Fig. 6-11 simply shows that for all slot gap settings, the flow is attached on the aftbody. One interesting feature of this fan power setting is that the entire pressure distribution between the leading tip of the model and the suction slot is very similar for all cases. The only difference is the pressure distribution aft of the slot.

The last interesting trend is that for some fan power setting, the data points with a larger slot gap actually had a lower surface pressure just aft of the slot, but it quickly reaches the same value of $\mathrm{C}_{\mathrm{p}}$ as the smaller slot data points. This can be seen clearly in Fig. 6-10. As the slot gap became larger, the surface pressure aft of the slot decreased in spite of the slot area enlarging and the slot velocity decreasing. 


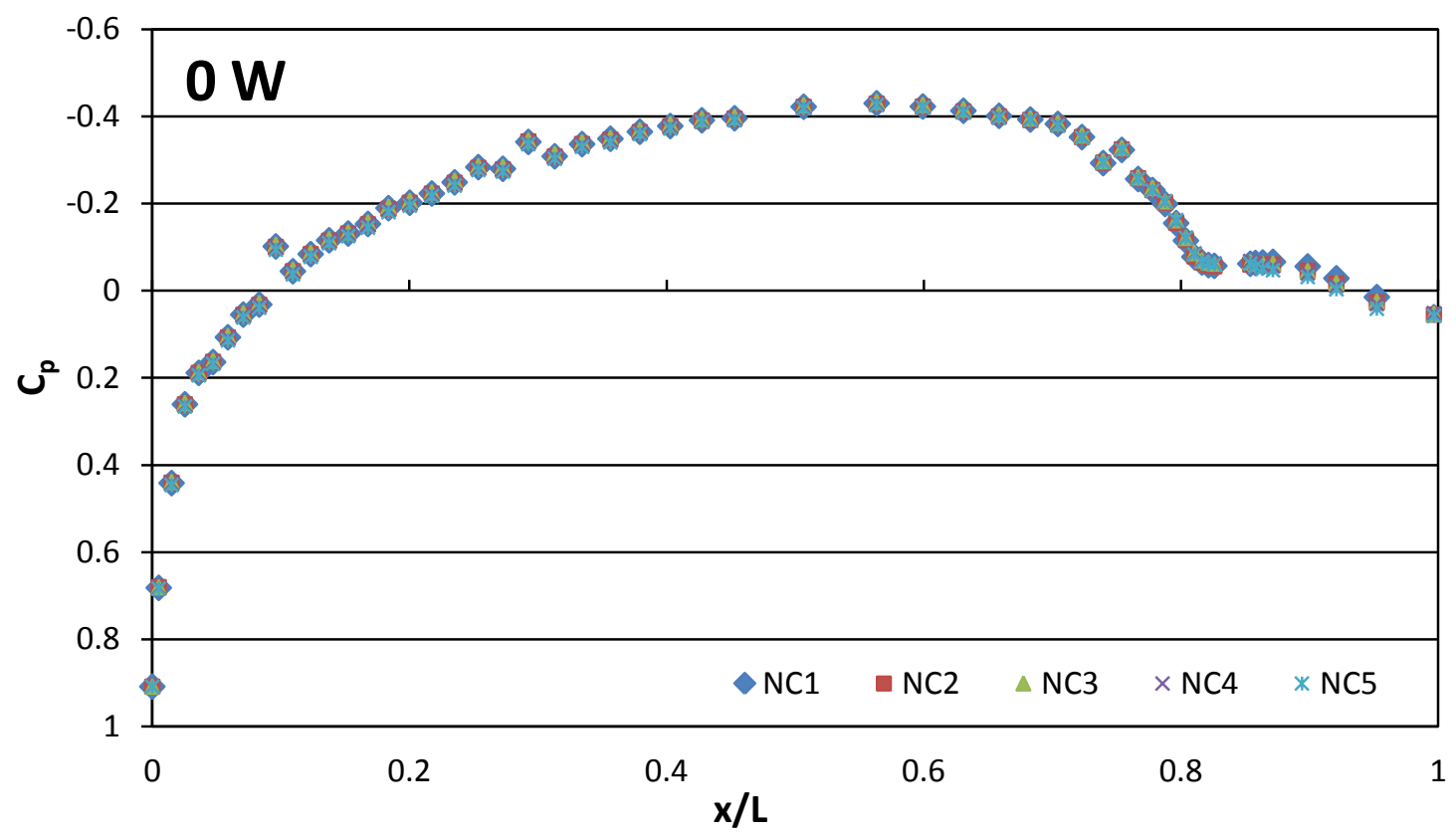

Figure 6-6. $C_{p}$ distribution for the case with no cusp and a shaft power of $0 \mathrm{~W}$ and various slot gaps.

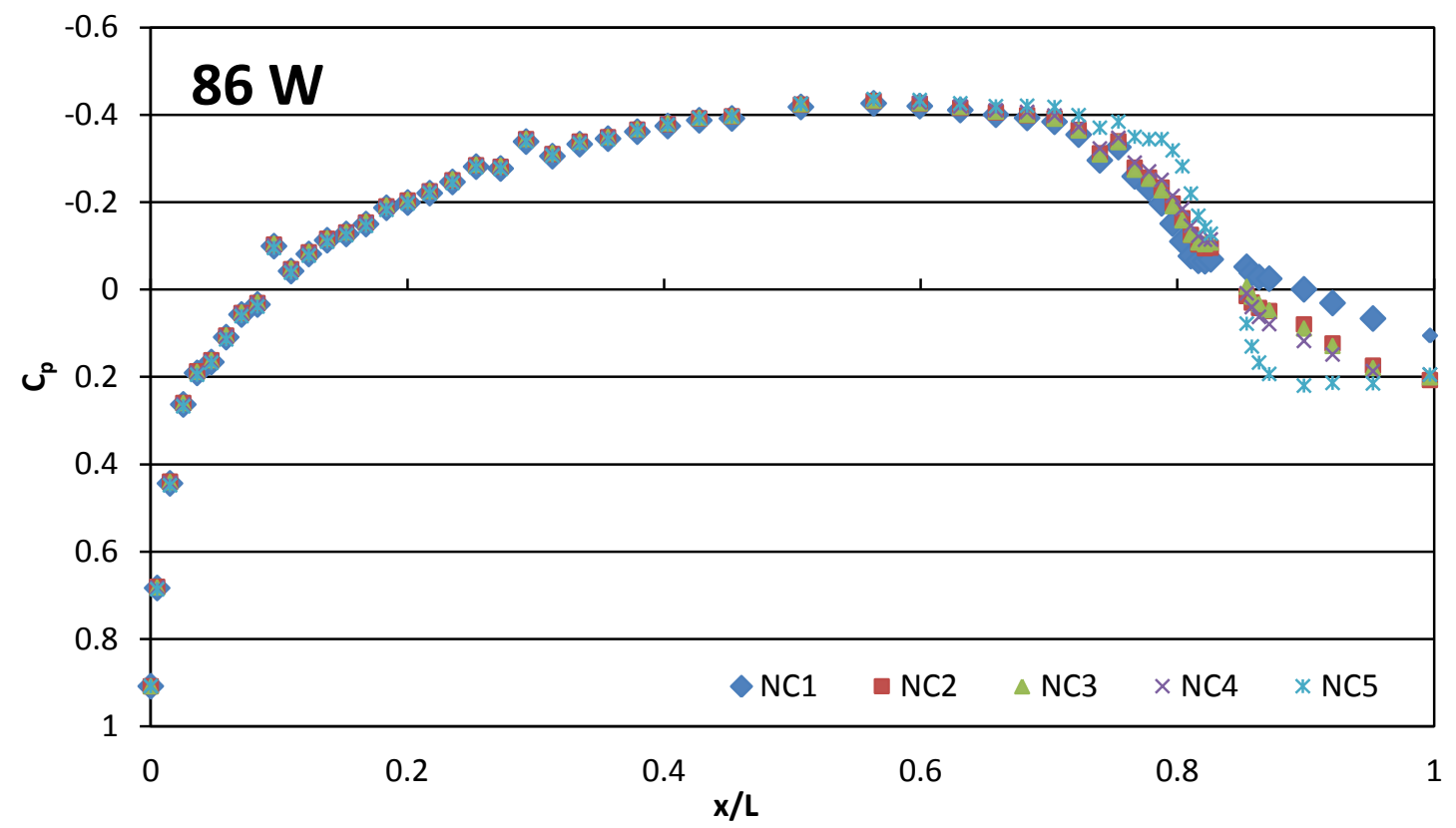

Figure 6-7. $C_{p}$ distribution for the case with no cusp and a shaft power of $86 \mathrm{~W}$ and various slot gaps. 


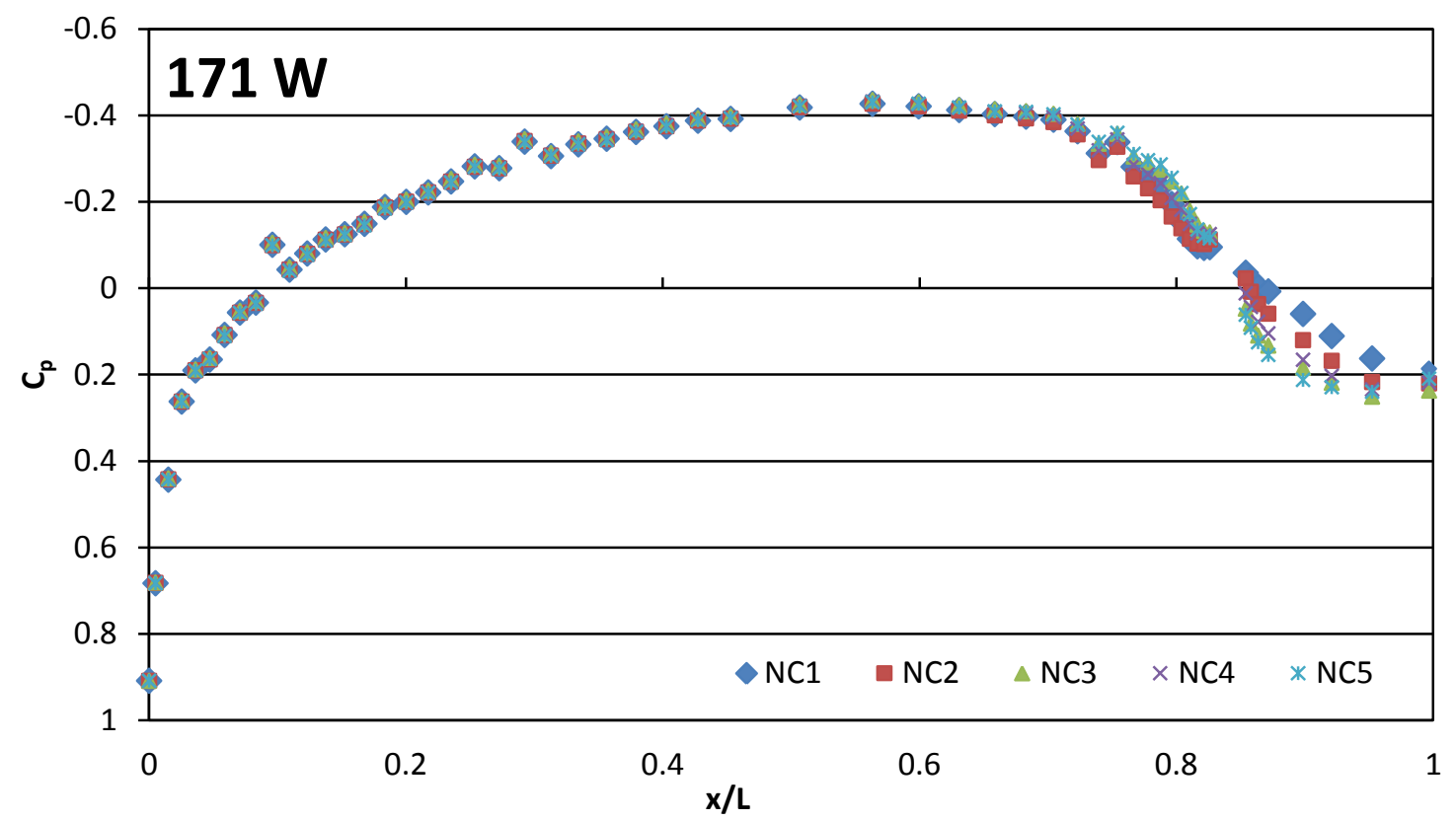

Figure 6-8. $C_{p}$ distribution for the case with no cusp and a shaft power of $171 \mathrm{~W}$ and various slot gaps.

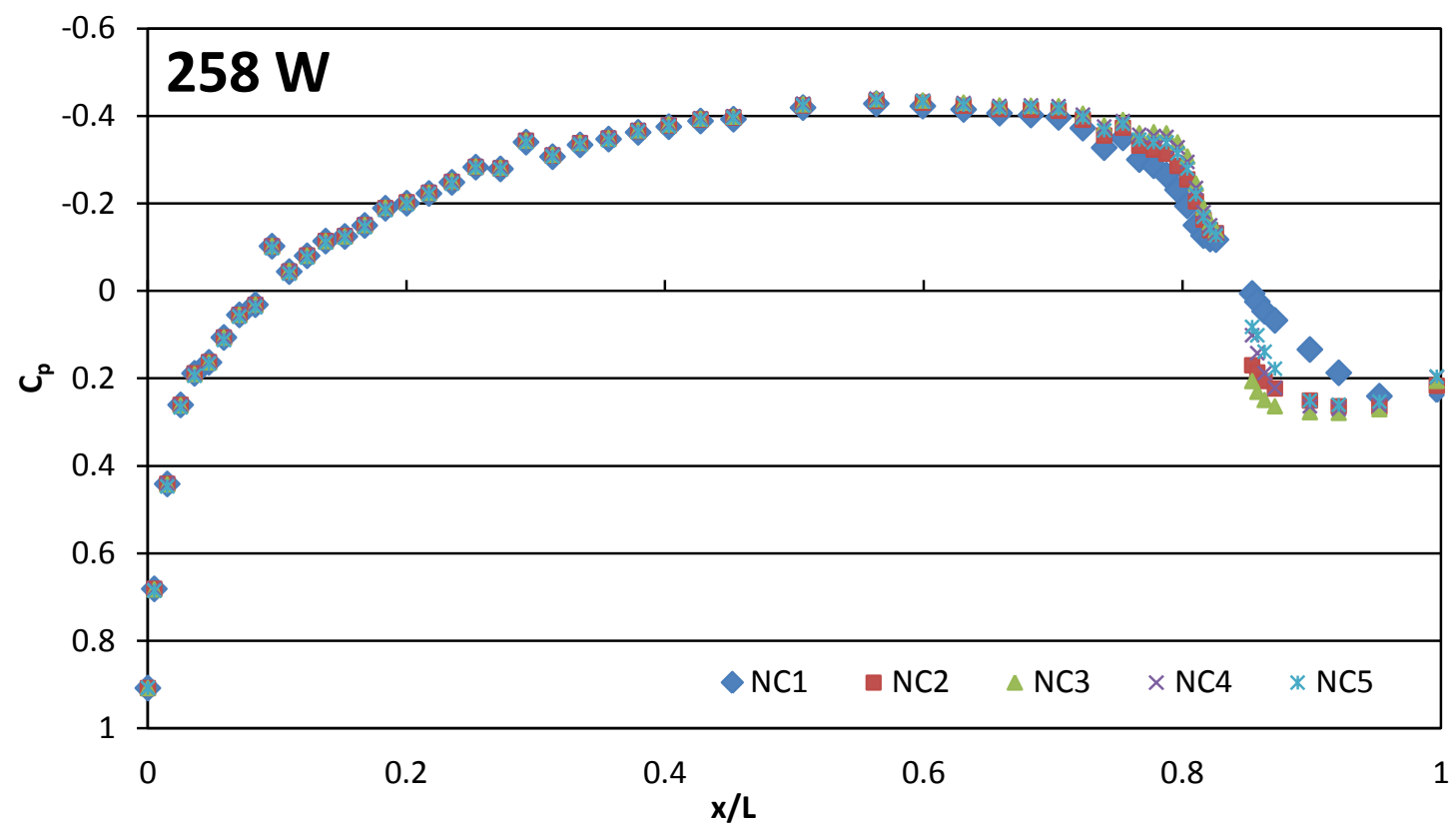

Figure 6-9. $C_{p}$ distribution for the case with no cusp and a shaft power of $258 \mathrm{~W}$ and various slot gaps. 


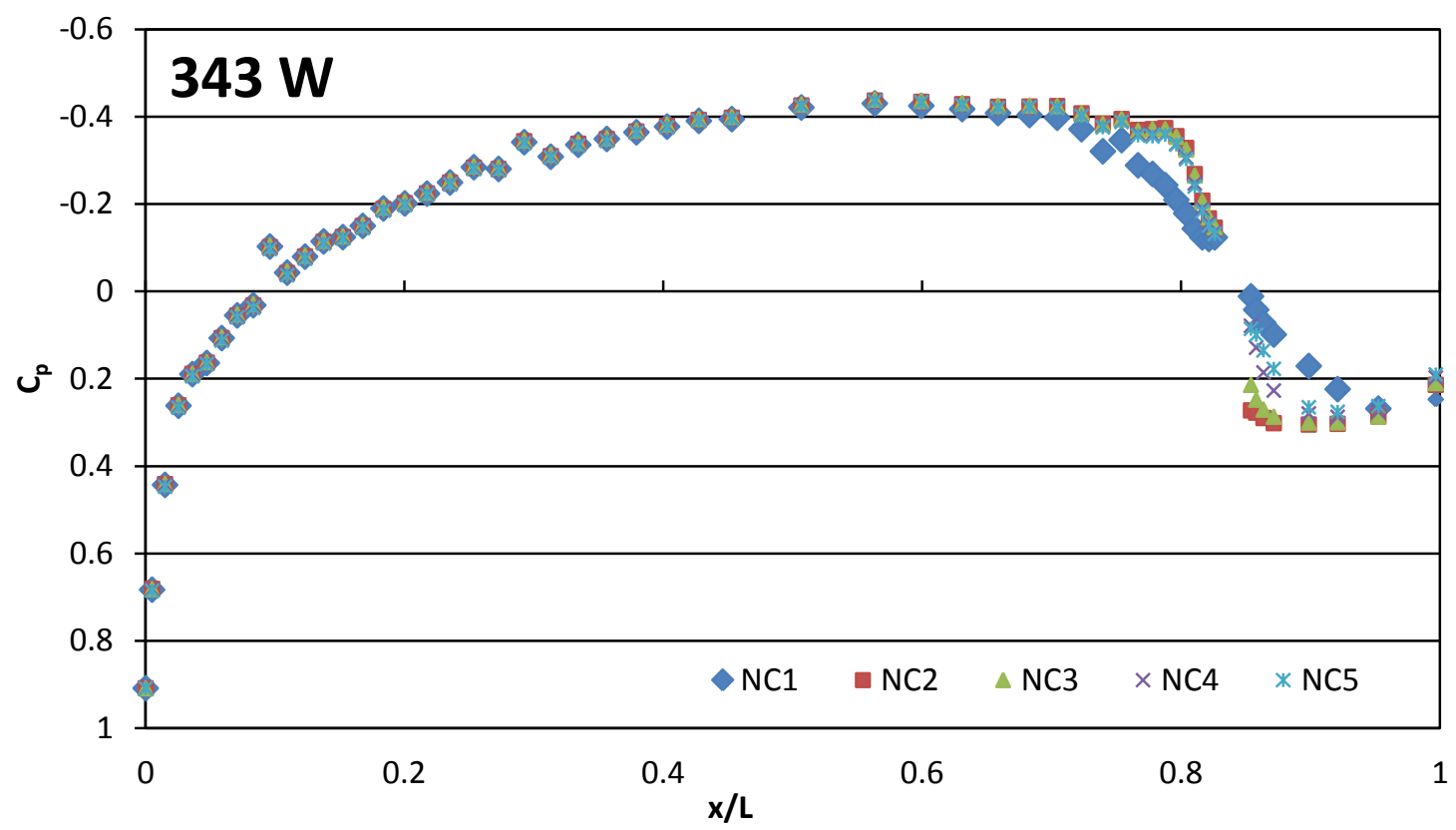

Figure 6-10. $C_{p}$ distribution for the case with no cusp and a shaft power of $343 \mathrm{~W}$ and various slot gaps.

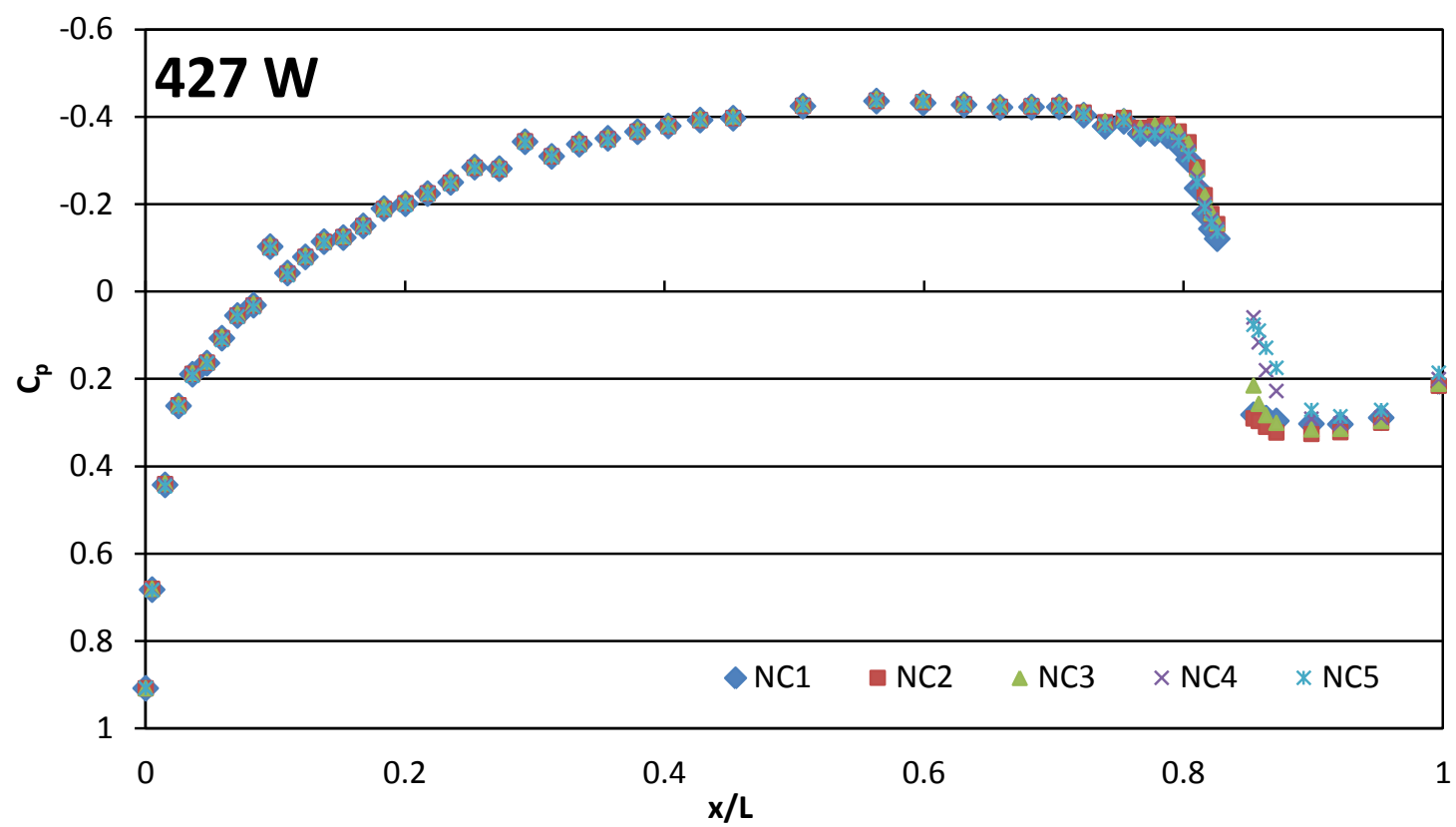

Figure 6-11. $C_{p}$ distribution for the case with no cusp and a shaft power of $427 \mathrm{~W}$ and various slot gaps. 


\subsubsection{Axial Pressure Force}

The axial pressure force coefficient, $\mathrm{C}_{\mathrm{Ap}}$, is calculated from the preceding pressure distributions. The trends in the axial pressure force data set are less clear. The first noticeable trend is that positive net pressure force is achieved for some data points. The second trend is that for most lines of constant slot gap, as the shaft power increases the pressure force get more positive, reaches a peak, and then decreases. This downward turn in the axial pressure force data is believed to be caused by too much suction. It is theorized that the suction initially causes an increase in the axial pressure force because the suction allows better pressure recovery on the aftbody from the elimination, or reduction, of the separation. However, as the suction increased further, the low pressure in the slot was able to propagate both upstream and downstream of the slot. This increased amount of low pressure on the aft side of the body caused this decrease in the axial pressure force.

For the smallest suction slot, the axial pressure force increases with increased fan power. The peak axial pressure force is at the highest suction flow rate and fan power. The NC2 slot of $0.7 \%$ of the body length reaches its peak axial pressure force at a shaft power of $171 \mathrm{~W}$. In fact, all slot gaps besides the smallest slot gap reach their peak at this same power. This indicates that for maximum positive axial pressure force, the fan needs to operate near this power setting.

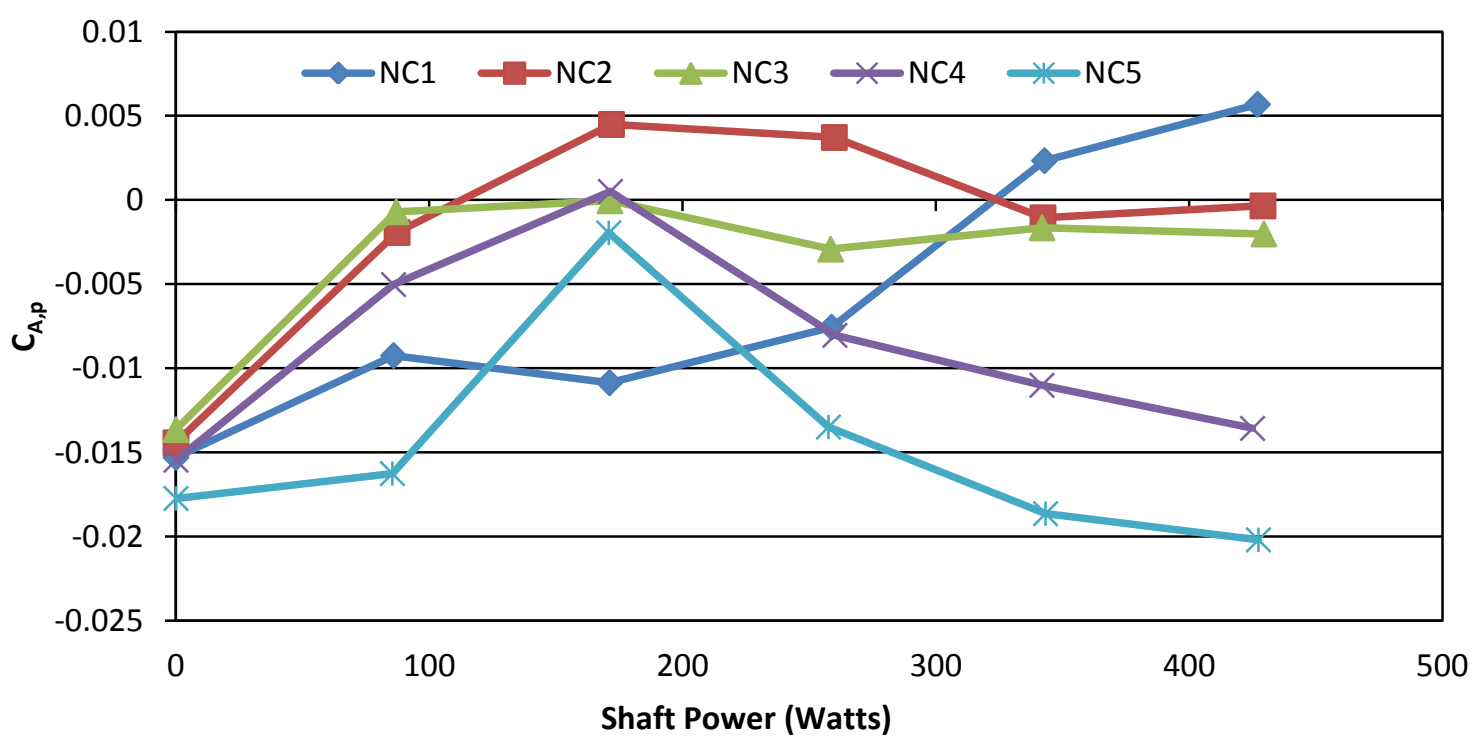

Figure 6-12. Axial pressure force coefficient for various slot gaps and fan shaft power settings. 
If instead of looking at the data in terms of constant slot gap, like Fig. 6-12, it is looked at in terms of constant power and varying slot gap, an important trend emerges. For each power setting, there is a distinct slot gap and mass flow rate that produces the highest axial pressure force. In this figure, each line represents a varying of the slot gap only. The lower suction flow rate is achieved at the smaller slot gap, and by increasing the slot gap, the mass flow increases.

For shaft power settings below $300 \mathrm{~W}$, the increased slot results in increased axial pressure force until its peaks and then any further increase in slot gap, which further increases the mass flow, causes a decrease in the axial pressure force. For shaft power settings above $300 \mathrm{~W}$, the peak axial pressure force is achieved at the lowest fan power setting and mass flow rate, and the axial pressure force decreases for all increases in slot gap and mass flow rate. The major trend that this plot demonstrates is that the axial pressure force increases as fan shaft power is increased from zero power up to about $171 \mathrm{~W}$, then it never appreciably increases with increased power. In fact, increases power can also cause a distinct decrease in the axial pressure force.

A secondary trend that is important is that by varying the slot gap, the suction flow rate is only mildly affected, especially at low shaft power settings. A larger influence on the suction flow rate is the shaft power setting. A fourth important thing that can be learned from this figure is that suction flow rate alone does not determine the axial pressure force. This is because, at the higher shaft power settings, the same flow rate can be achieved at two shaft power settings, but the lower shaft power setting will have a low axial pressure force, while the higher shaft power setting will have a higher axial pressure force. 


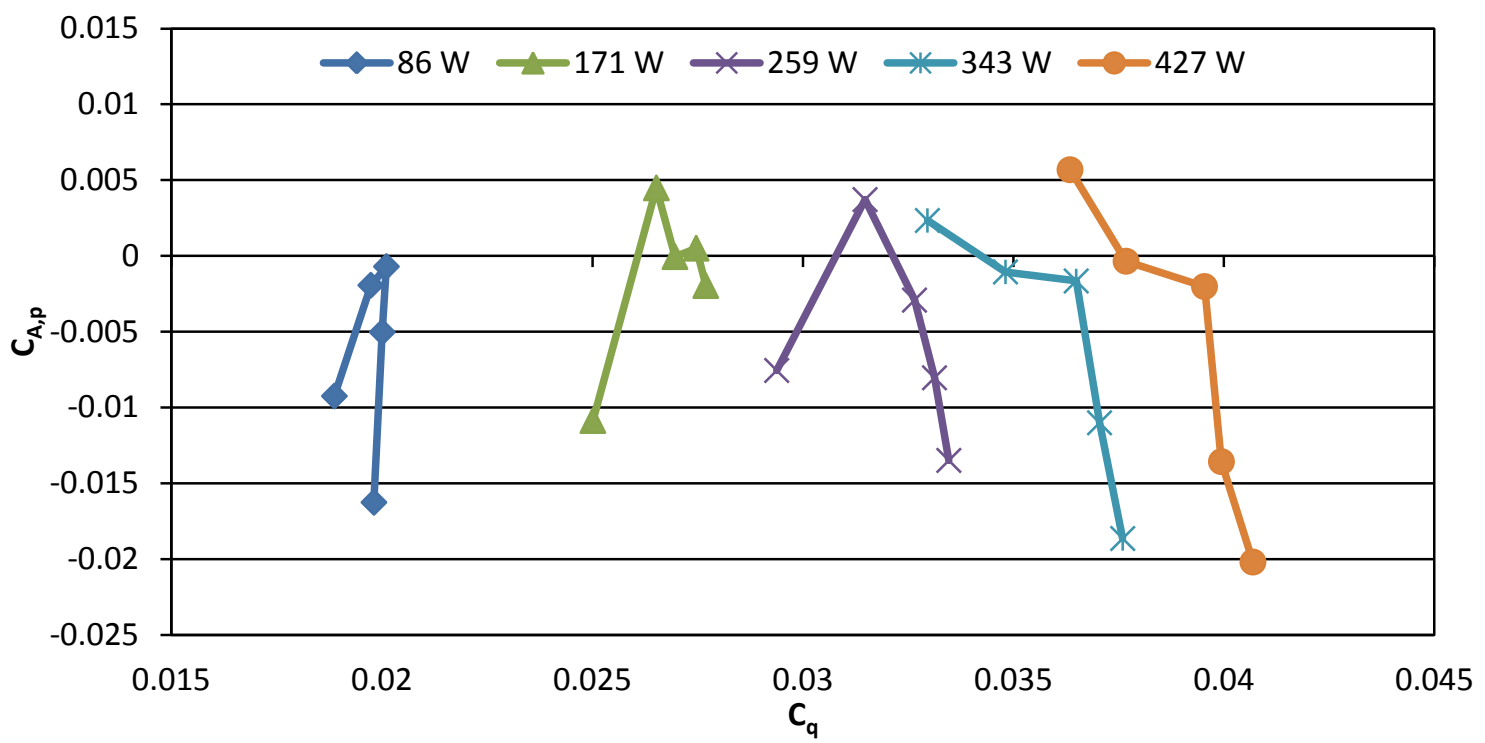

Figure 6-13. Axial pressure force as a function of mass flow rate for various power settings.

\subsubsection{TOTAL AXIAL FORCE}

The axial force coefficient, $\mathrm{C}_{\mathrm{A}}$, as a function of the fan shaft power is displayed in Fig. 6-14. The first noticeable feature of this plot is that a slot gap of $1.3 \%$ has lower axial force than the other slot gaps at all power settings. As will be discussed later, it is believed that this data point does not actually reflect the true behavior at this slot gap.

The next trend is to notice at what slot gap where the peak axial force occurs. For the $0 \mathrm{~W}$ and $86 \mathrm{~W}$ cases, the smallest slot gap results in the highest axial pressure force. For all the other cases, the slot gap with the highest axial pressure force achieved at the largest slot gap, but the axial force at $0.7 \%$ of the body length has an axial force that is about $85 \%$ of the maximum value.

The third trend is that for every slot gap, the axial force increases with increasing shaft power. This trend is unlike what is shown for the axial pressure force where the axial pressure force stayed the same or decreased after 200W. This trend is shown in Fig. 6-15. After about 200W, the axial pressure force increases almost linearly with increased shaft power, and after about $150 \mathrm{~W}-200 \mathrm{~W}$, the axial force is positive, indicating a net forward force. 


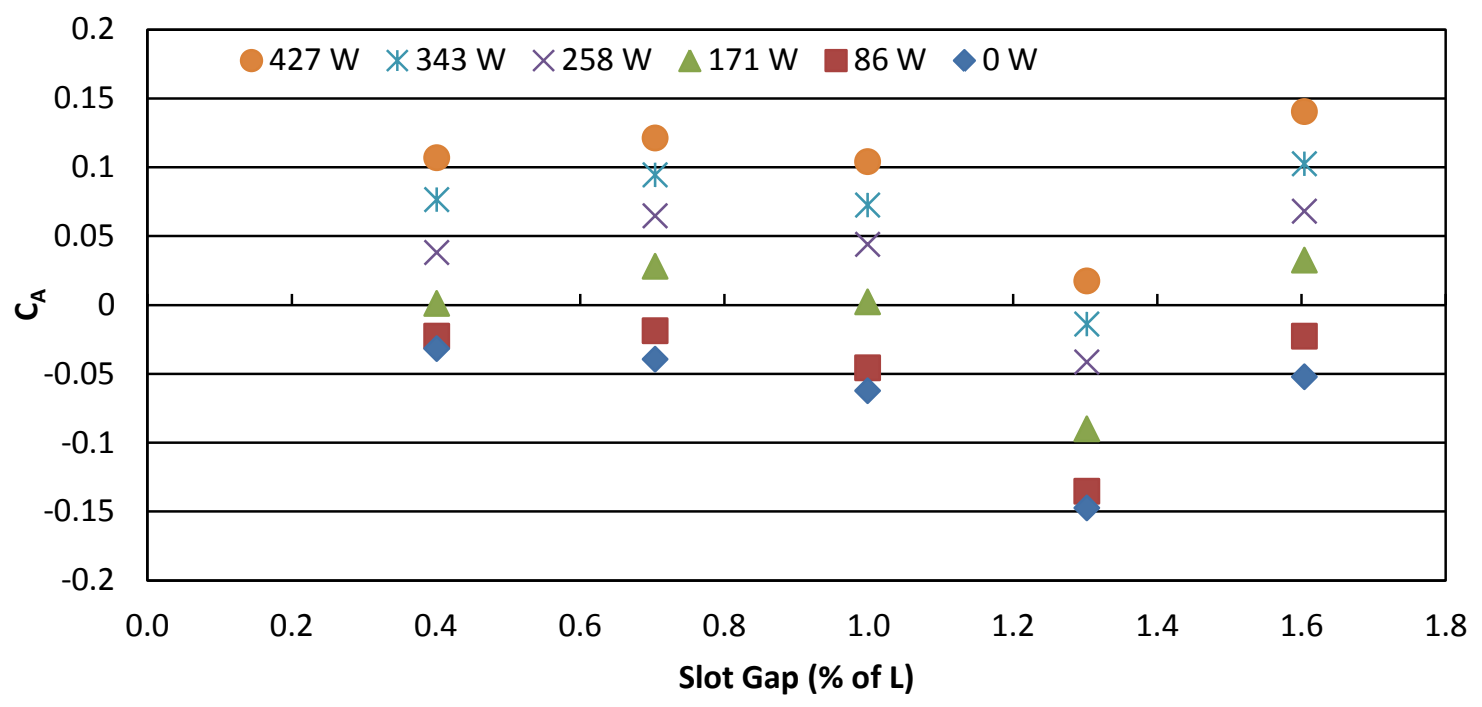

Figure 6-14. Axial force coefficient of the no-cusp cases as a function of the slot gap for various fan power settings.

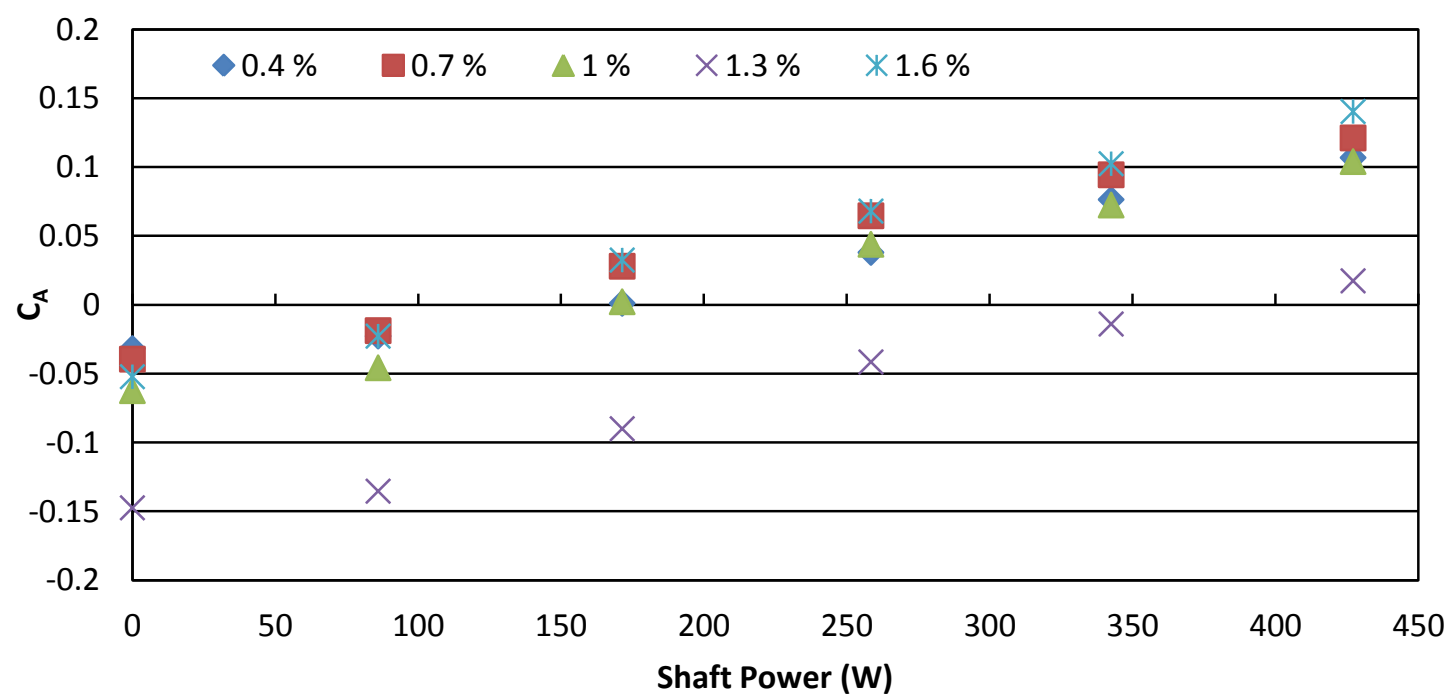

Figure 6-15. Axial force coefficient of the no-cusp cases as a function of the shaft power for various slot gaps.

The same trend is evident in Fig. 6-16 as was evident in Fig. 6-15 but with the suction flow rate instead of shaft power. As the suction flow rate increases from zero, the axial force does not change appreciably until a threshold value is reached, and then the axial force increases almost linearly with increased suction flow rate. Not 
coincidentally, this threshold value of about $0.025-0.030$ corresponds to the 171 shaft power cases. This corresponds with the trend shown in Fig. 6-15.

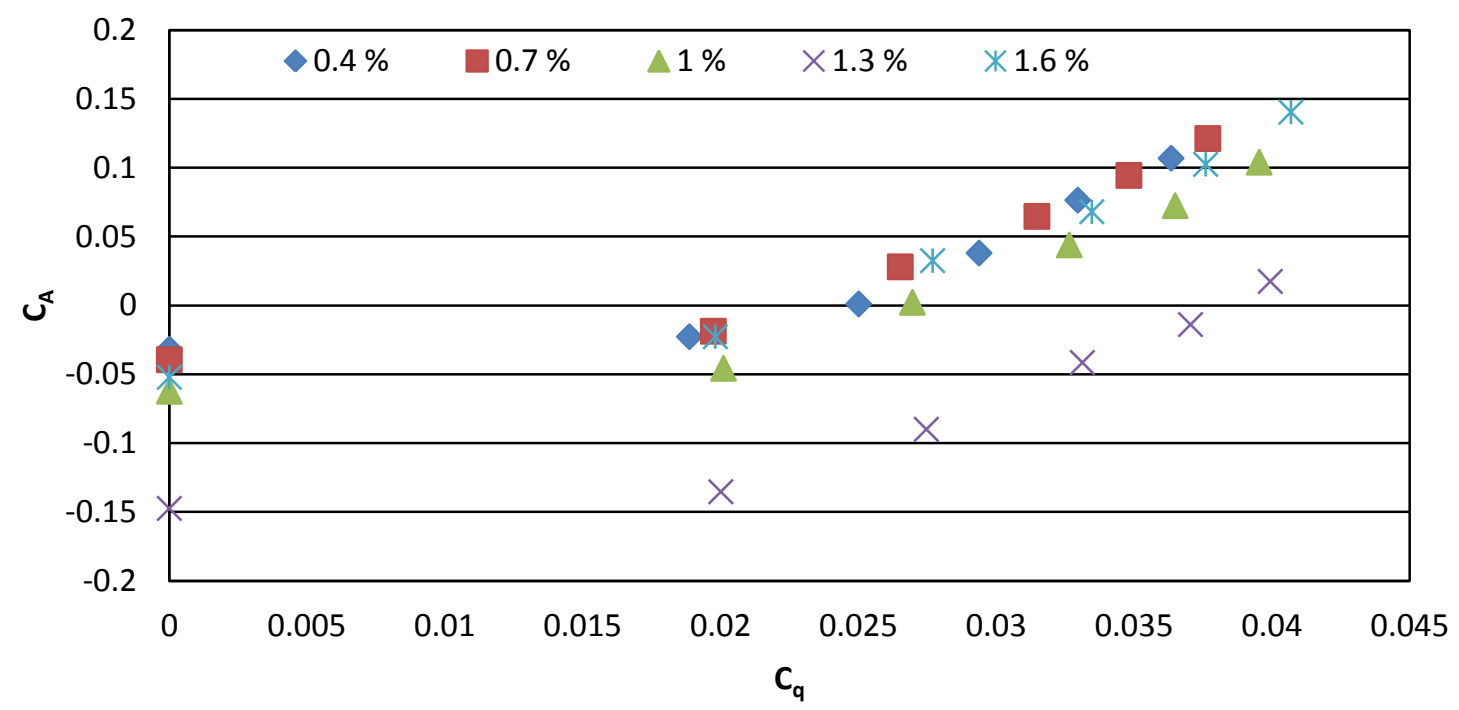

Figure 6-16. Axial force coefficient of the no-cusp cases as a function of the suction flow coefficient for various slot gaps.

\subsubsection{Slot Parameters}

The pressure rise across the slot is a parameter that was used in the previous tests to evaluate the match between the mass flow requirement for the propulsor and the mass flow requirement for the BLC. It was considered that if full aftbody attachment is achieved prior to the net axial force on the model being zero, then the propulsion system and the BLC system was viewed as well matched. For the current testing, the full aftbody attachment occurred, based on tuft flow visualization and the pressure distributions, at around the $343 \mathrm{~W}$ case for most of the slot gaps, where the net zero axial force was achieved at about the $171 \mathrm{~W}$ case. This indicates a mismatch between the propulsion system and the BLC system. This same mismatch was found to occur in the 1982 test for the basic configuration (without the tail boom), but for the configuration with the tail boom, and accompanied reduced exit area, the mismatch was eliminated. The reduced exit area changed the fan performance such that the zero axial force point occurred after full aftbody attachment had occurred. The reduction in the aftbody exit area would normally increase the exit speed of the suction flow and result in more thrust from the propulsor for the same flow rate, but in this case, it caused a delay in the net zero axial force point to a higher suction flow rate. 
The pressure step is defined as the model surface pressure on the aft side of the slot minus the model surface pressure on the forward slide of the slot. Therefore, a positive pressure rise corresponds to higher pressure on the aftbody.

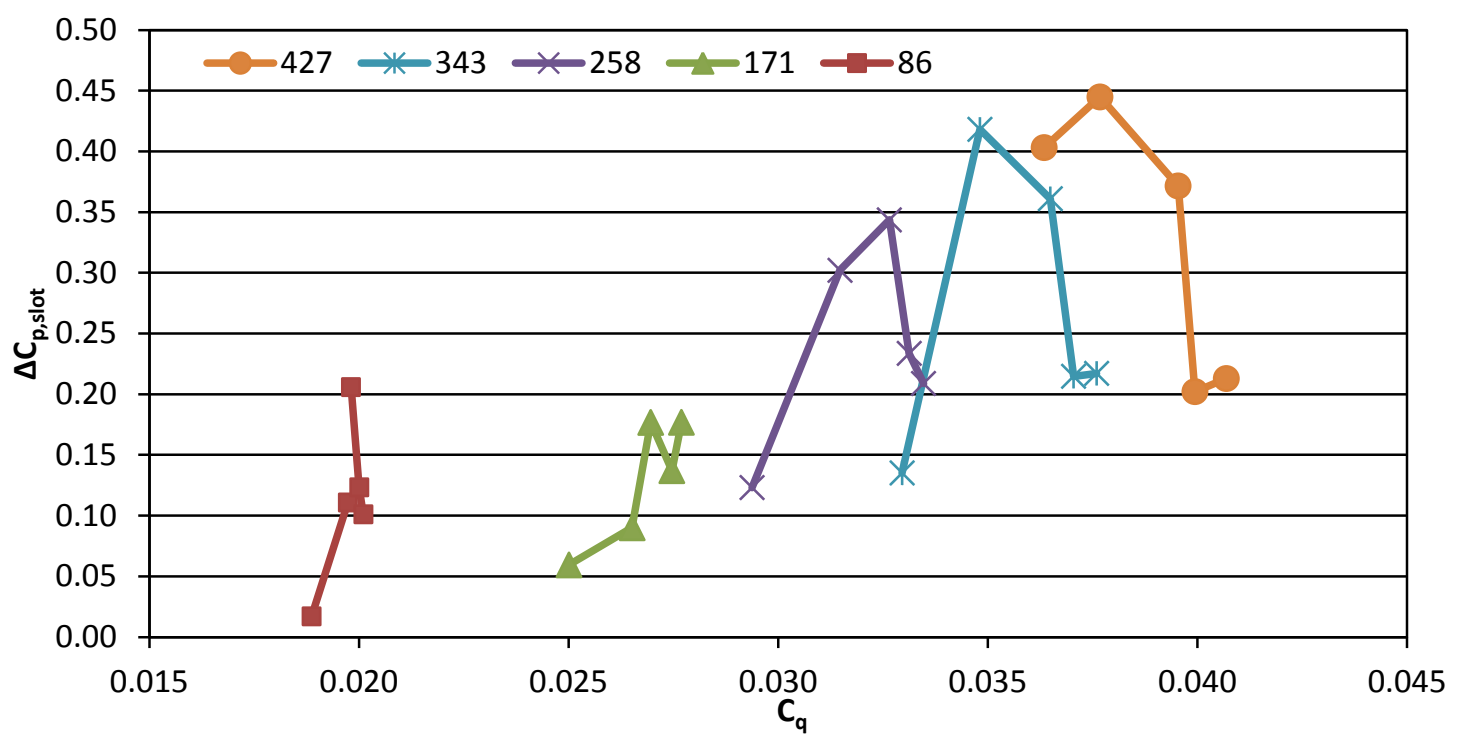

Figure 6-17. The slot pressure rise coefficient vs. suction flow coefficient for various shaft power settings.

\subsection{COMPARISON BETWEEN NO CUSP AND CUSP A}

An important start toward future testing is to begin the evaluation of different suction slot geometries. As mentioned previously, the design of the slot cusp is an important part of the Goldschmied propulsor concept. Goldschmied noted up a reduction in the suction flow rate of $66 \%$ to achieve equivalent axial force values with the addition of the Ringloeb cusp. For the current work, only one cusp was tested due to tunnel time constraints.

\subsubsection{Pressure Distribution}

The pressure coefficient distribution is shown in Fig. 6-18 through Fig. 6-23 comparing the pressure distribution with the same shaft power setting and slot gap size for the cases with and without a cusp. Overall, these plots are very clear and consistent in showing that the cusp did not appreciably effect the pressure distribution on the model for any fan power or slot gap. It was expected that the pressure distribution upstream of the slot would not be appreciably affected by the slot geometry change, but that the pressure distribution aft of the slot would be considerably changed. The cusp at the slot geometry is meant to only redirect the flow aft of the slot, thus leaving 
the upstream velocity and pressure distribution unchanged and altering the pressure distribution aft of the slot only. This was not the case. The cusp did not appreciably affect the flow aft of the slot. It largely showed the same characteristics and trends as the no-cusp cased. The error in the pressure measurements are very small, as will be discussed in Section 6.3. This resulted in a high degree of confidence that the similarities in the data sets are accurate.

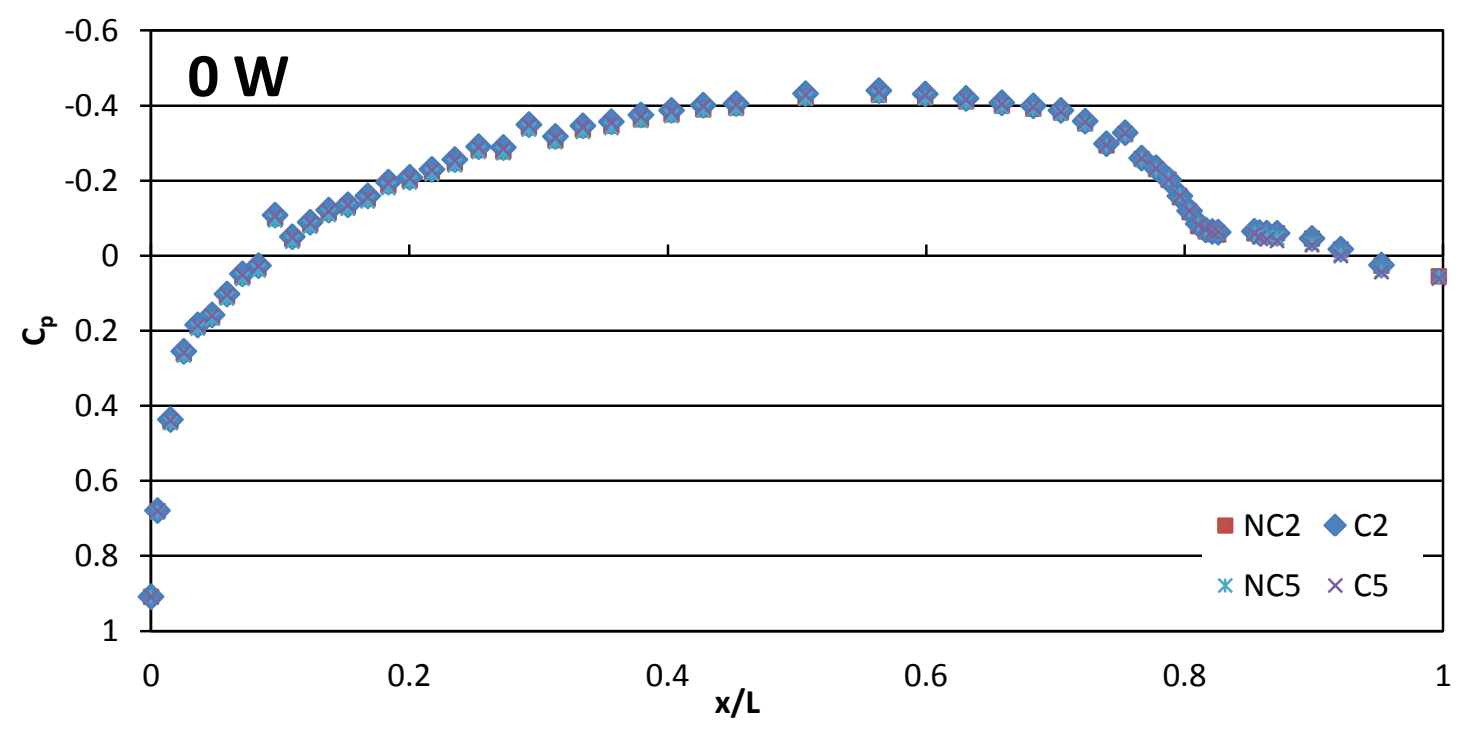

Figure 6-18. $\mathrm{C}_{\mathrm{p}}$ distribution for two slot gaps with and without a cusp and no suction. 


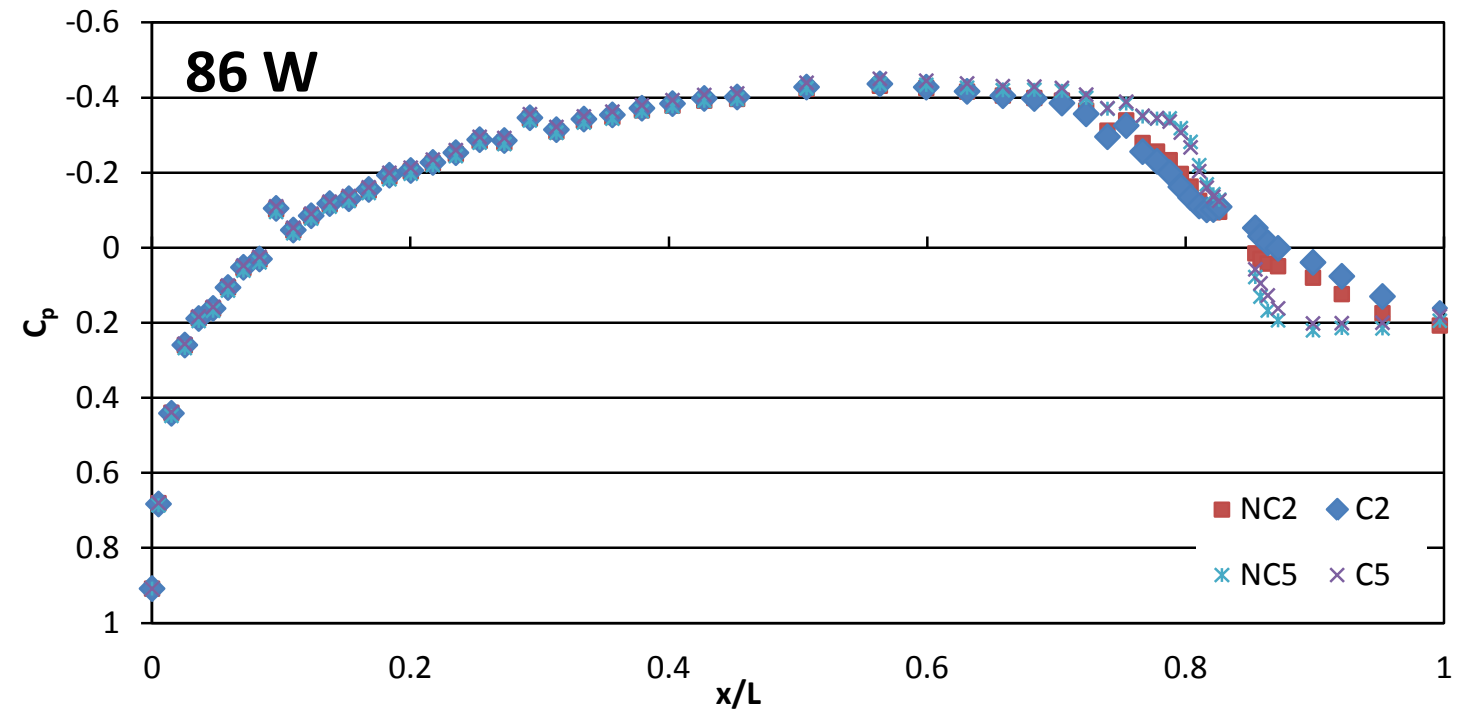

Figure 6-19. $C_{p}$ distribution for two slot gaps with and without a cusp and $86 \mathrm{~W}$ of shaft power.

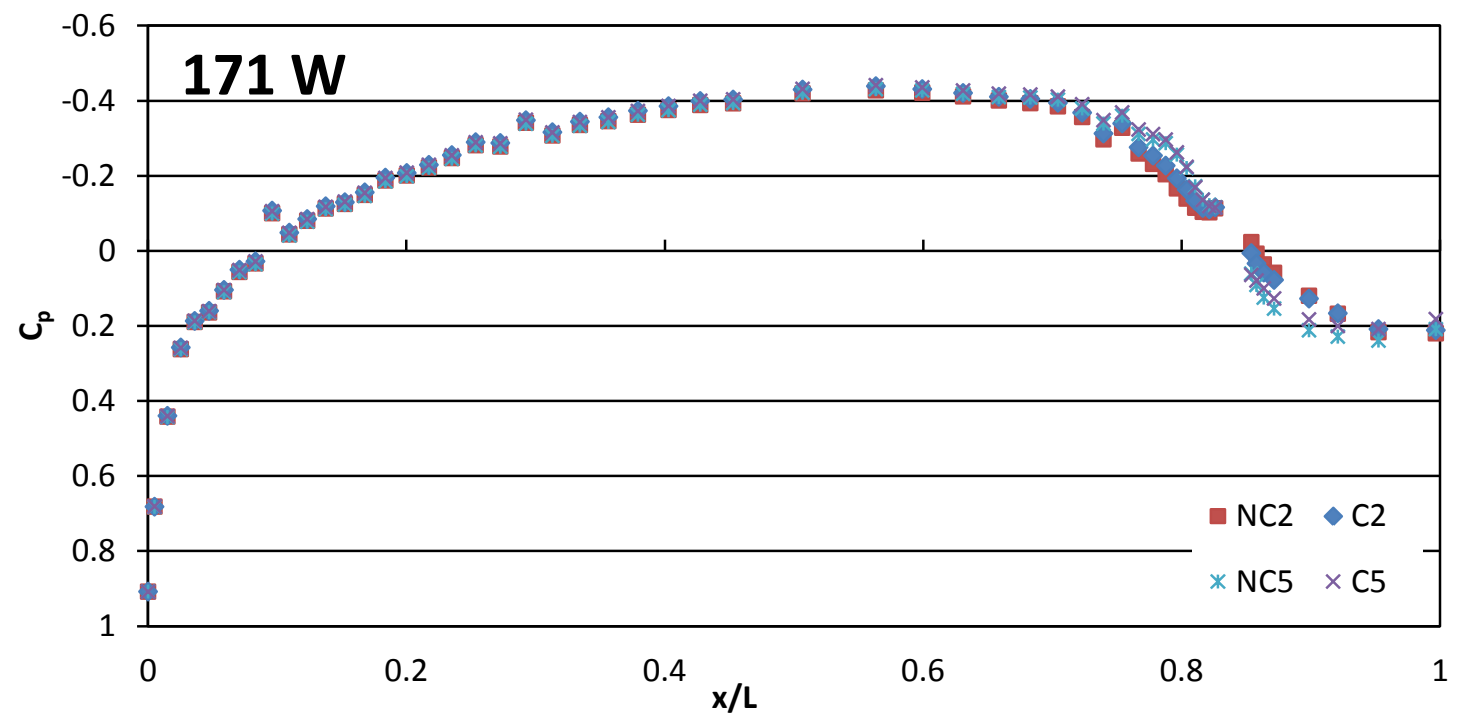

Figure 6-20. $C_{p}$ distribution for two slot gaps with and without a cusp and $171 \mathrm{~W}$ of shaft power. 


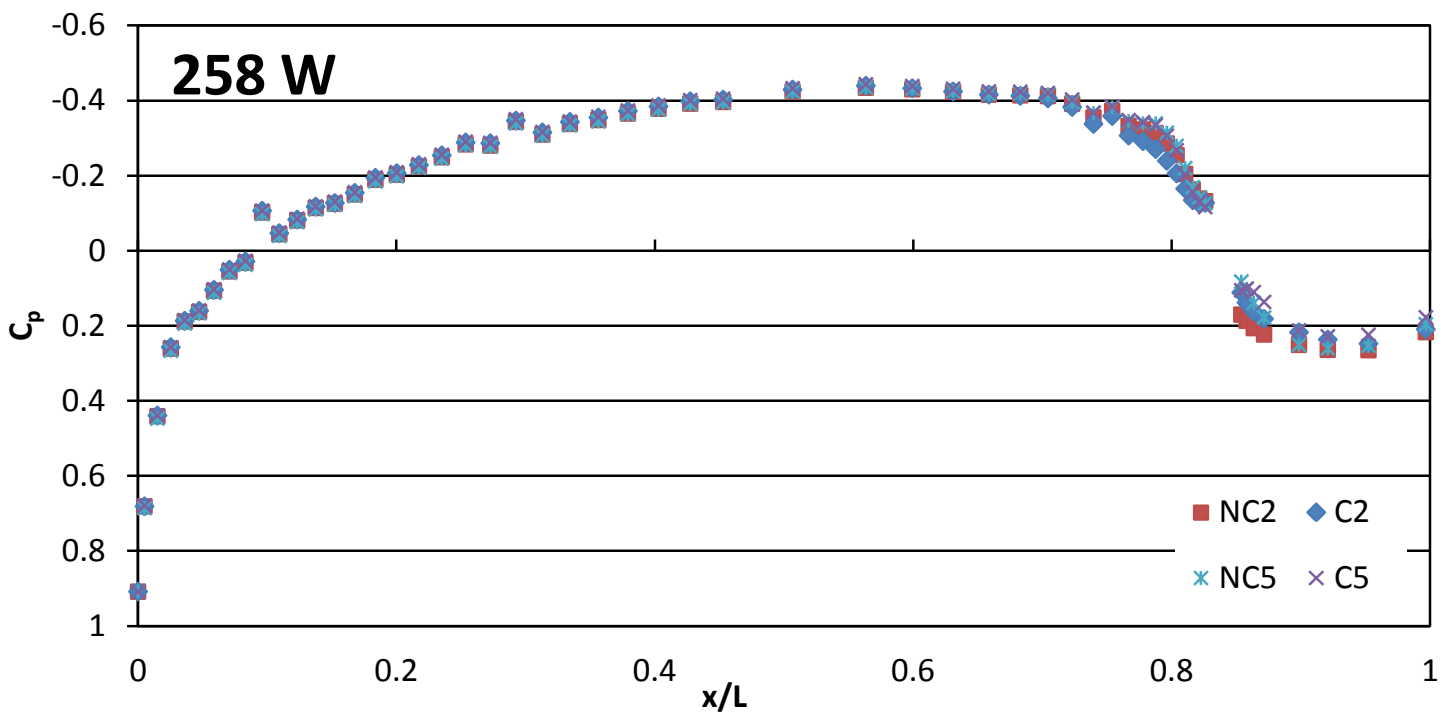

Figure 6-21. $C_{p}$ distribution for two slot gaps with and without a cusp and $258 \mathrm{~W}$ of shaft power.

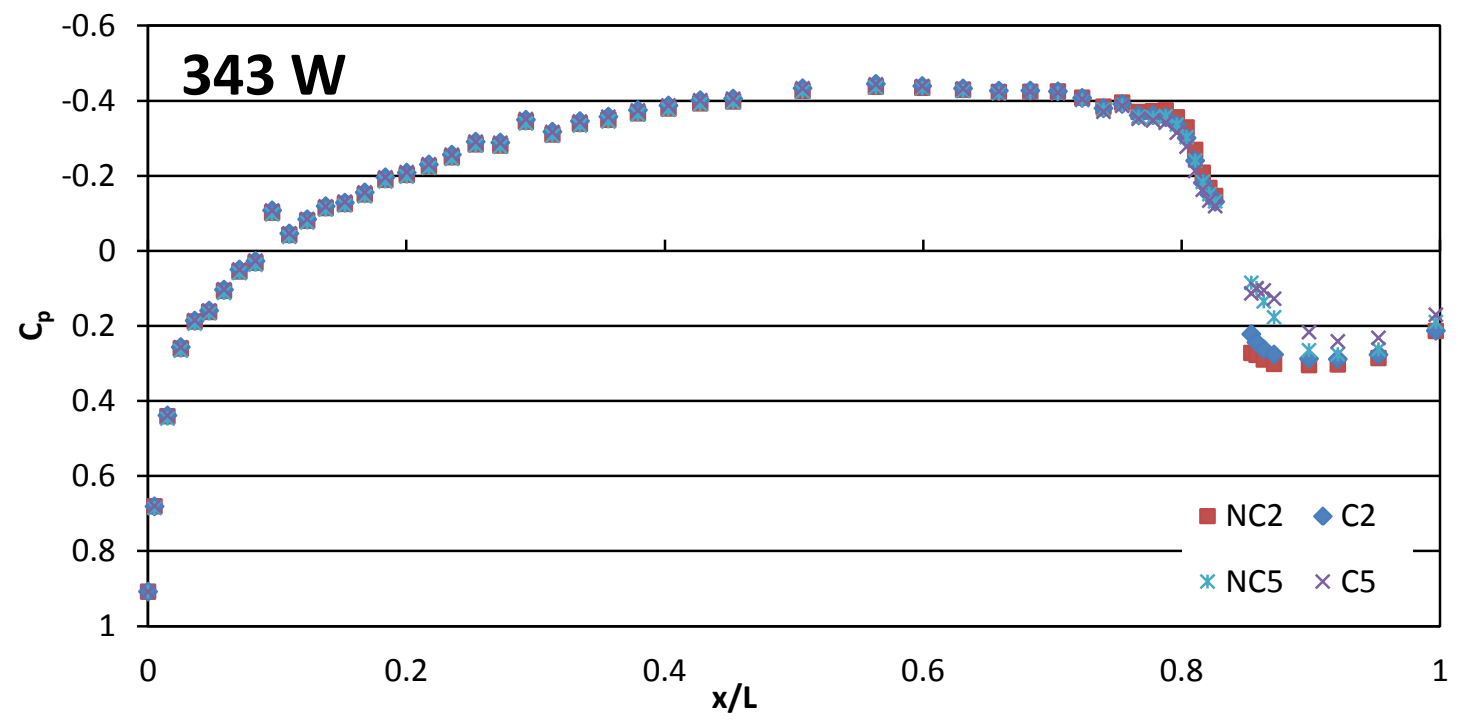

Figure 6-22. $C_{p}$ distribution for two slot gaps with and without a cusp and $343 \mathrm{~W}$ of shaft power. 


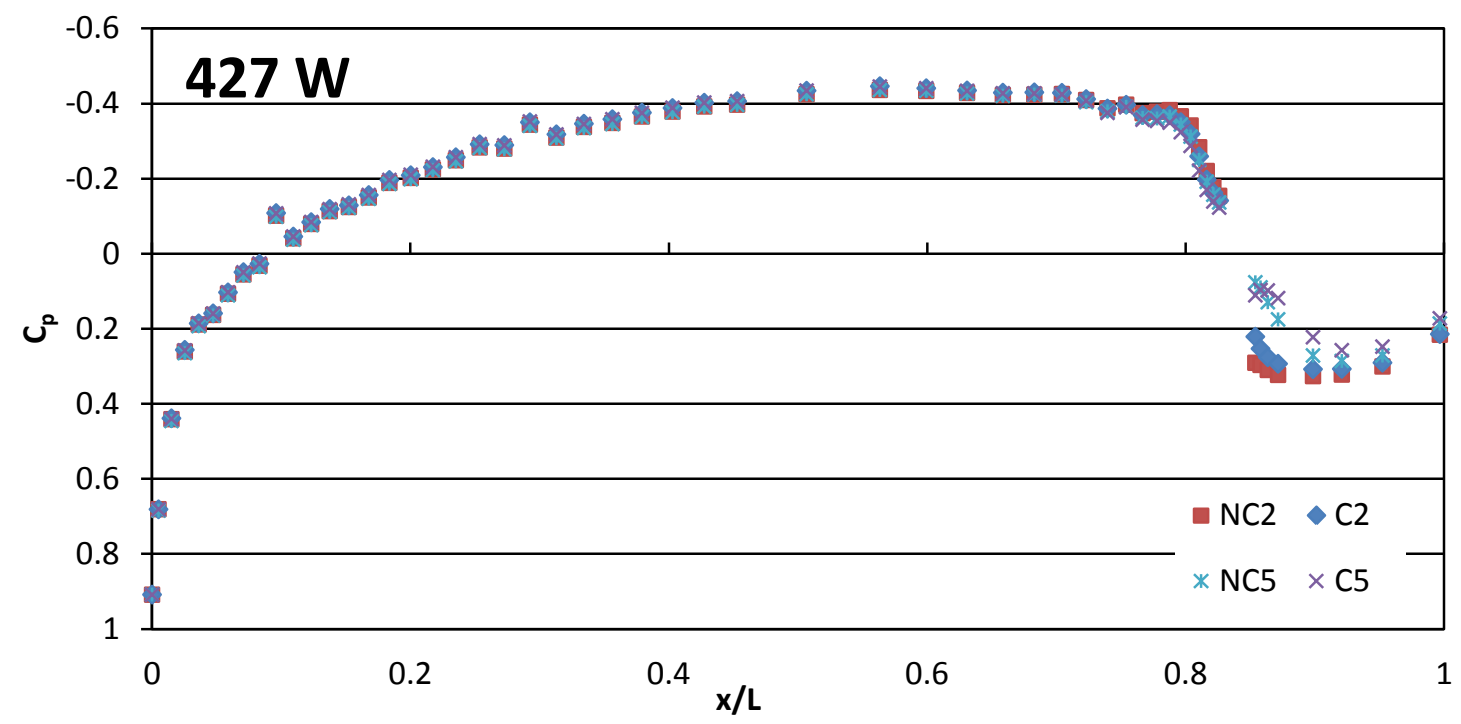

Figure 6-23. $\mathrm{C}_{\mathrm{p}}$ distribution for two slot gaps with and without a cusp and $427 \mathrm{~W}$ of shaft power.

\subsubsection{AXIAL FORCE}

The next comparison is of the axial force coefficient for the two slot geometries, as shown in Fig. 6-24. The data presented is for only three shaft power settings for each configuration in order to keep the trend apparent. The noticeable trend being that the cusp configuration shows a slightly higher, or more positive, axial force coefficient than the no-cusp data for the same power input, except at the largest slot gap. For the largest slot gap, the trend is reversed and the no-cusp configuration produced a slightly higher axial force coefficient. It is interesting that this trend was even present for the fan off, or $0 \mathrm{~W}$, case. This adds credibility to the original Ringloeb trapped-vortex concept and to Goldschmied for using the concept for the suction slot geometry.

The following average values will exclude the slot gap at $1.3 \%$ slot gap because of its large influence. For a constant shaft power of $427 \mathrm{~W}$, the average increase in $\mathrm{C}_{\mathrm{A}}$ is 0.007 . This is an increase of about $8 \%$ over the no-cusp configuration for the same shaft power. The individual data point with the largest increase in $\mathrm{C}_{\mathrm{A}}$, again not including $\mathrm{NC} 4$, is $\mathrm{C} 3$ over the NC3 case with an increase of 0.024. 


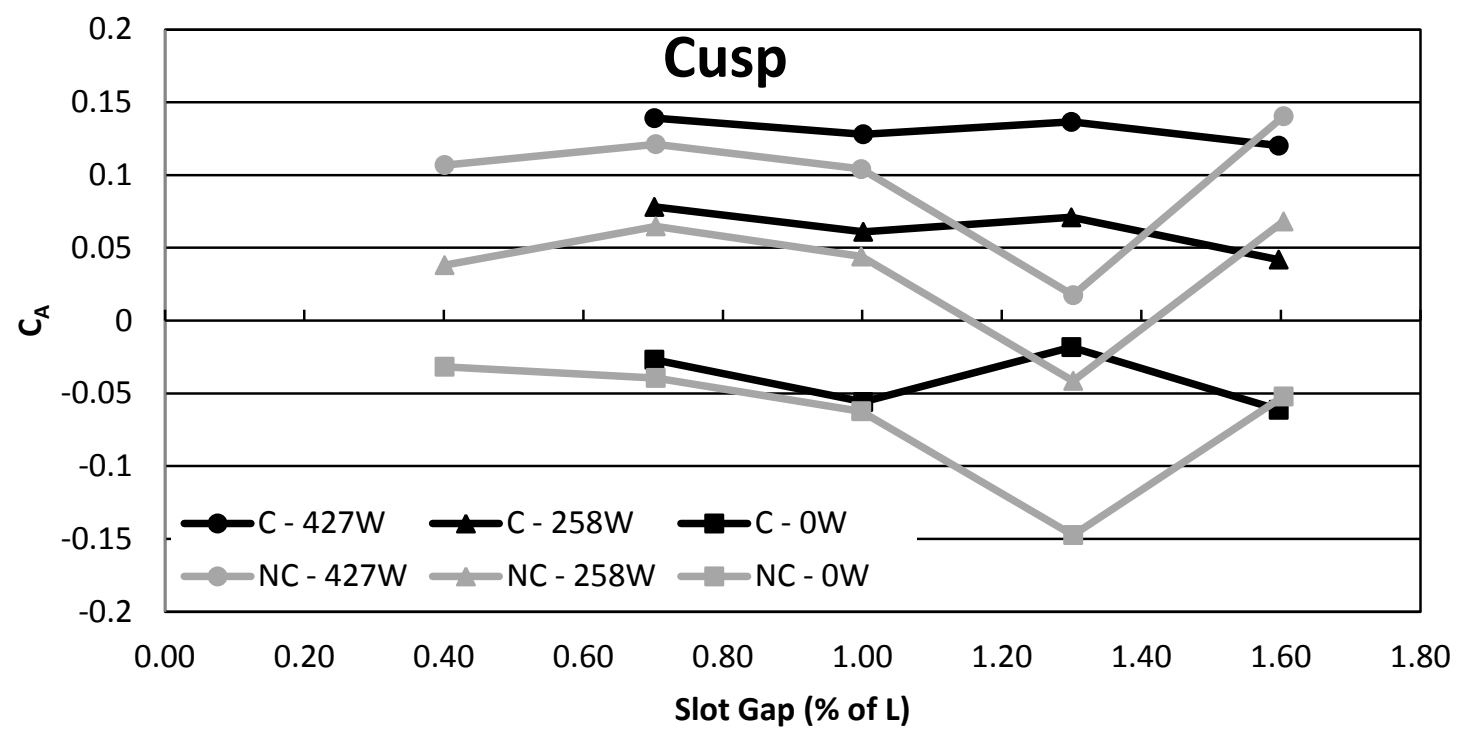

Figure 6-24. Comparison of the axial force coefficient for with and without a cusp at the suction slot.

\subsection{DATA ACCURACY}

Data accuracy is a quantification of the error associated with any given measurement. The total error can be thought of as a combination of the random error and the systematic error. As mentioned earlier, an error analysis and propagation has been completed for all parameters and all data points. It utilized the standard deviation of the mean as the estimate of the parameter's random error with a $95 \%$ confidence interval for all data points. This method resulted in very small estimates of the random error for all parameters since a large number of samples were taken for all parameters.

For channels that utilized a calibration curve in their data reduction, the systematic error is estimated by the average absolute value of the residuals that resulted from the calibration. The random and systematic error estimates can be combined in a number of ways to estimate the total error in the parameter. In Table 6-2, both types of error are shown for select parameters. 
Table 6-2. Summary of select test parameters and their corresponding error estimates.

\begin{tabular}{|c|c|c|c|c|c|}
\hline Parameters & Units & $\begin{array}{c}\text { Random Error } \\
\text { Range }\end{array}$ & $\begin{array}{c}\text { Ave. Random } \\
\text { Error }\end{array}$ & $\begin{array}{c}\text { Systematic } \\
\text { Error Est. }\end{array}$ & $\begin{array}{c}\text { Absolute Range of } \\
\text { Parameter }\end{array}$ \\
\hline Axial Force & Lbs & $0.0008-0.0040$ & 0.0015 & 0.142 & $-0.840-0.805$ \\
\hline $\begin{array}{c}\text { Axial Pressure } \\
\text { Force Coefficient }\end{array}$ & Unit-less & $0.00096-0.00163$ & 0.0012 & 0.014 & $-0.0202-0.0108$ \\
\hline $\begin{array}{c}\text { Suction Mass } \\
\text { Flow Rate }\end{array}$ & Slugs/s & $0-1.84 \times 10-5$ & $1.39 \times 10-5$ & $\mathrm{n} / \mathrm{a}$ & $0-0.00667$ \\
\hline Fan Current & Amps & $0.010-0.021$ & 0.017 & 0.048 & $0-29.97$ \\
\hline Fan Thrust & Lbs & $0.097-0.924$ & 0.604 & 0.077 & $0-1.369$ \\
\hline Fan Torque & In-Lbs & $0.61-5.54$ & 2.72 & 0.18 & $0-73.38$ \\
\hline Slot Gap & Inch & $1.6 \times 10-5-2.2 \times 10-5$ & $1.9 \times 10-5$ & 0.0026 & $0.152-0.688$ \\
\hline
\end{tabular}

Another estimate of the total error is to repeat some of the data points and determine the difference in the measurements. This gives not only an estimate of the error in a particular parameter, but it gives an estimate of the error that is due to variations in the control parameters. The latter of which is usually referred to as repeatability. The variation in the fan shaft power control parameter is estimated to be $\pm 8 \mathrm{~W}$, and the variation in the slot width control parameter is estimated to be \pm 0.007 '. The variation in the shaft power is due to the slow power supply current increases while recording data due to the increased motor temperature. The slot gap tolerance is the result of the variation in the slot gap over the circumference of the slot. Setting the slot gap was usually accurate to less than 0.002 '

The ability to repeat a given model configuration and have the measured parameters be able to be very close to their previous values is an indication that the systematic error in the system is low, and that the major influences on the experiment's measured parameters is taken into account and controlled. This section will address the repeatability of the measurements. As mentioned in Section 5.1, nine data points from the first half of the test matrix, the half without a cusp, were repeated for this purpose. The pressure distribution is presented first, followed by the total axial force.

Since the pressure measurements' calibration process is completed at the sensor manufacturer's facility, the systematic error due to the calibration process is known and is very low. In general, the random error estimate, while 
being very low, was higher than the systematic error by 1-2 orders of magnitude. This resulted in very low total error estimates for all the pressure measurements, which give high confidence in these data sets.

To estimate the repeatability of the measurements, the pressure distributions for the original and repeated data are shown in Fig. 6-25 through Fig. 6-28. In general, they show very good repeatability between the two data sets, especially for the NC2, NC3, and NC5 configurations. The NC4 configuration showed good agreement for all cases except for the $171 \mathrm{~W}$ case. This case shows better pressure recovery on the aftbody and more negative pressure on the forbody forward of the suction slot. The repeat run shows the characteristics of a run with higher shaft power.

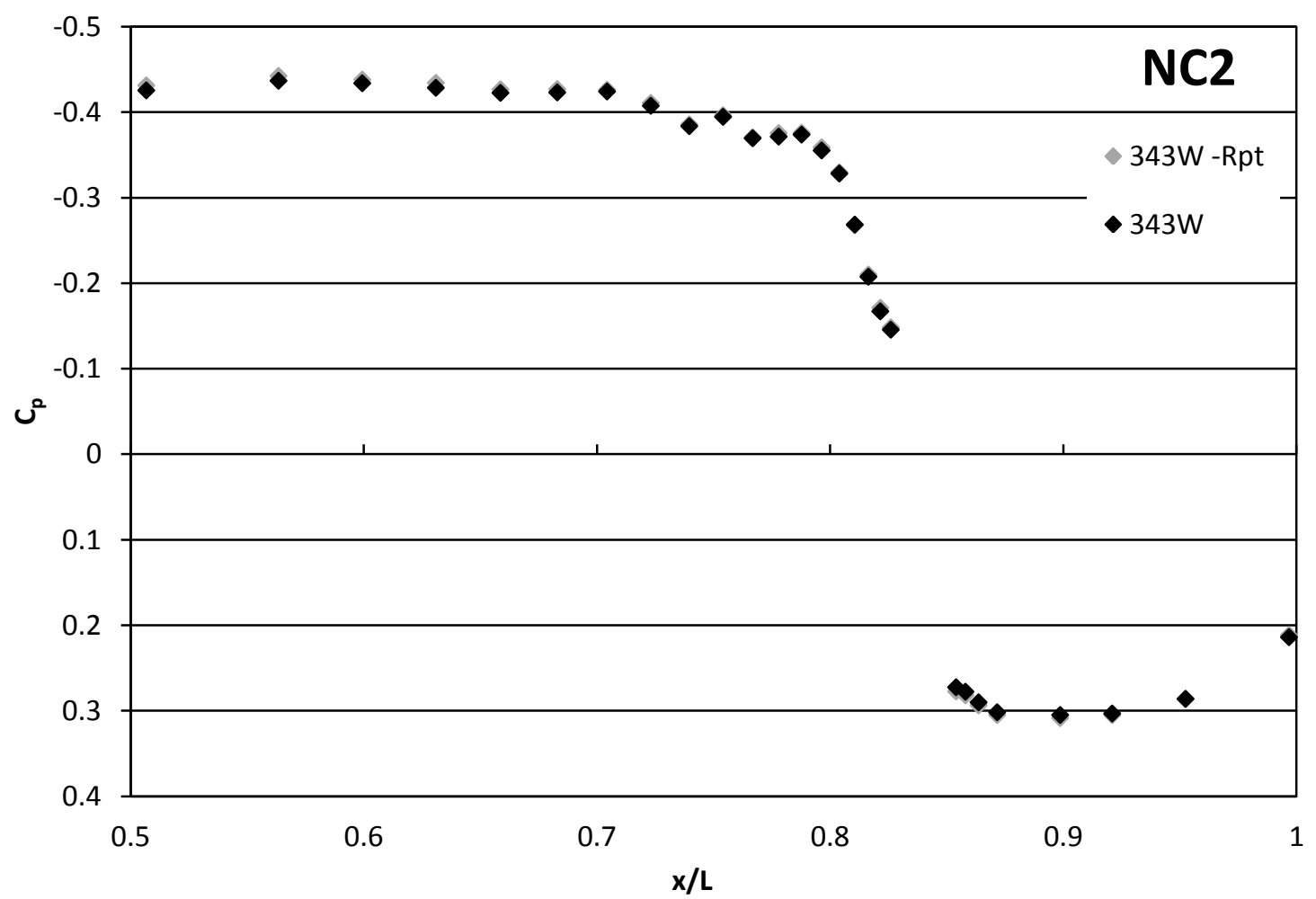

Figure 6-25. $\mathrm{C}_{\mathrm{p}}$ distribution for the $\mathrm{NC2}$ configuration with $343 \mathrm{~W}$ of shaft power comparing the original data and data taken later on in test matrix. 


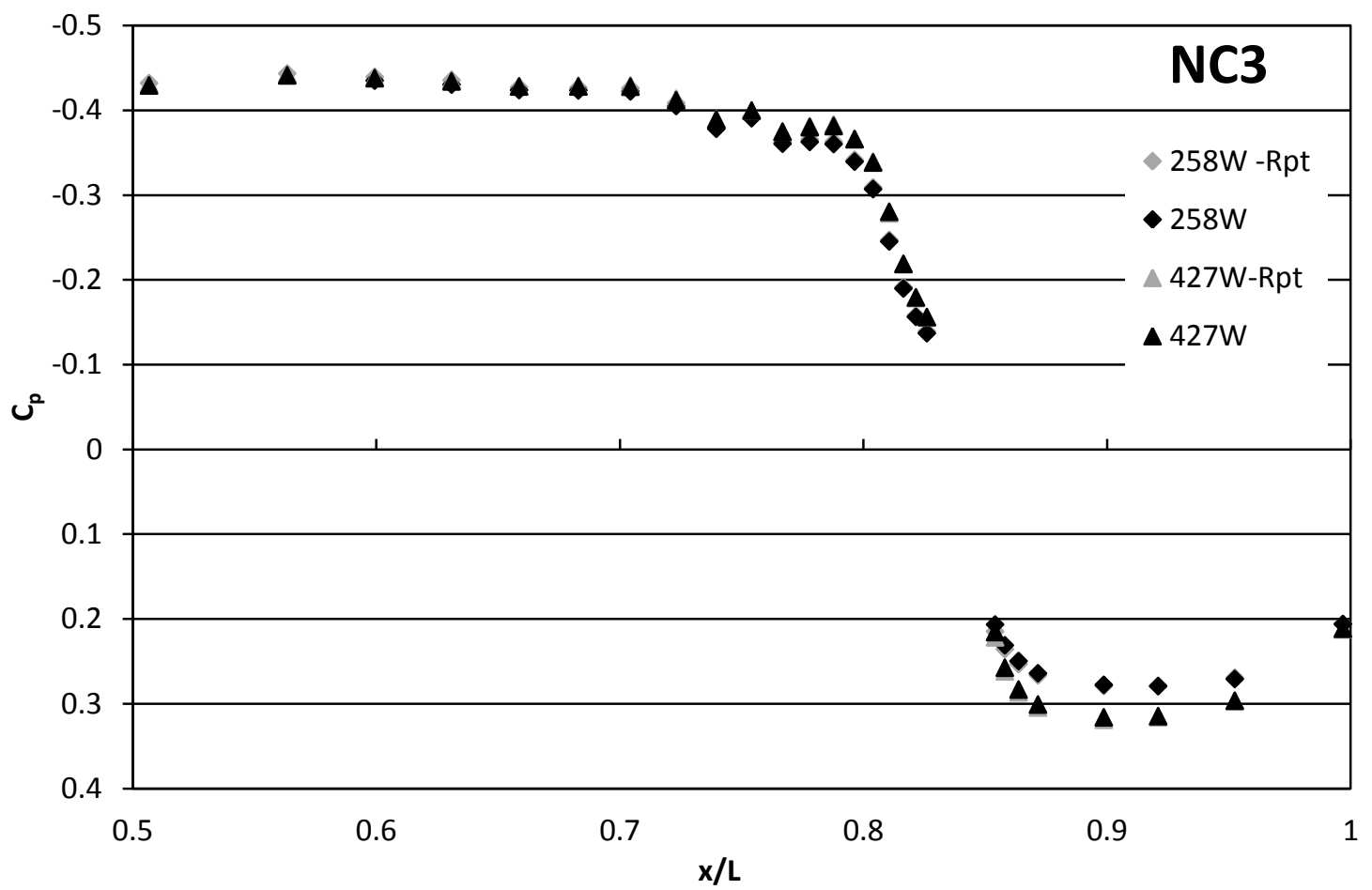

Figure 6-26. $\mathrm{C}_{\mathrm{p}}$ distribution for the $\mathrm{NC3}$ configuration with $258 \mathrm{~W}$ and $427 \mathrm{~W}$ of shaft power comparing the original data and data taken later on in test matrix.

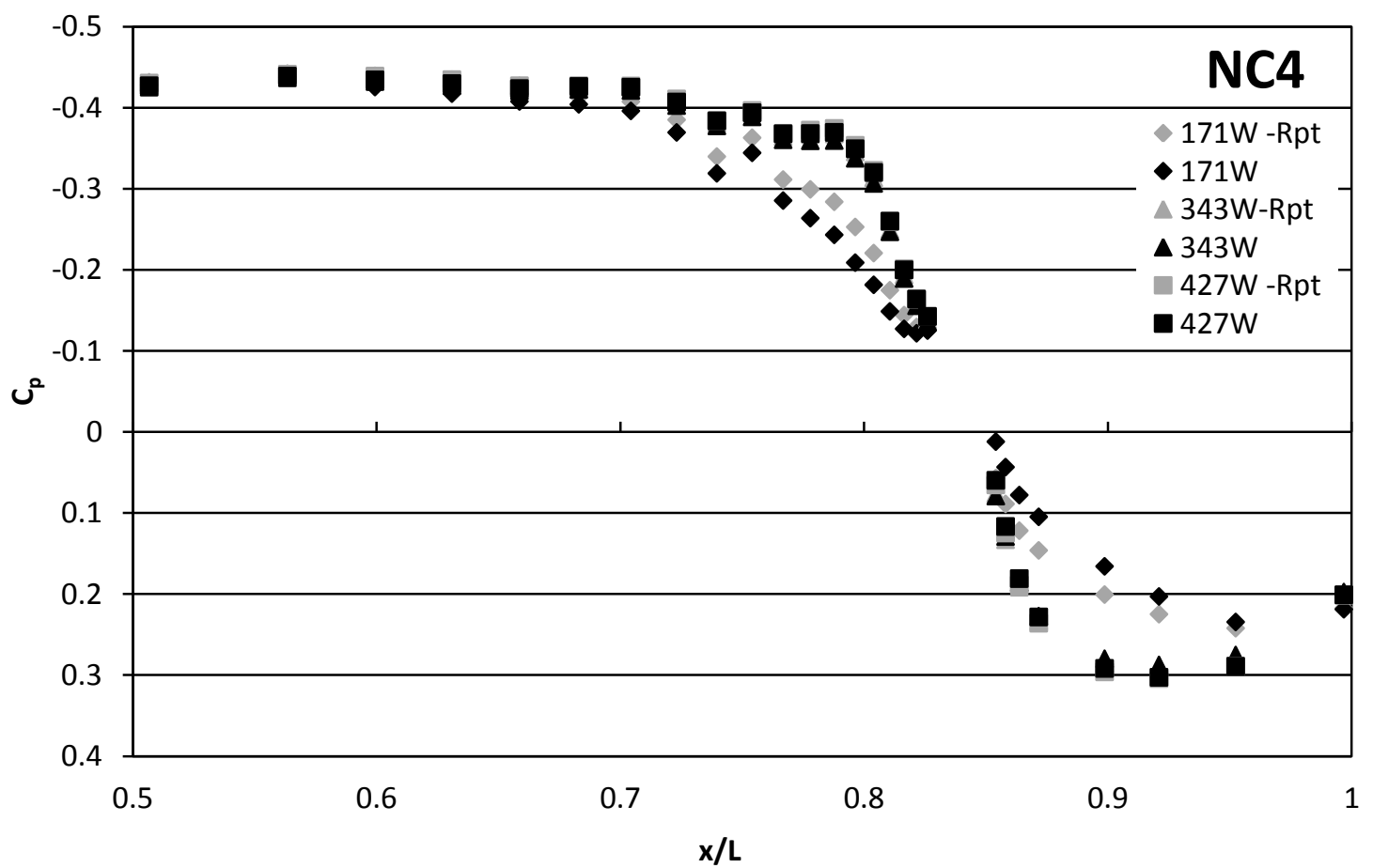

Figure 6-27. $\mathrm{C}_{\mathrm{p}}$ distribution for the $\mathrm{NC3}$ configuration with $171 \mathrm{~W}, 343 \mathrm{~W}$, and $427 \mathrm{~W}$ of shaft power comparing the original data and data taken later on in test matrix. 


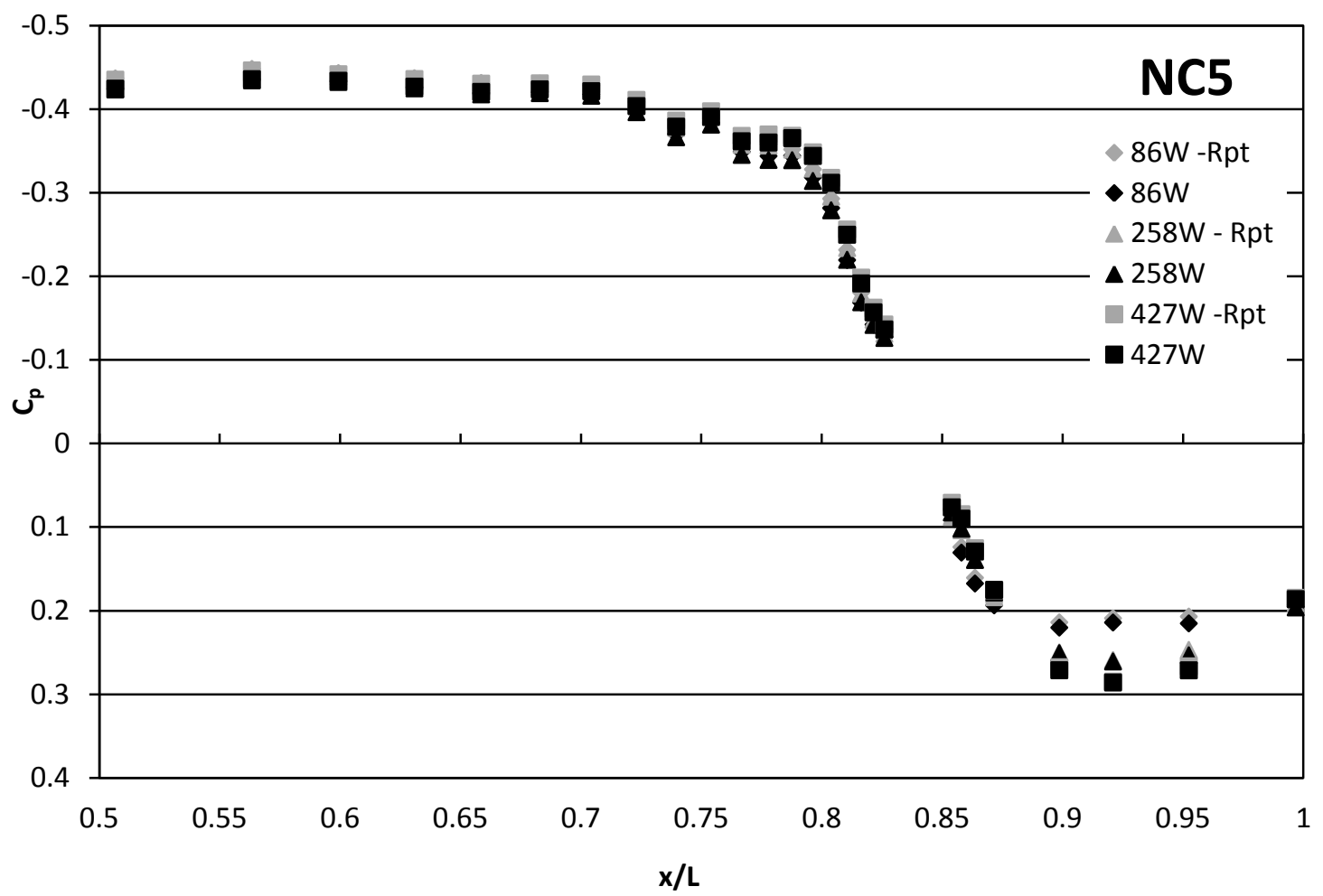

Figure 6-28. $\mathrm{C}_{\mathrm{p}}$ distribution for the $\mathrm{NC3}$ configuration with $86 \mathrm{~W}, 258 \mathrm{~W}$, and $427 \mathrm{~W}$ of shaft power comparing the original data and data taken later on in test matrix.

The above pressure distributions indicate that the flow fields around the model were accurately reproduced when the data points were repeated. This indicates that, for most data points, the test points were repeatable and with small systemic error in the pressure measurements.

The total axial force measurements, on the other hand, are not as reproducible. The total axial force is shown in the Fig. 6-29 through Fig. 6-33 for various shaft power settings and slot widths. In general, the repeat data points do not show good agreement with the original data points. The exception to this is at the largest slot gap where the repeat points are within 0.0088 of the original points for all shaft power settings.

Besides the fact that the axial force is not repeatable in general, the distinctly lower axial force seen at all shaft power settings at a slot gap of $1.3 \%$ is definitely not a true representation of the real data. This was not reproduced in any of the three repeat data points that examined it. Something was influencing the measurements and/or the model when the original data points were being measured that was not being taken into account by the test operator. After examination, this anomaly does not show up in any of the other measured parameters. During 
testing, the test log did not show any extraordinary events, and all data points were taken sequentially with no breaks or model changes except to change the slot gap and fan power. The current theory that explains the incorrect measurements is that the motor efficiency varies significantly more than expected with motor temperature. This would result in the shaft power applied to the fan being considerably different from expected. This would also explain the non-repeatability of the other data points, and not just the NC4 configuration. The data that supports this theory is that the motor was very cool for the NC5 data points, and then was considerably hotter by the time the NC4 data points were run, and then stayed about this temperature for the rest of the no-cusp data points. The motor temperature data does not really support or disprove this theory, and should be evaluated further in any future testing.

The repeat data shows a definite shift toward higher axial force. For the NC5 case, the shift is between $0.0002-0.0088$. For the NC4 case the shift is in the range of $0.132-0.137$. For NC3 it is between 0.055 and 0.058 . The only repeat point at NC2 was 0.045 higher than the original point. This shows a definite offset that was caused by something that was not controlled by the test operator.

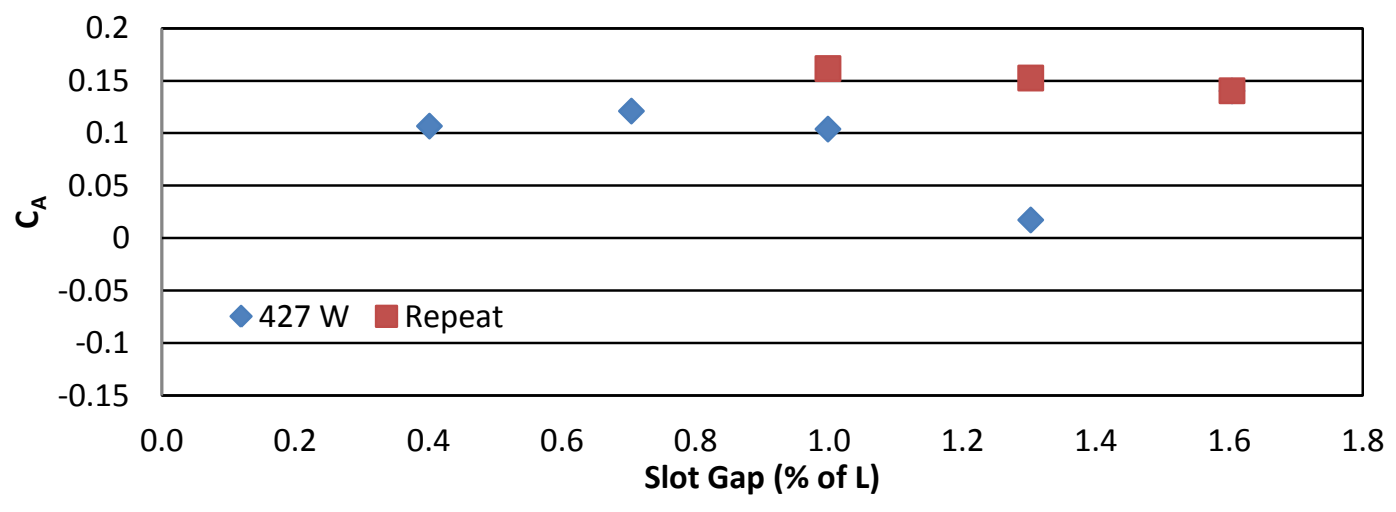

Figure 6-29. Total axial force as a function of slot gap for $427 \mathrm{~W}$ of shaft power comparing the orginal data and select repeated points to evaluate the precision of the measurements. 


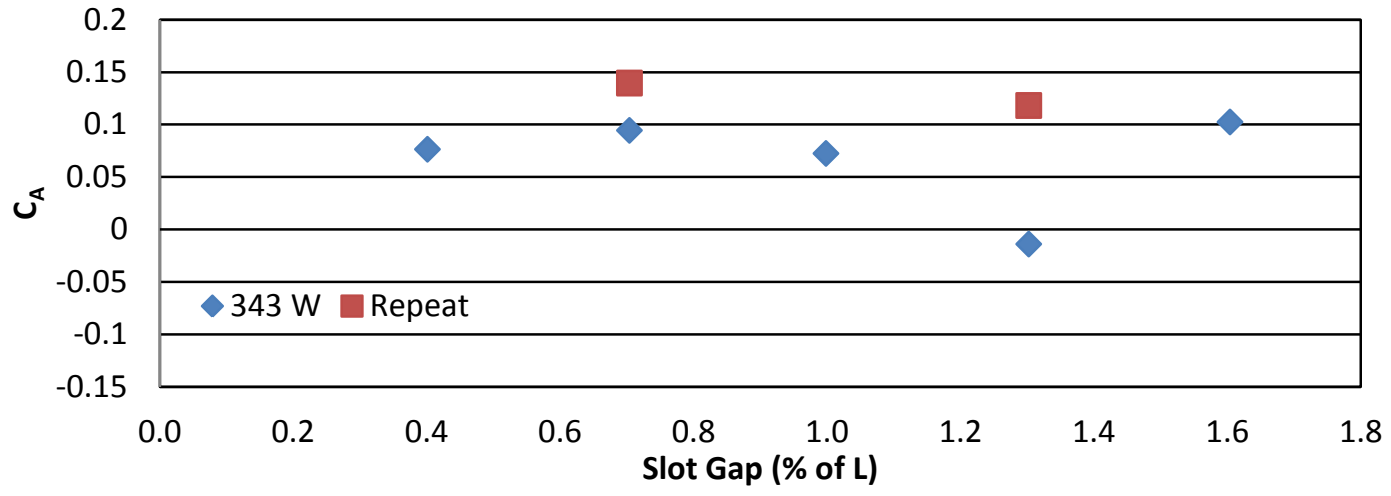

Figure 6-30. Total axial force as a function of slot gap for $343 \mathrm{~W}$ of shaft power comparing the orginal data and select repeated points to evaluate the precision of the measurements.

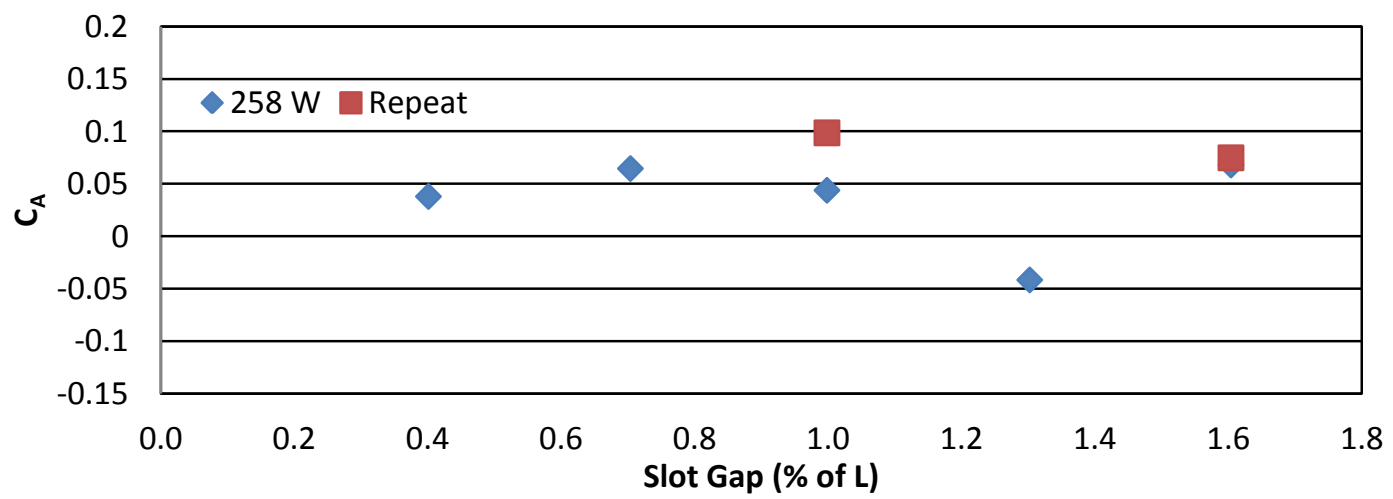

Figure 6-31. Total axial force as a function of slot gap for $258 \mathrm{~W}$ of shaft power comparing the orginal data and select repeated points to evaluate the precision of the measurements.

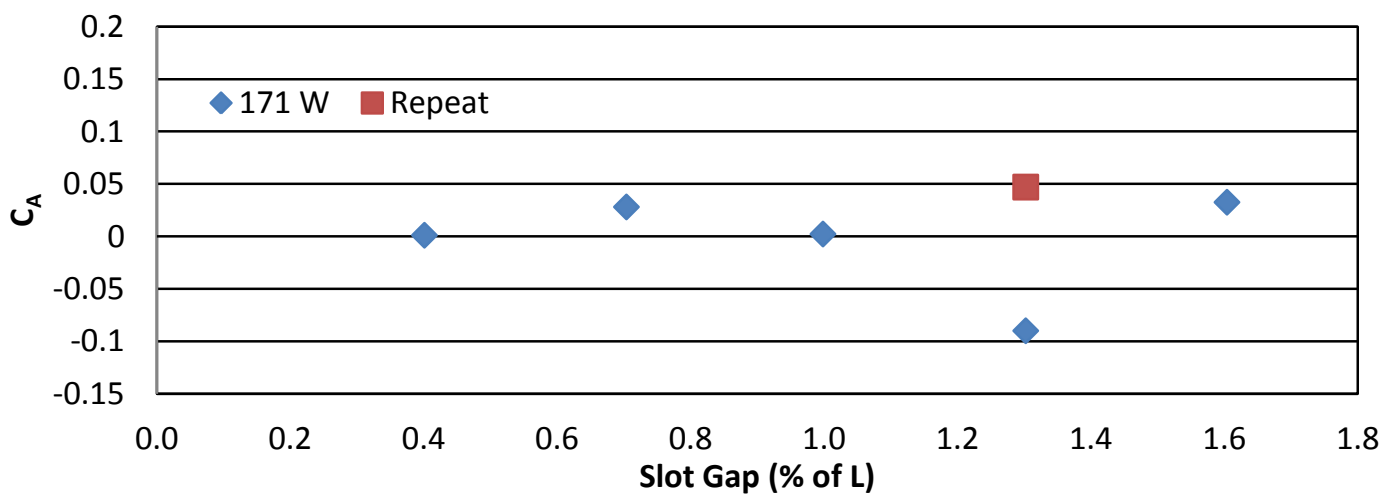

Figure 6-32. Total axial force as a function of slot gap for $171 \mathrm{~W}$ of shaft power comparing the orginal data and select repeated points to evaluate the precision of the measurements. 


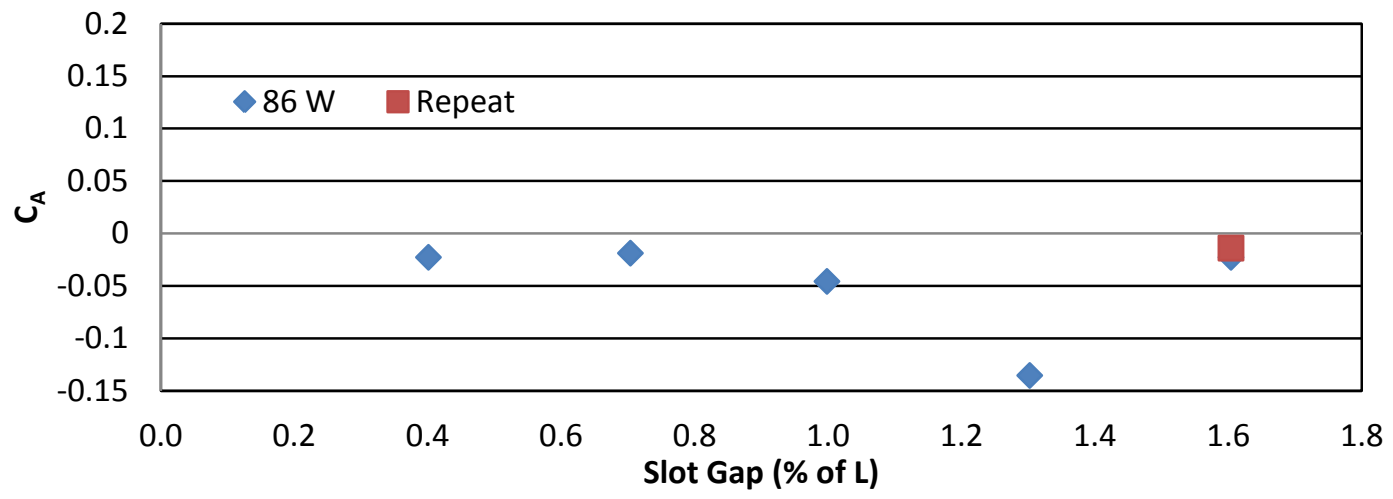

Figure 6-33. Total axial force as a function of slot gap for $86 \mathrm{~W}$ of shaft power comparing the orginal data and select repeated points to evaluate the precision of the measurements.

The above figures demonstrate a problem with the axial force measurements. On average, excluding the NC4 data, the average error is 0.030 . In percentage of the original axial force measurements, the error ranges between $24 \%$ and $139 \%$ of the original data, with an average value of $75 \%$. The total axial force coefficient will not be emphasized very strongly in the rest of this paper due to this high degree of uncertainty.

\subsection{FAN PERFORMANCE}

The fan performance was a large unknown during the design phase of this experiment. The fan manufacturer did not publish any performance data and they could not provide it when requested. So for future reference and future efforts with the current model, the fan performance is presented. A carpet plot of the flow rate and fan pressure rise for various shaft power and slot gaps is presented in Fig. 6-34. The solid lines are lines of constant slot gap and varying shaft power, while the dashed lines are constant shaft power and varying slot gap. From the shape of the $427 \mathrm{~W}$ line of constant power, it is evident that this fan is operating at a part of the fan performance curve that is not ideal for efficient operation. The non-monotonic behavior indicates that the fan could suffer from unsteady behavior when operated in this region. The unsteadiness stems from the minima in the curve. When the fan is operated close to this point, the flow rate could either increase or decrease and the pressure difference across the fan will increase. This behavior was not specifically noticed during the testing, but it is something to be aware of for any future testing programs. 


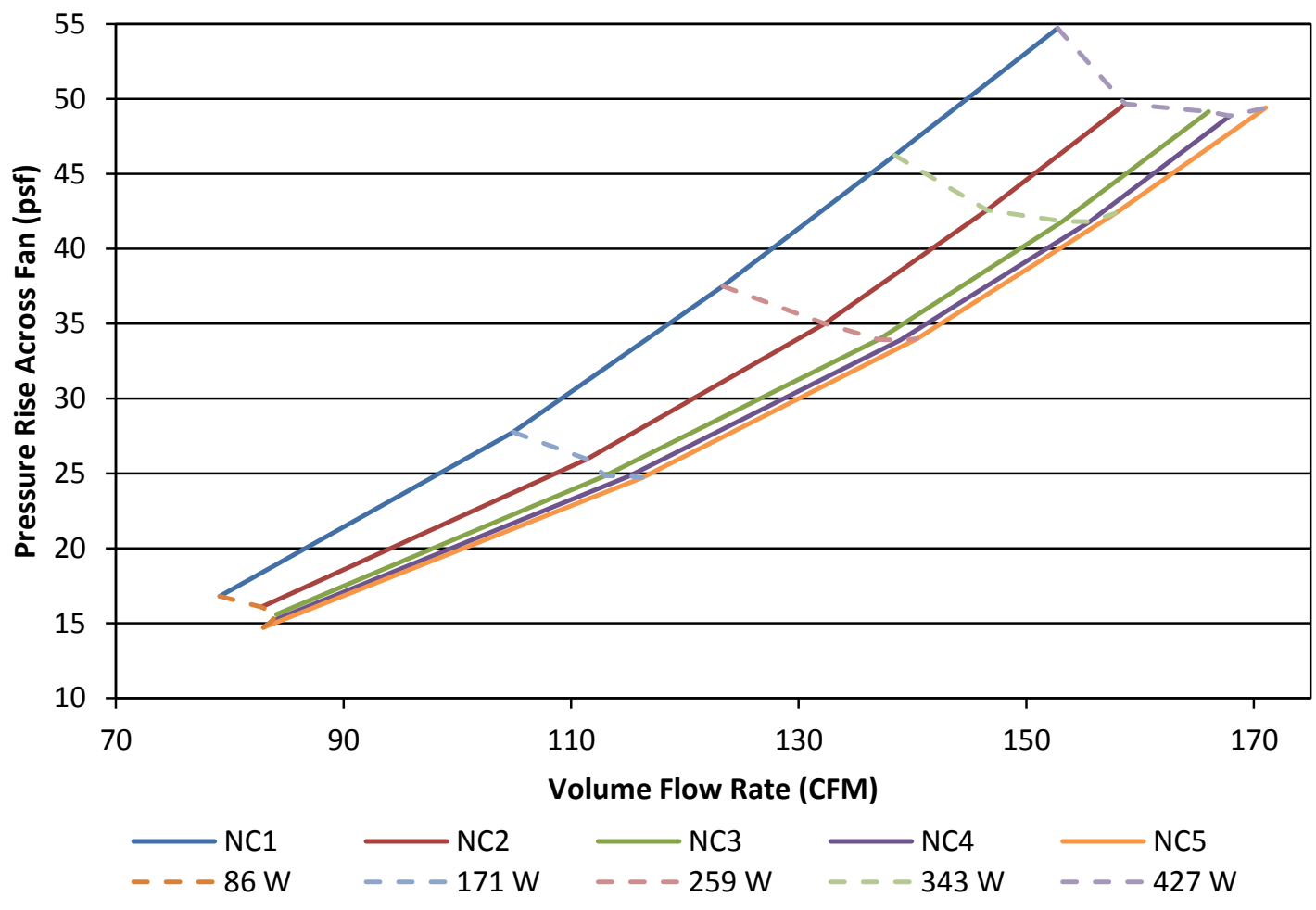

Figure 6-34. Fan performance of the Hacker B40-11L and Schubeler DS30 fan installed in the NGP

The relationship between fan speeds and the fan power, thrust, and pressure rise show very traditional trends. The fan shaft power, thrust, and pressure rise show nearly linear relationships with fan speed. These are shown in Fig. 6-35 through Fig. 6-36.

Unfortunately, one of the load cells that measures fan torque broke during the initial flow visualization procedure before the test matrix was even started. This breakage could not be fixed because all spare load cells had been already used to fix previous breakages. The many breakages of these load cells were because they have a rated capacity of $1 \mathrm{lb}$ each, which was often exceeded during testing. Unfortunately, without this parameter, the fan output power and fan efficiency cannot be presented. 


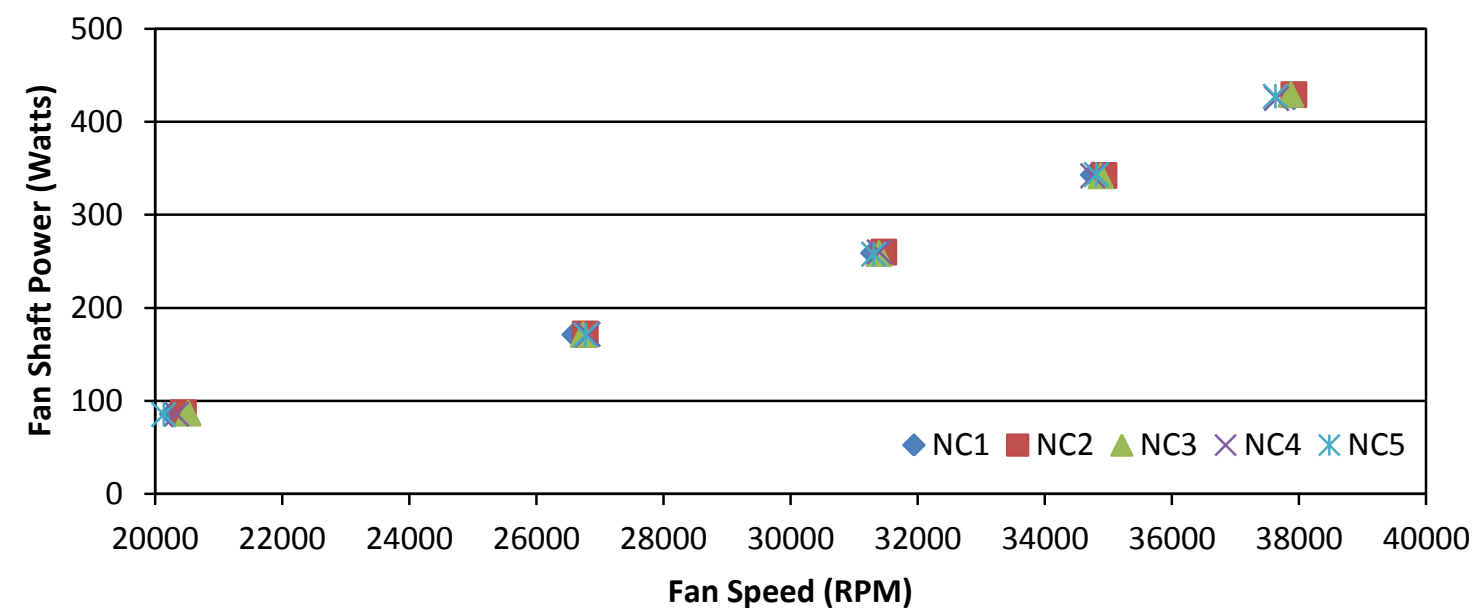

Figure 6-35. Fan rotational speed vs. shaft power of the Hacker B40-11L and Schubeler DS30 fan installed in the NGP

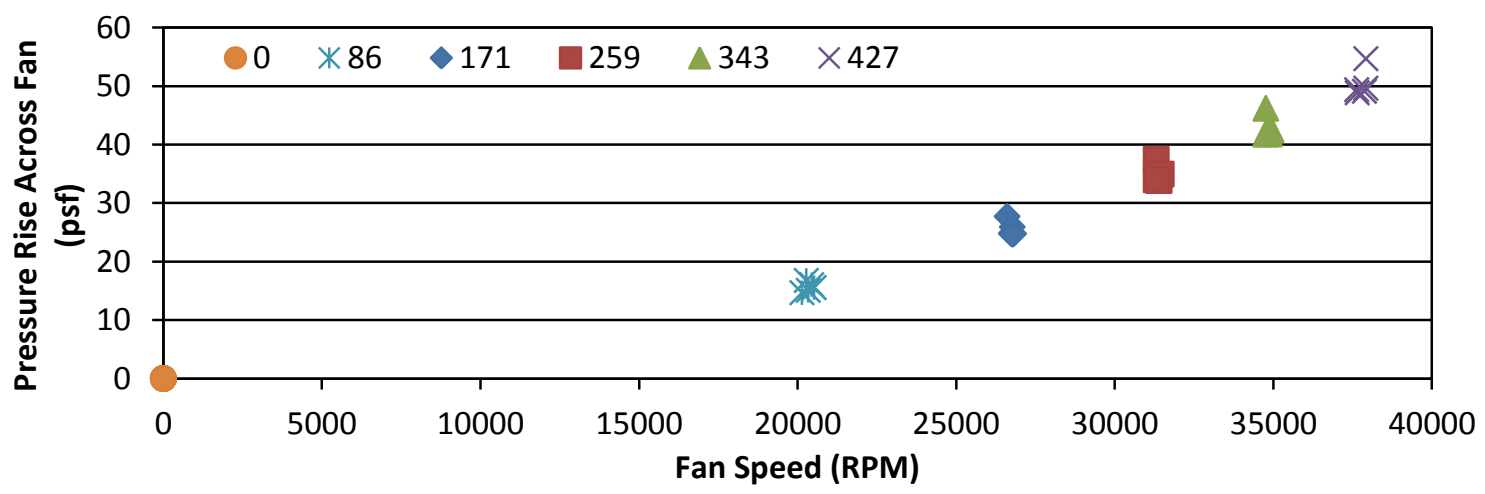

Figure 6-36. Fan rotational speed vs. fan pressure rise for the Hacker B40-11L and Schubeler DS30 fan installed in the NGP

The fan thrust is shown in Fig. 6-37and Fig. 6-38. The former is as a function of fan speed. This shows a simple linear or mildly quadratic relationship between the two parameters. The latter figure shows the measured fan thrust as a function of the fan thrust derived using something similar to what would be derived using actuator disk theory for the fan. The pressure rise across the fan times the swept area of the fan gives a force. By comparing these two parameters, it provides a check of the quality of the measurement of fan thrust. Ideally, the fan trust would equal the calculated thrust. In this case, the signal's slope is 0.991 , and the offset is about $0.5 \mathrm{lbs}$. The data is linear enough to have an $\mathrm{R}^{2}$ value of 0.989 . This offset could be caused by friction between the fan duct and the fan inlet, or it 
could be caused by interference between the load cells and the wiring, or tubing in the cramped confines of the aftbody.

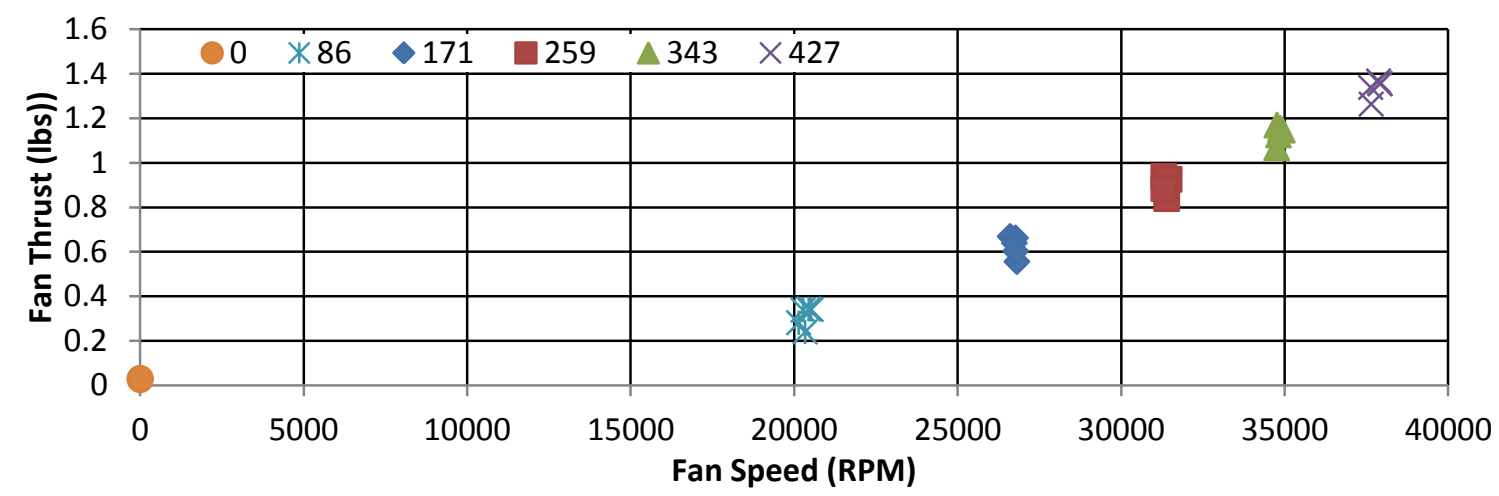

Figure 6-37. Fan rotational speed vs. fan thrust of the Hacker B40-11L and Schubeler DS30 fan installed in the NGP

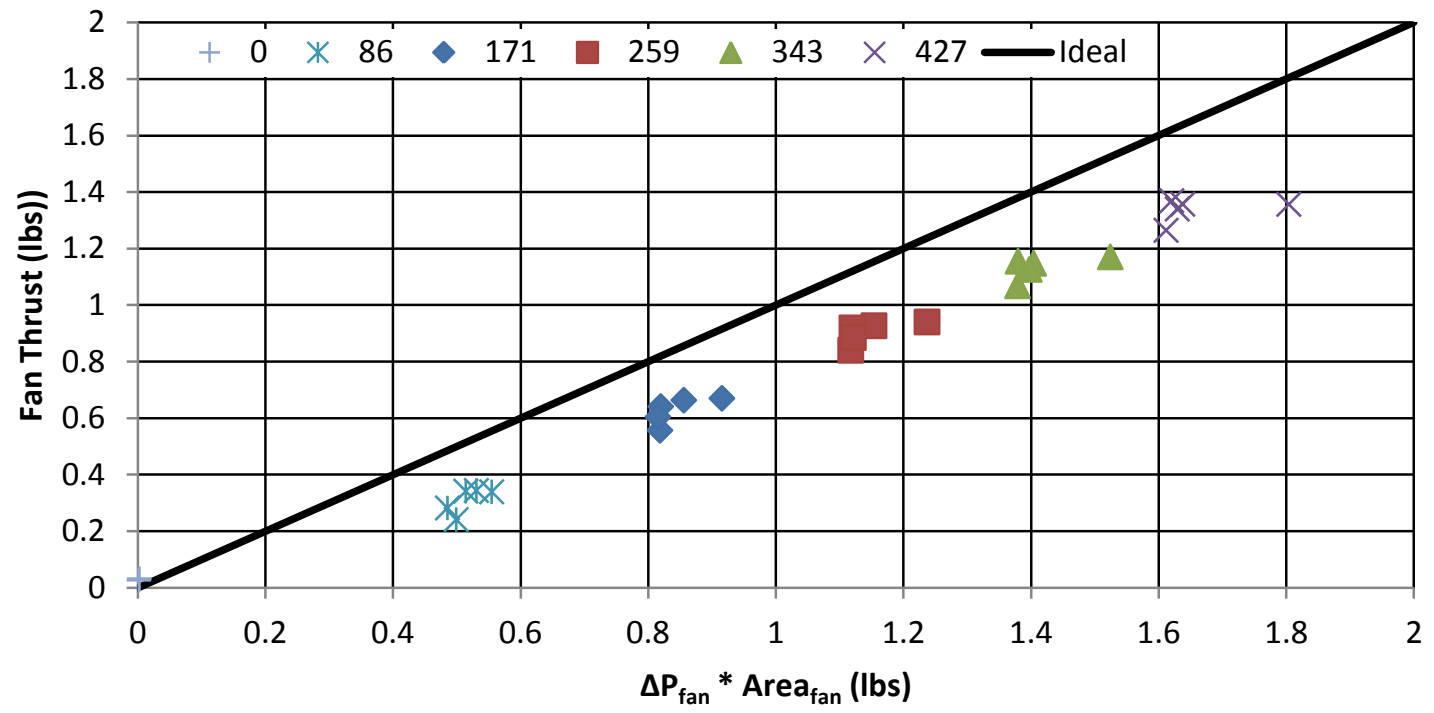

Figure 6-38. The measured fan thrust, from the load cells, vs. the fan thrust calculated from the fan pressure rise and the fan swept area.

\subsection{COMPARISON TO PREVIOUS TEST DATA}

Just as important as the comparison between the data points with and without a cusp is the comparison to previous Goldschmied tests. This comparison bridges the gap between the past and present testing efforts. Even though the models are different in some key aspects, they are fundamentally the similar. 
The Reynolds number for the current test is about 1/3 the lowest Reynolds number from the previous Goldschmied tests. The best available data to compare to is from the original 1956 Goldschmied test. The pressure data for the test is difficult to read in the available reports, but the set of data for a similar Reynolds number that could be found for a no-suction and suction case are presented in Fig. 6-39 and Fig. 6-40 for comparison. Overall, the data sets only show slight agreement. This is mainly due to the difference in Reynolds number between the cases, which are about a factor of three different.

For both cases, the pressure coefficient on the forbody for the 1956 test is slightly lower over the entire forbody. This offset is not indicative of a Reynolds number effect. The Reynolds number effect would cause the pressure coefficient to be more positive in the areas of the forbody where the pressure coefficient is positive and more negative where the pressure coefficient is negative. This is not the case.

In addition, the NGP could not achieve as much pressure recover on the aftbody compared to the 1956 test with suction. This could be attributed to the slight differences in suction slot geometry. The diameter at the suction slot of the current model is slightly larger than originally intended in order to add the adapter plate for the cusps. This adapter plate is faired into the originally intended body with wax. This wax and adapter plate is a possible cause of this reduced aftbody pressure recovery.

For the no-suction case, the pressure coefficient is more negative everywhere on the body. The difference is an average of about 0.12 between the data sets. One interesting feature of the original data from 1956 is the pressure coefficient on the entire aftbody was slightly positive for zero suction. This means that the surface pressure was higher than the local static pressure. For the current test data set, only the last two data points were positive. This could point to a difference between the processing of the data because a positive $C_{p}$ on the entire aftbody in the separated wake with no cusp or suction seems a little optimistic. In addition, the pressure gradient on the aftbody of the 1956 data is slightly favorable, whereas the data for the current test is slightly adverse, as would be expected for no suction flow. The offset could be caused by a different way of accounting for the tare of the surface pressure data, which was originally taken on manometers. It could also be caused by the change in the separation point due to the higher Reynolds number of the 1956 test data. The separation point could be moved aft due to a similar phenomenon to a sphere in sub-critical Reynolds number flow vs. supercritical flow. 


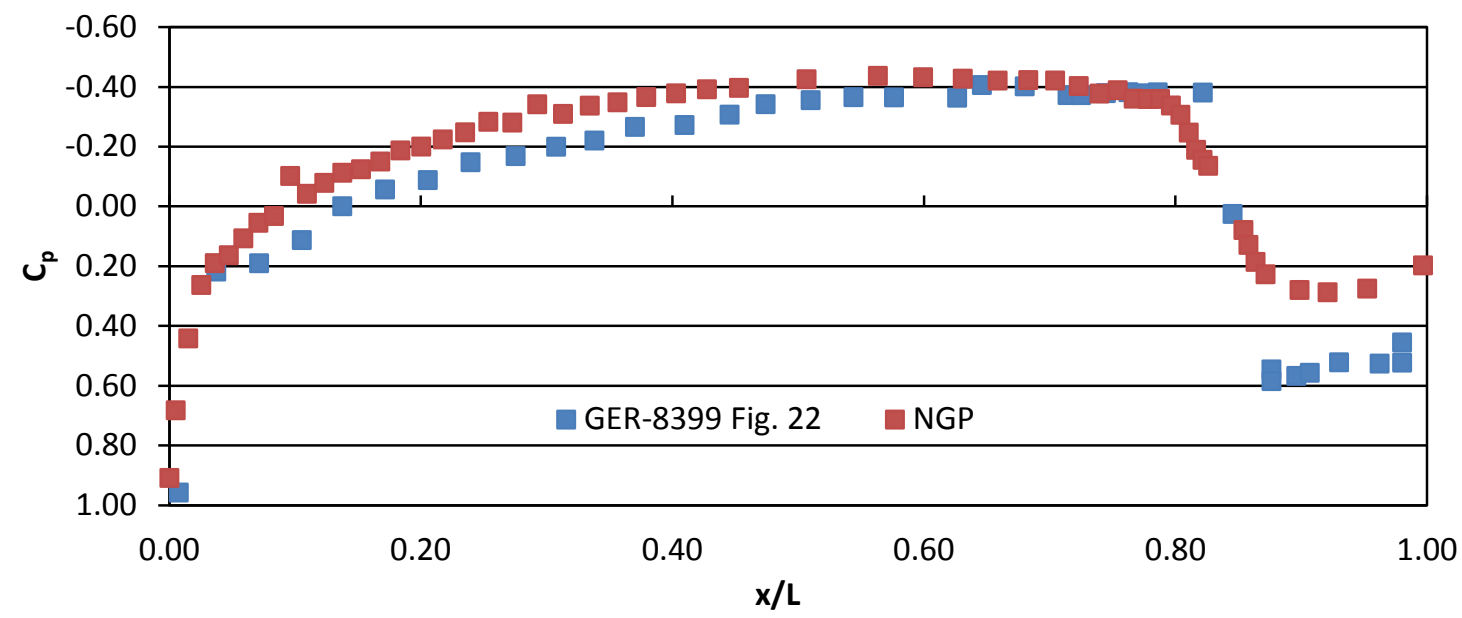

Figure 6-39. Comparison of $C_{p}$ distribution. GER-8399" is for $\operatorname{Re}_{\mathrm{L}}$ of $4.4 \times 10^{6}$ and $\mathrm{g} / \mathrm{L} \mathrm{of} 1.2 \%$ and "medium suction." NGP data is for $\mathrm{g} / \mathrm{L}$ of $1.3 \%$ and $427 \mathrm{~W}$ shaft power and a $\operatorname{Re}_{L}$ of $1.34 \times 10^{6}$.

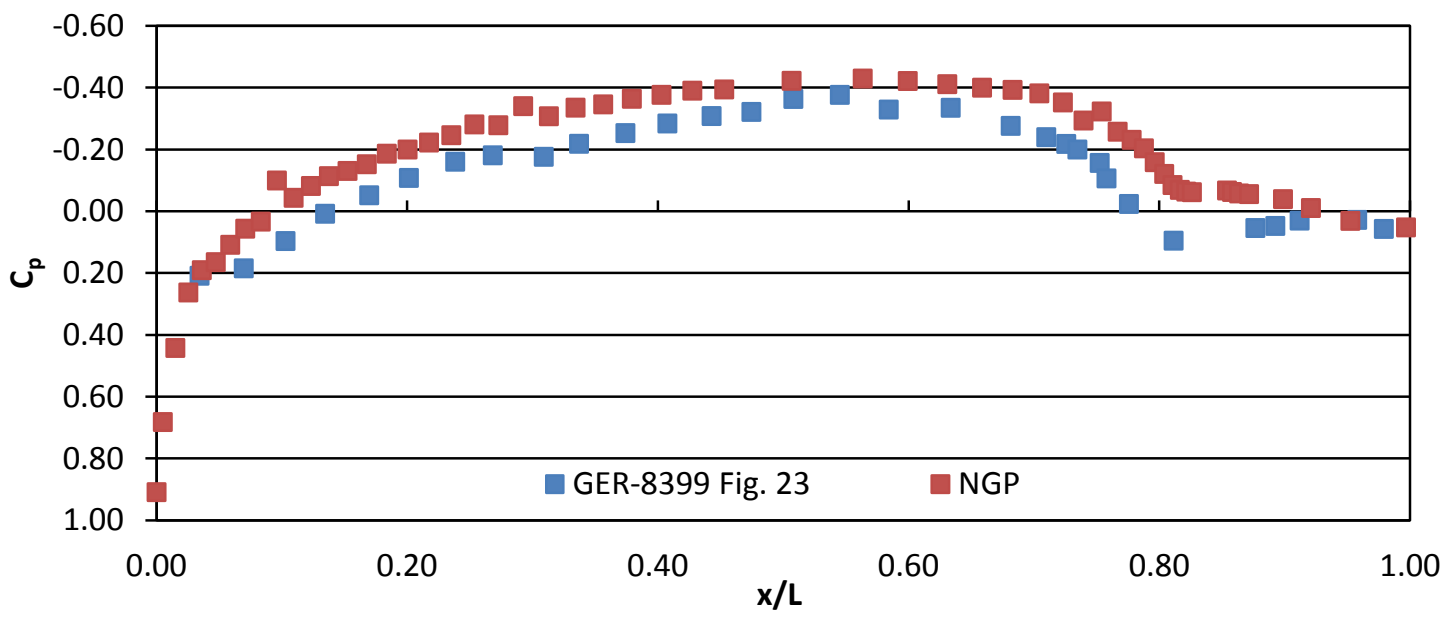

Figure 6-40. Comparison of $\mathrm{C}_{\mathrm{p}}$ distribution. GER-8399 ${ }^{4}$ is for $\operatorname{Re}_{\mathrm{L}}$ of $7.1 \times 10^{6}$ and $\mathrm{g} / \mathrm{L}$ of $1.2 \%$ and no suction. NGP data is for $\mathrm{g} / \mathrm{L}$ of $1.3 \%$ and $0 \mathrm{~W}$ shaft power and a $\operatorname{Re}_{\mathrm{L}}$ of $1.34 \times 10^{6}$.

\subsection{POWER COMPARISON}

The power comparison is an important output of the experiment. The comparison is shown in Fig. 6-41 between the NPG and a conventional aircraft, using the equations outlined in Section 0. The assumptions for the conventional aircraft are that the drag coefficient does not change as a function of shaft power, the drag coefficient is the same for both vehicles at $0 \mathrm{~W}$, and the traditional aircraft has a propulsive efficiency of $40 \%$. This value is estimated to be that from a relatively inefficient turbojet engine with an exit velocity four times the freestream velocity. 
This figure shows that the NGP requires more shaft power to achieve the same axial force coefficient, and therefore it does not show a benefit over a conventional aircraft configuration. At a propulsive efficiency of $23 \%$ is when both vehicles demonstrate very similar axial force coefficients for similar input shaft power.

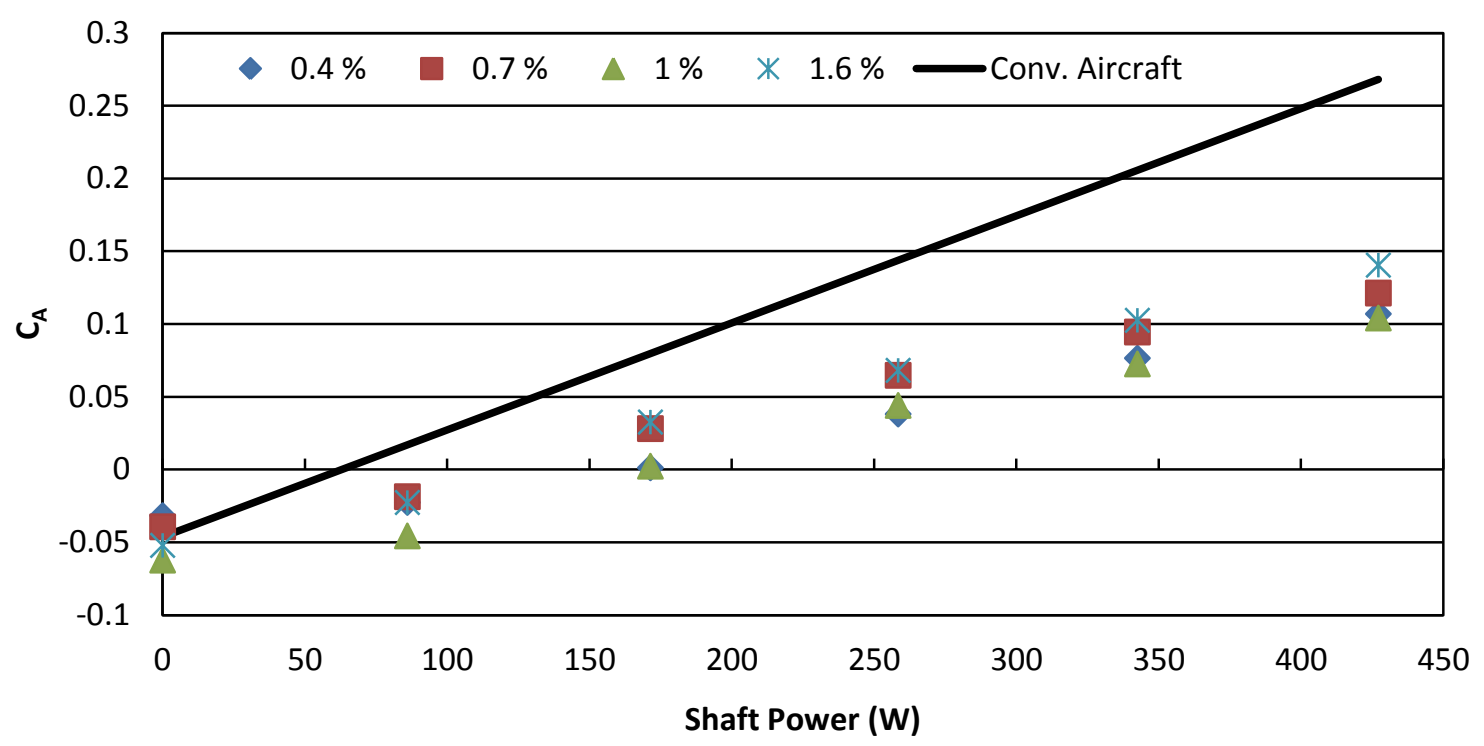

Figure 6-41. Comparison between the axial force vs. shaft power relationship of a conventional propulsor an aircraft and for the NGP

The above comparison does not include any induced drag effects for either the traditional aircraft or the NGP. To include these effects would require the inclusion of a considerable amount of assumptions. Things like the design weight, aspect ratio of the wings, and aerodynamic efficiency would all have to be assumed. The exclusion of the induced drag effects is also justified because it could be assumed that for a given flight condition, they would be very similar for both aircraft and would thus cancel each other out. If they were included, it would simply shift both data sets down into lower values of net axial force. The important comparison here is what happens when power is applied. 


\section{CONCLUSION}

The current project presented many challenges, some were found early in the project and were overcome, and some presented themselves late in the project and will provide future students with opportunities to apply their problem solving skills. Overall, the experiment added a new chapter to Goldschmied's concept. This chapter made many changes from the previous tests in 1956, 1969, and 1982. The fan internally mounted in the aftbody of the model, the geometry upstream and downstream of the slot, the simplified data analysis process, and the propulsor related sensor suite are all improvements over the previous tests. The model differences make a direct comparison difficult for some parameters, but the external shape of the model and the function of the suction system remain the same. These similarities allow some comparisons to previous data. The closest possible comparison is between the surface pressure data taken in the 1956 tests and the NGP, even though the Reynolds numbers differ by a factor of four. The NGP surface pressure measurements did not show as much pressure recovery on the aftbody when suction was applied, and it showed a distinct negative offset when no suction was applied. The pressure data showed similar trends, but did not match up completely, and the differences cannot all be attributed to the difference in Reynolds number.

Independent of the comparisons to previous tests, the NGP was able to achieve some notable performance characteristics. It was able to achieve fully attached flow on the aftbody of the model with as little as $86 \mathrm{~W}$ of shaft power. It achieved a net positive axial force coefficient of 0.121 with a shaft power of only $172 \mathrm{~W}$ and with a slot gap of $0.7 \%$. It also achieved a peak axial force coefficient of 0.140 with a shaft power of $427 \mathrm{~W}$ and a slot gap of 1.6\%. "Pressure thrust" was achieved on the NGP with a peak axial pressure force coefficient of 0.005 with a shaft power of $427 \mathrm{~W}$ and a slot gap of $1.6 \%$ of the body length. A slightly lower axial pressure force coefficient of 0.0045 was achieved with only $172 \mathrm{~W}$ of shaft power and a slot gap of $0.7 \%$ of the body length. In fact, most slot gaps achieved their peak pressure force coefficient at a shaft power setting of $172 \mathrm{~W}$, or $200 \mathrm{~W}$ from the power supply.

Despite these achievements, an admittedly simple energy comparison between the NGP and a theoretical conventional propulsion system does not show a reduction in the required energy to achieve a desired axial force for the NGP configuration. In spite of this, the project is viewed as a success because a measurable and mostly repeatable performance was demonstrated, and the results of which were thoroughly documented and presented in a thorough and straightforward manner. 
The important surprise from the experiment is that the data showed insensitivity to the slot geometries tested. The cusp geometry that was tested did not show any measurable improvement over the baseline no-cusp data. This result was surprising as all available reports documented the slot geometry as a highly sensitive and important piece to the Goldschmied puzzle.

In general, the test results speak for themselves. The benefits presented in the test reports from the 1956, 1969, and 1982 tests of the Goldschmied Propulsor concept are not necessarily proven unachievable by the current round of testing. The only firm conclusion that this author is stating is that with the model and test equipment used in the current test, equivalently impressive performance was not achieved. This author believes that the Goldschmied Propulsor concept as a whole still holds much credibility and promise. It simply requires more study, analysis and testing to determine the conditions that are conducive to efficient operation. 


\section{RECOMMENDED FUTURE WORK}

Overall, the data presented here is a very good first step in refining the Goldschmied propulsor concept. Much more work should be completed to understand this concept more. The following are this author's opinions about what he sees as the best path forward for the Goldschmied Propulsor project at Cal Poly San Luis Obispo. Initially, there should be two parallel paths that eventually merge and culminate in a wind tunnel test of a much more ideal shape with a Goldschmied Propulsor. The first path is the refinement of the current model. In this first path, the issues that arose during the present testing are addressed and corrected and more testing is conducted. The second path involves analytical and numerical analysis conducted to define a body shape that would perform well at the Reynolds numbers that can be tested in the Cal Poly Aerospace Engineering Wind Tunnel.

\subsection{REFINEMENT OF THE NGP}

The first path entails further testing that should be completed with the following additions and redesigns of the NGP. The first areas needing improvement are the measurement of axial force, mass flow rate, and the sensor suite for the propulsor. These channels had higher than desired error, which should be reduced for future testing. Please contact the author for a more detailed explanation of these improvements.

Secondly, the flow field inside the tunnel needs to be further characterized. The current up-flow and sideflow is unknown. These two parameters need to be determined to make sure the model is aligned with the local flow direction. This was thought to be one possible source of the differences between the current testing and previous testing. Along with the up-flow and side-flow, the turbulence intensity and velocity uniformity should be measured and minimized through refinement of the tunnel inlet screen and honeycomb. All these parameters should be measured accurately and near the model mount location.

Third, remove select surface pressure taps from the axial pressure force measurement taps on the forward part of the forbody and repurpose them on the other sides of the model to examine the axisymmetric assumption of the flow around the model. Repurpose at least 12 taps, which would be three additional taps spaces out every $90^{\circ}$ radially and at the four additional lengthwise locations as key remaining taps on the right half. Alternatively, additional pressure sensors could be sourced and used for these additional ports.

Fourth, measure and characterize the electric motor efficiency, separately from the fan, for various operating conditions, including the motor temperature, load, and speed. This will be important to determine more accurately 
the shaft power of the fan. Prior to this, the fan motor cooling should be increased so that the motor can be run for longer periods without having to stop and cool it. This additional cooling was not done so far because it was unknown if these extra slits would severely hinder the fan performance. It is now believed that these will not hinder fan performance in any area of the test matrix that will matter.

Fifth, Design a test stand to mount the fan in freestream flow while running the tunnel. A simple way to do this would be to mount it to the sting balance. Do this to measure the fan thrust and torque. From this and the motor speed, calculate and efficiency in the freestream flow. Once the above is complete, compare the propulsive efficiency of the freestream fan to that with the fan installed in the model. This will be interesting for the BLI side of the concept.

Sixth, the calibration process for the axial force measurement, fan thrust, and the fan torque should be further developed to reduce the residuals. All pivoting mechanisms should be replaced with flexure joints to eliminate friction in the calibration setups. Modify the LabVIEW script to have a calibrate mode, where it calculates the calibration equation automatically, stores the coefficients, and uses them to process the data at the same time as it is measured.

Last, implement a way to bring the fan speed measurements into the LabVIEW data acquisition system with the other measurements. This will reduce time and hassle of having to post process and align this data.

\subsection{TEST MATRIX \& DATA AQUISITION SYSTEM}

The test matrix should be expanded to include more data points at lower fan power settings and slot gaps. These areas of the current test matrix are where the data is rapidly changing and shows promising performance. The exit area of the aftbody should be added as a controlled model parameter in the test matrix.

Also, continue the work with the LabVIEW VI traverse controller so the slot gap geometry can be changed automatically. The drivers for the VI are available, but due to time constraints, we were not able to complete this task and unfortunately resulted in having to change the slot gap manually.

\subsection{SHAPE DETERMINATION}

Alongside the above refined testing of the current NGP model, it is recommended that a new outer shape be developed that would likely perform better at the Reynolds number ranges that are achievable in the Cal Poly Aero 
Wind Tunnel. The goal of this work would be to not only provide a shape that could possibly show more efficient operation than a conventional propulsor, but it would establish a design process that could be used to create such a shape at any target Reynolds number. At the end of this path, the two paths would merge. The experimental lessons learned in the present and future NGP testing could be applied to building a model of this new shape with the goal to validate the design methodology by duplicating the expected performance from the design process. At the same time, the major benefits of the Goldschmied Propulsor concept would also be demonstrated.

One shape that has been suggested is a long cylindrical fuselage shape instead of the bluff body. The long cylinder would put the suction slot close to the maximum thickness. This could allow less negative axial pressure force for higher suction flow rates. The long cylindrical shape would also be representative of a typical modern cargo or transport aircraft. The model manufacturing for this type of shape would also be much simpler. The implementation of the Goldschmied Propulsor on such a shape would also allow more of a direct comparison to a typical aircraft design. It effectively would remove the shape of the aircraft as a source of difference and make the comparison more directly between the propulsors. 


\section{REFERENCES}

1 Drela, M., "Power Balance in Aerodynamic FLows," AIAA Journal, Vol. 47, No. 7, 2009, pp. 1761-1771.

2 Howe, H. J. and and Neumann, B. J. An Experimental Evaluation of a Low Propulsive Power, Discrete Suction Concept Applied to an Axisymmetric Vehicle; David W. Taylor Naval Ship R\&D Center, DTSNRDC TM-1682/02: Pg A-5 - A-7, January 1982.

3 Cerreta, P. A. Wind-Tunnel Investigation of the Drag of Proposed Boudnary-Layer-Controlled Airship; David Taylor Model Basin Aero Report 914, March 1957.

4 Goodyear Aircraft Corporation. An Investigation of a Boundary-Layer-Controlled Airship; Goodyear Aircraft Corporation GER-8399, October 1957.

5 Howe, H. J. and and Neumann, B. J. An Experimental Evaluation of a Low Propulsive Power, Discrete Suction Concept Aplied to an Axisymmetric Vehicle; David W. Taylor Naval Ship Research And Development Center DTNSRDC/TM-16-82/02, January 1982.

6 Goldschmied, F. R. A Theoretical Aerodynamic Analysis of a Boundary-Layer Controlled Airship Hull;

Goodyear Aircraft Corporation GER-6251: unavailable report, 1954.

7 Goldschmied, F. R. Aerodynamic Anaysis of the 1969 Wind-Tunnel Tests of the Goldschmied Body. Vol I -Slot Geometries and Body Pressure Distributions: Vol II - Boundary-Layer Suction, Transition, and Wake Drag; Westinghouse Electric Corp., R\&D Center, Research Report 77-1E9-BLCON: unavailable report, March 1977.

8 Goldschmied, F. R. Fuselage Self-Propulsion by Static-Pressure Thrust: Wind-Tunnel Verification; AIAA-872935, September 1987.

9 Ringleb, F. O. Separation Control by Trapped Vortices. In Boundary-Layer and Flow Controledited by G.V. Lachmann; Pergamon Press: New York, 1961; pp Vol. I, 265-294.

10 Goldschmied, F. R., "Aerodynamic Hull Design for HASPA LTA Optimization," Journal of Aircraft, Vol. 15, No. 9, 1978, pp. 634-638. 
11 Goldschmied, F. R. Wind Tunnel Tests of the Modified Goldschmied Model with Propulsion and Empennage: Analysis of Test Results; David W. Taylor Naval Ship R\&D Center DTNSRDC-ASED-CR-02-86 FRG-82-1, February 1986.

12 Braslow, A. L., Hicks, R. M., and Harris, R. V. J. Use of Grit-Type Boundary-Layer-Transition Trips on WInd Tunnel Models; NASA Technical Note TN D-3579, July 1965.

13 Altmann, G. F. An Inverstigative Study of Gurney Flaps on a NACA 0036 Airfoil; California Polytechnic State University, San Luis Obispo, March 2011.

14 Thomas, M. L., Pandey, D. V., and Nguyen, J. N. Flow Characteristics of the Renovated Cal Poly $3 \times 4 \mathrm{ft}$ Subsonic Wind Tunnel; California Polytechnic State University, San Luis Obispo, 2011.

15 Goldschmied, F. R., "Integrated Hull Design, Boundary-Layer COntrol, and Propulsion of Submerged Bodies," Journal of Hydronautics, Vol. 1, No. 1, June 1966, pp. 2-11.

16 ebm-papst Inc. http://www.ebmpapst.us. http://www.ebmpapst.us/allpdfs/4100N.PDF (accessed August 17, 2010).

17 Schubeler Composite. http://www.schuebeler-jets.de/en/. http://www.schuebeler-jets.de/en/hdt-fansen/measurements-hdt/doc_download/2-measurements-ds-30-dia-hdt-hacker-b40111.html (accessed September 20, 2010).

18 Hewlett Packard. Kurt's Manuals. http://128.238.9.201/ kurt/manuals/manuals/HP\%20Agilent/HP\%20601x\%20Series\%20User.pdf (accessed November 5, 2011).

19 Barlow, J. B., Rae, W. H., and Pope, A. Low Speed Wind Tunnel Testing, 3rd Edition, John Wiley \& Sons Inc., New York, 1999.

20 Thomason, N. Experimental Investigation of Suction Slot Geometry on a Goldschmied Propulsor; California Polytechnic State University, San Luis Obispo, February 2012. 
21 Maskel, E. C. A Theory of the Blockage Effects on Bluff Bodies and Stalled Wings in a Closed Wind Tunnel; Ministry of Aviation, Aeronautical Research Council Reports and Memoranda R\&M 3400, 1965.

22 Flachsbart, O. Recent Researches on the Air Resistance of Spheres; NACA Technical Memorandum TM-475 from Physikalische Zeitschrift Vol 28, 1927, 1928.

23 Raymer, D. P. Aircraft Design: A Conceptual Approach, American Institute of Aeronautics and Astronautics, Sylmar, 1992. 


\section{APPENDIX A - ERROR ANALYSIS}

Density

$$
\begin{gathered}
\rho=\frac{P_{\infty}}{R T_{\infty}} \\
\Delta \rho=\left\|\frac{\Delta P_{\infty}}{R T_{\infty}}, \frac{P_{\infty} \Delta T_{\infty}}{R\left(T_{\infty}\right)^{2}}\right\|_{2}
\end{gathered}
$$

Freestream dynamic Pressure

$$
\begin{gathered}
q_{\infty}=1.12\left(P_{t}-P_{s}\right) \\
\Delta q_{\infty}=1.12\left\|\Delta P_{t}, \Delta P_{s}\right\|_{2}
\end{gathered}
$$

Freestream Velocity

$$
\begin{gathered}
U_{\infty}=\left(\frac{2 q_{\infty}}{\rho}\right)^{\frac{1}{2}} \\
\Delta U_{\infty}=\left(\frac{\rho}{q_{\infty}}\right)^{\frac{1}{2}}\left\|\frac{\Delta q_{\infty}}{\rho}, \frac{q_{\infty} \Delta \rho}{\rho^{2}}\right\|_{2}
\end{gathered}
$$

Axial Force

$$
\begin{gathered}
F_{A, u}=123.41\left(v_{A}+\Delta V_{z s, A}\right)+3.4731 \\
\Delta F_{A, u}=\left\|123.41\left(\Delta v_{A}\right)\right\|
\end{gathered}
$$

Pressure Drag

$$
\begin{aligned}
C_{A, p} & =\sum_{i=1}^{N-1}-\left(\frac{C_{p, i+1}+C_{p, i}}{2}\right)\left[\frac{\pi\left(r_{i+1}^{2}-r_{i}^{2}\right)}{A_{r e f}}\right] \\
\Delta C_{A, p} & =\left\|\left(\frac{\Delta C_{p, i+1}+\Delta C_{p, i}}{2}\right)\left[\frac{\pi\left(r_{i+1}{ }^{2}-r_{i}^{2}\right)}{A_{\text {ref }}}\right]\right\|_{2}
\end{aligned}
$$

Pressure Coefficient 
Appendix A - Error Analysis

$$
\begin{gathered}
C_{p, i}=\frac{p-P_{\infty}}{q_{\infty}} \\
C_{p, i}=\frac{p-P_{\infty}}{q_{\infty}} \\
\Delta C p, i=\left\|\frac{\Delta p+\Delta P_{s}}{q_{\infty}}, \frac{p+P_{s}}{q_{\infty}^{2}} \Delta q_{\infty}\right\|_{2}
\end{gathered}
$$

Exit Speed

$$
\Delta U_{e}=\left(\frac{\rho}{q_{e}}\right)^{\frac{1}{2}}\left\|\frac{\Delta q_{e}}{\rho_{e}}, \frac{q_{e} \Delta \rho_{e}}{\rho_{e}{ }^{2}}\right\|_{2}
$$

Suction Mass Flow Rate

$$
\begin{gathered}
\dot{m}_{s}=\rho_{e} U_{e} A_{e} \\
\Delta \dot{m}_{s}=A_{e}\left\|\Delta \rho_{e} U_{e}, \rho_{e} \Delta U_{e}\right\|_{2}
\end{gathered}
$$

Fan Power

$$
\begin{gathered}
P_{S}=0.855 V_{p s} I_{p s} \\
\Delta P_{s}=0.855\left\|V_{p s} \Delta I_{p s}, \Delta V_{p s} I_{p s}\right\|
\end{gathered}
$$


APPENDIX B - PROCESSED DATA WITH ERROR ESTIMATES

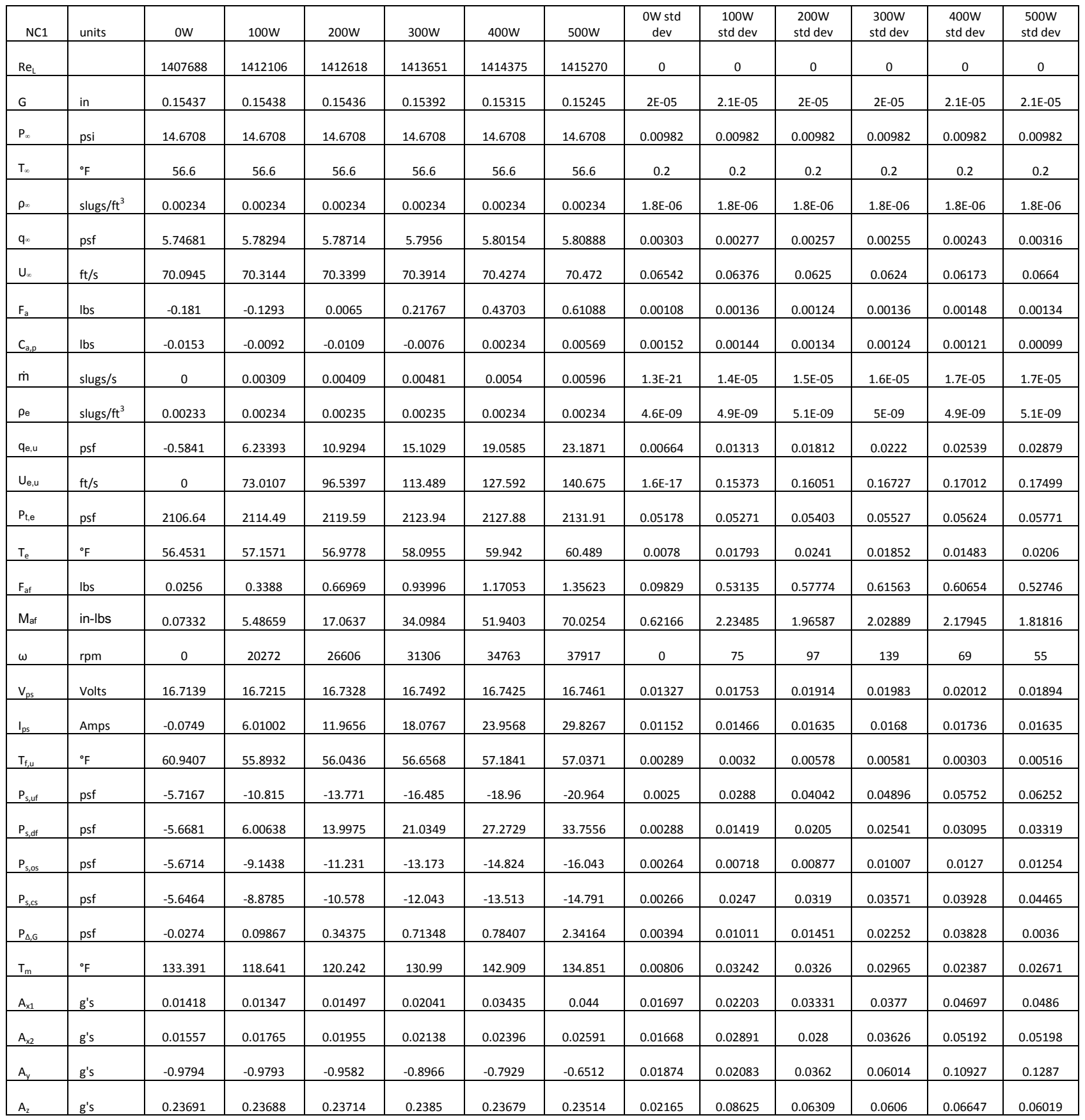




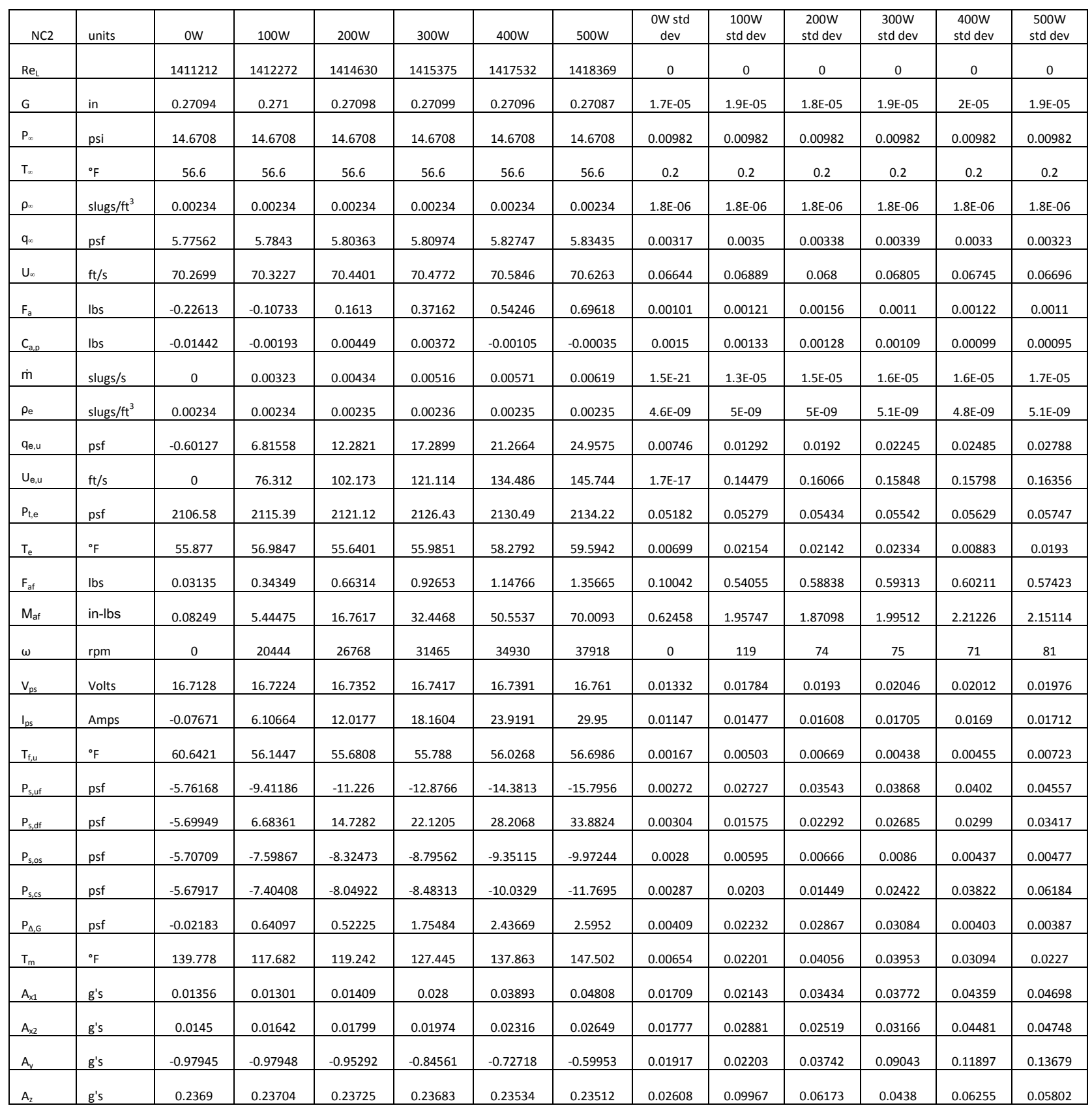




\begin{tabular}{|c|c|c|c|c|c|c|c|c|c|c|c|c|c|}
\hline NC3 & units & ow & $100 \mathrm{~W}$ & $200 W$ & $300 \mathrm{~W}$ & $400 \mathrm{~W}$ & $500 \mathrm{~W}$ & $\begin{array}{c}\text { OW std } \\
\text { dev }\end{array}$ & $\begin{array}{c}100 \mathrm{~W} \\
\text { std dev }\end{array}$ & $\begin{array}{c}200 W \\
\text { std dev }\end{array}$ & $\begin{array}{c}\text { 300W } \\
\text { std dev }\end{array}$ & $\begin{array}{c}400 \mathrm{~W} \\
\text { std dev }\end{array}$ & $\begin{array}{c}500 \mathrm{~W} \\
\text { std dev }\end{array}$ \\
\hline $\mathrm{Re}_{\mathrm{L}}$ & & 1405317 & 1408431 & 1411069 & 1413399 & 1413558 & 1414069 & 0 & 0 & 0 & 0 & 0 & 0 \\
\hline G & in & 0.38437 & 0.38437 & 0.38439 & 0.38438 & 0.3844 & 0.38439 & $1.7 \mathrm{E}-05$ & $1.8 \mathrm{E}-05$ & $1.8 \mathrm{E}-05$ & $1.8 \mathrm{E}-05$ & $1.9 \mathrm{E}-05$ & $1.9 \mathrm{E}-05$ \\
\hline$P_{\infty}$ & psi & 14.6708 & 14.6708 & 14.6708 & 14.6708 & 14.6708 & 14.6708 & 0.00982 & 0.00982 & 0.00982 & 0.00982 & 0.00982 & 0.00982 \\
\hline$T_{\infty}$ & ${ }^{\circ} \mathrm{F}$ & 56.6 & 56.6 & 56.6 & 56.6 & 56.6 & 56.6 & 0.2 & 0.2 & 0.2 & 0.2 & 0.2 & 0.2 \\
\hline$\rho_{\infty}$ & slugs $/ \mathrm{ft}^{3}$ & 0.00234 & 0.00234 & 0.00234 & 0.00234 & 0.00234 & 0.00234 & $1.8 \mathrm{E}-06$ & $1.8 \mathrm{E}-06$ & $1.8 \mathrm{E}-06$ & $1.8 \mathrm{E}-06$ & $1.8 \mathrm{E}-06$ & $1.8 \mathrm{E}-06$ \\
\hline$q_{\infty}$ & psf & 5.72747 & 5.75288 & 5.77445 & 5.79354 & 5.79484 & 5.79903 & 0.00267 & 0.00255 & 0.00249 & 0.00256 & 0.00276 & 0.00302 \\
\hline $\mathrm{U}_{\infty}$ & $\mathrm{ft} / \mathrm{s}$ & 69.9764 & 70.1314 & 70.2628 & 70.3788 & 70.3867 & 70.4122 & 0.06297 & 0.06233 & 0.06199 & 0.06246 & 0.0637 & 0.06543 \\
\hline $\mathrm{F}_{\mathrm{a}}$ & lbs & -0.35457 & -0.25976 & 0.01301 & 0.25069 & 0.41301 & 0.5926 & 0.00114 & 0.00158 & 0.00141 & 0.00108 & 0.00113 & 0.00101 \\
\hline$C_{a, p}$ & Ibs & -0.01364 & -0.0007 & $-4.6 \mathrm{E}-05$ & -0.00292 & -0.00165 & -0.00202 & 0.00151 & 0.00131 & 0.00119 & 0.00103 & 0.00099 & 0.00097 \\
\hline$\dot{\mathrm{m}}$ & slugs/s & 0 & 0.00328 & 0.0044 & 0.00534 & 0.00597 & 0.00647 & $1.5 \mathrm{E}-21$ & $1.4 \mathrm{E}-05$ & $1.6 \mathrm{E}-05$ & $1.5 \mathrm{E}-05$ & $1.6 \mathrm{E}-05$ & $1.8 \mathrm{E}-05$ \\
\hline$\rho_{\mathrm{e}}$ & slugs $/ \mathrm{ft}^{3}$ & 0.00233 & 0.00234 & 0.00235 & 0.00235 & 0.00235 & 0.00235 & 4.6E-09 & $4.9 \mathrm{E}-09$ & $4.9 \mathrm{E}-09$ & $6 \mathrm{E}-09$ & $4.9 \mathrm{E}-09$ & $5 \mathrm{E}-09$ \\
\hline $\mathrm{q}_{\mathrm{e}, \mathrm{u}}$ & psf & -0.60573 & 7.04138 & 12.6685 & 18.643 & 23.2989 & 27.3418 & 0.00756 & 0.01404 & 0.02049 & 0.02213 & 0.02548 & 0.03047 \\
\hline$U_{e, u}$ & $\mathrm{ft} / \mathrm{s}$ & 0 & 77.5962 & 103.922 & 126.068 & 140.953 & 152.606 & 1.7E-17 & 0.15474 & 0.16859 & 0.1501 & 0.15456 & 0.17068 \\
\hline $\mathrm{P}_{\mathrm{t}, \mathrm{e}}$ & psf & 2106.59 & 2115.7 & 2121.7 & 2128.01 & 2132.85 & 2137 & 0.05182 & 0.0531 & 0.05475 & 0.05548 & 0.05662 & 0.05872 \\
\hline $\mathrm{T}_{\mathrm{e}}$ & ${ }^{\circ} \mathrm{F}$ & 57.5947 & 57.4747 & 57.3517 & 58.9255 & 60.2675 & 60.6935 & 0.00737 & 0.01722 & 0.01818 & 0.04482 & 0.01479 & 0.0143 \\
\hline$F_{a f}$ & lbs & 0.03047 & 0.34129 & 0.64063 & 0.91886 & 1.15504 & 1.36896 & 0.10033 & 0.55428 & 0.59391 & 0.62972 & 0.60843 & 0.5847 \\
\hline $\mathrm{M}_{\mathrm{af}}$ & in-lbs & 0.07361 & 5.08879 & 16.1897 & 31.4282 & 48.9113 & 69.0054 & 0.62375 & 2.21989 & 2.24468 & 2.31608 & 2.46391 & 2.38306 \\
\hline$\omega$ & $\mathrm{rpm}$ & 0 & 20519 & 26734 & 31388 & 34879 & 37871 & 0 & 116 & 111 & 63 & 49 & 40 \\
\hline$V_{p s}$ & Volts & 16.7138 & 16.7228 & 16.729 & 16.733 & 16.7436 & 16.7655 & 0.01317 & 0.01718 & 0.01891 & 0.01995 & 0.01988 & 0.0195 \\
\hline $\mathrm{I}_{\mathrm{ps}}$ & Amps & -0.07467 & 6.08239 & 11.9803 & 18.0682 & 23.8882 & 29.9662 & 0.01119 & 0.01474 & 0.01566 & 0.01698 & 0.01655 & 0.01666 \\
\hline$T_{f, u}$ & ${ }^{\circ} \mathrm{F}$ & 61.7851 & 56.9741 & 56.3433 & 56.1343 & 56.3241 & 56.5544 & 0.00202 & 0.00522 & 0.00482 & 0.00347 & 0.0048 & 0.00365 \\
\hline$P_{s, \text { uf }}$ & psf & -5.75373 & -8.78776 & -9.97399 & -11.8524 & -13.4216 & -14.8528 & 0.00224 & 0.02681 & 0.0318 & 0.03135 & 0.03535 & 0.0394 \\
\hline$P_{s, d f}$ & psf & -5.68497 & 6.80262 & 14.8837 & 22.0987 & 28.4253 & 34.301 & 0.0025 & 0.01559 & 0.02261 & 0.02541 & 0.02892 & 0.03177 \\
\hline$P_{s, 0 s}$ & psf & -5.71451 & -6.9873 & -7.32402 & -7.26725 & -7.62654 & -7.98495 & 0.00211 & 0.00549 & 0.00678 & 0.00301 & 0.00312 & 0.00338 \\
\hline$P_{s, c s}$ & psf & -5.68897 & -6.94172 & -6.9145 & -6.95215 & -7.16794 & -7.4155 & 0.00215 & 0.02064 & 0.01166 & 0.00635 & 0.00713 & 0.00778 \\
\hline$P_{\Delta, G}$ & psf & -0.02072 & 0.58154 & 1.02076 & 1.99053 & 2.092 & 2.15461 & 0.00355 & 0.01955 & 0.02738 & 0.00836 & 0.00979 & 0.01097 \\
\hline$T_{m}$ & ${ }^{\circ} \mathrm{F}$ & 130.007 & 115.613 & 116.025 & 123.444 & 131.934 & 142.436 & 0.00725 & 0.02982 & 0.01864 & 0.04116 & 0.02143 & 0.02011 \\
\hline$A_{x 1}$ & g's & 0.01296 & 0.01227 & 0.01289 & 0.02473 & 0.04227 & 0.04944 & 0.01725 & 0.02478 & 0.03774 & 0.03561 & 0.04361 & 0.0451 \\
\hline$A_{x 2}$ & g's & 0.01355 & 0.01539 & 0.0173 & 0.01902 & 0.02141 & 0.02333 & 0.01735 & 0.02942 & 0.02521 & 0.02913 & 0.03844 & 0.0377 \\
\hline$A_{y}$ & g's & -0.97974 & -0.97966 & -0.95866 & -0.86824 & -0.71076 & -0.57782 & 0.0205 & 0.02161 & 0.03218 & 0.08301 & 0.12757 & 0.15082 \\
\hline $\mathrm{A}_{2}$ & g's & 0.2354 & 0.23707 & 0.23545 & 0.23696 & 0.23439 & 0.23451 & 0.01758 & 0.11165 & 0.06687 & 0.04596 & 0.05812 & 0.05365 \\
\hline
\end{tabular}




\begin{tabular}{|c|c|c|c|c|c|c|c|c|c|c|c|c|c|}
\hline NC4 & units & ow & $100 \mathrm{~W}$ & $200 W$ & $300 \mathrm{~W}$ & $400 \mathrm{~W}$ & $500 \mathrm{~W}$ & $\begin{array}{c}\text { OW std } \\
\text { dev }\end{array}$ & $\begin{array}{c}100 \mathrm{~W} \\
\text { std dev }\end{array}$ & $\begin{array}{c}200 W \\
\text { std dev }\end{array}$ & $\begin{array}{c}300 \mathrm{~W} \\
\text { std dev }\end{array}$ & $\begin{array}{c}400 \mathrm{~W} \\
\text { std dev }\end{array}$ & $\begin{array}{c}500 \mathrm{~W} \\
\text { std dev }\end{array}$ \\
\hline $\mathrm{Re}_{\mathrm{L}}$ & & 1406407 & 1408392 & 1410949 & 1411954 & 1414124 & 1415380 & 0 & 0 & 0 & 0 & 0 & 0 \\
\hline G & in & 0.50139 & 0.50135 & 0.50137 & 0.50137 & 0.50134 & 0.50135 & $1.5 \mathrm{E}-05$ & $1.7 \mathrm{E}-05$ & $1.8 \mathrm{E}-05$ & $1.8 \mathrm{E}-05$ & $1.8 \mathrm{E}-05$ & $1.8 \mathrm{E}-05$ \\
\hline$P_{\infty}$ & psi & 14.6708 & 14.6708 & 14.6708 & 14.6708 & 14.6708 & 14.6708 & 0.00982 & 0.00982 & 0.00982 & 0.00982 & 0.00982 & 0.00982 \\
\hline$T_{\infty}$ & ${ }^{\circ} \mathrm{F}$ & 56.6 & 56.6 & 56.6 & 56.6 & 56.6 & 56.6 & 0.2 & 0.2 & 0.2 & 0.2 & 0.2 & 0.2 \\
\hline$\rho_{\infty}$ & slugs $/ \mathrm{ft}^{3}$ & 0.00234 & 0.00234 & 0.00234 & 0.00234 & 0.00234 & 0.00234 & $1.8 \mathrm{E}-06$ & $1.8 \mathrm{E}-06$ & $1.8 \mathrm{E}-06$ & $1.8 \mathrm{E}-06$ & $1.8 \mathrm{E}-06$ & $1.8 \mathrm{E}-06$ \\
\hline$q_{\infty}$ & psf & 5.73636 & 5.75256 & 5.77346 & 5.78169 & 5.79948 & 5.80978 & 0.00342 & 0.0032 & 0.00301 & 0.00274 & 0.00267 & 0.00271 \\
\hline $\mathrm{U}_{\infty}$ & $\mathrm{ft} / \mathrm{s}$ & 70.0307 & 70.1295 & 70.2568 & 70.3069 & 70.4149 & 70.4774 & 0.06816 & 0.0666 & 0.06535 & 0.06359 & 0.06317 & 0.06345 \\
\hline $\mathrm{F}_{\mathrm{a}}$ & lbs & -0.84027 & -0.77127 & -0.51276 & -0.23618 & -0.07901 & 0.09958 & 0.00096 & 0.00144 & 0.00145 & 0.0011 & 0.00104 & 0.00113 \\
\hline$C_{a, p}$ & Ibs & -0.01546 & -0.00503 & 0.00051 & -0.00804 & -0.01101 & -0.01357 & 0.0015 & 0.00128 & 0.00122 & 0.00105 & 0.00102 & 0.001 \\
\hline$\dot{\mathrm{m}}$ & slugs/s & 0 & 0.00326 & 0.00449 & 0.00542 & 0.00607 & 0.00655 & $1.5 \mathrm{E}-21$ & $1.6 \mathrm{E}-05$ & 1.7E-05 & $1.6 \mathrm{E}-05$ & $1.7 \mathrm{E}-05$ & $1.8 \mathrm{E}-05$ \\
\hline$\rho_{\mathrm{e}}$ & slugs $/ \mathrm{ft}^{3}$ & 0.00233 & 0.00234 & 0.00235 & 0.00235 & 0.00235 & 0.00236 & 4.6E-09 & $4.8 \mathrm{E}-09$ & $5.1 \mathrm{E}-09$ & $5.2 \mathrm{E}-09$ & $5 E-09$ & 5.1E-09 \\
\hline $\mathrm{q}_{\mathrm{e}, \mathrm{u}}$ & psf & -0.61231 & 6.97664 & 13.1355 & 19.1328 & 23.975 & 27.8625 & 0.00776 & 0.01632 & 0.02283 & 0.02401 & 0.02842 & 0.03162 \\
\hline$U_{e, u}$ & $\mathrm{ft} / \mathrm{s}$ & 0 & 77.2645 & 105.786 & 127.655 & 142.815 & 153.803 & $1.8 \mathrm{E}-17$ & 0.18057 & 0.18451 & 0.16079 & 0.17011 & 0.17577 \\
\hline $\mathrm{P}_{\mathrm{t}, \mathrm{e}}$ & psf & 2106.55 & 2115.69 & 2122.33 & 2128.73 & 2133.78 & 2137.84 & 0.05178 & 0.05351 & 0.05572 & 0.05616 & 0.05786 & 0.05924 \\
\hline $\mathrm{T}_{\mathrm{e}}$ & ${ }^{\circ} \mathrm{F}$ & 57.8681 & 57.8264 & 57.1653 & 58.618 & 59.2498 & 59.1849 & 0.00636 & 0.01525 & 0.02257 & 0.02524 & 0.01421 & 0.01882 \\
\hline$F_{a f}$ & lbs & -0.06361 & 0.24075 & 0.55648 & 0.83986 & 1.06811 & 1.2645 & 0.09807 & 0.62628 & 0.67631 & 0.70213 & 0.70387 & 0.65778 \\
\hline $\mathrm{M}_{\mathrm{af}}$ & in-lbs & 0.60343 & 2.3188 & 10.6937 & 24.7174 & 40.2585 & 58.334 & 0.60613 & 2.56689 & 2.71669 & 2.98633 & 2.83694 & 2.69443 \\
\hline$\omega$ & $\mathrm{rpm}$ & 0 & 20335 & 26811 & 31393 & 34755 & 37645 & 0 & 185 & 122 & 98 & 71 & 120 \\
\hline$V_{p s}$ & Volts & 16.7118 & 16.7245 & 16.7291 & 16.7395 & 16.7448 & 16.7517 & 0.01284 & 0.01725 & 0.01884 & 0.01951 & 0.01903 & 0.01939 \\
\hline $\mathrm{I}_{\mathrm{ps}}$ & Amps & -0.07357 & 6.00769 & 11.9936 & 18.1787 & 23.8829 & 29.6793 & 0.01075 & 0.0143 & 0.01574 & 0.0166 & 0.01627 & 0.01615 \\
\hline$T_{f, u}$ & ${ }^{\circ} \mathrm{F}$ & 62.6762 & 57.3004 & 56.6105 & 56.3613 & 56.5279 & 56.4457 & 0.00223 & 0.0058 & 0.0058 & 0.00537 & 0.00298 & 0.00469 \\
\hline$P_{s, \text { uf }}$ & psf & -5.75318 & -8.43474 & -9.83523 & -11.891 & -13.6239 & -15.1145 & 0.00301 & 0.02718 & 0.03075 & 0.03117 & 0.03425 & 0.03716 \\
\hline$P_{s, d f}$ & psf & -5.68059 & 6.70096 & 14.9879 & 22.001 & 28.1996 & 33.768 & 0.00326 & 0.01784 & 0.02227 & 0.02439 & 0.02806 & 0.03144 \\
\hline$P_{s, 0 s}$ & psf & -5.71611 & -6.5183 & -6.78779 & -6.67341 & -6.88564 & -7.09071 & 0.00302 & 0.00599 & 0.00789 & 0.00302 & 0.00276 & 0.00275 \\
\hline$P_{s, c s}$ & psf & -5.69003 & -6.52619 & -6.60795 & -6.70275 & -6.81821 & -7.00254 & 0.00308 & 0.00947 & 0.01112 & 0.01679 & 0.0157 & 0.01273 \\
\hline$P_{\Delta, G}$ & psf & -0.03174 & 0.70943 & 0.79107 & 1.35087 & 1.24553 & 1.17425 & 0.005 & 0.01958 & 0.02381 & 0.01119 & 0.01245 & 0.01351 \\
\hline$T_{m}$ & ${ }^{\circ} \mathrm{F}$ & 131.349 & 112.234 & 111.717 & 121.144 & 132.145 & 142.394 & 0.0103 & 0.01883 & 0.03681 & 0.02986 & 0.02933 & 0.02458 \\
\hline$A_{x 1}$ & g's & 0.01184 & 0.01098 & 0.01332 & 0.02599 & 0.04025 & 0.04837 & 0.01647 & 0.02193 & 0.03895 & 0.0366 & 0.0424 & 0.04446 \\
\hline$A_{x 2}$ & g's & 0.01282 & 0.01468 & 0.01675 & 0.01833 & 0.02025 & 0.02254 & 0.01634 & 0.02614 & 0.02576 & 0.02894 & 0.03515 & 0.03595 \\
\hline$A_{y}$ & g's & -0.9796 & -0.97966 & -0.95294 & -0.84886 & -0.69864 & -0.58068 & 0.01756 & 0.02099 & 0.04169 & 0.1039 & 0.15118 & 0.17755 \\
\hline $\mathrm{A}_{2}$ & g's & 0.23635 & 0.23521 & 0.23586 & 0.23617 & 0.2348 & 0.23519 & 0.0229 & 0.09694 & 0.05636 & 0.0467 & 0.05728 & 0.0536 \\
\hline
\end{tabular}




\begin{tabular}{|c|c|c|c|c|c|c|c|c|c|c|c|c|c|}
\hline NC5 & units & ow & $100 \mathrm{~W}$ & $200 W$ & $300 \mathrm{~W}$ & $400 \mathrm{~W}$ & $500 \mathrm{~W}$ & $\begin{array}{c}\text { OW std } \\
\text { dev }\end{array}$ & $\begin{array}{c}100 \mathrm{~W} \\
\text { std dev }\end{array}$ & $\begin{array}{c}200 \mathrm{~W} \\
\text { std dev }\end{array}$ & $\begin{array}{c}300 \mathrm{~W} \\
\text { std dev }\end{array}$ & $\begin{array}{c}400 \mathrm{~W} \\
\text { std dev }\end{array}$ & $\begin{array}{c}500 \mathrm{~W} \\
\text { std dev }\end{array}$ \\
\hline $\mathrm{Re}_{\mathrm{L}}$ & & 1409776 & 1410278 & 1413603 & 1412656 & 1413865 & 1415487 & 0 & 0 & 0 & 0 & 0 & 0 \\
\hline G & in & 0.61753 & 0.61751 & 0.6175 & 0.6175 & 0.61748 & 0.61747 & 1.7E-05 & $1.8 \mathrm{E}-05$ & $1.9 \mathrm{E}-05$ & $1.9 \mathrm{E}-05$ & $2 \mathrm{E}-05$ & $1.9 \mathrm{E}-05$ \\
\hline$P_{\infty}$ & psi & 14.6708 & 14.6708 & 14.6708 & 14.6708 & 14.6708 & 14.6708 & 0.00982 & 0.00982 & 0.00982 & 0.00982 & 0.00982 & 0.00982 \\
\hline$T_{\infty}$ & ${ }^{\circ} \mathrm{F}$ & 56.6 & 56.6 & 56.6 & 56.6 & 56.6 & 56.6 & 0.2 & 0.2 & 0.2 & 0.2 & 0.2 & 0.2 \\
\hline$\rho_{\infty}$ & slugs $/ \mathrm{ft}^{3}$ & 0.00234 & 0.00234 & 0.00234 & 0.00234 & 0.00234 & 0.00234 & $1.8 \mathrm{E}-06$ & $1.8 \mathrm{E}-06$ & $1.8 \mathrm{E}-06$ & $1.8 \mathrm{E}-06$ & $1.8 \mathrm{E}-06$ & $1.8 \mathrm{E}-06$ \\
\hline $\mathrm{q}_{\infty}$ & psf & 5.76387 & 5.76797 & 5.79521 & 5.78744 & 5.79736 & 5.81066 & 0.00254 & 0.00297 & 0.00326 & 0.00319 & 0.00342 & 0.00329 \\
\hline $\mathrm{U}_{\infty}$ & $\mathrm{ft} / \mathrm{s}$ & 70.1984 & 70.2234 & 70.389 & 70.3418 & 70.402 & 70.4828 & 0.0623 & 0.06507 & 0.06713 & 0.06659 & 0.06831 & 0.06738 \\
\hline $\mathrm{F}_{\mathrm{a}}$ & lbs & -0.29872 & -0.1299 & 0.18669 & 0.39022 & 0.5874 & 0.80467 & 0.00087 & 0.0032 & 0.00162 & 0.00107 & 0.00107 & 0.0011 \\
\hline$C_{a, p}$ & lbs & -0.01773 & -0.01625 & -0.00196 & -0.01349 & -0.01864 & -0.02019 & 0.00149 & 0.00112 & 0.00115 & 0.00108 & 0.00105 & 0.00104 \\
\hline m் & slugs/s & 0 & 0.00323 & 0.00453 & 0.00547 & 0.00615 & 0.00667 & 1.4E-21 & $1.5 \mathrm{E}-05$ & $1.6 \mathrm{E}-05$ & 1.7E-05 & $1.8 \mathrm{E}-05$ & $1.8 \mathrm{E}-05$ \\
\hline$\rho_{\mathrm{e}}$ & slugs $/ \mathrm{ft}^{3}$ & 0.00233 & 0.00233 & 0.00234 & 0.00234 & 0.00235 & 0.00235 & 4.6E-09 & 4.7E-09 & $5 \mathrm{E}-09$ & 5.1E-09 & $5 \mathrm{E}-09$ & 5.3E-09 \\
\hline $\mathrm{q}_{\mathrm{e}, \mathrm{u}}$ & psf & -0.65163 & 6.87733 & 13.4423 & 19.5833 & 24.7399 & 28.9661 & 0.00772 & 0.01537 & 0.02119 & 0.02617 & 0.03021 & 0.033 \\
\hline $\mathrm{U}_{\mathrm{e}, \mathrm{u}}$ & $\mathrm{ft} / \mathrm{s}$ & 0 & 76.8246 & 107.163 & 129.267 & 145.24 & 156.944 & 1.7E-17 & 0.17103 & 0.16905 & 0.17308 & 0.17781 & 0.17977 \\
\hline $\mathrm{P}_{\mathrm{t}, \mathrm{e}}$ & psf & 2106.49 & 2115.3 & 2122.57 & 2128.97 & 2134.31 & 2138.66 & 0.0518 & 0.05347 & 0.05519 & 0.057 & 0.05865 & 0.0598 \\
\hline $\mathrm{T}_{\mathrm{e}}$ & ${ }^{\circ} \mathrm{F}$ & 57.1221 & 59.27 & 58.6888 & 59.6434 & 60.5847 & 60.2271 & 0.00626 & 0.01153 & 0.01856 & 0.02022 & 0.01499 & 0.02566 \\
\hline$F_{a f}$ & lbs & -0.02104 & 0.28171 & 0.60396 & 0.88495 & 1.12599 & 1.3398 & 0.09671 & 0.69871 & 0.75251 & 0.78374 & 0.775 & 0.73742 \\
\hline $\mathrm{M}_{\mathrm{af}}$ & in-lbs & 0.0676 & 5.11892 & 14.7324 & 30.6825 & 48.9897 & 73.3835 & 0.60748 & 3.25573 & 3.49023 & 3.845 & 3.7854 & 3.49122 \\
\hline$\omega$ & $\mathrm{rpm}$ & 0 & 20136 & 26774 & 31307 & 34810 & 37628 & 0 & 130 & 56 & 108 & 89 & 75 \\
\hline$V_{p s}$ & Volts & 16.7134 & 16.72 & 16.7286 & 16.7428 & 16.7514 & 16.7595 & 0.01209 & 0.01688 & 0.01877 & 0.01947 & 0.01928 & 0.01829 \\
\hline$I_{p s}$ & Amps & -0.07596 & 5.97607 & 11.9468 & 17.9984 & 23.973 & 29.827 & 0.0102 & 0.01455 & 0.01553 & 0.01603 & 0.0164 & 0.01667 \\
\hline$T_{f, u}$ & ${ }^{\circ} \mathrm{F}$ & 58.134 & 58.1879 & 56.6512 & 56.7138 & 57.023 & 57.4178 & 0.00183 & 0.00447 & 0.00364 & 0.0039 & 0.00584 & 0.00265 \\
\hline$P_{s, \text { uf }}$ & psf & -5.78279 & -8.6681 & -9.77113 & -11.9271 & -13.8197 & -15.2896 & 0.00214 & 0.02237 & 0.02682 & 0.03046 & 0.03378 & 0.03775 \\
\hline$P_{s, d f}$ & psf & -5.70377 & 6.05201 & 14.956 & 22.0878 & 28.5626 & 34.1238 & 0.00252 & 0.01573 & 0.02121 & 0.02444 & 0.02858 & 0.03155 \\
\hline$P_{s, o s}$ & psf & -5.74609 & -6.25183 & -6.35579 & -6.39578 & -6.54249 & -6.70433 & 0.00211 & 0.00354 & 0.008 & 0.00461 & 0.00335 & 0.00328 \\
\hline$P_{s, c s}$ & psf & -5.71579 & -6.38278 & -6.42127 & -6.44381 & -6.66295 & -6.85637 & 0.0022 & 0.00541 & 0.01384 & 0.01006 & 0.01286 & 0.01582 \\
\hline$P_{\Delta, G}$ & psf & -0.00842 & 1.18734 & 1.02493 & 1.20878 & 1.25909 & 1.23706 & 0.00437 & 0.0059 & 0.01734 & 0.01116 & 0.00985 & 0.01085 \\
\hline$T_{m}$ & ${ }^{\circ} \mathrm{F}$ & 65.5467 & 64.0986 & 90.0934 & 104.363 & 122.927 & 142.126 & 0.0095 & 0.02481 & 0.02254 & 0.01286 & 0.01832 & 0.01712 \\
\hline$A_{x 1}$ & g's & 0.01088 & 0.00997 & 0.0123 & 0.02325 & 0.0406 & 0.04716 & 0.01599 & 0.02636 & 0.03986 & 0.03455 & 0.04368 & 0.04303 \\
\hline$A_{\times 2}$ & g's & 0.01186 & 0.01369 & 0.01546 & 0.01741 & 0.01946 & 0.0215 & 0.01672 & 0.02254 & 0.02395 & 0.02596 & 0.03535 & 0.03547 \\
\hline$A_{\gamma}$ & g's & -0.98009 & -0.97975 & -0.95885 & -0.86924 & -0.72265 & -0.58917 & 0.01874 & 0.02158 & 0.03247 & 0.07758 & 0.12621 & 0.13666 \\
\hline $\mathrm{A}_{2}$ & g's & 0.23453 & 0.23388 & 0.23651 & 0.2362 & 0.2344 & 0.23385 & 0.02614 & 0.08888 & 0.05431 & 0.04147 & 0.05643 & 0.05346 \\
\hline
\end{tabular}




\begin{tabular}{|c|c|c|c|c|c|c|c|c|c|c|c|c|c|}
\hline $\mathrm{C} 2$ & units & ow & $100 \mathrm{~W}$ & $200 W$ & $300 \mathrm{~W}$ & $400 \mathrm{~W}$ & $500 \mathrm{~W}$ & $\begin{array}{c}\text { OW std } \\
\text { dev }\end{array}$ & $\begin{array}{c}100 \mathrm{~W} \\
\text { std dev }\end{array}$ & $\begin{array}{c}200 \mathrm{~W} \\
\text { std dev }\end{array}$ & $\begin{array}{c}300 \mathrm{~W} \\
\text { std dev }\end{array}$ & $\begin{array}{c}400 \mathrm{~W} \\
\text { std dev }\end{array}$ & $\begin{array}{c}500 \mathrm{~W} \\
\text { std dev }\end{array}$ \\
\hline $\operatorname{Re}_{\mathrm{L}}$ & & 1412550 & 1415145 & 1415120 & 1417418 & 1419364 & 1418749 & 0 & 0 & 0 & 0 & 0 & 0 \\
\hline G & in & 0.45817 & 0.45816 & 0.45817 & 0.45816 & 0.45815 & 0.45814 & $1.6 \mathrm{E}-05$ & 1.7E-05 & $1.8 \mathrm{E}-05$ & 1.7E-05 & $1.9 \mathrm{E}-05$ & $1.8 \mathrm{E}-05$ \\
\hline$P_{\infty}$ & psi & 14.7248 & 14.7248 & 14.7248 & 14.7248 & 14.7248 & 14.7248 & 0.00982 & 0.00982 & 0.00982 & 0.00982 & 0.00982 & 0.00982 \\
\hline$T_{\infty}$ & ${ }^{\circ} \mathrm{F}$ & 56.2 & 56.2 & 56.2 & 56.2 & 56.2 & 56.2 & 0.2 & 0.2 & 0.2 & 0.2 & 0.2 & 0.2 \\
\hline$\rho_{\infty}$ & slugs/ $/ \mathrm{ft}^{3}$ & 0.00235 & 0.00235 & 0.00235 & 0.00235 & 0.00235 & 0.00235 & $1.8 \mathrm{E}-06$ & $1.8 \mathrm{E}-06$ & $1.8 \mathrm{E}-06$ & $1.8 \mathrm{E}-06$ & $1.8 \mathrm{E}-06$ & $1.8 \mathrm{E}-06$ \\
\hline$q_{\infty}$ & psf & 5.76096 & 5.78215 & 5.78195 & 5.80074 & 5.81668 & 5.81164 & 0.00253 & 0.00268 & 0.00286 & 0.00305 & 0.00307 & 0.00322 \\
\hline $\mathrm{U}_{\infty}$ & $\mathrm{ft} / \mathrm{s}$ & 70.0252 & 70.1539 & 70.1526 & 70.2665 & 70.363 & 70.3325 & 0.06197 & 0.06294 & 0.06409 & 0.0654 & 0.06554 & 0.06661 \\
\hline $\mathrm{F}_{\mathrm{a}}$ & lbs & -0.15371 & -0.03696 & 0.17535 & 0.44719 & 0.62042 & 0.79667 & 0.00118 & 0.00196 & 0.00398 & 0.00189 & 0.00157 & 0.00139 \\
\hline$C_{a, p}$ & lbs & -0.01034 & -0.00322 & 0.00544 & 0.01084 & 0.00486 & 0.00348 & 0.00151 & 0.00139 & 0.00127 & 0.00115 & 0.00102 & 0.00099 \\
\hline m் & slugs/s & 0 & 0.00312 & 0.0043 & 0.00513 & 0.00576 & 0.00624 & 1.4E-21 & $1.4 \mathrm{E}-05$ & $1.5 \mathrm{E}-05$ & 1.7E-05 & $1.7 \mathrm{E}-05$ & 1.7E-05 \\
\hline$\rho_{\mathrm{e}}$ & slugs $/ \mathrm{ft}^{3}$ & 0.00234 & 0.00235 & 0.00235 & 0.00235 & 0.00235 & 0.00235 & 4.7E-09 & 4.9E-09 & $4.9 \mathrm{E}-09$ & $4.9 \mathrm{E}-09$ & $4.8 \mathrm{E}-09$ & $5.1 \mathrm{E}-09$ \\
\hline $\mathrm{q}_{\mathrm{e}, \mathrm{u}}$ & psf & -0.46437 & 6.36789 & 12.0532 & 17.143 & 21.6016 & 25.3246 & 0.0063 & 0.01341 & 0.01891 & 0.02467 & 0.02631 & 0.02948 \\
\hline $\mathrm{U}_{\mathrm{e}, \mathrm{u}}$ & $\mathrm{ft} / \mathrm{s}$ & 0 & 73.6682 & 101.289 & 120.796 & 135.527 & 146.677 & 1.7E-17 & 0.15489 & 0.15892 & 0.17385 & 0.16523 & 0.17105 \\
\hline $\mathrm{P}_{\mathrm{t}, \mathrm{e}}$ & psf & 2114.38 & 2123 & 2129.18 & 2134.78 & 2139.71 & 2143.79 & 0.05177 & 0.05296 & 0.05413 & 0.05607 & 0.0569 & 0.05823 \\
\hline $\mathrm{T}_{\mathrm{e}}$ & ${ }^{\circ} \mathrm{F}$ & 56.2188 & 57.52 & 58.395 & 59.783 & 60.4511 & 60.9855 & 0.00979 & 0.01892 & 0.01749 & 0.01351 & 0.00908 & 0.01935 \\
\hline$F_{\text {af }}$ & lbs & -0.02581 & 0.32418 & 0.61909 & 0.88999 & 1.10948 & 1.30133 & 0.14651 & 0.62577 & 0.68519 & 0.70887 & 0.69511 & 0.6461 \\
\hline $\mathrm{M}_{\mathrm{af}}$ & in-lbs & 0.06561 & 5.16002 & 16.5906 & 32.7617 & 49.9998 & 68.9635 & 0.91963 & 2.28731 & 2.58461 & 2.83116 & 2.72528 & 2.43393 \\
\hline$\omega$ & $\mathrm{rpm}$ & 0 & 20402 & 26856 & 31481 & 35012 & 37853 & 0 & 101 & 95 & 76 & 64 & 87 \\
\hline$V_{p s}$ & Volts & 16.7081 & 16.7168 & 16.7287 & 16.7422 & 16.7417 & 16.7522 & 0.01861 & 0.02236 & 0.02381 & 0.02408 & 0.02372 & 0.02362 \\
\hline$I_{p s}$ & Amps & -0.02389 & 5.98225 & 12.0113 & 18.0334 & 23.9127 & 29.8828 & 0.01799 & 0.01927 & 0.0202 & 0.02026 & 0.0199 & 0.01976 \\
\hline$T_{f, u}$ & ${ }^{\circ} \mathrm{F}$ & 56.5419 & 56.167 & 56.2193 & 56.6737 & 56.817 & 57.3855 & 0.00132 & 0.00529 & 0.00467 & 0.00413 & 0.00253 & 0.00354 \\
\hline$P_{s, \text { uf }}$ & psf & -5.78848 & -9.17188 & -11.0793 & -12.7482 & -14.3097 & -15.7771 & 0.00221 & 0.0281 & 0.03518 & 0.03733 & 0.03866 & 0.04139 \\
\hline$P_{s, d f}$ & psf & -5.72745 & 6.64412 & 14.9171 & 22.1252 & 28.4861 & 34.4212 & 0.00269 & 0.01625 & 0.02342 & 0.02733 & 0.03045 & 0.03334 \\
\hline$P_{s, o s}$ & psf & -5.74255 & -7.18922 & -7.88903 & -8.25949 & -8.69975 & -9.18394 & 0.00227 & 0.00758 & 0.00625 & 0.00749 & 0.0037 & 0.00415 \\
\hline$P_{s, c s}$ & psf & -5.72903 & -7.28505 & -7.81177 & -8.20561 & -8.65567 & -9.17062 & 0.00229 & 0.00914 & 0.00865 & 0.00983 & 0.01246 & 0.02151 \\
\hline$P_{\Delta, G}$ & psf & -0.01346 & 0.32491 & 0.70538 & 1.38711 & 2.0513 & 2.10663 & 0.00389 & 0.01696 & 0.02528 & 0.03091 & 0.00581 & 0.00794 \\
\hline$T_{m}$ & ${ }^{\circ} \mathrm{F}$ & 66.9087 & 62.8827 & 78.2423 & 94.3303 & 110.521 & 125.529 & 0.00964 & 0.02629 & 0.0298 & 0.01843 & 0.01917 & 0.02506 \\
\hline$A_{x 1}$ & g's & 0.01104 & 0.01098 & 0.01163 & 0.024 & 0.04223 & 0.04997 & 0.01718 & 0.02239 & 0.03239 & 0.03741 & 0.04344 & 0.04629 \\
\hline$A_{\times 2}$ & g's & 0.01001 & 0.01193 & 0.01364 & 0.01472 & 0.01694 & 0.01968 & 0.01733 & 0.0269 & 0.02726 & 0.03002 & 0.03216 & 0.03579 \\
\hline$A_{\gamma}$ & g's & -0.97954 & -0.97965 & -0.95672 & -0.86082 & -0.70932 & -0.50216 & 0.01939 & 0.0214 & 0.03519 & 0.08647 & 0.13174 & 0.12963 \\
\hline $\mathrm{A}_{2}$ & g's & 0.24076 & 0.24113 & 0.24105 & 0.24182 & 0.2399 & 0.24032 & 0.02144 & 0.10238 & 0.04598 & 0.04742 & 0.05508 & 0.05371 \\
\hline
\end{tabular}




\begin{tabular}{|c|c|c|c|c|c|c|c|c|c|c|c|c|c|}
\hline $\mathrm{C} 3$ & units & ow & $100 \mathrm{~W}$ & $200 W$ & $300 \mathrm{~W}$ & $400 W$ & $500 \mathrm{~W}$ & $\begin{array}{c}\text { OW std } \\
\text { dev }\end{array}$ & $\begin{array}{c}100 \mathrm{~W} \\
\text { std dev }\end{array}$ & $\begin{array}{c}200 W \\
\text { std dev }\end{array}$ & $\begin{array}{c}\text { 300W } \\
\text { std dev }\end{array}$ & $\begin{array}{c}400 W \\
\text { std dev }\end{array}$ & $\begin{array}{c}500 \mathrm{~W} \\
\text { std dev }\end{array}$ \\
\hline $\mathrm{Re}_{\mathrm{L}}$ & & 1414199 & 1415893 & 1420279 & 1418093 & 1420797 & 1422611 & 0 & 0 & 0 & 0 & 0 & 0 \\
\hline G & in & 0.57347 & 0.57347 & 0.57349 & 0.57349 & 0.57348 & 0.57348 & 1.7E-05 & $1.9 \mathrm{E}-05$ & $1.9 \mathrm{E}-05$ & $2 \mathrm{E}-05$ & $2 \mathrm{E}-05$ & $2 \mathrm{E}-05$ \\
\hline$P_{\infty}$ & psi & 14.7248 & 14.7248 & 14.7248 & 14.7248 & 14.7248 & 14.7248 & 0.00982 & 0.00982 & 0.00982 & 0.00982 & 0.00982 & 0.00982 \\
\hline $\mathrm{T}_{\infty}$ & ${ }^{\circ} \mathrm{F}$ & 56.2 & 56.2 & 56.2 & 56.2 & 56.2 & 56.2 & 0.2 & 0.2 & 0.2 & 0.2 & 0.2 & 0.2 \\
\hline$\rho_{\infty}$ & slugs $/ \mathrm{ft}^{3}$ & 0.00235 & 0.00235 & 0.00235 & 0.00235 & 0.00235 & 0.00235 & $1.8 \mathrm{E}-06$ & $1.8 \mathrm{E}-06$ & $1.8 \mathrm{E}-06$ & $1.8 \mathrm{E}-06$ & $1.8 \mathrm{E}-06$ & $1.8 \mathrm{E}-06$ \\
\hline$q_{\infty}$ & psf & 5.77442 & 5.78826 & 5.82418 & 5.80627 & 5.82843 & 5.84332 & 0.00325 & 0.00319 & 0.00335 & 0.00334 & 0.00343 & 0.00334 \\
\hline $\mathrm{U}_{\infty}$ & $\mathrm{ft} / \mathrm{s}$ & 70.1069 & 70.1909 & 70.4084 & 70.3 & 70.434 & 70.524 & 0.06672 & 0.06636 & 0.06757 & 0.06747 & 0.06813 & 0.06754 \\
\hline $\mathrm{F}_{\mathrm{a}}$ & Ibs & -0.32118 & -0.18633 & 0.09697 & 0.35014 & 0.53706 & 0.73512 & 0.00135 & 0.00158 & 0.00259 & 0.0015 & 0.0017 & 0.00143 \\
\hline$C_{a, p}$ & lbs & -0.01088 & -0.00321 & 0.00376 & 0.00065 & -0.00308 & -0.0043 & 0.00163 & 0.00121 & 0.00122 & 0.00108 & 0.00105 & 0.00103 \\
\hline$\dot{\mathrm{m}}$ & slugs $/ \mathrm{s}$ & 0 & 0.00317 & 0.00441 & 0.00532 & 0.00597 & 0.00646 & $1.6 \mathrm{E}-21$ & $1.6 \mathrm{E}-05$ & $1.6 \mathrm{E}-05$ & $1.5 \mathrm{E}-05$ & $1.6 \mathrm{E}-05$ & 1.7E-05 \\
\hline$\rho_{\mathrm{e}}$ & slugs $/ \mathrm{ft}^{3}$ & 0.00234 & 0.00234 & 0.00235 & 0.00235 & 0.00235 & 0.00235 & $5.3 \mathrm{E}-09$ & 4.7E-09 & $5 \mathrm{E}-09$ & 4.9E-09 & 4.9E-09 & $5 \mathrm{E}-09$ \\
\hline $\mathrm{q}_{\mathrm{e}, \mathrm{u}}$ & psf & -0.51259 & 6.57861 & 12.6604 & 18.4329 & 23.1632 & 27.1267 & 0.00745 & 0.01602 & 0.02135 & 0.02192 & 0.02573 & 0.02992 \\
\hline $\mathrm{U}_{\mathrm{e}, \mathrm{u}}$ & $\mathrm{ft} / \mathrm{s}$ & 0 & 74.9094 & 103.805 & 125.196 & 140.28 & 151.831 & $1.9 \mathrm{E}-17$ & 0.18206 & 0.17509 & 0.14902 & 0.15611 & 0.16775 \\
\hline $\mathrm{P}_{\mathrm{t}, \mathrm{e}}$ & psf & 2114.33 & 2123.07 & 2129.96 & 2136.38 & 2141.47 & 2145.66 & 0.05641 & 0.05347 & 0.05503 & 0.05534 & 0.05674 & 0.05845 \\
\hline $\mathrm{T}_{\mathrm{e}}$ & ${ }^{\circ} \mathrm{F}$ & 55.9546 & 57.9904 & 58.5443 & 59.6485 & 60.4277 & 61.6296 & 0.0095 & 0.00816 & 0.01853 & 0.01452 & 0.01141 & 0.01734 \\
\hline$F_{a f}$ & Ibs & 0.00964 & 0.32047 & 0.63736 & 0.88565 & 1.11457 & 1.28614 & 0.10468 & 0.71068 & 0.76528 & 0.79174 & 0.8048 & 0.7632 \\
\hline $\mathrm{M}_{\mathrm{af}}$ & in-lbs & 0.06617 & 4.94746 & 15.5829 & 30.2226 & 48.7151 & 64.4963 & 0.73146 & 3.26657 & 3.45185 & 3.70169 & 3.51684 & 3.09747 \\
\hline$\omega$ & $\mathrm{rpm}$ & 0 & 20206 & 26848 & 31356 & 34900 & 37691 & 0 & 82 & 88 & 87 & 68 & 89 \\
\hline$V_{p s}$ & Volts & 16.716 & 16.7197 & 16.7272 & 16.7464 & 16.7456 & 16.7548 & 0.01876 & 0.02212 & 0.02367 & 0.02387 & 0.02402 & 0.02393 \\
\hline$I_{p s}$ & Amps & -0.07254 & 6.01078 & 12.0467 & 18.0344 & 23.947 & 29.762 & 0.01586 & 0.01873 & 0.0198 & 0.02061 & 0.02003 & 0.02077 \\
\hline$T_{f, u}$ & ${ }^{\circ} \mathrm{F}$ & 58.9851 & 56.1046 & 56.153 & 56.4074 & 56.9399 & 57.2298 & 0.0021 & 0.00376 & 0.00399 & 0.00311 & 0.0035 & 0.00442 \\
\hline$P_{s, u f}$ & psf & -5.80251 & -8.87709 & -10.041 & -11.7717 & -13.4227 & -14.8553 & 0.00293 & 0.02536 & 0.03352 & 0.03202 & 0.03712 & 0.04036 \\
\hline$P_{s, d f}$ & psf & -5.72412 & 6.3236 & 15.0595 & 22.2521 & 28.6995 & 34.2057 & 0.00319 & 0.01656 & 0.02217 & 0.02566 & 0.02969 & 0.03195 \\
\hline$P_{s, 0 s}$ & psf & -5.74832 & -6.72167 & -7.10029 & -7.06506 & -7.36899 & -7.65293 & 0.00303 & 0.00601 & 0.00713 & 0.0041 & 0.00359 & 0.00365 \\
\hline$P_{s, c s}$ & psf & -5.71909 & -6.73378 & -6.87374 & -6.85361 & -7.07834 & -7.29652 & 0.003 & 0.00808 & 0.01024 & 0.00593 & 0.00604 & 0.00684 \\
\hline$P_{\Delta, G}$ & psf & -0.01236 & 1.08024 & 0.78511 & 1.3374 & 1.21974 & 1.17383 & 0.00479 & 0.01482 & 0.02229 & 0.01214 & 0.013 & 0.0143 \\
\hline $\mathrm{T}_{\mathrm{m}}$ & ${ }^{\circ} \mathrm{F}$ & 121.062 & 98.9893 & 102.01 & 111.723 & 127.048 & 138.03 & 0.00658 & 0.02758 & 0.02603 & 0.02791 & 0.01846 & 0.01539 \\
\hline$A_{x 1}$ & g's & 0.01 & 0.0098 & 0.0109 & 0.02425 & 0.03926 & 0.04924 & 0.01838 & 0.01991 & 0.02983 & 0.03731 & 0.04276 & 0.04327 \\
\hline$A_{x 2}$ & g's & 0.00864 & 0.01074 & 0.01268 & 0.01426 & 0.01652 & 0.01869 & 0.01834 & 0.02105 & 0.02573 & 0.02702 & 0.03117 & 0.03376 \\
\hline$A_{y}$ & g's & -0.97984 & -0.97982 & -0.95679 & -0.85374 & -0.72496 & -0.55715 & 0.01933 & 0.02176 & 0.03368 & 0.09497 & 0.12539 & 0.14568 \\
\hline $\mathrm{A}_{2}$ & g's & 0.241 & 0.24105 & 0.24072 & 0.24036 & 0.23941 & 0.23856 & 0.01867 & 0.07823 & 0.05129 & 0.04359 & 0.05396 & 0.05664 \\
\hline
\end{tabular}




\begin{tabular}{|c|c|c|c|c|c|c|c|c|c|c|c|c|c|}
\hline C4 & units & OW & $100 \mathrm{~W}$ & $200 \mathrm{~W}$ & $300 \mathrm{~W}$ & $400 \mathrm{~W}$ & $500 \mathrm{~W}$ & $\begin{array}{c}\text { OW std } \\
\text { dev }\end{array}$ & $\begin{array}{c}100 \mathrm{~W} \\
\text { std dev }\end{array}$ & $\begin{array}{c}200 \mathrm{~W} \\
\text { std dev }\end{array}$ & $\begin{array}{c}300 \mathrm{~W} \\
\text { std dev }\end{array}$ & $\begin{array}{c}400 \mathrm{~W} \\
\text { std dev }\end{array}$ & $\begin{array}{c}500 \mathrm{~W} \\
\text { std dev }\end{array}$ \\
\hline $\operatorname{Re}_{L}$ & & 1413076 & 1416456 & 1419425 & 1419793 & 1421121 & 1422452 & 0 & 0 & 0 & 0 & 0 & 0 \\
\hline G & in & 0.68837 & 0.68838 & 0.68837 & 0.68838 & 0.68838 & 0.68836 & $1.8 \mathrm{E}-05$ & $2 \mathrm{E}-05$ & $2.1 \mathrm{E}-05$ & $2 \mathrm{E}-05$ & $2 \mathrm{E}-05$ & $2.1 \mathrm{E}-05$ \\
\hline$P_{\infty}$ & psi & 14.7248 & 14.7248 & 14.7248 & 14.7248 & 14.7248 & 14.7248 & 0.00982 & 0.00982 & 0.00982 & 0.00982 & 0.00982 & 0.00982 \\
\hline$T_{\infty}$ & ${ }^{\circ} \mathrm{F}$ & 56.2 & 56.2 & 56.2 & 56.2 & 56.2 & 56.2 & 0.2 & 0.2 & 0.2 & 0.2 & 0.2 & 0.2 \\
\hline$\rho_{\infty}$ & slugs $/ \mathrm{ft}^{3}$ & 0.00235 & 0.00235 & 0.00235 & 0.00235 & 0.00235 & 0.00235 & $1.8 \mathrm{E}-06$ & $1.8 \mathrm{E}-06$ & $1.8 \mathrm{E}-06$ & $1.8 \mathrm{E}-06$ & $1.8 \mathrm{E}-06$ & $1.8 \mathrm{E}-06$ \\
\hline$q_{\infty}$ & psf & 5.76525 & 5.79287 & 5.81718 & 5.82019 & 5.83109 & 5.84202 & 0.00339 & 0.00348 & 0.00339 & 0.00343 & 0.00333 & 0.00322 \\
\hline $\mathrm{U}_{\infty}$ & $\mathrm{ft} / \mathrm{s}$ & 70.0513 & 70.2188 & 70.366 & 70.3843 & 70.4501 & 70.5161 & 0.06776 & 0.06848 & 0.06784 & 0.0681 & 0.06745 & 0.06666 \\
\hline $\mathrm{F}_{\mathrm{a}}$ & lbs & -0.10427 & -0.03637 & 0.17932 & 0.40658 & 0.61599 & 0.78245 & 0.00122 & 0.00193 & 0.0036 & 0.00139 & 0.00142 & 0.00162 \\
\hline$C_{a, p}$ & lbs & -0.00977 & 0.00462 & 0.00795 & -0.00466 & -0.00863 & -0.0104 & 0.00149 & 0.00137 & 0.00123 & 0.00111 & 0.00108 & 0.00107 \\
\hline$\dot{\mathrm{m}}$ & slugs/s & 0 & 0.00321 & 0.00446 & 0.00536 & 0.00602 & 0.00652 & $1.6 \mathrm{E}-21$ & $1.4 \mathrm{E}-05$ & $1.6 \mathrm{E}-05$ & $1.5 \mathrm{E}-05$ & $1.6 \mathrm{E}-05$ & $1.7 \mathrm{E}-05$ \\
\hline$\rho_{\mathrm{e}}$ & slugs $/ \mathrm{ft}^{3}$ & 0.00234 & 0.00234 & 0.00235 & 0.00235 & 0.00235 & 0.00236 & 4.7E-09 & $4.8 \mathrm{E}-09$ & $4.9 \mathrm{E}-09$ & $4.8 \mathrm{E}-09$ & $4.9 \mathrm{E}-09$ & $5.2 \mathrm{E}-09$ \\
\hline $\mathrm{q}_{\mathrm{e}, \mathrm{u}}$ & psf & -0.56525 & 6.74505 & 12.9405 & 18.7225 & 23.606 & 27.6502 & 0.0078 & 0.01407 & 0.02117 & 0.02271 & 0.02595 & 0.02974 \\
\hline$U_{e, u}$ & $\mathrm{ft} / \mathrm{s}$ & 0 & 75.8907 & 104.951 & 126.126 & 141.606 & 153.202 & $1.9 \mathrm{E}-17$ & 0.15779 & 0.1717 & 0.15324 & 0.15597 & 0.16526 \\
\hline $\mathrm{P}_{\mathrm{t}, \mathrm{e}}$ & psf & 2114.3 & 2123.38 & 2130.22 & 2136.62 & 2141.87 & 2146.16 & 0.05179 & 0.05313 & 0.05499 & 0.05557 & 0.05677 & 0.05826 \\
\hline $\mathrm{T}_{\mathrm{e}}$ & ${ }^{\circ} \mathrm{F}$ & 55.9998 & 58.6206 & 58.6563 & 59.2933 & 60.4663 & 61.1476 & 0.00788 & 0.01317 & 0.01779 & 0.00528 & 0.01181 & 0.02304 \\
\hline$F_{\text {af }}$ & Ibs & -0.0032 & 0.31135 & 0.61575 & 0.86821 & 1.09504 & 1.27451 & 0.10714 & 0.76444 & 0.84331 & 0.86859 & 0.87235 & 0.80847 \\
\hline $\mathrm{M}_{\mathrm{af}}$ & in-lbs & 0.08715 & 5.35284 & 16.9424 & 33.3166 & 51.3942 & 67.2031 & 0.72839 & 4.05047 & 4.31007 & 4.60359 & 4.42811 & 4.21738 \\
\hline$\omega$ & $\mathrm{rpm}$ & 0 & 20248 & 26744 & 31173 & 34760 & 37536 & 0 & 187 & 114 & 128 & 56 & 37 \\
\hline$V_{p s}$ & Volts & 16.7114 & 16.7243 & 16.7307 & 16.7415 & 16.7404 & 16.7531 & 0.01895 & 0.02217 & 0.02368 & 0.02416 & 0.02449 & 0.02417 \\
\hline $\mathrm{I}_{\mathrm{ps}}$ & Amps & -0.07667 & 5.94795 & 12.0112 & 18.0039 & 23.9355 & 29.7297 & 0.01635 & 0.01902 & 0.01948 & 0.02057 & 0.02085 & 0.02076 \\
\hline$T_{f, u}$ & ${ }^{\circ} \mathrm{F}$ & 59.779 & 56.1688 & 55.9962 & 56.3239 & 56.8341 & 57.1783 & 0.00381 & 0.00502 & 0.00353 & 0.00436 & 0.00483 & 0.00595 \\
\hline$P_{s, u f}$ & psf & -5.79975 & -8.423 & -9.88748 & -11.6668 & -13.3745 & -14.8234 & 0.003 & 0.02522 & 0.02939 & 0.03191 & 0.03582 & 0.03971 \\
\hline$P_{s, d f}$ & psf & -5.70705 & 6.92306 & 14.9485 & 21.9981 & 28.4376 & 33.9786 & 0.00332 & 0.016 & 0.02209 & 0.0249 & 0.02856 & 0.03038 \\
\hline$P_{s, o s}$ & psf & -5.74733 & -6.40649 & -6.63728 & -6.59664 & -6.77326 & -6.96417 & 0.00315 & 0.00485 & 0.00784 & 0.00508 & 0.00334 & 0.00339 \\
\hline$P_{s, c s}$ & psf & -5.7154 & -6.48584 & -6.5231 & -6.41007 & -6.54816 & -6.69374 & 0.00308 & 0.0071 & 0.01063 & 0.00638 & 0.00506 & 0.00563 \\
\hline$P_{\Delta, G}$ & psf & -0.00512 & 0.33019 & 0.7653 & 1.16026 & 1.13205 & 1.08702 & 0.00485 & 0.01516 & 0.01981 & 0.01061 & 0.00972 & 0.01092 \\
\hline $\mathrm{T}_{\mathrm{m}}$ & ${ }^{\circ} \mathrm{F}$ & 127.486 & 109.569 & 108.579 & 119.637 & 133.897 & 144.856 & 0.00967 & 0.03767 & 0.02053 & 0.02705 & 0.02495 & 0.01834 \\
\hline$A_{x 1}$ & g's & 0.00911 & 0.00869 & 0.01065 & 0.02728 & 0.04173 & 0.05171 & 0.01759 & 0.02455 & 0.03168 & 0.03704 & 0.04119 & 0.0417 \\
\hline$A_{\times 2}$ & g's & 0.00761 & 0.0097 & 0.01127 & 0.01356 & 0.01493 & 0.01827 & 0.01742 & 0.02448 & 0.02791 & 0.02696 & 0.02922 & 0.03417 \\
\hline$A_{y}$ & g's & -0.97928 & -0.97953 & -0.95707 & -0.85528 & -0.7286 & -0.57419 & 0.01995 & 0.02139 & 0.03339 & 0.0886 & 0.12365 & 0.14343 \\
\hline$A_{2}$ & g's & 0.24112 & 0.24123 & 0.24165 & 0.24186 & 0.24051 & 0.23852 & 0.01759 & 0.04823 & 0.05067 & 0.04494 & 0.05035 & 0.05366 \\
\hline
\end{tabular}




\begin{tabular}{|c|c|c|c|c|c|c|c|c|c|c|c|c|c|}
\hline C5 & units & OW & $100 \mathrm{~W}$ & $200 \mathrm{~W}$ & $300 \mathrm{~W}$ & $400 \mathrm{~W}$ & $500 \mathrm{~W}$ & $\begin{array}{c}\text { OW std } \\
\text { dev }\end{array}$ & $\begin{array}{c}100 \mathrm{~W} \\
\text { std dev }\end{array}$ & $\begin{array}{c}200 \mathrm{~W} \\
\text { std dev }\end{array}$ & $\begin{array}{c}300 \mathrm{~W} \\
\text { std dev }\end{array}$ & $\begin{array}{c}400 \mathrm{~W} \\
\text { std dev }\end{array}$ & $\begin{array}{c}500 \mathrm{~W} \\
\text { std dev }\end{array}$ \\
\hline $\operatorname{Re}_{L}$ & & 1415281 & 1413982 & 1419127 & 1419570 & 1420405 & 1421670 & 0 & 0 & 0 & 0 & 0 & 0 \\
\hline G & in & 0.80282 & 0.80282 & 0.80282 & 0.80279 & 0.8028 & 0.80279 & $2 \mathrm{E}-05$ & $2.2 \mathrm{E}-05$ & $2.2 \mathrm{E}-05$ & $2.2 \mathrm{E}-05$ & $2.2 \mathrm{E}-05$ & $2.2 \mathrm{E}-05$ \\
\hline$P_{\infty}$ & psi & 14.7248 & 14.7248 & 14.7248 & 14.7248 & 14.7248 & 14.7248 & 0.00982 & 0.00982 & 0.00982 & 0.00982 & 0.00982 & 0.00982 \\
\hline$T_{\infty}$ & ${ }^{\circ} \mathrm{F}$ & 56.2 & 56.2 & 56.2 & 56.2 & 56.2 & 56.2 & 0.2 & 0.2 & 0.2 & 0.2 & 0.2 & 0.2 \\
\hline$\rho_{\infty}$ & slugs $/ \mathrm{ft}^{3}$ & 0.00235 & 0.00235 & 0.00235 & 0.00235 & 0.00235 & 0.00235 & $1.8 \mathrm{E}-06$ & $1.8 \mathrm{E}-06$ & $1.8 \mathrm{E}-06$ & $1.8 \mathrm{E}-06$ & $1.8 \mathrm{E}-06$ & $1.8 \mathrm{E}-06$ \\
\hline$q_{\infty}$ & psf & 5.78326 & 5.77265 & 5.81474 & 5.81837 & 5.82521 & 5.83559 & 0.00322 & 0.00315 & 0.00297 & 0.00292 & 0.00299 & 0.00286 \\
\hline $\mathrm{U}_{\infty}$ & $\mathrm{ft} / \mathrm{s}$ & 70.1606 & 70.0962 & 70.3513 & 70.3732 & 70.4146 & 70.4773 & 0.06657 & 0.06602 & 0.06489 & 0.06454 & 0.06504 & 0.06418 \\
\hline $\mathrm{F}_{\mathrm{a}}$ & lbs & -0.35238 & -0.22338 & 0.00757 & 0.24103 & 0.45552 & 0.69112 & 0.0012 & 0.00191 & 0.00189 & 0.00165 & 0.00178 & 0.00159 \\
\hline$C_{a, p}$ & lbs & -0.00786 & -0.0083 & -0.00347 & -0.00957 & -0.01151 & -0.01396 & 0.00151 & 0.00114 & 0.00117 & 0.00112 & 0.00111 & 0.0011 \\
\hline$\dot{\mathrm{m}}$ & slugs/s & 0 & 0.0032 & 0.00445 & 0.00536 & 0.00603 & 0.00653 & $1.5 \mathrm{E}-21$ & $1.4 \mathrm{E}-05$ & $1.8 \mathrm{E}-05$ & $1.6 \mathrm{E}-05$ & $1.7 \mathrm{E}-05$ & $1.8 \mathrm{E}-05$ \\
\hline$\rho_{\mathrm{e}}$ & slugs $/ \mathrm{ft}^{3}$ & 0.00235 & 0.00234 & 0.00235 & 0.00235 & 0.00236 & 0.00236 & $4.8 \mathrm{E}-09$ & $4.8 \mathrm{E}-09$ & $4.9 \mathrm{E}-09$ & 4.9E-09 & $5.1 \mathrm{E}-09$ & $5.1 \mathrm{E}-09$ \\
\hline $\mathrm{q}_{\mathrm{e}, \mathrm{u}}$ & psf & -0.55163 & 6.67499 & 12.9195 & 18.6712 & 23.6363 & 27.6887 & 0.00744 & 0.01366 & 0.02483 & 0.02358 & 0.02895 & 0.03177 \\
\hline$U_{e, u}$ & $\mathrm{ft} / \mathrm{s}$ & 0 & 75.4691 & 104.887 & 125.936 & 141.61 & 153.247 & $1.8 \mathrm{E}-17$ & 0.15405 & 0.2015 & 0.15934 & 0.17403 & 0.17645 \\
\hline $\mathrm{P}_{\mathrm{t}, \mathrm{e}}$ & psf & 2114.27 & 2123.23 & 2130.4 & 2136.82 & 2142.21 & 2146.59 & 0.05278 & 0.05302 & 0.05635 & 0.05598 & 0.05811 & 0.05925 \\
\hline $\mathrm{T}_{\mathrm{e}}$ & ${ }^{\circ} \mathrm{F}$ & 55.5258 & 58.2133 & 58.9123 & 59.1976 & 59.8998 & 60.8254 & 0.00778 & 0.01393 & 0.01102 & 0.01372 & 0.02067 & 0.017 \\
\hline$F_{\text {af }}$ & Ibs & 0.03571 & 0.35518 & 0.65125 & 0.90869 & 1.12963 & 1.32099 & 0.10537 & 0.83799 & 0.90857 & 0.92263 & 0.92437 & 0.8486 \\
\hline $\mathrm{M}_{\mathrm{af}}$ & in-lbs & 0.07411 & 5.1558 & 15.7204 & 32.3581 & 48.2459 & 65.4429 & 0.73077 & 4.69043 & 5.11149 & 5.5381 & 5.34354 & 4.98246 \\
\hline$\omega$ & $\mathrm{rpm}$ & 0 & 20041 & 26566 & 31110 & 34681 & 37480 & 0 & 71 & 105 & 92 & 55 & 43 \\
\hline$V_{p s}$ & Volts & 16.7149 & 16.7237 & 16.7259 & 16.738 & 16.7444 & 16.7546 & 0.01927 & 0.02276 & 0.0242 & 0.02421 & 0.02433 & 0.02397 \\
\hline $\mathrm{I}_{\mathrm{ps}}$ & Amps & -0.07736 & 6.0043 & 11.8688 & 17.9469 & 23.9078 & 29.7401 & 0.01634 & 0.01941 & 0.0204 & 0.02082 & 0.02048 & 0.02062 \\
\hline$T_{f, u}$ & ${ }^{\circ} \mathrm{F}$ & 58.5196 & 55.463 & 55.8878 & 55.9483 & 56.1899 & 56.9236 & 0.00236 & 0.00591 & 0.00577 & 0.00455 & 0.00371 & 0.00477 \\
\hline$P_{s, u f}$ & psf & -5.80861 & -8.50031 & -9.95283 & -11.8589 & -13.6979 & -15.3123 & 0.00289 & 0.02198 & 0.02839 & 0.03113 & 0.03446 & 0.03808 \\
\hline$P_{s, d f}$ & psf & -5.71748 & 6.11622 & 14.8229 & 21.878 & 28.3307 & 33.8641 & 0.00312 & 0.01506 & 0.02167 & 0.0246 & 0.02776 & 0.02996 \\
\hline$P_{s, o s}$ & psf & -5.76166 & -6.19003 & -6.25253 & -6.29337 & -6.40986 & -6.54976 & 0.00303 & 0.00353 & 0.0054 & 0.0028 & 0.00271 & 0.00269 \\
\hline$P_{s, c s}$ & psf & -5.73439 & -6.30162 & -6.2789 & -6.21518 & -6.32124 & -6.45827 & 0.00299 & 0.00461 & 0.01023 & 0.00623 & 0.00675 & 0.00724 \\
\hline$P_{\Delta, G}$ & psf & 0.02119 & 1.05575 & 1.04288 & 1.29131 & 1.35414 & 1.36356 & 0.00517 & 0.00611 & 0.01313 & 0.00593 & 0.00541 & 0.00547 \\
\hline $\mathrm{T}_{\mathrm{m}}$ & ${ }^{\circ} \mathrm{F}$ & 126.59 & 101.598 & 107.302 & 117.894 & 129.146 & 140.731 & 0.00685 & 0.06109 & 0.02634 & 0.03483 & 0.01191 & 0.01681 \\
\hline$A_{x 1}$ & g's & 0.00886 & 0.00782 & 0.0149 & 0.0352 & 0.04676 & 0.05352 & 0.01732 & 0.02337 & 0.03308 & 0.0381 & 0.04103 & 0.04052 \\
\hline$A_{\times 2}$ & g's & 0.00683 & 0.00879 & 0.01092 & 0.0127 & 0.01458 & 0.01877 & 0.01742 & 0.02371 & 0.02578 & 0.02642 & 0.03119 & 0.03705 \\
\hline$A_{y}$ & g's & -0.97944 & -0.97901 & -0.94354 & -0.80656 & -0.66467 & -0.50234 & 0.01972 & 0.02115 & 0.046 & 0.12036 & 0.14607 & 0.1708 \\
\hline$A_{2}$ & g's & 0.24154 & 0.24061 & 0.24163 & 0.24166 & 0.24118 & 0.23877 & 0.02846 & 0.03204 & 0.05264 & 0.03738 & 0.04889 & 0.05602 \\
\hline
\end{tabular}




\section{APPENDIX C - MATLAB DATA PROCESSING FUNCTIONS}

These functions are included so that all data analysis techniques are transparent.

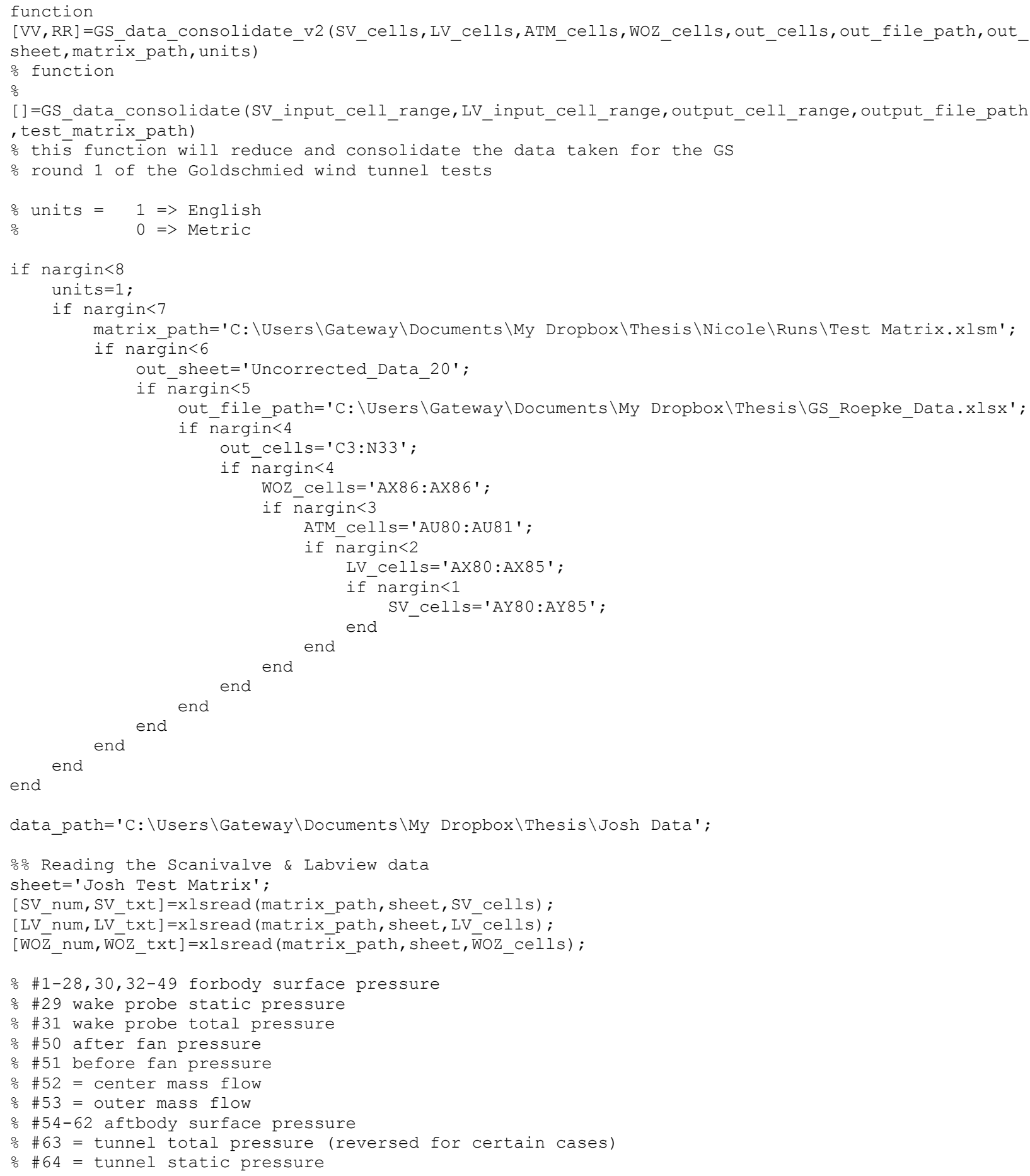




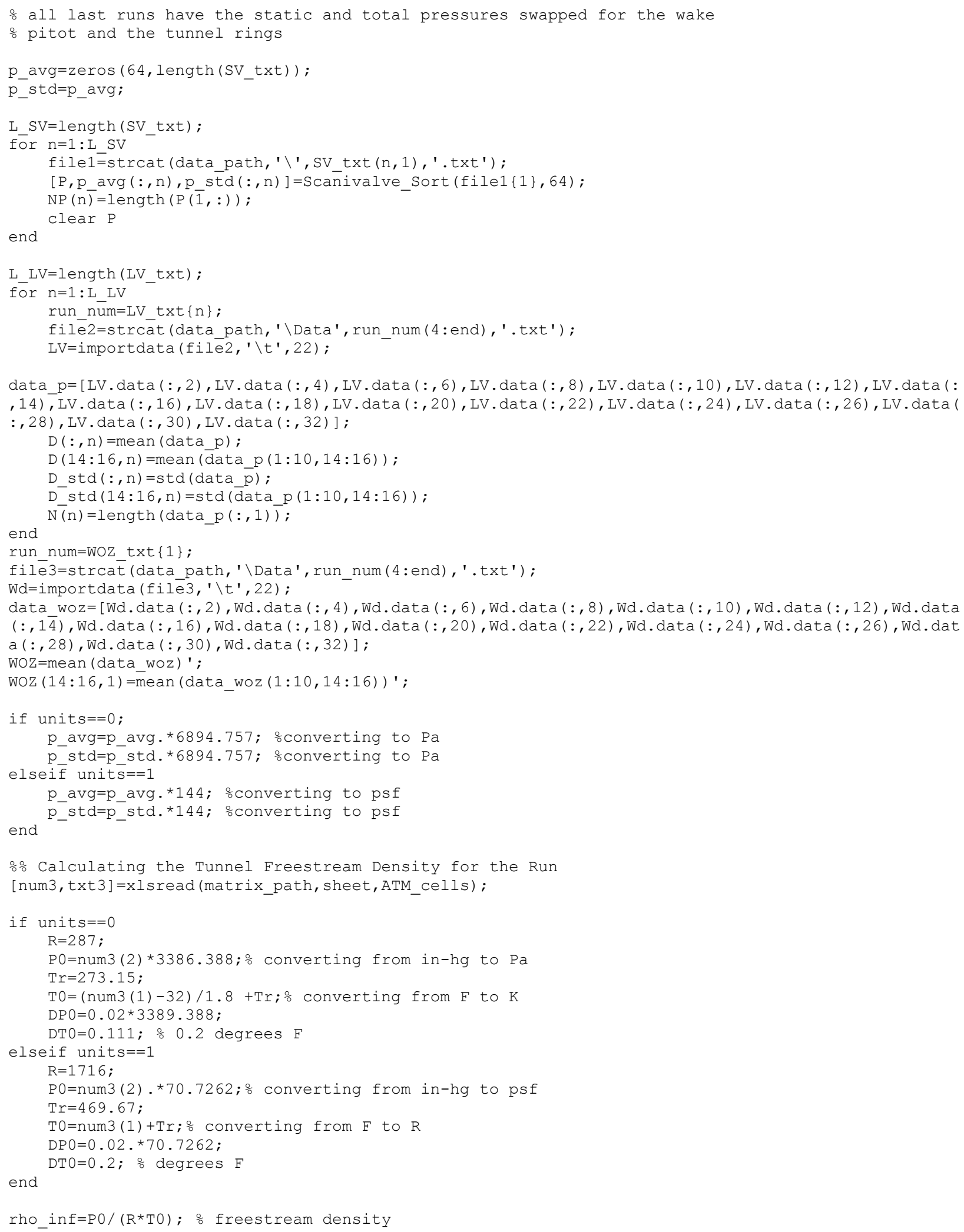




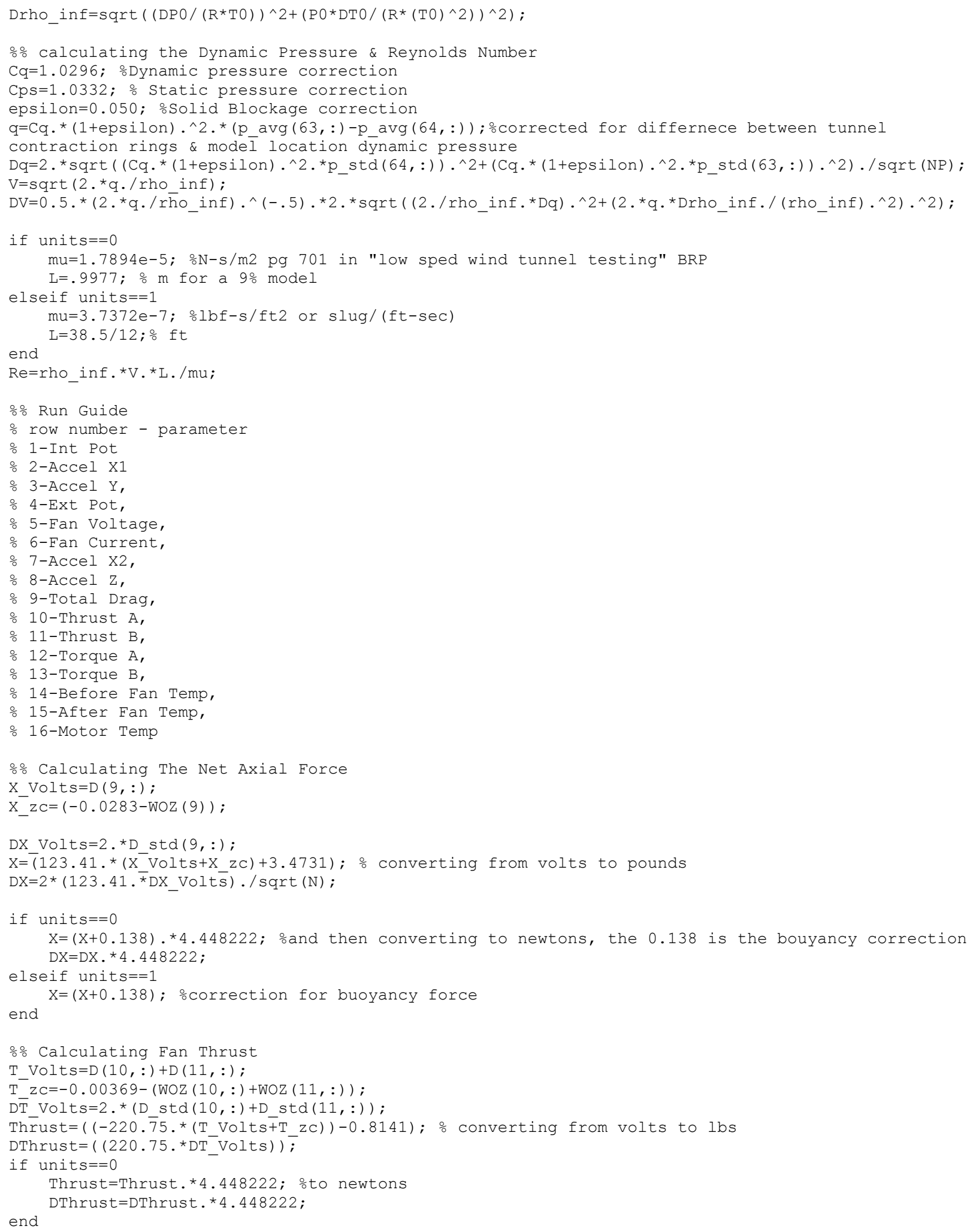




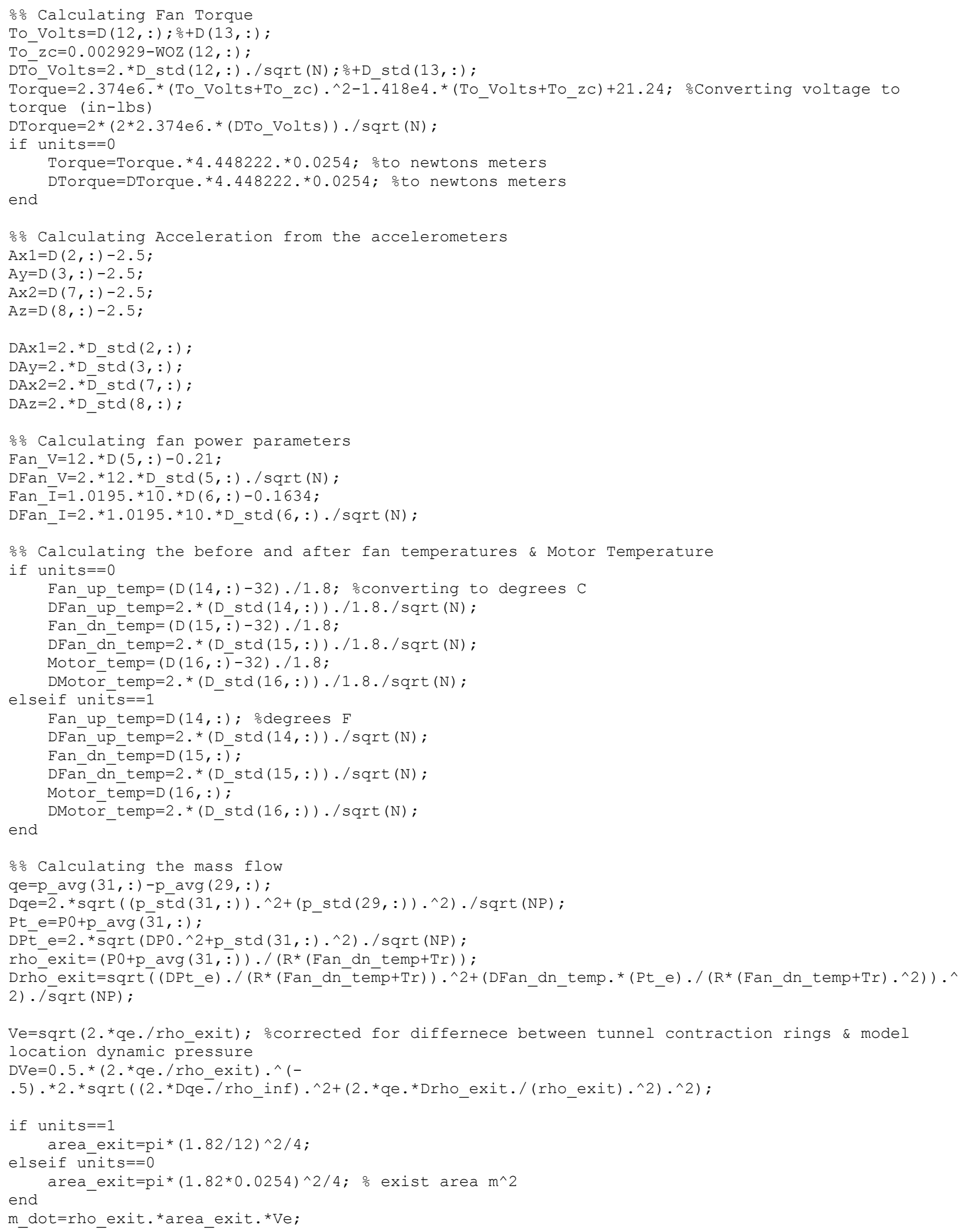




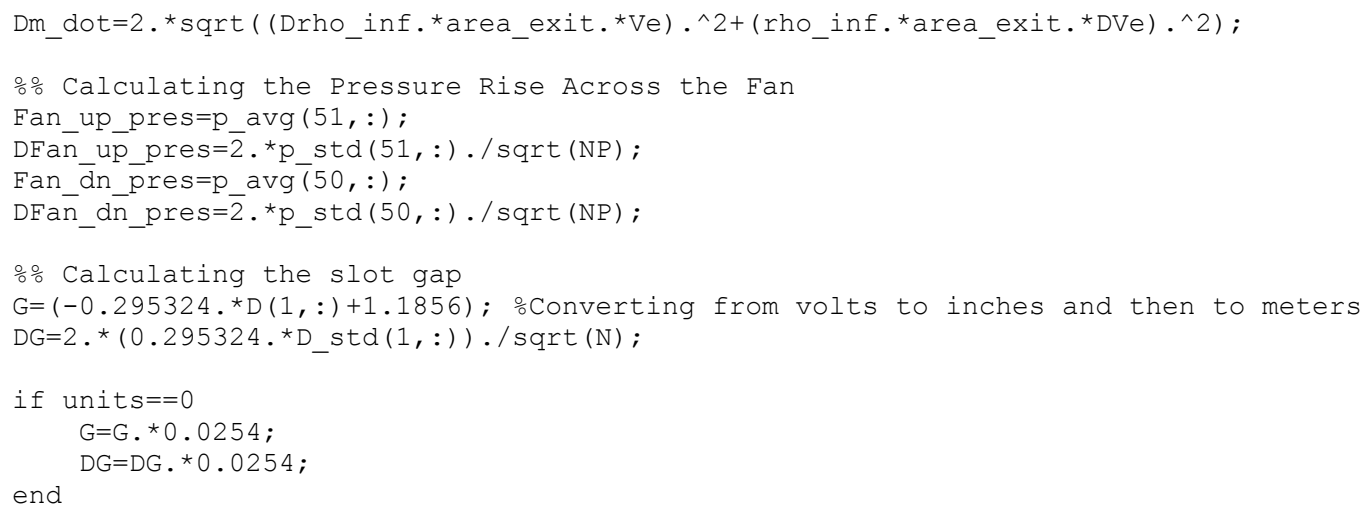




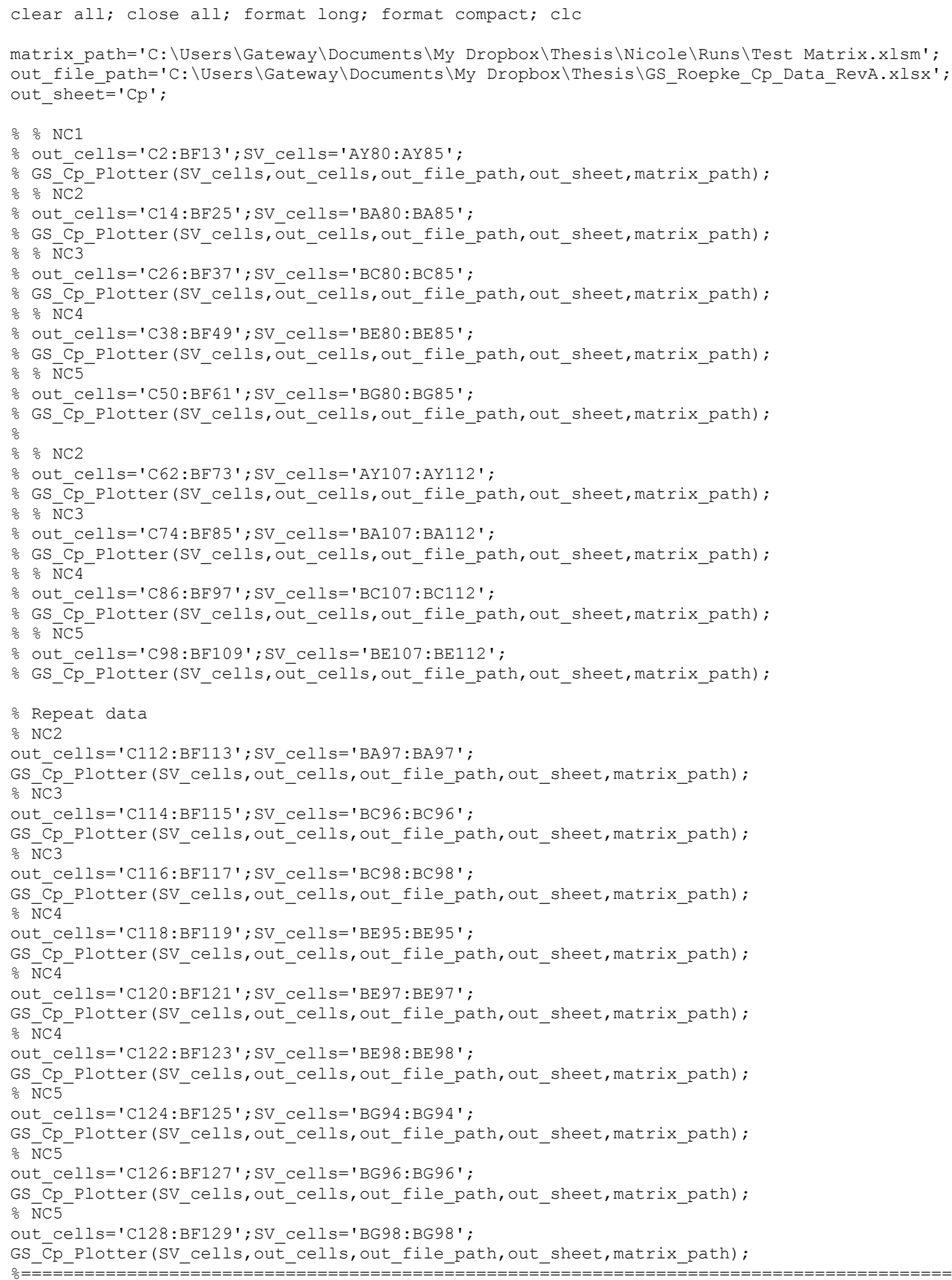




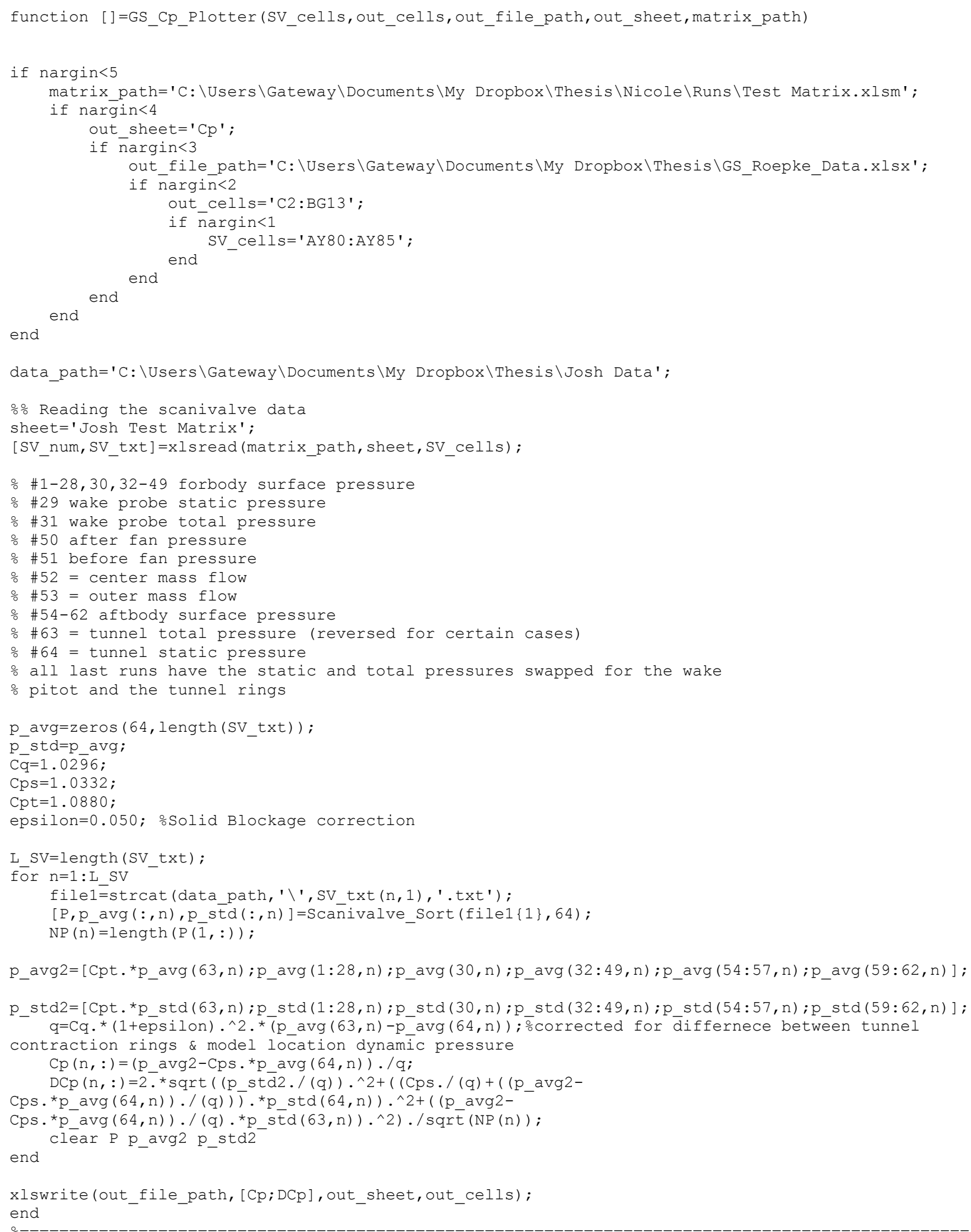




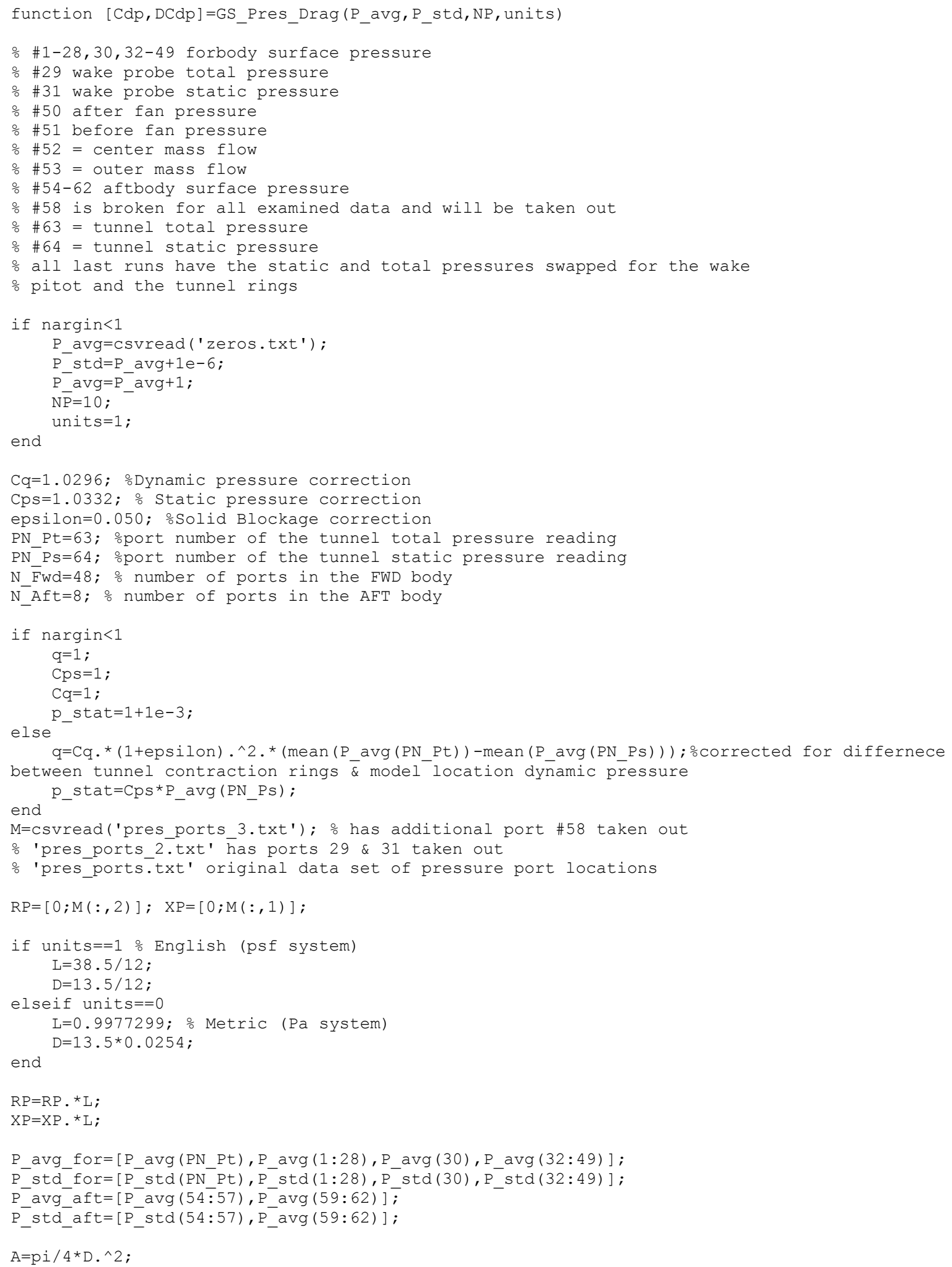




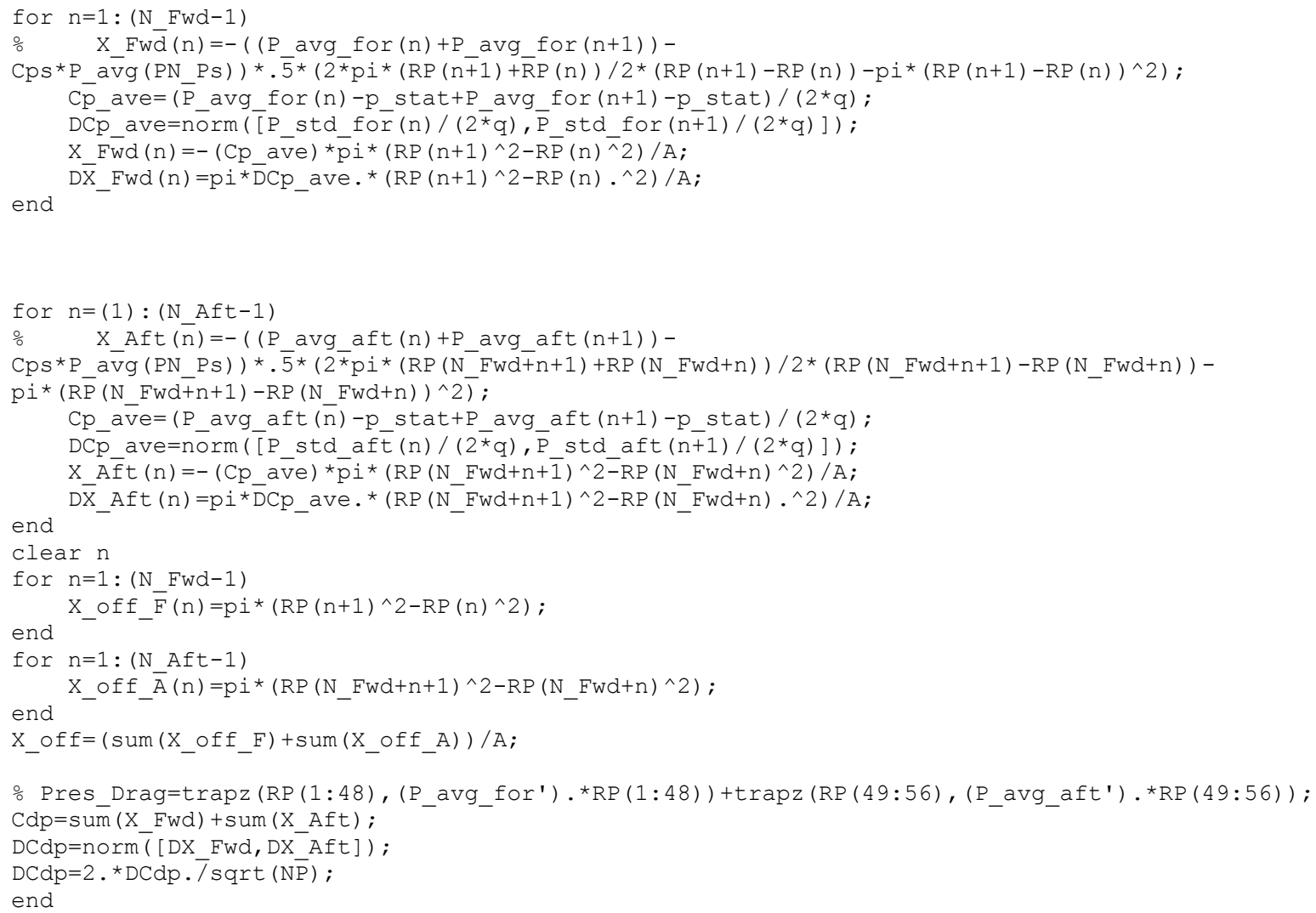




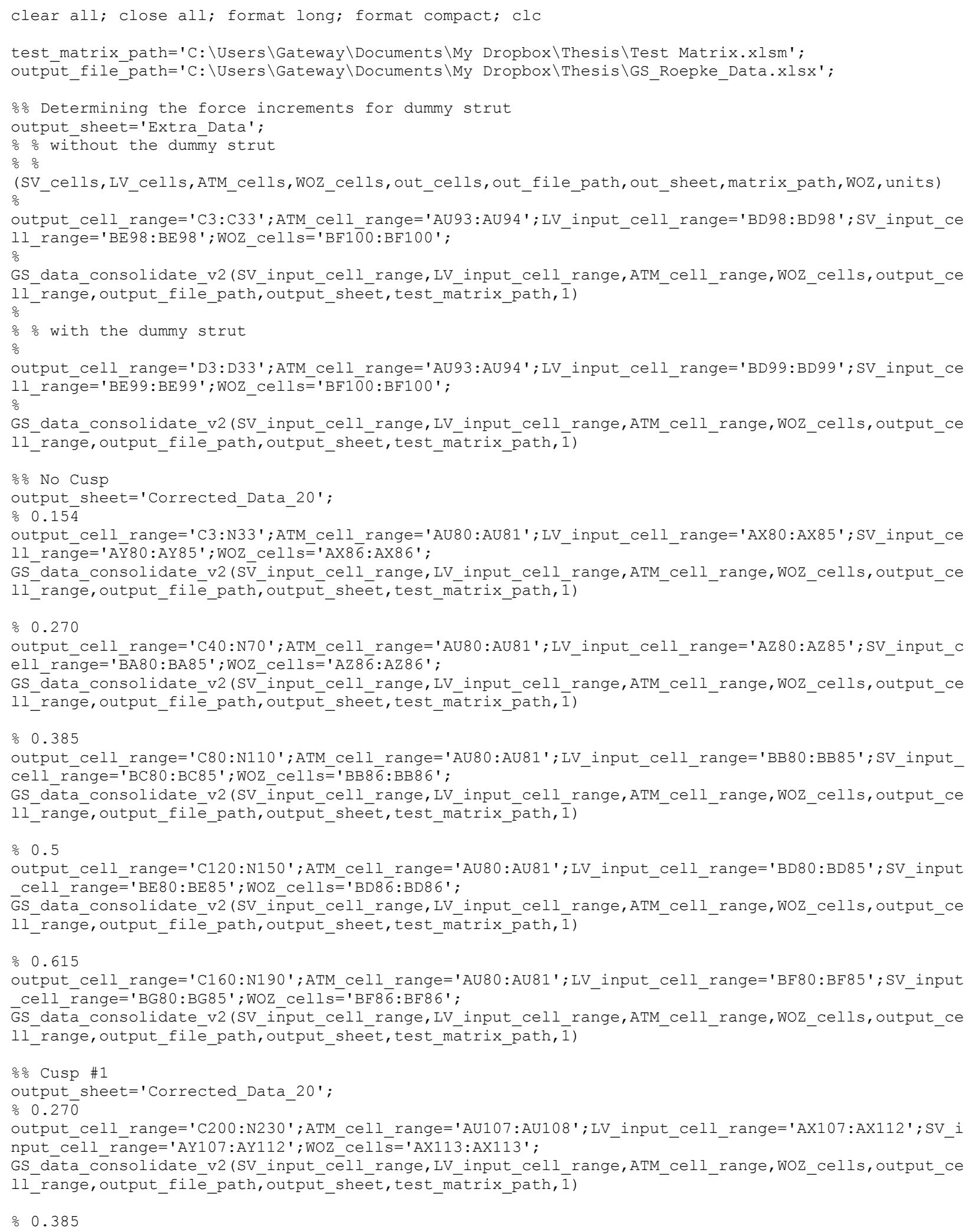


output_cell_range='C240:N270';ATM_cell_range='AU107:AU108'; LV_input_cell_range='AZ107:AZ112';SV_i nput_céll_range='BA107:BA112'; WOZ_cells's='AZ113:AZ113';

GS_dāta_consolidate_v2 (SV_input_cêll_range, LV_input_cell_range, ATM_cell_range, woz_cells, output_ce

ll_range, output_filē_path, output_sheet, test_matrix_path, $\overline{1}$ )

$\div 0.5$

output_cell_range='C280:N310';ATM_cell_range='AU107:AU108'; LV_input_cell_range= 'BB107:BB112'; SV_i nput cell range='BC107:BC112'; WOZ cells='BB113:BB113';

GS_dāta_consolidate_v2(SV_input_cēll_range, LV_input_cell_range,ATM_cell_range, woz_cells, output_ce ll_rangēe, output_filē_path, output_sheèt, test_mätrix_p̄ath, $\overline{1})$

$\circ 0.615$

output_cell_range='C320:N350'; ATM_cell_range='AU107:AU108'; LV_input_cell_range='BD107:BD112'; SV_i nput céll rānge='BE107:BE112'; WOZ_cells's='BD113:BD113';

GS_data_consolidate_v2(SV_input_cell_range,LV_input_cell_range,ATM_cell_range, Woz_cells, output_ce

l1_rangē,output_filēepath, output_sheèt, test_mätrix_path, $\overline{1}$ )

$\therefore$ 응 redundant data points

output sheet='Extra Data';

$\therefore 0.27 \overline{0} \# 1$

output_cell_range='F3:G33';ATM_cell_range='AU93:AU94';LV_input_cell_range='AZ97:AZ97';SV_input_ce 11_range ='BĀ97:BA97'; WOZ_cells='BF1000:BF100';

GS_data_consolidate_v2 (SV__input_cell_range, LV_input_cell_range, ATM_cell_range, woz_cells, output_ce

l1_range, output_filē_path, output_sheet, test_matrix_path, $\overline{1}$ )

$\div 0.385 \# 1$

output_cell_range='H3:I33';ATM_cell_range='AU93:AU94'; LV_input_cell_range='BB96:BB96';SV_input_ce 11 range $=$ 'BC $\overline{\mathrm{C}} 96: \mathrm{BC} 96^{\prime} ;$ WOZ cells $=$ 'BF1000:BF100';

GS_data_consolidate_v2 (SV _input_cell_range, LV_input_cell_range,ATM_cell_range, woz_cells, output_ce

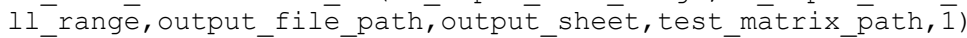

$\div 0.385 \# 2$

output_cell_range='J3:K33' ; ATM_cell_range='AU93:AU94'; LV_input_cell_range='BB98:BB98';SV_input_ce 11 ranḡe $=$ 'BC $98: \mathrm{BC} 98^{\prime} ; \mathrm{WOZ}$ cells $=$ 'BF1000:BF100';

GS_data_consolidate_v2 (SV_input_cell_range, LV_input_cell_range,ATM_cell_range, wOz_cells, output_ce

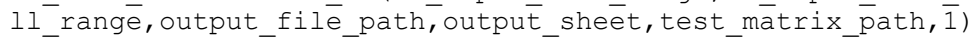

$\div 0.5$ \#1

output_cell range='L3:M33';ATM cell range='AU93:AU94'; LV input cell range='BD95:BD95';SV input_ce 11_range ='BE $95: \mathrm{BE} 95$ '; WOZ_cells='BF10̄0:BF100';

GS_data_consolidate_v2(SV__input_cell_range, LV_input_cell_range,ATM_cell_range, woz_cells, output_ce

ll_rangēe, output_filē_path, output_sheèt, test_mätrix_p̄path, $\overline{1}$ )

$\div 0.5 \# 2$

output_cell_range='N3:033';ATM_cell_range='AU93:AU94';LV_input_cell_range='BD97:BD97' ; SV_input_ce ll_ranḡe='BE $97: \mathrm{BE} 97$ '; WOZ_cells $=$ 'BF10̄0:BF100';

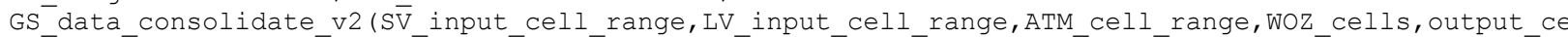

11_rangē,output_filē_path, output_sheèt,test_mätrix_path, $\overline{1}$ )

$\div 0.5 \# 3$

output_cell_range='P3:Q33';ATM_cell_range='AU93:AU94'; LV_input_cell_range='BD98:BD98' ; SV_input_ce 11 range $=$ 'BE $98: \mathrm{BE} 98$ '; WOZ cells='BF100:BF100';

GS_data_consolidate_v2 (SV__input_cell_range, LV_input_cell_range, ATM_cell_range, woz_cells, output_ce

ll_rangē, output_filē_path, output_sheet, test_matrix_path, $\overline{1}$ )

$\div 0.615 \# 1$

output_cell_range='R3:S33'; ATM_cell_range='AU93:AU94'; LV_input_cell_range='BF94:BF94';SV_input_ce

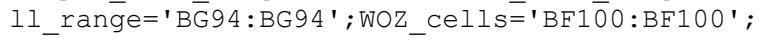

GS_data_consolidate_v2 (SV_input_cell_range, LV_input_cell_range, ATM_cell_range, wOZ_cells, output_ce

ll_range, output_filēepath, output_sheet, test_matrix_path, $\overline{1}$ )

$\div 0.615 \# 2$

output_cell_range='T3:U33';ATM_cell_range='AU93:AU94'; LV_input_cell_range='BF96:BF96';SV_input_ce 11_range $=$ 'BG96:BG96'; WOZ_cells $=$ 'BF100:BF100';

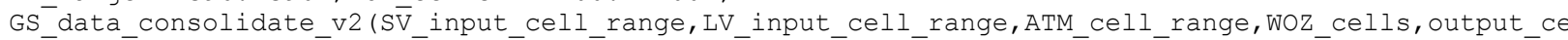

ll_rangē, output_filē_path, output_sheet, test_matrix_path, $\overline{1}$ ) 


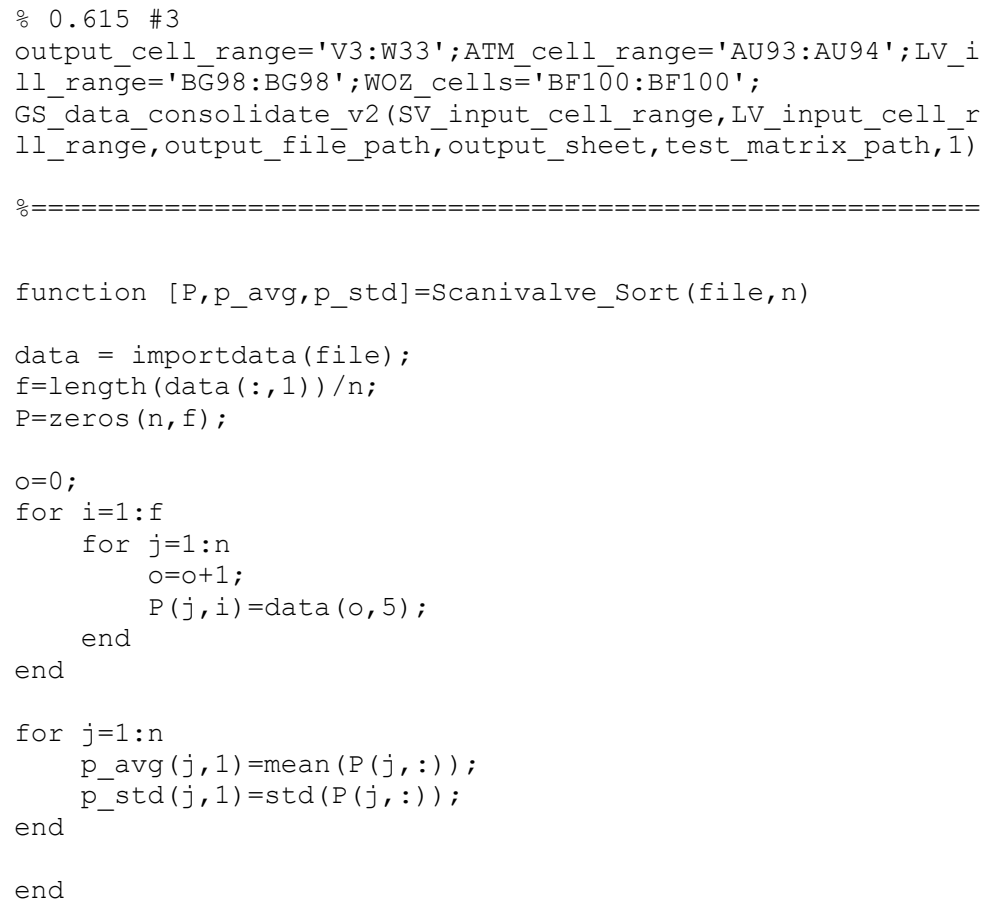




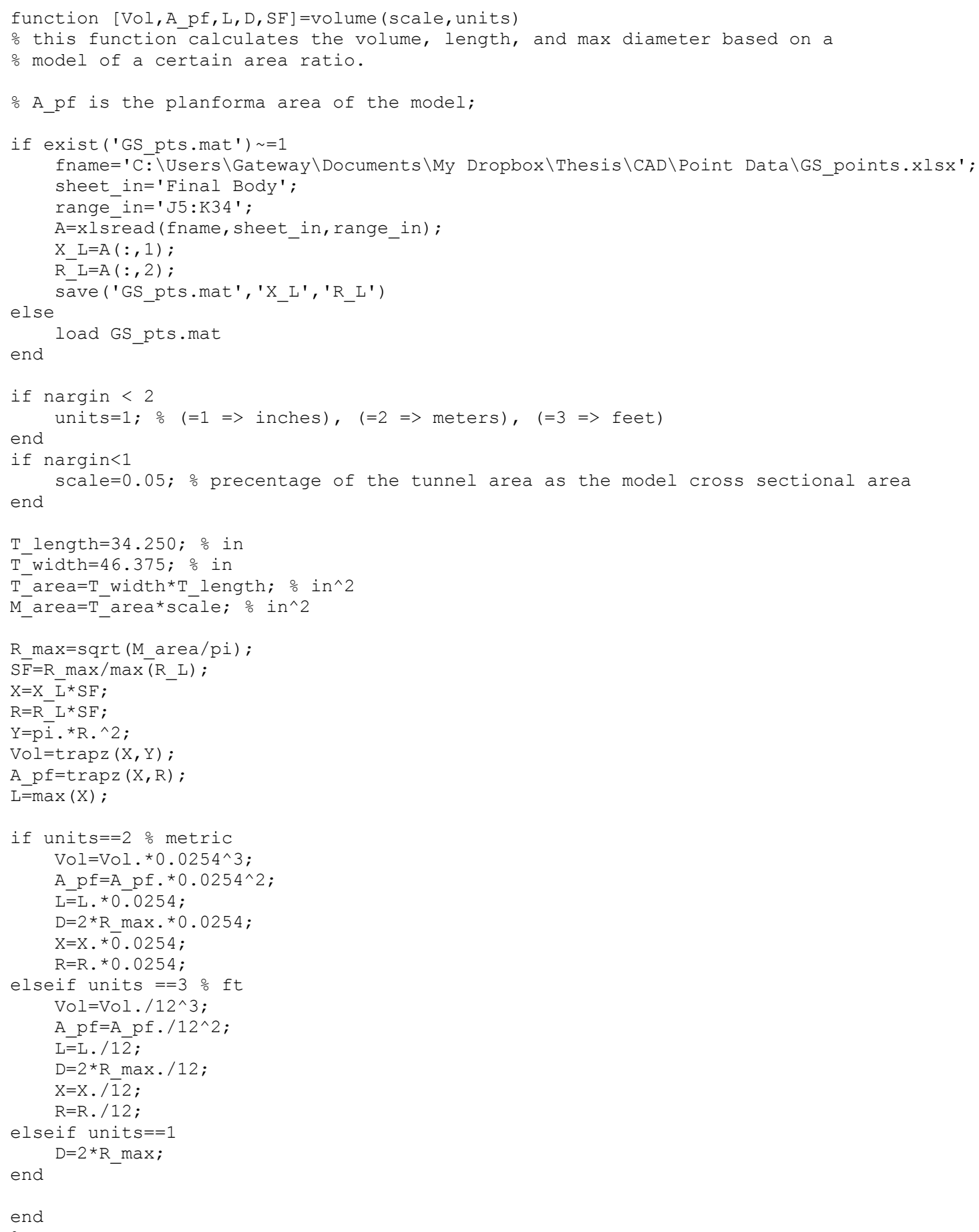




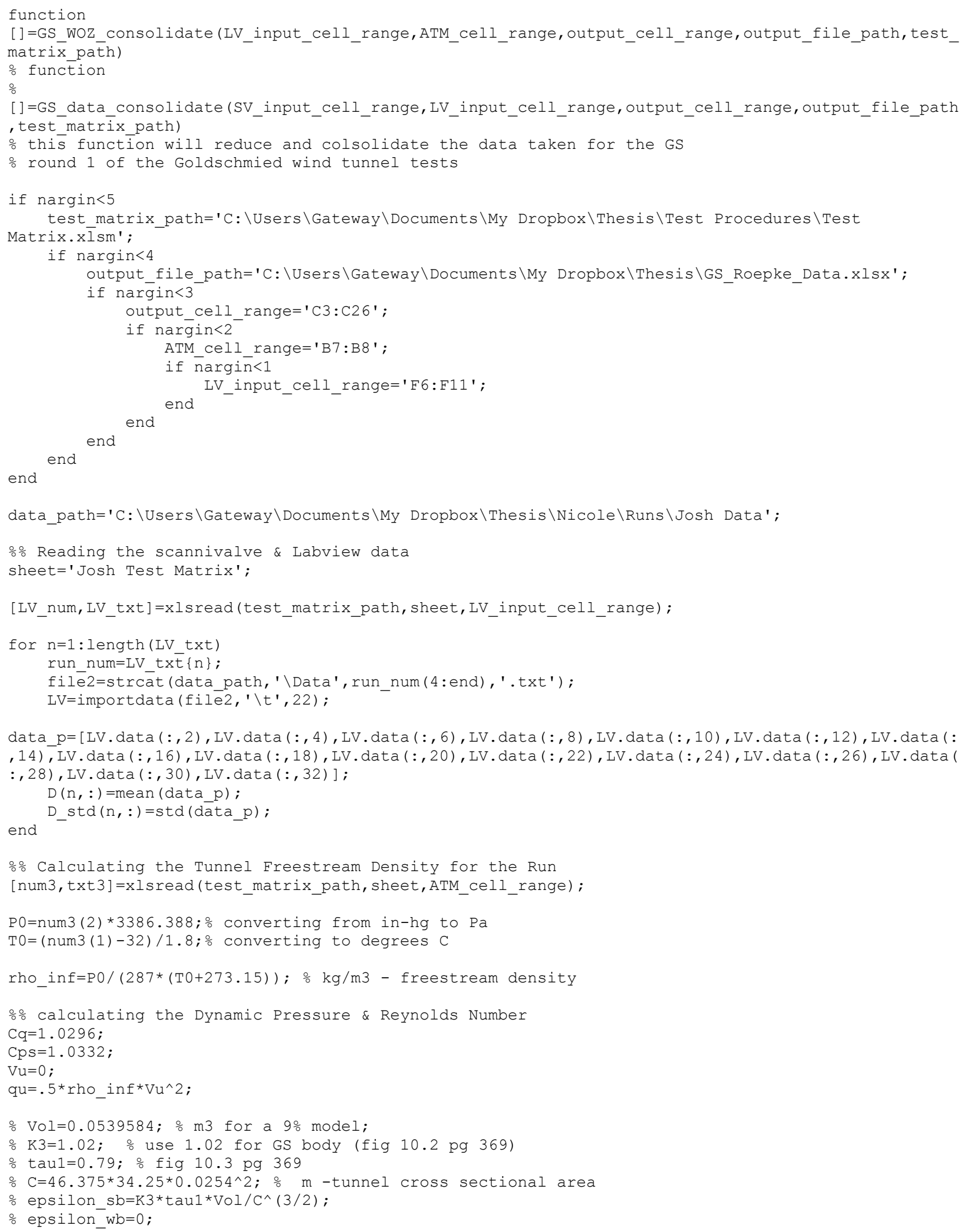




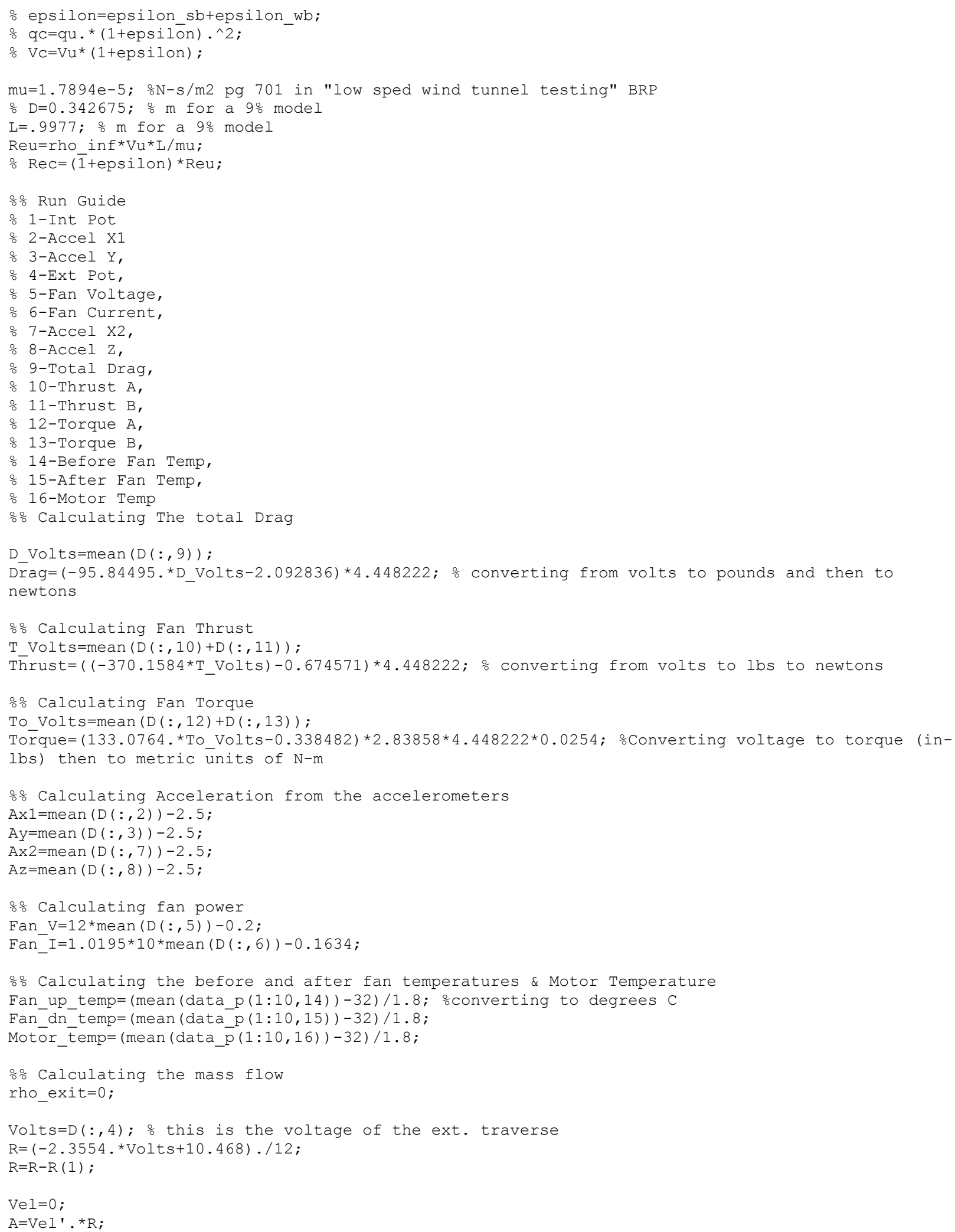


m_dot $=0$;

응 Calculating the Pressure Rise Across the Fan

Fan_up_pres $=0$;

Fan_dn_pres $=0$;

$\div \%$ Calculating the slot gap

$\mathrm{G}=(-0.283824 *$ mean $(\mathrm{D}(:, 1))+1.011823) * 0.0254$; Converting from volts to inches and then to meters

응 Calculating the pressure drag

P avg=0;

Drag pres=0;

$\because \frac{0}{0}$ Calcualting the fan speed

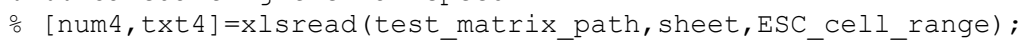

\% file4=strcat (data path, ' $\backslash$ ', txt4,' '.csv');

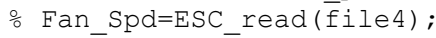

Fan $\mathrm{Spd}=0$;

응 Calculating the original mass flow port pressures

Slot_center_pres=0;

slot_outer_pres=0;

$\therefore \%$ Creating the output vector and writing it to Excel

O=[Reu G PO TO rho_inf rho_exit qu Vu Drag Drag_pres m_dot Thrust Torque Fan_Spd Fan_V Fan_I Fan_up_temp Fan_dn_temp Fan_up_pres Fan_dn_pres Slot_center_pres Slot_outer_pres Motor_temp Ax1 $\mathrm{Ax} 2 \mathrm{Ay} \mathrm{Az}]$;

xlswrite (output_file_path, ${ }^{\prime}$, output_cell_range);

end

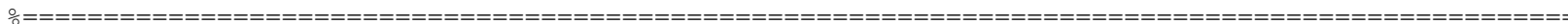




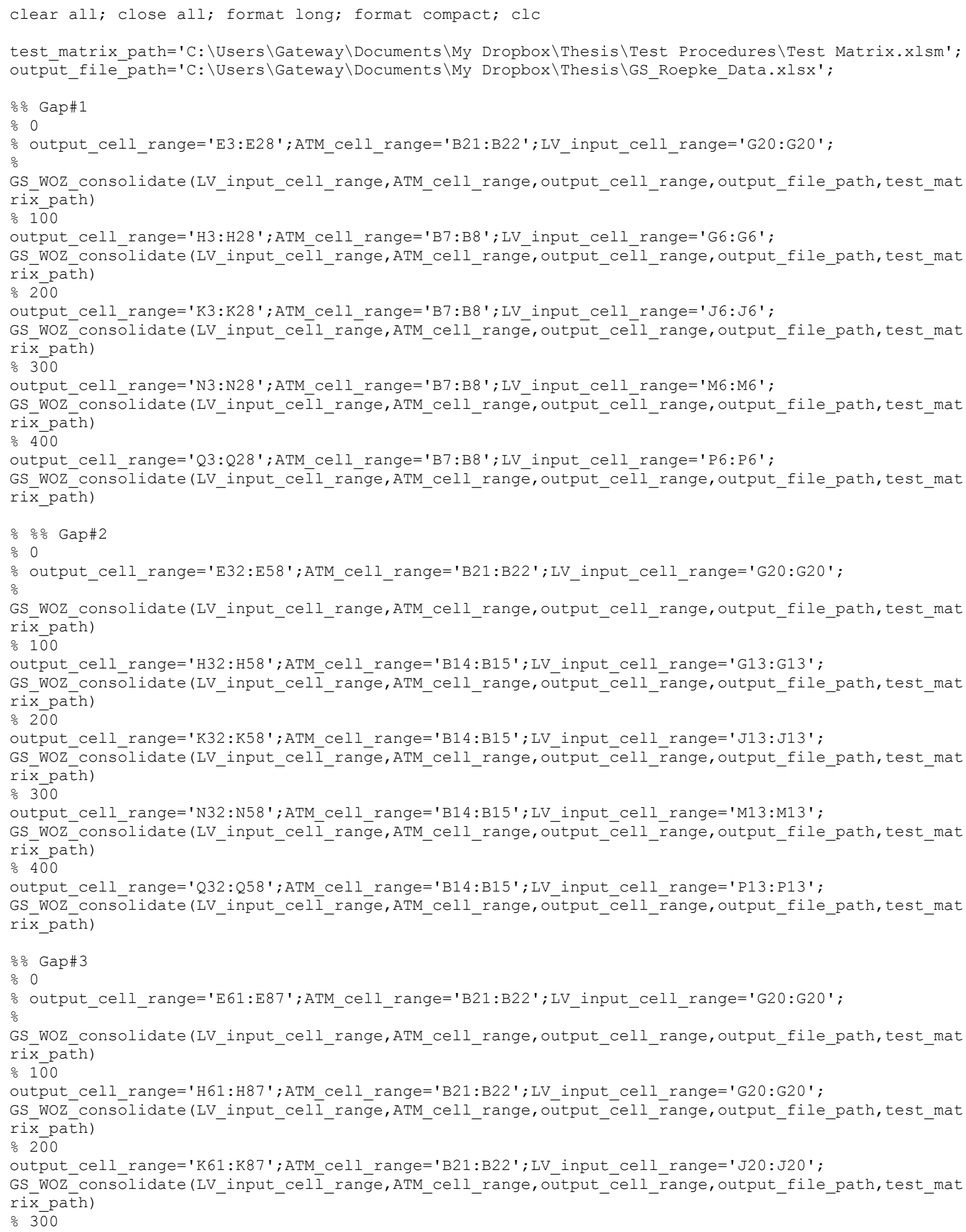




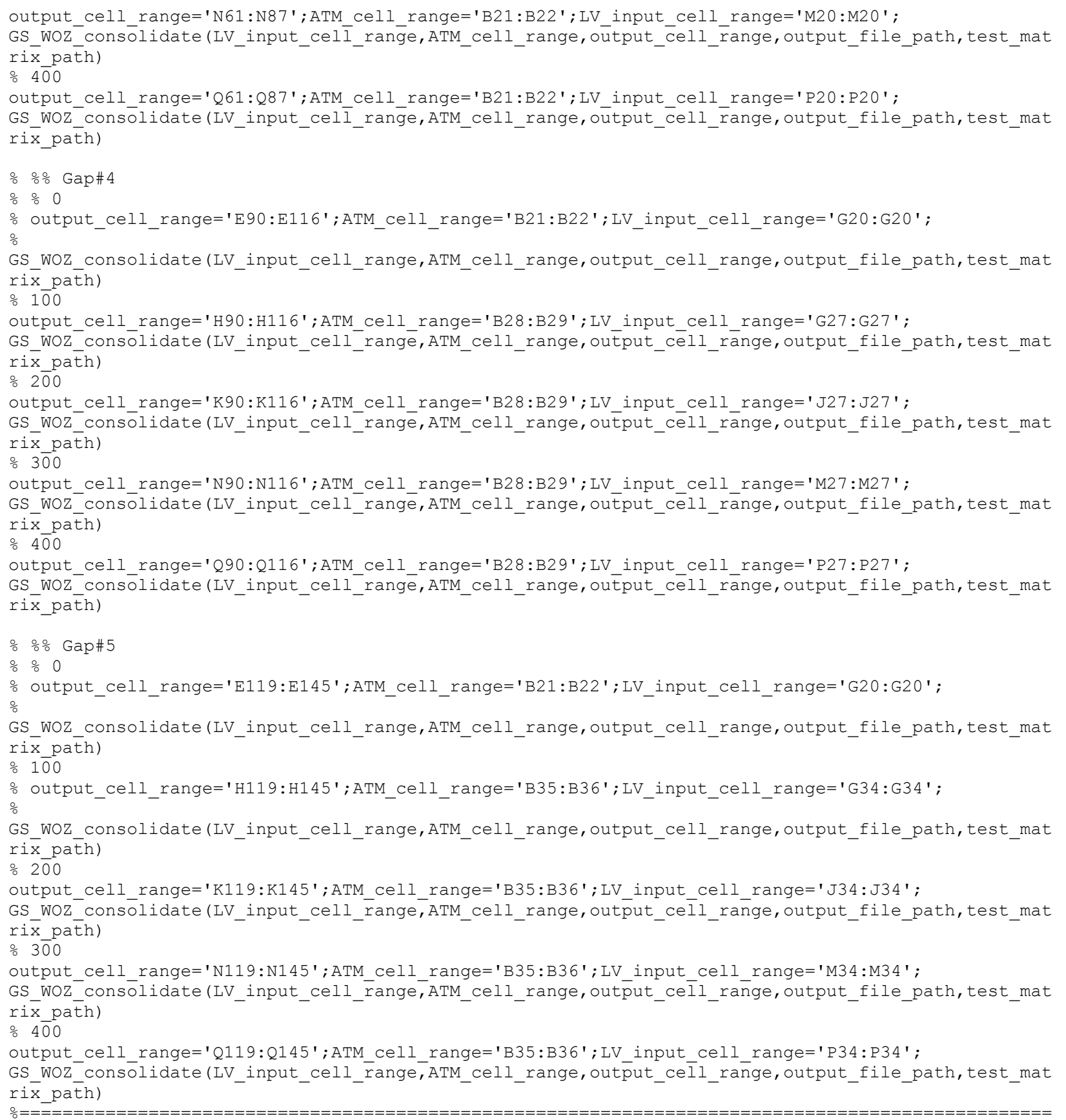




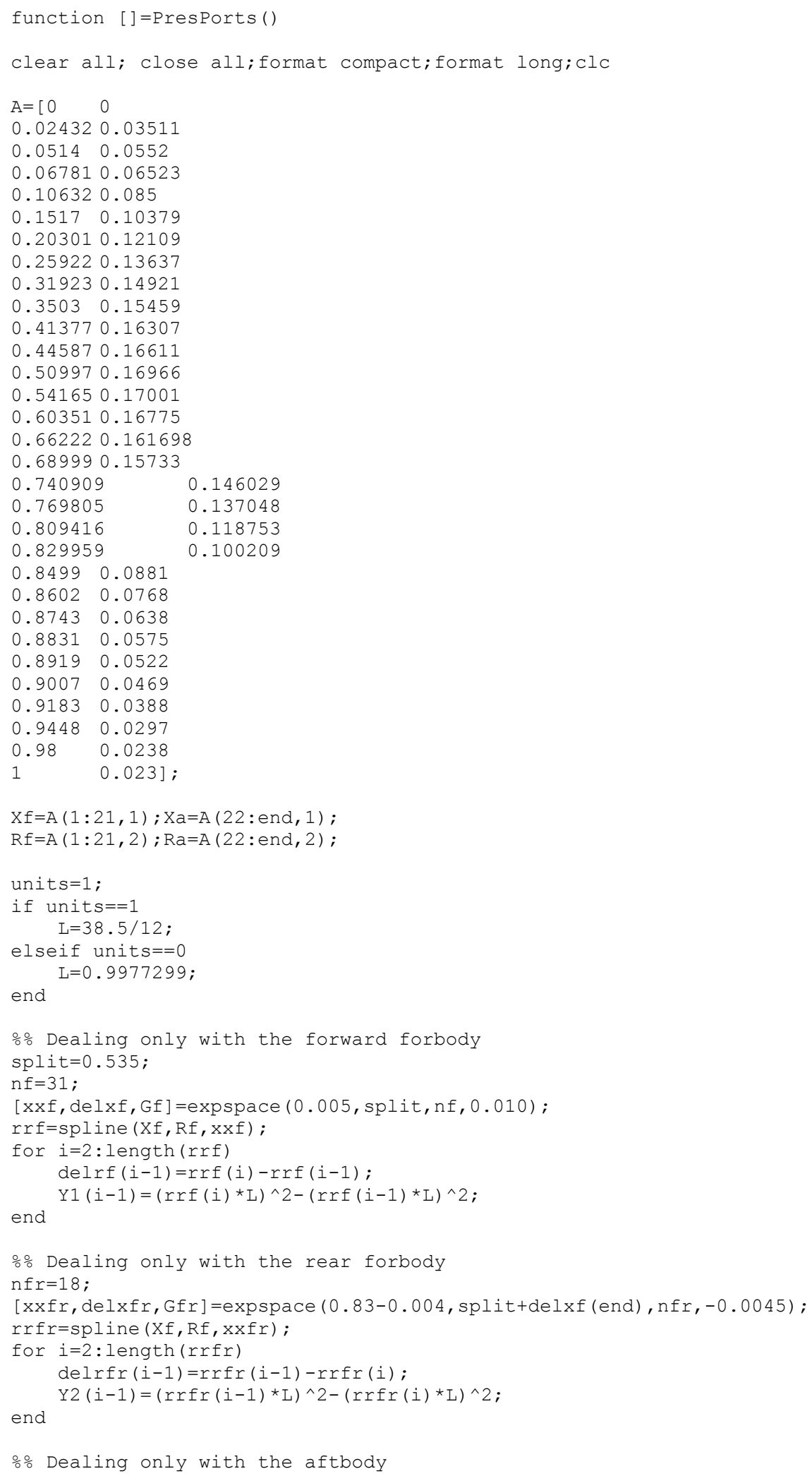




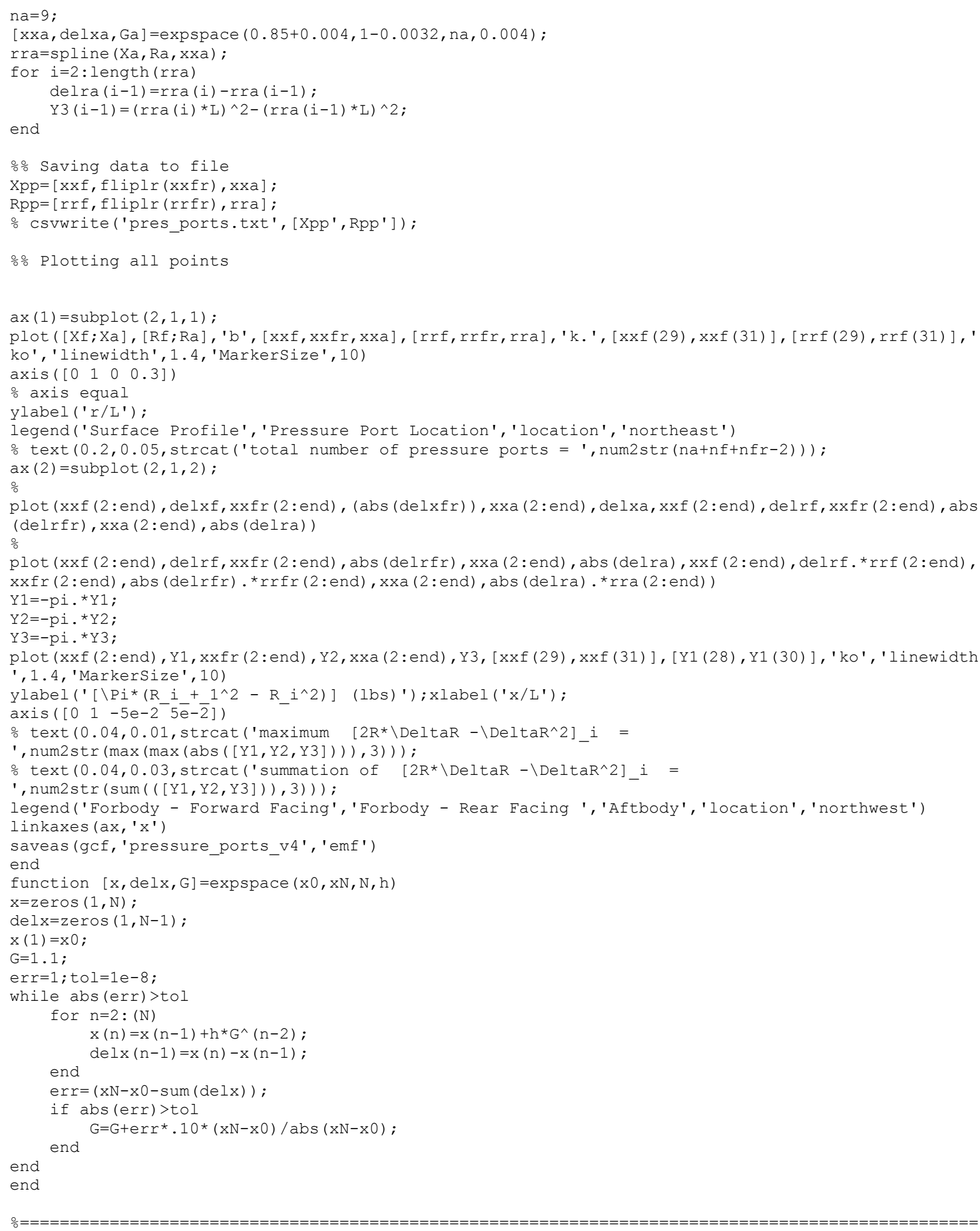




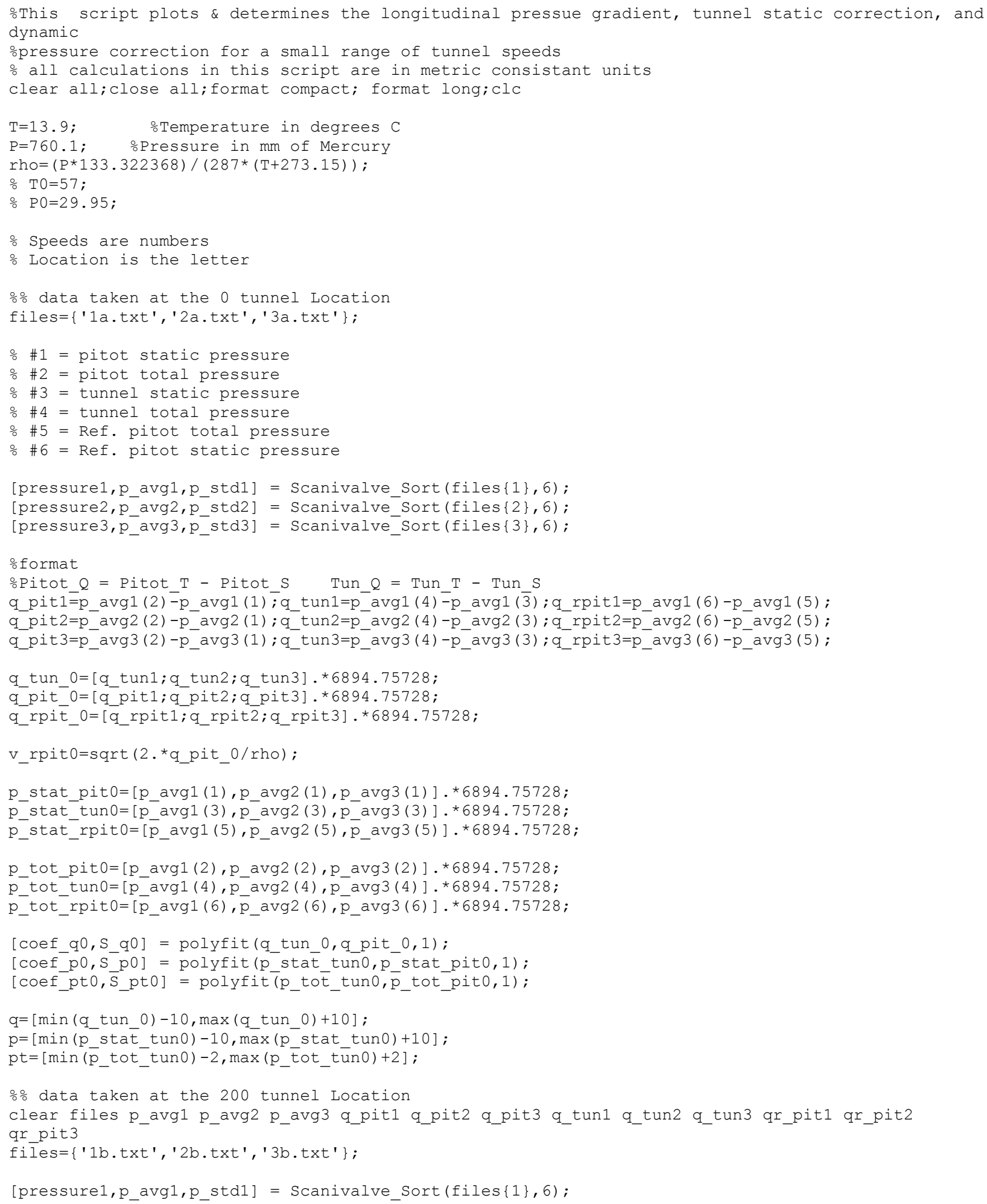




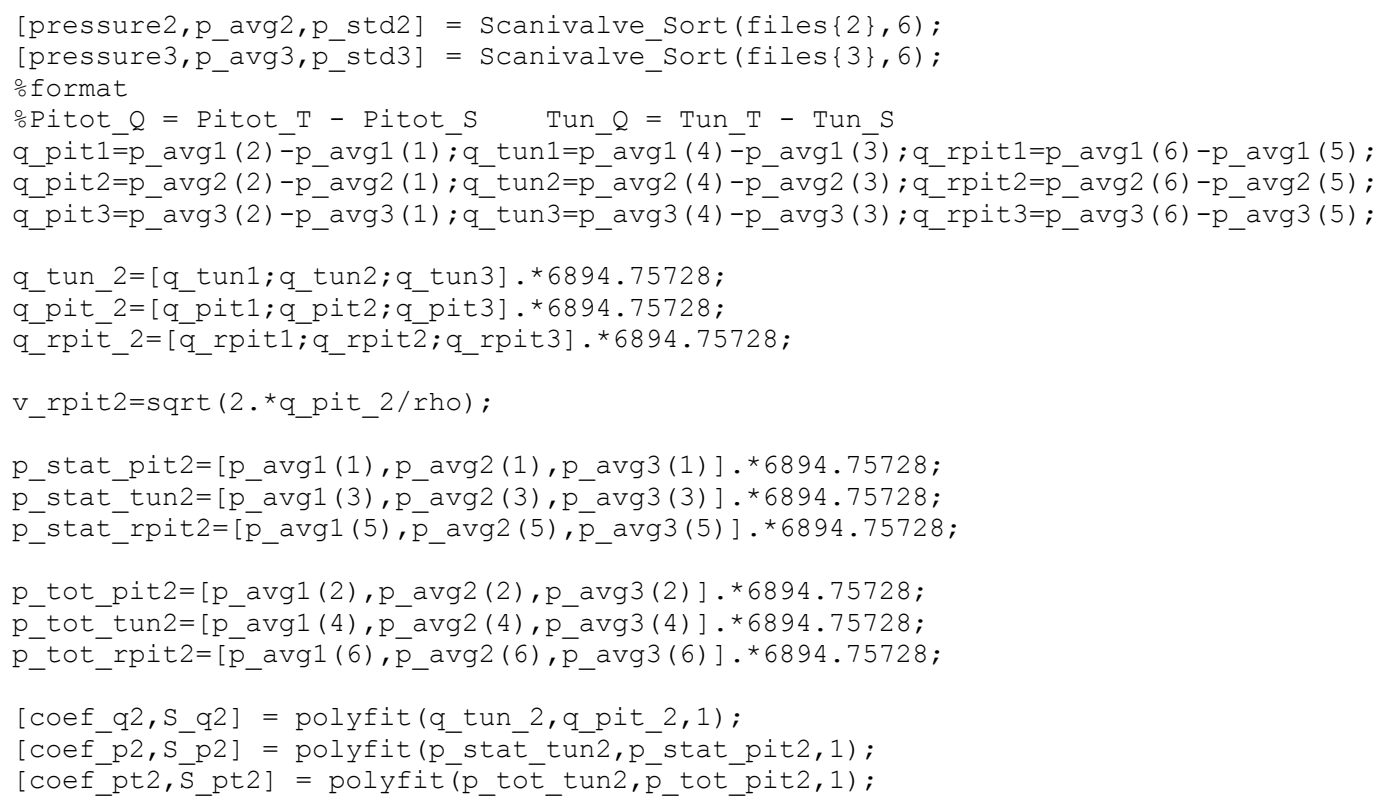




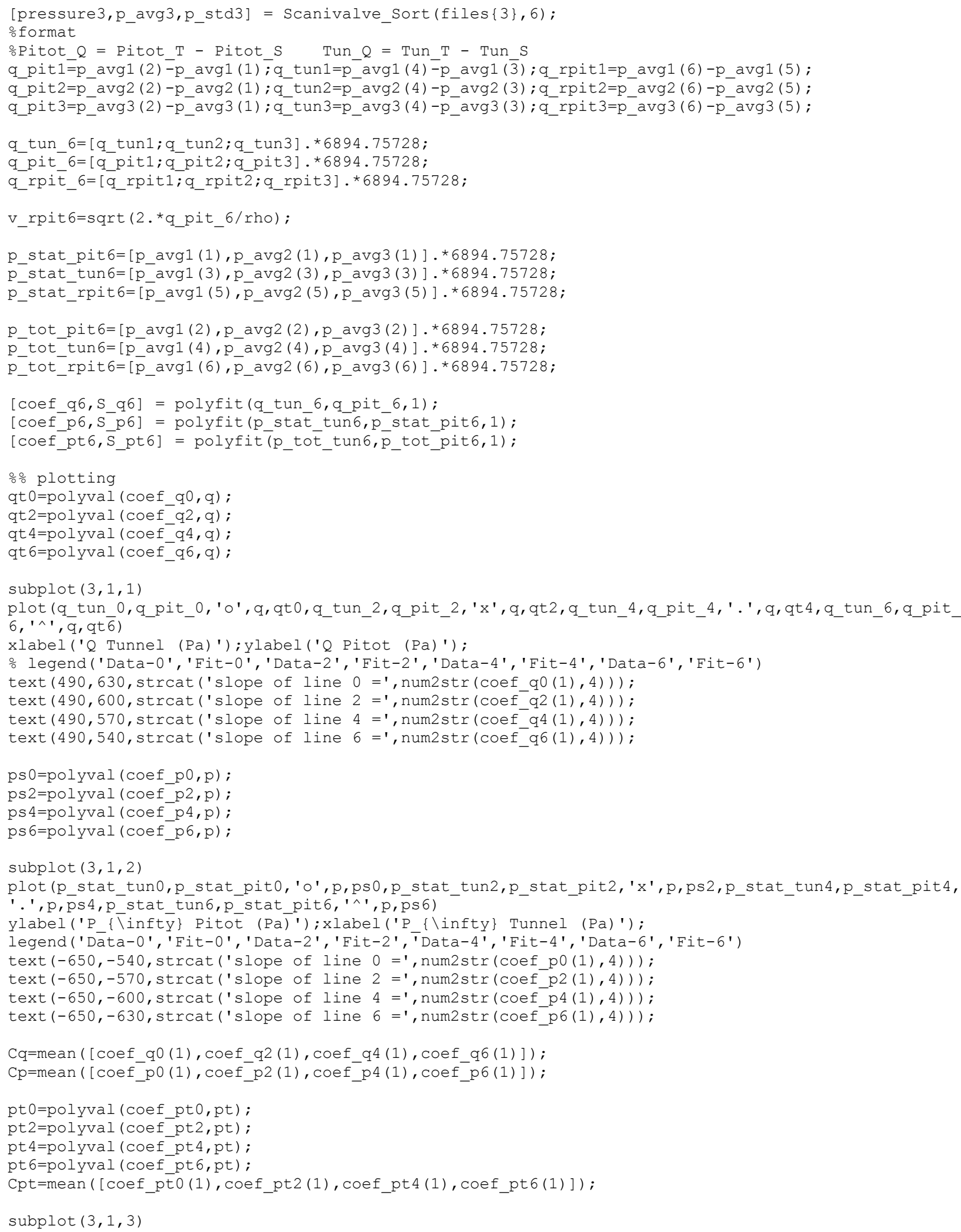




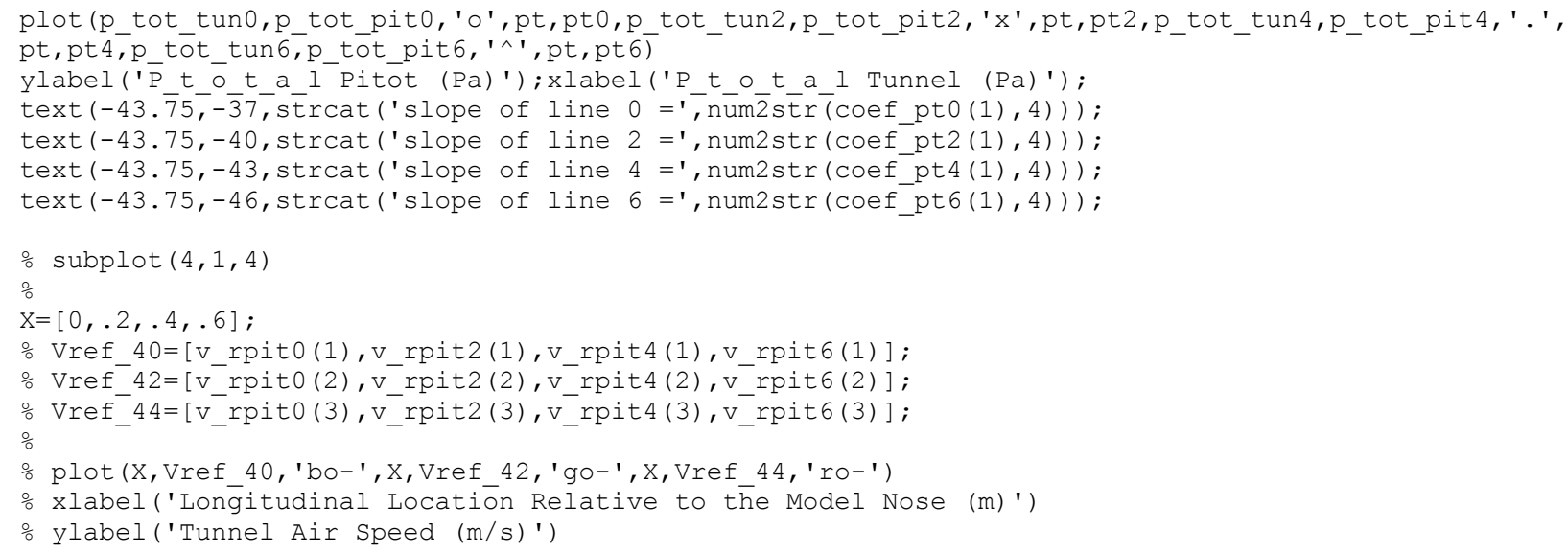




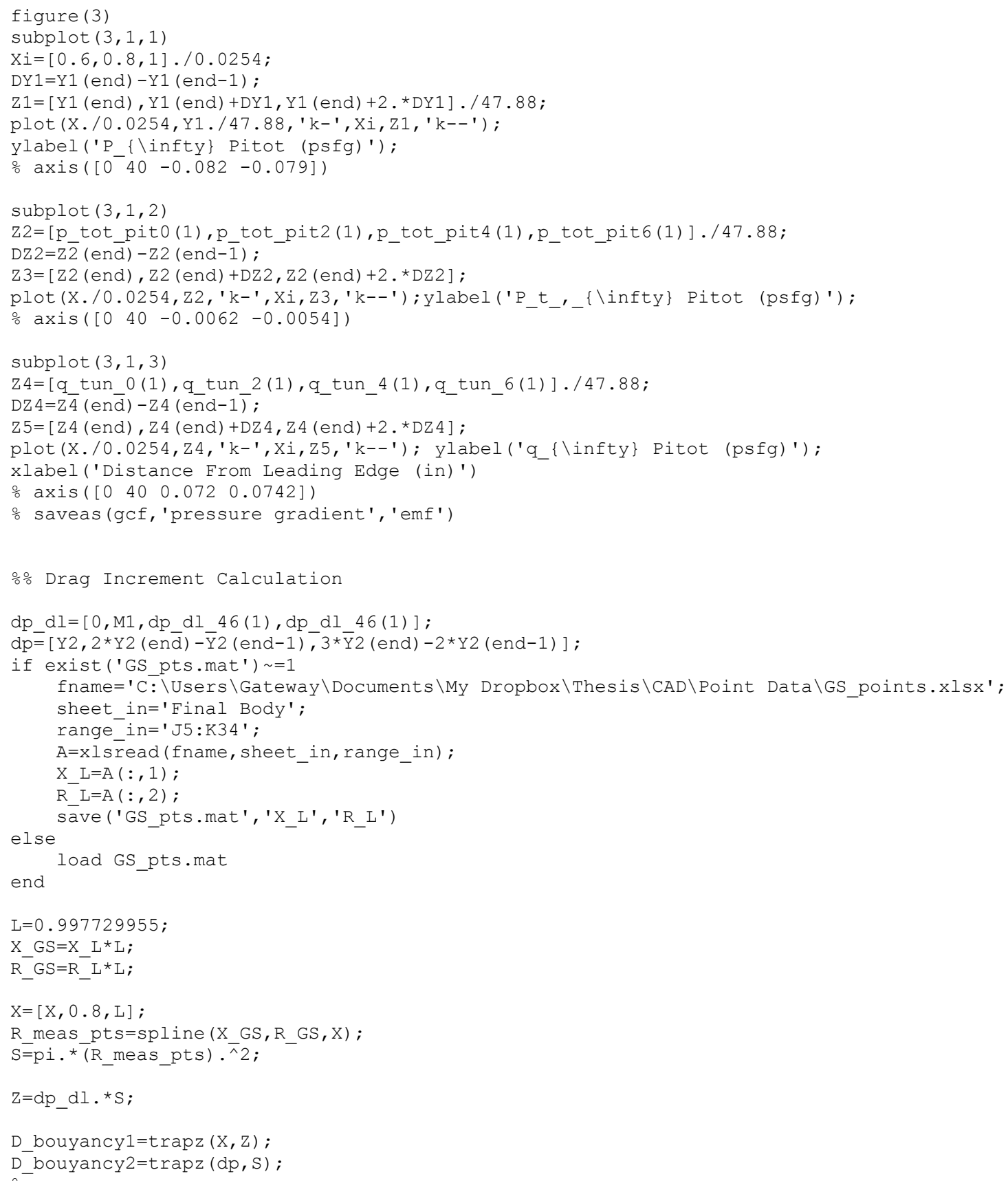




\section{APPENDIX D - DERIVATION OF THE AXIAL PRESSURE FORCE EQUATION}

Area of the outer part, not the flat base, of a cone

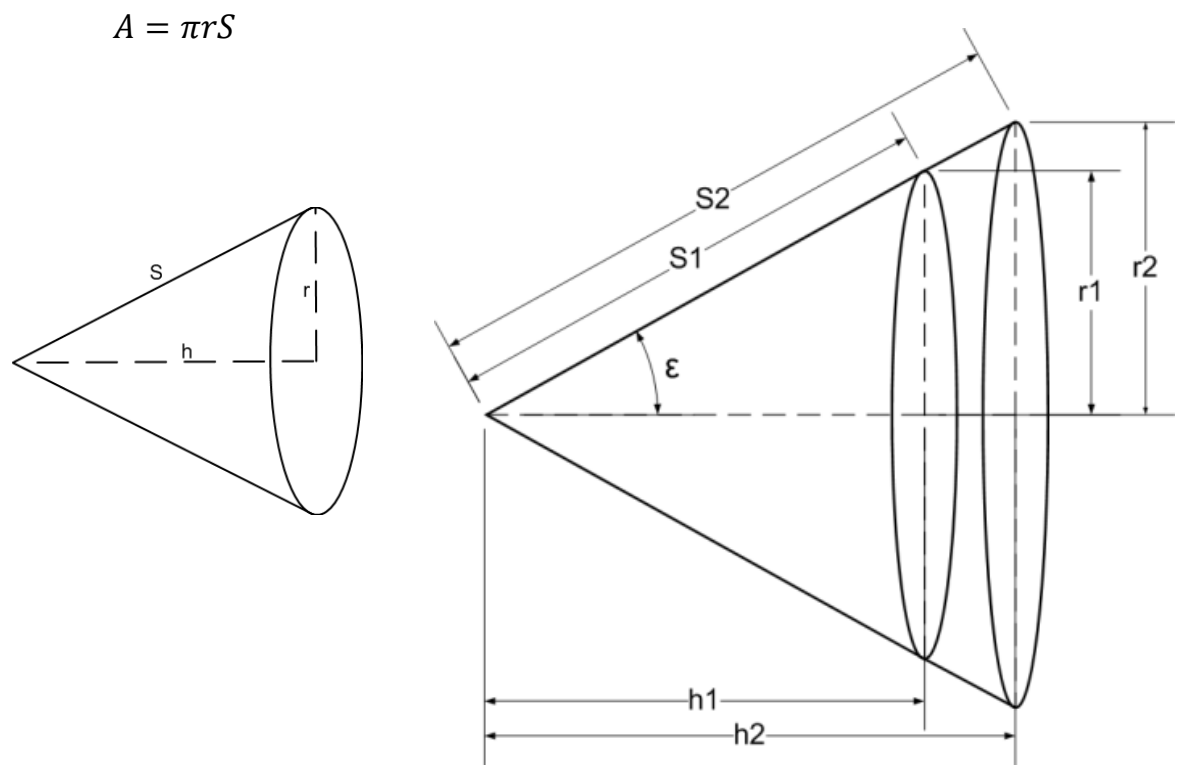

The frustum is what is left after cutting the top off by a plane that is parallel to the base.

$$
\begin{gathered}
A_{\text {frustum }}=A_{2}-A_{1} \\
A_{\text {frustum }}=\pi\left(r_{2} S_{2}-r_{1} S_{1}\right) \\
\Delta S=S_{2}-S_{1} \\
\Delta r=r_{2}-r_{1}
\end{gathered}
$$

Eliminate $\mathrm{r}_{1} \& \mathrm{~S}_{1}$

$$
\begin{gathered}
A_{\text {frustrum }}=A_{f}=\pi\left(r_{2} S_{2}-\left(r_{2}-\Delta r\right)\left(S_{2}-\Delta S\right)\right) \\
A_{f}=\pi\left(r_{2} S_{2}-\left(r_{2} S_{2}-r_{2} \Delta S-S_{2} \Delta r+\Delta r \Delta S\right)\right. \\
A_{f}=\pi\left(r_{2} S_{2}-r_{2} S_{2}+r_{2} \Delta S+S_{2} \Delta r-\Delta r \Delta S\right) \\
A_{f}=\pi\left(r_{2} \Delta S+S_{2} \Delta r-\Delta r \Delta S\right)
\end{gathered}
$$


Include only the component of the area in the axial direction

$$
\begin{gathered}
A_{f} \sin (\varepsilon)=\pi\left[r_{2} \Delta S \sin (\varepsilon)+S_{2} \Delta r \sin (\varepsilon)-\Delta r \Delta S \sin (\varepsilon)\right] \\
\sin (\varepsilon)=\frac{\Delta r}{\Delta S} \\
A_{f} \sin (\varepsilon)=\pi\left(r_{2} \Delta S \frac{\Delta r}{\Delta S}+S_{2} \Delta r \frac{\Delta r}{\Delta S}-\Delta r \Delta S \frac{\Delta r}{\Delta S}\right) \\
A_{f} \sin (\varepsilon)=\pi\left(r_{2} \Delta r+\Delta r^{2} \frac{S_{2}}{\Delta S}-\Delta r^{2}\right)
\end{gathered}
$$

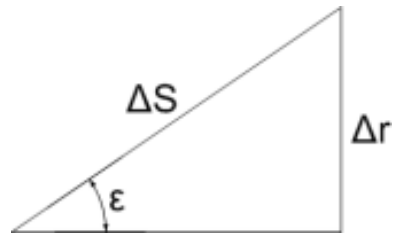

Eliminate $\mathrm{S}$, and $\Delta \mathrm{S}$ terms using similar triangles

$$
\begin{gathered}
\frac{\Delta S}{\Delta r}=\frac{S_{2}}{r_{2}} \\
A_{f} \sin (\varepsilon)=\pi\left(r_{2} \Delta r+\Delta r^{2} \frac{r_{2}}{\Delta r}-\Delta r^{2}\right) \\
A_{f} \sin (\varepsilon)=\pi\left(r_{2} \Delta r+r_{2} \Delta r-\Delta r^{2}\right) \\
A_{f} \sin (\varepsilon)=2 \pi r_{2} \Delta r-\pi \Delta r^{2}
\end{gathered}
$$

Net pressure force on an individual panel

$$
\Delta F_{a, p_{i}}=p_{i} * A_{i} * \sin \left(\varepsilon_{i}\right)
$$




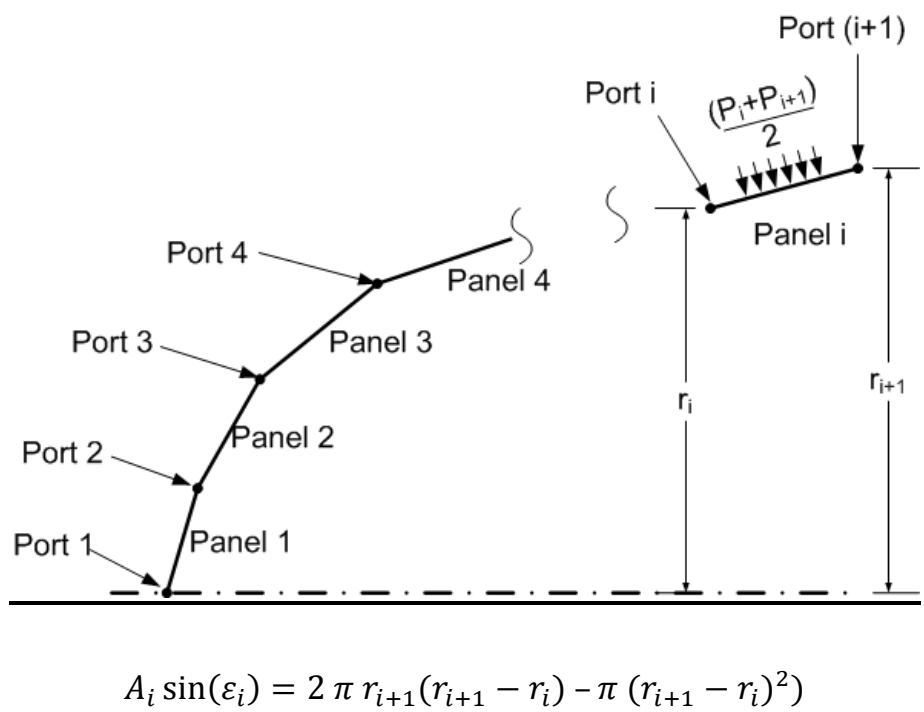

Pressure Force on all panels

A positive average pressure and a positive panel angle results in a force in the negative direction (drag). Therefore, a negative $F_{a, p}$ value is drag, and a positive $F_{a, p}$ value is thrust

$$
\begin{gathered}
F_{a, p}=-\sum_{i=1}^{N-1}\left(p_{i, a v e}-p_{\infty}\right) A_{i} \sin \left(\varepsilon_{i}\right) \\
\left.F_{a, p}=\sum_{i=1}^{N-1}-\left(p_{i, a v e}-p_{\infty}\right)\left[2 \pi r_{i+1}\left(r_{i+1}-r_{i}\right)-\pi\left(r_{i+1}-r_{i}\right)^{2}\right)\right] \\
\mathrm{N}=\text { number of ports }=\text { number of panels }+1 \\
p_{i, a v e}=\frac{\left(p_{i}+p_{i+1}\right)}{2} \\
F_{a, p}=\sum_{i=1}^{N-1}-\left[\frac{\left(p_{i}+p_{i+1}\right)}{2}-p_{\infty}\right]\left[\left(2 \pi r_{i+1}{ }^{2}-2 \pi r_{i+1} r_{i}\right)-\pi\left(r_{i+1}{ }^{2}-2 r_{i+1} r_{i}+r_{i}{ }^{2}\right)\right] \\
2
\end{gathered}
$$




$$
\begin{aligned}
& F_{a, p}=\sum_{i=1}^{N-1}-\left[\frac{\left(p_{i}+p_{i+1}\right)}{2}-p_{\infty}\right]\left[2 \pi r_{i+1}^{2}-2 \pi r_{i+1} r_{i}-\pi r_{i+1}{ }^{2}+2 \pi r_{i+1} r_{i}-\pi r_{i}{ }^{2}\right] \\
& F_{a, p}=\sum_{i=1}^{N-1}-\left[\frac{\left(p_{i}+p_{i+1}\right)}{2}-p_{\infty}\right]\left[\pi r_{i+1}^{2}-\pi r_{i}^{2}\right] \\
& F_{a, p}=\sum_{i=1}^{N-1}-\left[\frac{\left(p_{i}+p_{i+1}\right)}{2}-p_{\infty}\right] \pi\left(r_{i+1}^{2}-r_{i}^{2}\right) \\
& F_{a, p}=\sum_{i=1}^{N-1}-\left[\frac{\left(p_{i}+p_{i+1}-2 p_{\infty}\right)}{2}\right] \pi\left(r_{i+1}^{2}-r_{i}^{2}\right) \\
& F_{a, p}=\sum_{i=1}^{N-1}-\left[\frac{\left(p_{i}-p_{\infty}\right)+\left(p_{i+1}-p_{\infty}\right)}{2}\right] \pi\left(r_{i+1}{ }^{2}-r_{i}^{2}\right) \\
& C_{a, p}=\frac{F_{a, p}}{q_{\infty} A_{r e f}}=\sum_{i=1}^{N-1}-\frac{1}{2}\left[\frac{\left(p_{i}-p_{\infty}\right)+\left(p_{i+1}-p_{\infty}\right)}{q_{\infty}}\right] \frac{\pi\left(r_{i+1}{ }^{2}-r_{i}{ }^{2}\right)}{A_{\text {ref }}} \\
& C_{a, p}=\sum_{i=1}^{N-1}-\frac{1}{2}\left[\frac{\left(p_{i+1}-p_{\infty}\right)}{q_{\infty}}+\frac{\left(p_{i}-p_{\infty}\right)}{q_{\infty}}\right] \frac{\pi\left(r_{i+1}^{2}-r_{i}^{2}\right)}{A_{r e f}} \\
& C_{a, p}=\sum_{i=1}^{N-1}-\left[\frac{C_{p, i+1}+C_{p, i}}{2}\right] \frac{\pi\left(r_{i+1}{ }^{2}-r_{i}^{2}\right)}{A_{r e f}}
\end{aligned}
$$




\section{APPENDIX E - SURFACE POINTS AND PRESSURE PORT LOCATIONS}

Body Points used in Surface Spline in CAD

\begin{tabular}{|c|c|}
\hline \multicolumn{2}{|c|}{ Final Body } \\
\hline $\mathrm{X} / \mathrm{L}$ & $\mathrm{R} / \mathrm{L}$ \\
\hline 0 & 0 \\
\hline 0.02432 & 0.03511 \\
\hline 0.0514 & 0.0552 \\
\hline 0.06781 & 0.06523 \\
\hline 0.10632 & 0.085 \\
\hline 0.1517 & 0.10379 \\
\hline 0.20301 & 0.12109 \\
\hline 0.25922 & 0.13637 \\
\hline 0.31923 & 0.14921 \\
\hline 0.3503 & 0.15459 \\
\hline 0.41377 & 0.16307 \\
\hline 0.44587 & 0.16611 \\
\hline 0.50997 & 0.16966 \\
\hline 0.54165 & 0.17001 \\
\hline 0.60351 & 0.16775 \\
\hline 0.66222 & 0.161698 \\
\hline 0.68999 & 0.15733 \\
\hline 0.740909 & 0.146029 \\
\hline 0.769805 & 0.137048 \\
\hline 0.809416 & 0.118753 \\
\hline 0.829959 & 0.100209 \\
\hline Slot Gap \\
\hline 0.8449 & 0.0881 \\
\hline 0.8552 & 0.0768 \\
\hline 0.8693 & 0.0638 \\
\hline 0.8781 & 0.0575 \\
\hline 0.8869 & 0.0522 \\
\hline 0.8957 & 0.0469 \\
\hline 0.9133 & 0.0388 \\
\hline 0.9398 & 0.0297 \\
\hline 0.975 & 0.0238 \\
\hline & \\
\hline
\end{tabular}

For further details of surface, see CAD model 
Pressure Port Locations

Shaded port was removed from the analysis due to leak

\begin{tabular}{|c|c|c|c|}
\hline $\mathrm{x} / \mathrm{L}$ & $r / L$ & $\mathrm{X} / \mathrm{L}$ & $r / L$ \\
\hline 0 & 0 & 0.73955 & 0.14641 \\
\hline 0.005 & 0.0093367 & 0.75403 & 0.14215 \\
\hline 0.015 & 0.024471 & 0.76674 & 0.13807 \\
\hline 0.025366 & 0.036135 & 0.77791 & 0.13427 \\
\hline 0.036111 & 0.045158 & 0.78772 & 0.1305 \\
\hline 0.04725 & 0.052618 & 0.79633 & 0.12654 \\
\hline 0.058796 & 0.059785 & 0.8039 & 0.12235 \\
\hline 0.070765 & 0.066945 & 0.81054 & 0.11796 \\
\hline 0.083171 & 0.073734 & 0.81638 & 0.11346 \\
\hline 0.096032 & 0.080179 & 0.8215 & 0.10895 \\
\hline 0.10936 & 0.086381 & 0.826 & 0.10452 \\
\hline 0.12318 & 0.092423 & 0.854 & 0.083454 \\
\hline 0.13751 & 0.098309 & 0.858 & 0.079105 \\
\hline 0.15236 & 0.10404 & 0.86364 & 0.07333 \\
\hline 0.16775 & 0.1096 & 0.8716 & 0.066026 \\
\hline 0.18371 & 0.11501 & 0.88282 & 0.057682 \\
\hline 0.20025 & 0.12025 & 0.89864 & 0.048097 \\
\hline 0.21739 & 0.12533 & 0.92095 & 0.037759 \\
\hline 0.23516 & 0.13025 & 0.95242 & 0.027818 \\
\hline 0.25359 & 0.13499 & 0.9968 & 0.023037 \\
\hline 0.27268 & 0.13955 & & \\
\hline 0.29248 & 0.1439 & & \\
\hline 0.313 & 0.14803 & & \\
\hline 0.33427 & 0.15192 & & \\
\hline 0.35632 & 0.15554 & & \\
\hline 0.37918 & 0.15886 & & \\
\hline 0.40287 & 0.16185 & & \\
\hline 0.42743 & 0.16446 & & \\
\hline 0.45289 & 0.16667 & & \\
\hline 0.50664 & 0.16957 & & \\
\hline 0.56336 & 0.16967 & & \\
\hline 0.59929 & 0.16803 & & \\
\hline 0.63085 & 0.16544 & & \\
\hline 0.65857 & 0.1622 & & \\
\hline 0.68291 & 0.15854 & & \\
\hline 0.70429 & 0.15466 & & \\
\hline 0.72306 & 0.15061 & & \\
\hline
\end{tabular}


Appendix F - Scanivalve Information \& Troubleshooting

\section{APPENDIX F - SCANIVALVE INFORMATION \& TROUBLESHOOTING}

The Scanivalve system is a bit "touchy." It seems to give random errors and have random problems not related to any outside occurrence, or user input about once every few hours of operation. This could possibly be solved by contacting the manufacturer and sending in the unit for a calibration and software update. To date, these have been too expensive to complete.

The first fix when things out of the ordinary start to happen is to shut off the Scanivalve system with the switch on the power supply and close all the software programs, including RAD.exe, and restarting the hardware, then the software. This usually fixes most problems.

The final fix-all for the system is to overwrite the system settings/configuration file with backups of the old settings files. The system settings files are in the same folder that contains the RAD.exe file. It is usually in the "C:I Programs FilesIScanivalve" folder or similar. Inside the folder should be another folder named "backup files" or similar. Copy the contents of the backup folder into the main system folder and overwrite all files when asked.

One way to increase the reliability was to use different software from the traditional Radlink. This software is called ScanTEL. It is much more reliable and faster to use. There is a very good manual for this software so I will not elaborate on it here. The file is called "scantel_v101.pdf". It does not have all the buttons that RADLink does, but it has a simple command line interface and dropdown menus to do everything you need to do. This program produces '*.txt' ASCii files instead of the '*.dat' file that the RADLink software creates. That means that the normal Matlab analysis files will not work with the new data file. However, this is not insurmountable and a simple function that does the same as the old functions has been written and is a much smaller, simpler, and faster program.

If you are a graduate student and need to use the scanivalve, then I suggest that you READ THE MANUALS. You may wrongly think that you do not have time to read the manuals, but what you really don't have time for is the time wasted trying to figure out what is going on instead of just reading the manuals. 


\section{APPENDIX G - PRESSURE MEASUREMENT FITTINGS, TUBING \& GENERAL ADVICE}

- $\quad$ Proper fittings are essential to a leak proof tubing system. Even small leaks will make your data useless.

- Hose is flexible soft rubber/silicone/etc. Tube/tubing is metal. Use the correct terms when describing the system

- The hose that is used throughout the wind tunnel is $1 / 16 "$ ID tubing. The tubing is nominally $1 / 16$ " OD.

- Pressure readings are unreliable, unbelievable, and don't have any integrity unless the entire pressure tubing system is thoroughly leak checked beforehand and proven to be free from leaks.

- Do not use fittings that are the wrong size.

- Do not use tubing that is the wrong size.

- $\quad$ Do not use hose that is the wrong size.

- Do not join tubing by inserting one size hose into or around another hose. Always use the correct hard fitting. If you do not have the correct fitting, then go and buy one. McMaster Carr has a huge selection of fittings, and in an emergency, local auto parts stores have a wide selection of plastic fittings, but they generally only stock low quantities.

- Do not use silicone sealants, RTV, or any glue on a joint to seal it. Bathroom/kitchen caulk has no place in a wind tunnel. You will see it used in some places, but do not repeat their mistakes. It did not work to seal the wind tunnel sections together and caused more leaks and extra work than it helped.

- Do not use tape on a joint to seal it, especially duct tape or electrical tape. These leave a sticky residue that is very difficult to remove later on. Tape is very difficult to get to lay flat enough to seal air tight.

- Get the softest hose you can find. The softer the hose, the easier it is to seal and stretch around a hard line/tube, as well as remove from a hard line/tube.

- If going from a soft line (hose) to a hard line, the hard line should have barbs, or a bulge that you need to push the tubing over in order to seal the tube a against the hard line. If budget does not permit, then buy the hard line that is at least 0.010 " large OD than the ID of the soft line and use it instead. McMaster Carr has a 
large selection of stainless tubing in many different sizes to work with whatever size you hose you have. They also have many different sizes of hose to choose from.

- Use as few fittings and junctions as possible, less possibility for leaks and less items that need to be leak checked.

- Have respect for all current equipment and systems in the wind tunnel. Many smart people have come before you, and they have set things up and configured them in a certain way for a reason. Do not be quick to change things and always get approval for any changes from the Aero Lab Technician (Cody Thompson) and Dr. Tso prior to making the change, no matter how small you think they are.

- Any change to any system in the tunnel should be non-permanent and should be undone after use. Even though the changes are non-permanent, the quality of the workmanship and materials of the change should be as if it is permanent. Generally speaking, anytime you try to cut corners, you and someone else after you will pay the price.

- Do things the right way, and you will cut down on the number of problems you experience with your experiment. It will also gain you, and your project, credibility.

- $\quad$ Take some time, look at some of the models in the wind tunnel room, and examine how they were built. Figure out why they were made the way they were. Figure out which models are quality models that produced good data, and which ones produced bad data. Incorporate the features of the good models into your model and avoid the pitfalls of the bad models.

- Remember, bad data always has a source. Only after you have eliminated ALL the possible sources of error can you trust your data. 
Appendix H - Cal Poly Wind Tunnel VFD Operation

\section{APPENDIX H - CAL POLY WIND TUNNEL VFD OPERATION}

\section{Purpose:}

This describes the current and previous operation of the Variable Frequency Drive (VFD) for the Cal Poly Aero Wind Tunnel.

\section{Previous Method of Operation:}

The electric motor that drives the fan for the wind tunnel is controlled by the VFD. The VFD's input was the voltage from a potentiometer mounted on the front panel. This potentiometer is a single turn version, and thus required very little movement of the knob to affect the wind tunnel speed significantly. This sensitivity resulted in the speed setting of the wind tunnel to not be very repeatable. In addition, The VFD output frequency always varied by $\pm 0.3 \mathrm{~Hz}$ during wind tunnel operation. It was thought that electrical noise in the circuit of the potentiometer could be causing these oscillations. This was disproved by observing the potentiometer voltage output during operation of the wind tunnel. The voltage signal from the potentiometer was steady and did not vary appreciably.

\section{New Method of Operation:}

The VFD can now take inputs directly from the keypad on the front panel. When the VFD is fist powered up with the large lever on the upper right of the front panel, the operator needs to evaluate what is displayed on the front panel. At the bottom of the screen, one of two scenarios will be displayed. The first scenario is if there are options displayed on the screen above all 3 of the 'function' (F1, F2, F3) buttons. This means that the VFD control is in what is called 'Keypad Mode' this is when the VFD takes commands from the keypad only. The other scenario is if there is only an option displayed above the F2 function button. This means that the VFD is in "Terminal Mode". In Terminal Mode, the VFD takes some commands from the keypad and some commands from the buttons and potentiometer on the front panel. These buttons and potentiometer are connected to the VFD through a terminal strip, hence the name.

If the VFD is in Terminal Mode, then press the F2 button to put the VFD in Keypad Mode. Above the F2 button on the screen should appear ' $\mathrm{T} / \mathrm{K}$ '. If this is not displayed, then please refer to the manual VD0C06S305C to 
determine which menu you are in, and how to get to the correct menu. (If the keypad screen is just displaying a lot of information about the VFD and motor, then try to press 'ENT' to try to get to the correct menu)

\section{Directly Enter the Desired Frequency}

Once in Keypad Mode, press the button for a decimal point. This will bring up a popup window to enter the desired frequency. Enter the desired frequency and press 'ENT (enter). When you are ready to start the wind tunnel, press the 'RUN' button. The VFD will ramp up at a rate of $2 \mathrm{~Hz}$ per second. So to get to $40 \mathrm{~Hz}$, it will take approximately 20 seconds. Once running, the procedure can be repeated to change the tunnel speed, but the change is implemented instantaneously when you press ENT after entering the new frequency.

\section{Scroll to the Desired Frequency:}

Once in Keypad Mode, you can either enter in an initial guess from the previous method, or press the RUN button first. Once the RUN button is pressed, the VFD is 'running', even if the commanded frequency is $0 \mathrm{~Hz}$. From there you can change the commanded frequency by using the up and down arrows. Pressing the arrows slowly will result in the command frequency changing by $0.1 \mathrm{~Hz}$. Pressing the arrows repeatedly (faster) will change the frequency by a larger increment that depends on how fast you pressed the arrows. Jumps of 10-20 Hz are common if you press the buttons fast enough. (Even though the commanded frequency has jumped quickly, the output frequency to the motor will never change by more than $2 \mathrm{~Hz}$ per second)

\section{Stopping the Tunnel:}

Press the STOP button either on the keypad or on the upper control panel to stop the tunnel. It will slow down at a rate of $2 \mathrm{~Hz}$ per second, and eventually come to a stop. Once stopped, wait at least 5 minutes for the VFD to cool down before switching the unit completely off with the big lever on the upper right of the front panel.

\section{Sample Conversion:}




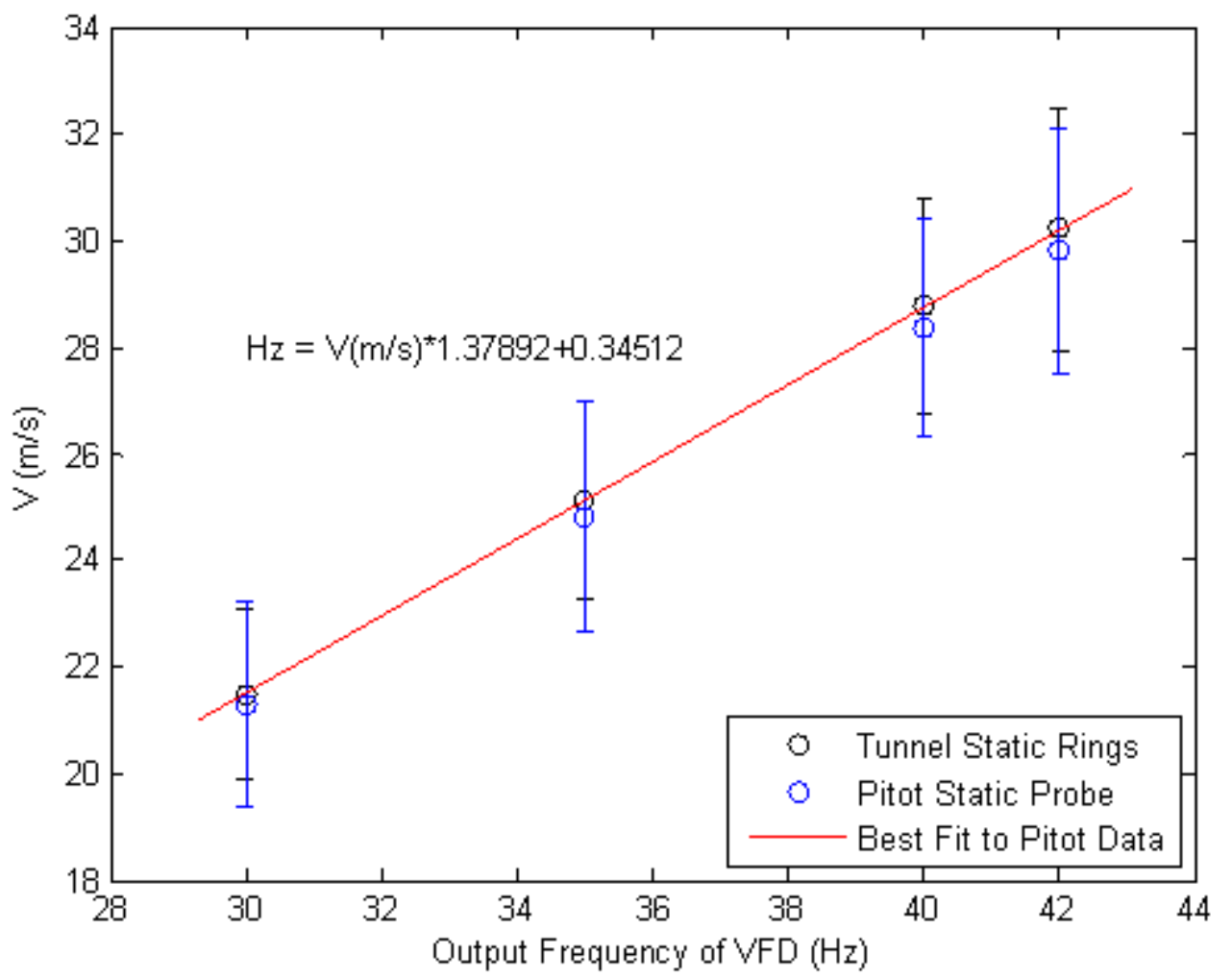

